\title{
Impact of G12 Mutations on the Structure of K-Ras Probed by Ultraviolet Photodissociation Mass Spectrometry
}

Michael B. Cammarata ${ }^{1}$, Christopher L. Schardon ${ }^{2}$, M. Rachel Mehaffey ${ }^{1}$, Jake Rosenberg ${ }^{1}$, Jonathan Singleton ${ }^{1}$, Walter Fast ${ }^{3}$, Jennifer S. Brodbelt ${ }^{* 1}$

${ }^{1}$ Department of Chemistry

${ }^{2}$ Graduate Program in Biochemistry

${ }^{3}$ Division of Chemical Biology and Medicinal Chemistry, College of Pharmacy

University of Texas

Austin, TX 78712

\section{Supporting Information:}

Supporting information contains Figures S1-12 plus a number of tables (Tables S1-S9). The figures include color-coded diagrams of the crystal structure of K-Ras to facilitate identification of structural elements, ESI and UVPD mass spectra of the K-Ras complexes, a graph showing the average mass shift associated with complexation of the GDP or GDPnP ligands, expanded regions of the UVPD fragmentation trends of the complexes, several graphical displays of statistical treatments used to assess the UVPD data, and a graphical display illustrating the establishment of significance for the variations in backbone cleavage efficiencies. The tables summarize all product ions identified upon UVPD of the various K-Ras complexes (Tables S1-S8) and a summary of residues for which UVPD is enhanced or suppress for each complex (Table SO). 
Protein Expression and Purification: Recombinant human wild-type K-Ras (isoform 2, residues 1-169 $(18,954 \mathrm{Da})$ was heterologous expressed from previously described expression plasmids ${ }^{51}$ that also encode an $\mathrm{N}$-terminal hexahistidine affinity tag followed by a tobacco etch virus (TEV) protease cleavage site preceding the rest of the sequence. Expression and purification for the G12C, G12S or G12V variants was the same. Briefly, the expression vector was transformed into Escherichia coli BL21 (DE3) and subsequent cultures were grown in Terrific Broth containing kanamycin (50 mg/L) with shaking (250 RPM) at $37{ }^{\circ} \mathrm{C}$ until the optical density ${ }_{600 \mathrm{~nm}}$ reached $0.4-0.6$. Expression was induced through addition of isopropyl- $\beta$-D-thiogalactoside (IPTG, $0.5 \mathrm{mM}$ )) and incubation was continued overnight (approximately 18 h) at $18^{\circ} \mathrm{C}$. Cells were pelleted by centrifugation at $11,325 \times \mathrm{g}$ for $20 \mathrm{~min}$ at $4{ }^{\circ} \mathrm{C}$. The pellet was harvested and either used immediately for purification, or flash frozen in liquid nitrogen and stored at $-80^{\circ} \mathrm{C}$ for later use. A fresh or thawed pellet was resuspended in $100 \mathrm{~mL}$ Lysis Buffer ( $20 \mathrm{mM}$ Tris, $500 \mathrm{mM} \mathrm{NaCl}, 5$ $\mathrm{mM}$ imidazole at $\mathrm{pH}$ 8) supplemented with protease inhibitor cocktail (Roche complete EDTA free). $\beta$ Mercaptoethanol ( $2 \mathrm{mM}$ final concentration) was added to the solution and cells were lysed by sonication. Cell debris was pelleted by centrifugation at $39,191 \times g$ for 20 min at $4{ }^{\circ} \mathrm{C}$ and discarded. The remaining supernatant was incubated for $1 \mathrm{~h}$ with Qiagen Ni-NTA resin slurry $(6 \mathrm{~mL})$ pre-equilibrated in Lysis Buffer. The slurry was then transferred to a column and washed with Lysis Buffer $(50 \mathrm{~mL}$ ) supplemented with imidazole (20 mM). Hexahistidine-tagged K-Ras was then eluted using Lysis Buffer $(10 \mathrm{~mL})$ supplemented with imidazole $(200 \mathrm{mM})$. The hexahistidine affinity tag was removed by treatment with $N$-terminal Histagged TEV protease ( $1 \mathrm{mg}$ protease for every $25 \mathrm{mg}$ of total protein) along with supplementation of GDP (1 mg GDP for every $20 \mathrm{mg}$ of total protein) and dialyzing overnight against Dialysis Buffer (20 mM Tris, $300 \mathrm{mM} \mathrm{NaCl}, 5 \mathrm{mM}$ imidazole, $1 \mathrm{mM}$ dithiothreitol (DTT) and $0.5 \mathrm{mM}$ EDTA at pH 8). Following dialysis, the protein solution was diluted five-fold with Low-Salt Buffer (20 mM Tris, $50 \mathrm{mM} \mathrm{NaCl}$ at pH 8) and supplemented with $\mathrm{MgCl}_{2}(5 \mathrm{mM})$ and GDP (1 mM). Uncleaved hexahistidine-tagged K-Ras and $N$-terminal His-tagged TEV protease were removed from the final solution by incubation ( $1 \mathrm{~h}$ ) with Ni-NTA resin slurry 
$(3 \mathrm{~mL})$, pre-equilibrated with Low-Salt Buffer, followed by centrifugation to pellet and discard the Ni-NTA resin.

Each of the WT, G12C, G12S or G12V K-Ras variants was further purified by ion exchange chromatography using a Q-Sepharose Fast Flow column (8 mL, GE Healthcare Life Sciences). The column was washed using wash buffer ( $20 \mathrm{mM}$ Tris, $100 \mathrm{mM} \mathrm{NaCl}$ at pH 8). K-Ras protein was eluted using Elution Buffer (20 mM Tris, $250 \mathrm{mM} \mathrm{NaCl}$ at pH 8). Remaining high molecular weight impurities were removed using an Ultracel $50 \mathrm{kDa}$ molecular weight cutoff (MWCO) filter (Amicon Ultra 4 Centrifugal Filter). The filter flow-through containing K-Ras was exchanged into Storage Buffer (10 mM Tris, $25 \mathrm{mM} \mathrm{NaCl}$ at pH 8) using an Ultracel $10 \mathrm{kDa}$ MWCO filter (Amicon Ultra 4 Centrifugal Filter) and concentrated to a final concentration of $1-2 \mathrm{mg} / \mathrm{mL}$. Typical yields for each of the purified K-Ras variants were $5-10 \mathrm{mg} / 1 \mathrm{~L}$ culture.

K-Ras Nucleotide Exchange: K-Ras variants $(30 \mu \mathrm{M})$ were incubated in Exchange Buffer (25 mM Tris, , 10 mM EDTA, 1.5 mM guanosine 5'-[ $\beta, \gamma$-imido]triphosphate (GDPnP, Sigma Aldrich), $1 \mathrm{mM}$ dithiothreitol (DTT) at $\mathrm{pH}$ 8) for $1 \mathrm{~h}$. $\mathrm{MgCl}_{2}$ was subsequently added to the incubation to a final concentration of $25 \mathrm{mM}$, and the mixture was incubated for an additional $30 \mathrm{~min}$. The protein was then exchanged again into Exchange Buffer using an Ultracel $10 \mathrm{kDa}$ MWCO centrifugal filter (Amicon Ultra) and the above procedure was repeated a second time. In a final step, the protein was exchanged into a similar buffer that lacked EDTA ( $25 \mathrm{mM}$ Tris at $\mathrm{pH}$ 8). This procedure loads K-Ras with the nonhydrolyzable GTP mimic, GDPnP and results in approximately 95\% GDPnP loading as determined by MS (see below). 
Figure S1. Structures of (a) GDP, (b) GTP, and (c) stable GTP analog GDPnP. Sequence of K-Ras; residues are numbered from the initiator methionine (M) in subsequent figures.

a)<smiles>Nc1nc2c(ncn2C2OC(COP(=O)([O-])OP(=O)([O-])O)C(O)C2O)c(=O)[nH]1</smiles>

b)<smiles>Nc1nc2c(ncn2C2COC(COP(=O)([O-])OP(=O)([O-])OP(=O)([O-])O)C2O)c(=O)[nH]1</smiles><smiles></smiles>

d)

GMTEYKLVVVGAGGVGKSALTIQLIQNHFVDEYDPTIEDSYRKQVVIDGETCLLDILDTAGQEEYSAMRD QYMRTGEGFLCVFAINNTKSFEDIHHYREQIKRVKDSEDVPMVLVGNKCDLPSRTVDTKQAQDLARSYGI PFIETSAKTRQGVDDAFYTLVREIRKHKEK 
Figure S2. Histogram illustrating determination of significant changes in backbone cleavage efficiencies for all calculated changes in fragment ion abundances from the UVPD data (based on total ion current (TIC)). Blue bars show the frequency of bin level of the change in UVPD backbone cleavage efficiency (considering over 600 backbone cleavage efficiency values based on aggregation of all four proteins), and the red bars show the percent of the total population of backbone cleavages that correspond to the indicated changes. The green bars indicate the total additive percentage from small to large changes, thus illustrating that only the top 15\% of changes in UVPD backbone cleavage efficiency are considered significant. Any change greater than an absolute signal change of $0.0001 \%$ (generally at least a factor of $2 \mathrm{X}$ or greater change in abundance) falls within the top 15 percent of all variations in backbone cleavages and is considered a significant change.

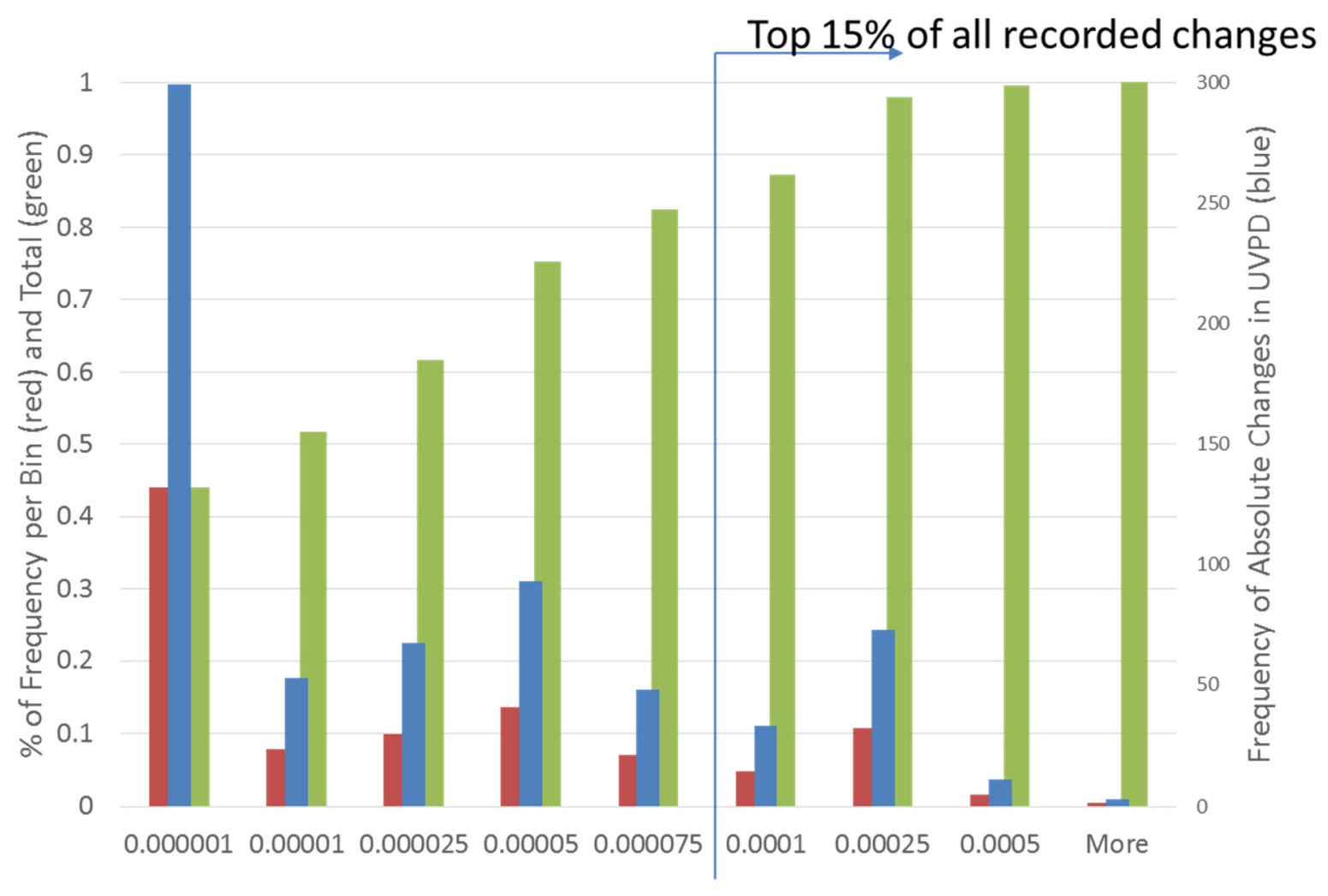

$\triangle T I C$ Abundance Bins \% 
Figure S3. Comparison of abundances of UVPD fragment ions for WT K-Ras versus each mutant $\mathrm{K}$-Ras for the $18+$ charge state (denatured protein). Analogous to the method used to evaluate backbone cleavage propensities for the native-like proteins, fragment ions arising from cleavage of the backbone positions between pairs of adjacent amino acids in the protein sequence were collectively summed. For example, all $\mathrm{N}$-terminal product ions ( $a_{n}, b_{n}$, and $c_{n}$ ions) arising from backbone cleavages that occur $\mathrm{C}$-terminal to a specific amino acid were summed with all the $\mathrm{C}$ terminal product ions ( $x_{R-n+1}, y_{R-n+1}$, and $z_{R-n+1}$ ions) arising from cleavages that occur $\mathrm{N}$-terminal to the same amino acid, where $\mathrm{n}$ is the residue number and $\mathrm{R}$ is the total number of amino acids in the protein. This value was calculated for each amino acid to convey the backbone cleavage efficiency adjacent to each particular residue. The values for the $12^{\text {th }}$ residue were not included because this residue varies for each K-Ras protein. This means that each plot is comprised of 169 comparative values (condensed from several hundred $a, b, c, x, y, z$ fragment ions), including three outliers (Y32, T128, F142) shown as red square symbols. The outliers are the same for all three plots because they arise from unusually high abundances of particular fragment ions that occur adjacent to Y32, T128, or F142 for the WT protein. The abundances of the fragmentation at each residue were plotted in the graphs for WT K-Ras relative to (a) G12V, (b) G12C, and (c) G12S. The correlation in the abundances and residues for the proteins is very good, aside from the three outliers.

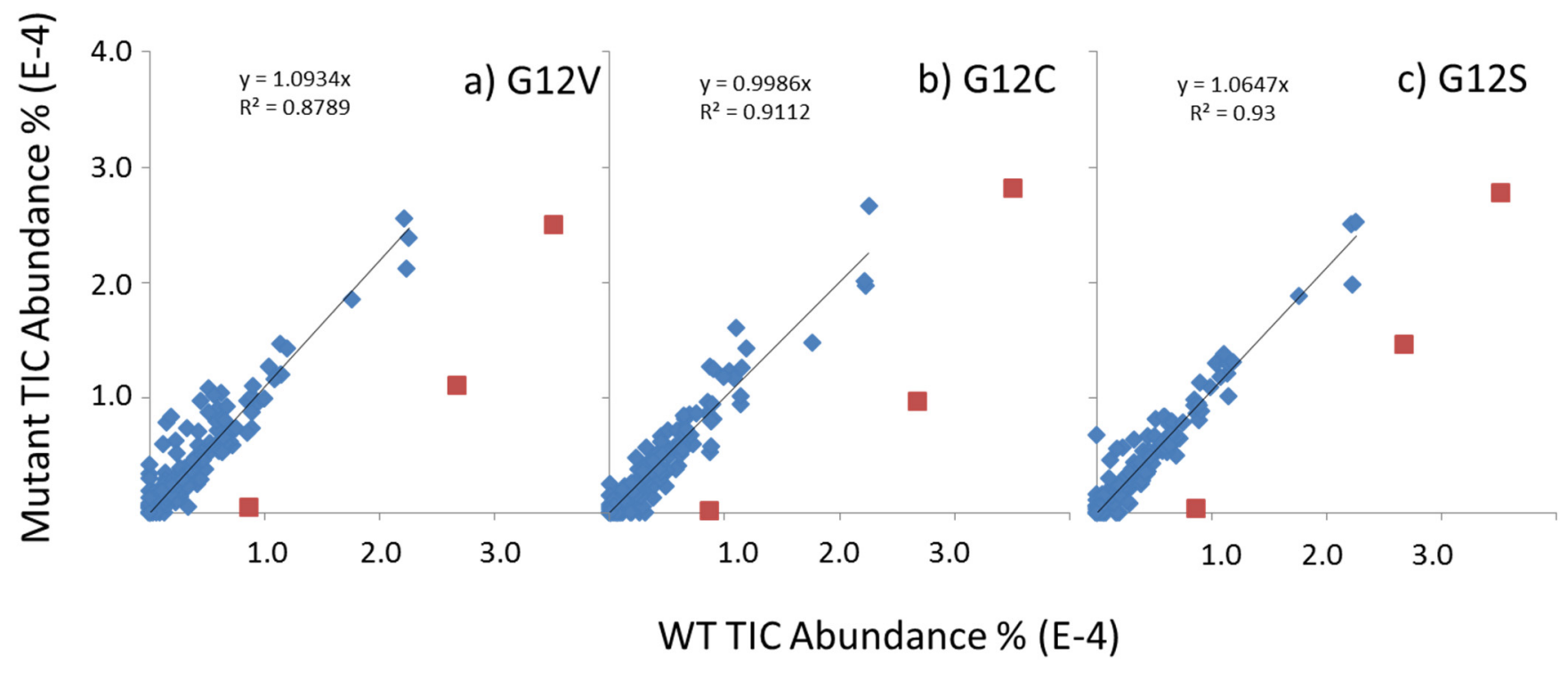


Figure S4. For each UVPD cleavage abundance at each position, the values obtained for the GDP complex and GDPnP complexes were subtracted (difference value), and then this difference value for each variant (G12C, G12V, G12S) was subtracted from the difference value for the WT protein. (a) The $t_{\text {calculated }}$ for each comparison at each residue is shown with confidence interval thresholds ( $t_{\text {critical }}$ ) between 90 and $99 \%$. (b) Histogram of $t_{\text {calculated }}$ over the whole data set with cumulative percentage in red dots. This statistical analysis correlates with the data shown in Figure 4.
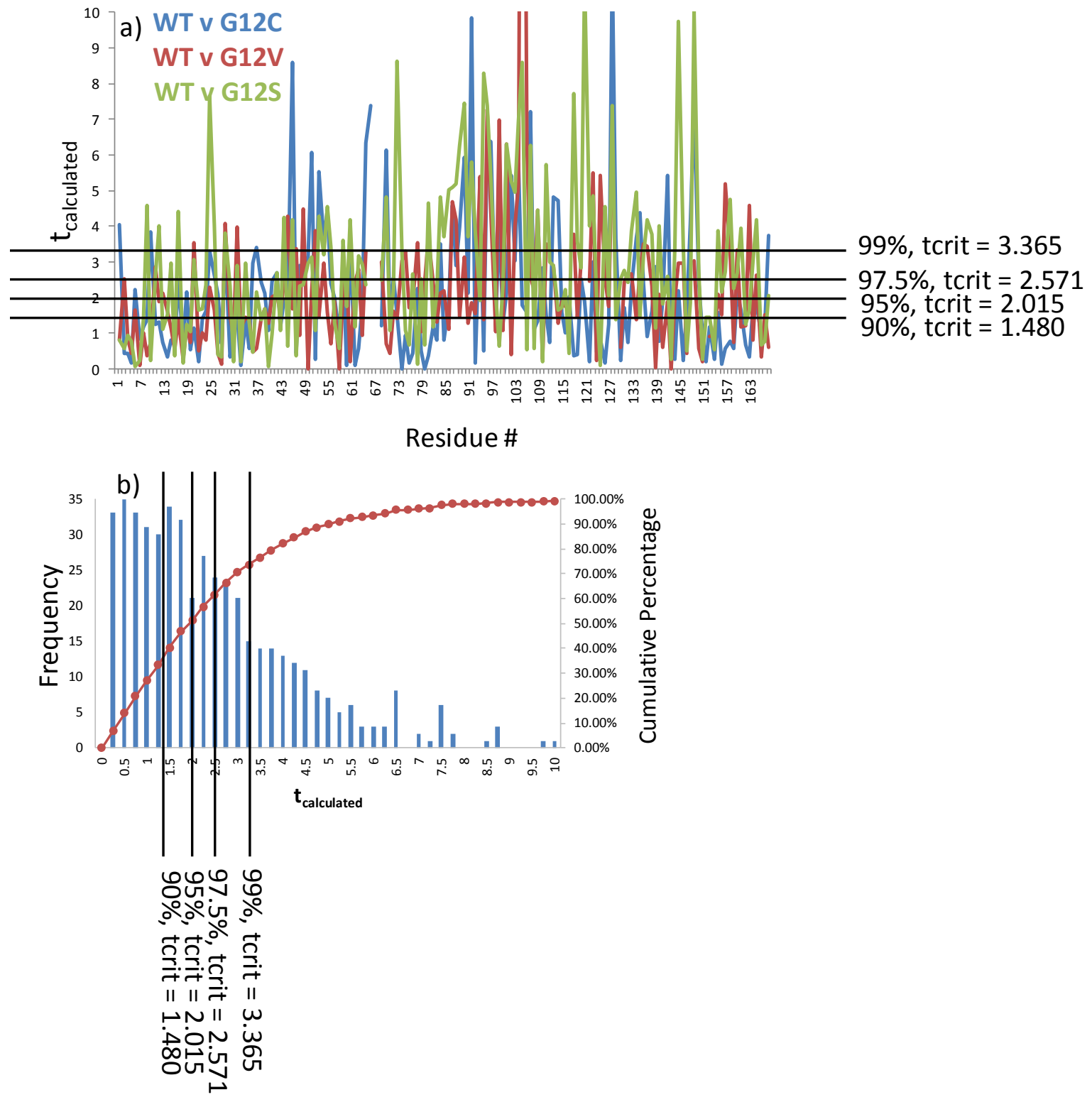
Figure S5. Color coded diagram for WT K-Ras showing various (a) helices, (b) loops and switches, and (c) beta strands based on the crystal structure 3GFT. The space-filled features represent GDPnP and $\mathrm{Mg}^{2+}$. Front and back views are shown on the left and right. Note that only switch I, switch II, and loop P are formally named in the crystal structure. The other helices, loops, switches, and beta strands are labelled here to facilitate discussion of the structure. Multicolored spheres represent the GDP•Mg ${ }^{2+}$, as follows: blue = nitrogen, green = carbon, red $=$ oxygen, white $=$ hydrogen, orange $=$ phosphorous.

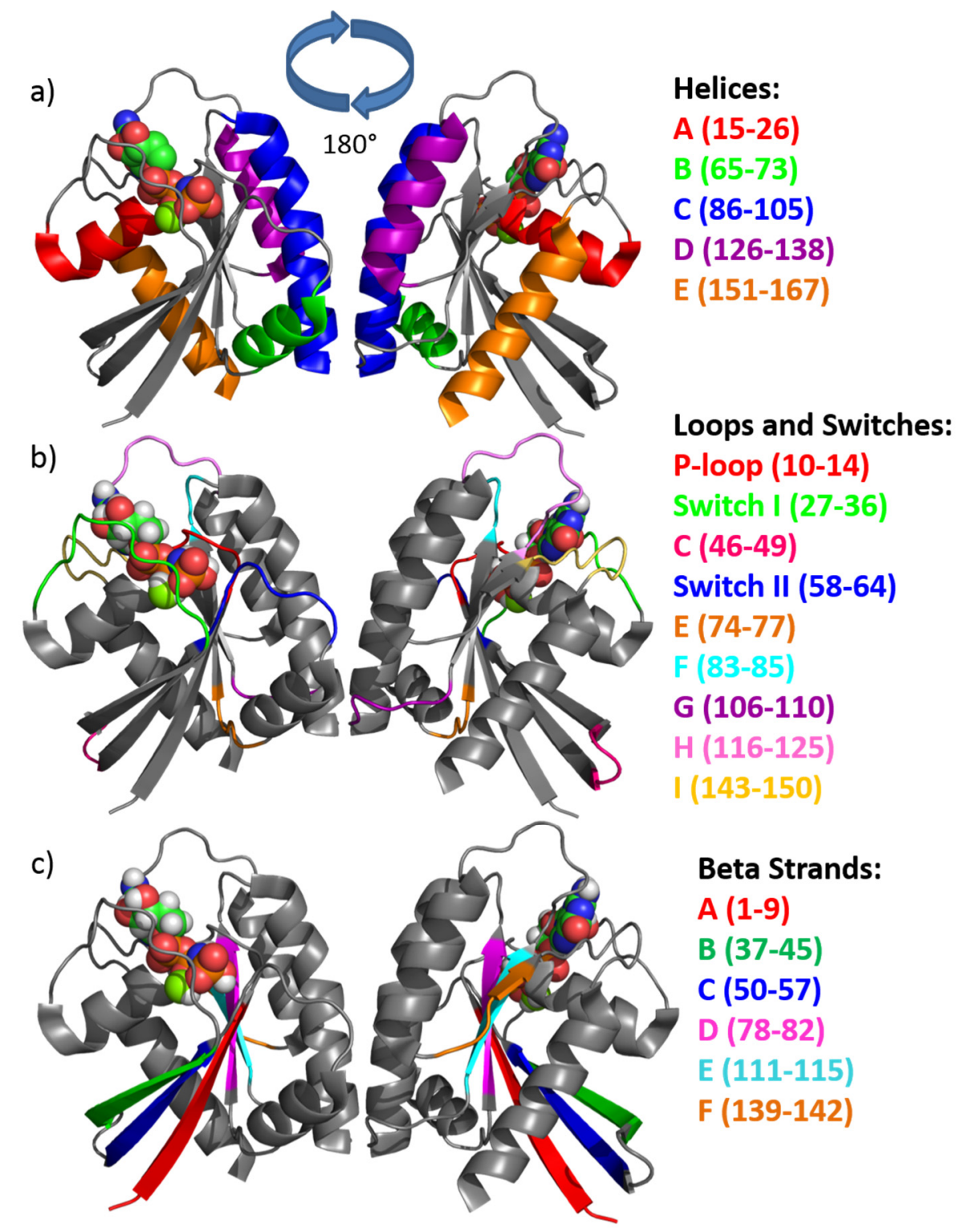


Figure S6. a) ESI mass spectrum of K-Ras (WT) sprayed from a $50 \mathrm{mM}$ ammonium acetate solution ( $\mathrm{pH} 7.8$ ) and expanded regions covering the $8+$ charge state for $\mathrm{b}$ ) WT•GDP•Mg$\left.{ }^{2+}, c\right)$

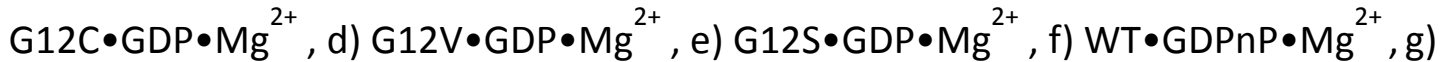

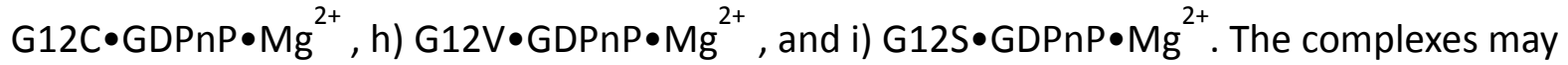
contain numerous additional sodium, magnesium and ammonium adducts.

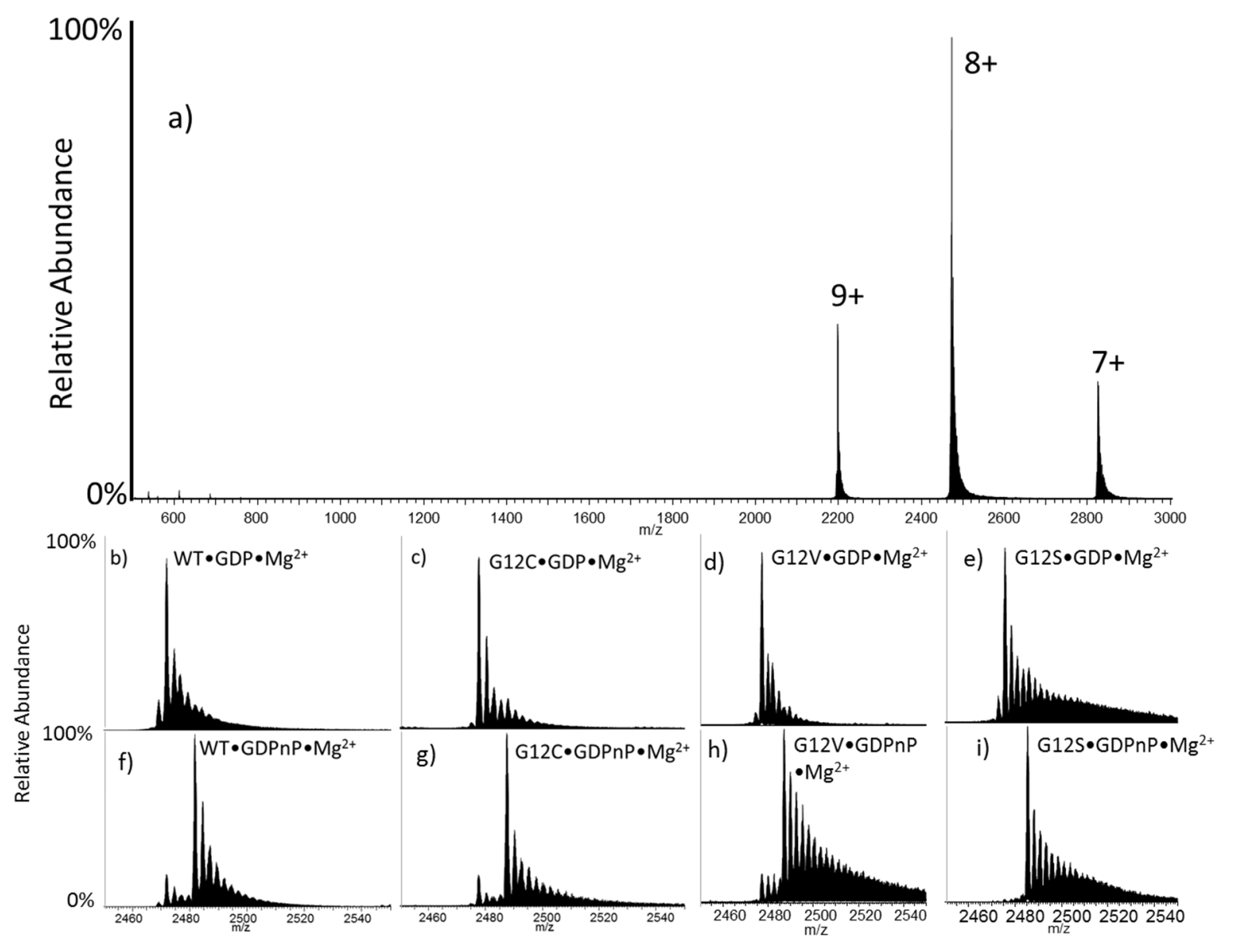


Figure S7. UVPD mass spectra of K-Ras (8+) activated using a single $3 \mathrm{~mJ}$ pulse for a) $\mathrm{WT} \bullet \mathrm{GDP} \bullet \mathrm{Mg} 2+, \mathrm{b}$ )

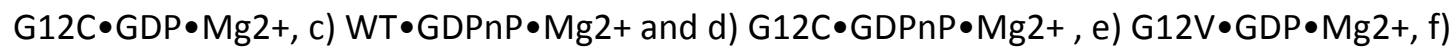

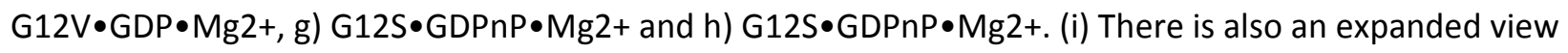
from $1400-1500 \mathrm{~m} / \mathrm{z}$ with selected ions labelled from panel (a).

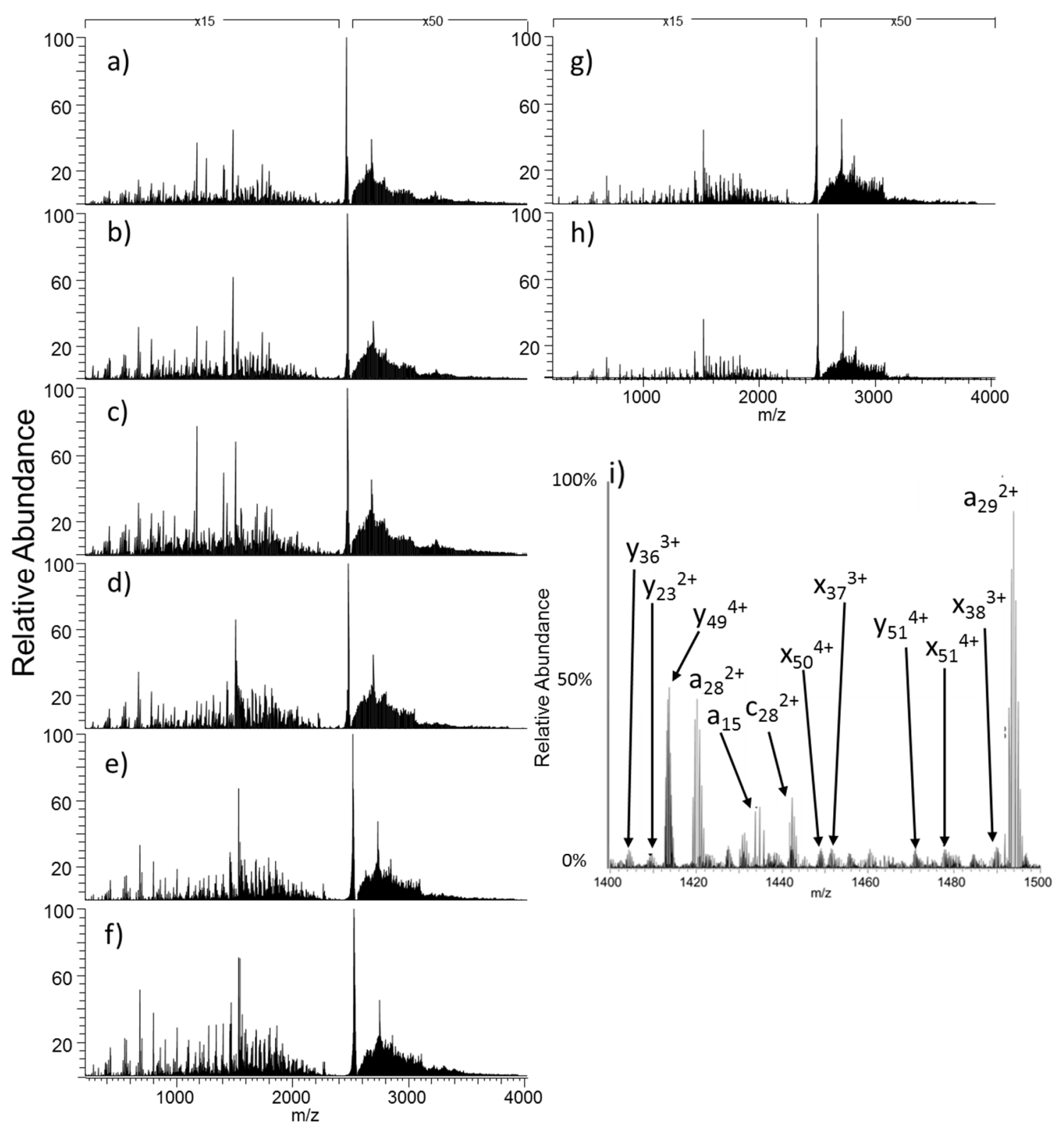


Figure S8. Deconvoluted UVPD mass spectra of WT K-Ras corresponding to the spectra in Figure

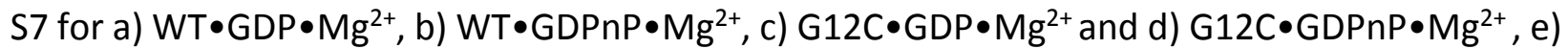
$\mathrm{G} 12 \mathrm{~V} \bullet \mathrm{GDP} \bullet \mathrm{Mg}^{2+}$, f) $\left.\mathrm{G} 12 \mathrm{~V} \bullet \mathrm{GDPnP} \bullet \mathrm{Mg}^{2+}, \mathrm{g}\right) \mathrm{G} 12 \mathrm{~S} \bullet \mathrm{GDP} \bullet \mathrm{Mg}^{2+}$ and h) G12S・GDPnP$\bullet \mathrm{Mg}^{2+}$. The asterisks indicate deconvolution artifacts at half the mass of the precursor due to incorrect deconvolution of a 4+ precursor ion when it is the non-dissociated 8+ precursor ion. Complete lists of identified ions are provided in supplementary Excel files.
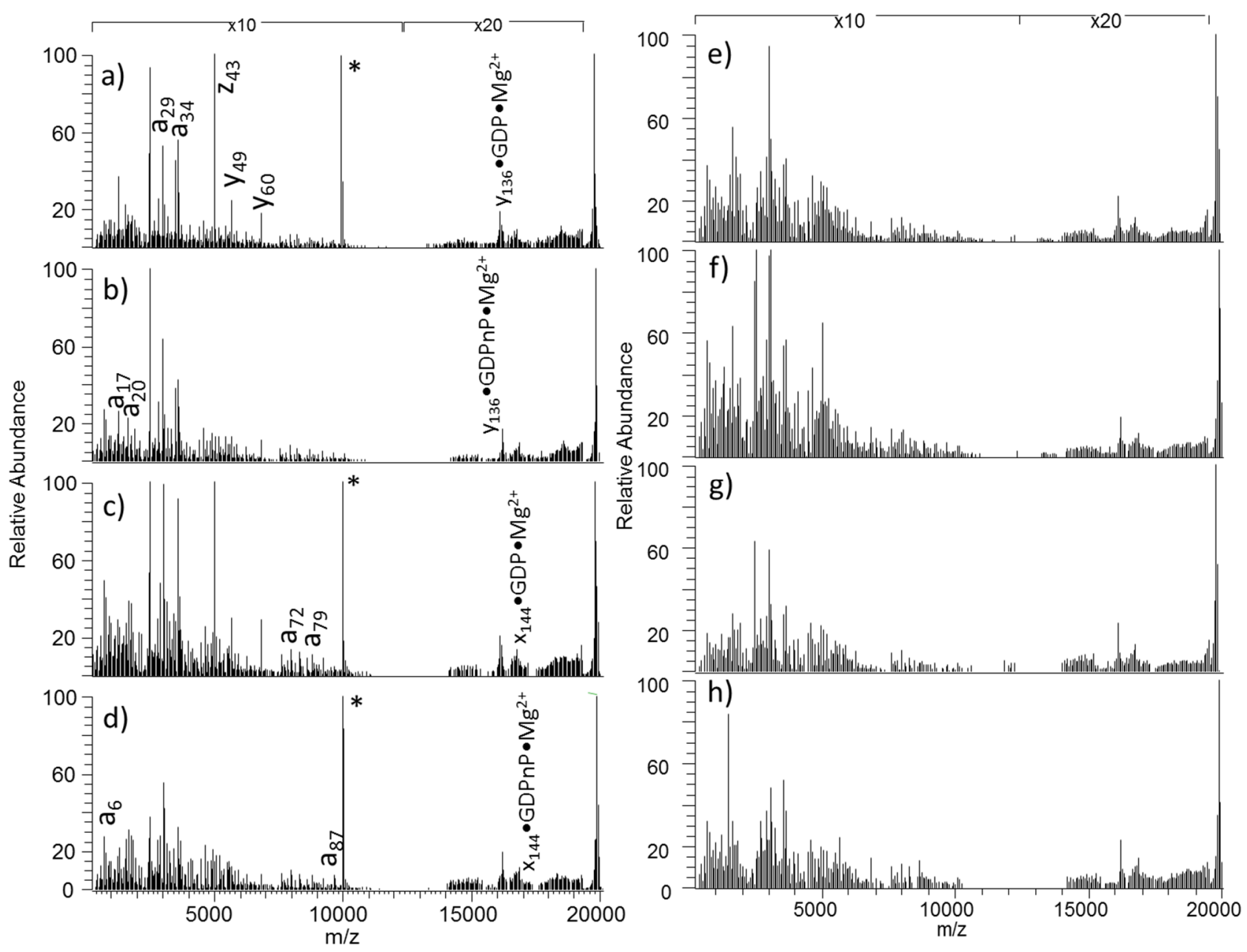
Figure S9. Number of holo fragment ions (i.e. ones retaining GDP or GDPnP) identified relative to the mass shift of the identified fragment ions with and without $\mathrm{Mg}^{2+}$ combined with GDP or GDPnP for WT K-Ras and G12C. Standard deviations of the number of identified fragments are shown averaged for four replicates. The black vertical lines on the right-hand graphs show the expected mass shift. The blue shaded region indicates the mass shifts that were searched for the complexes. Exhaustive searches revealed that more products were successfully identified containing both ligands (nucleotide plus $\mathrm{Mg}^{2+}$ ) for all protein complexes of interest and thus these products were targeted in the present study. The product ions that were specifically searched for the protein/GDP complexes included those incorporating a mass shift of 462.9781-464.9937 Da (relative to the apo fragment ions), and those searched for the protein/GDPnP complexes included those with a mass shift of 541.9604-543.9760 Da.

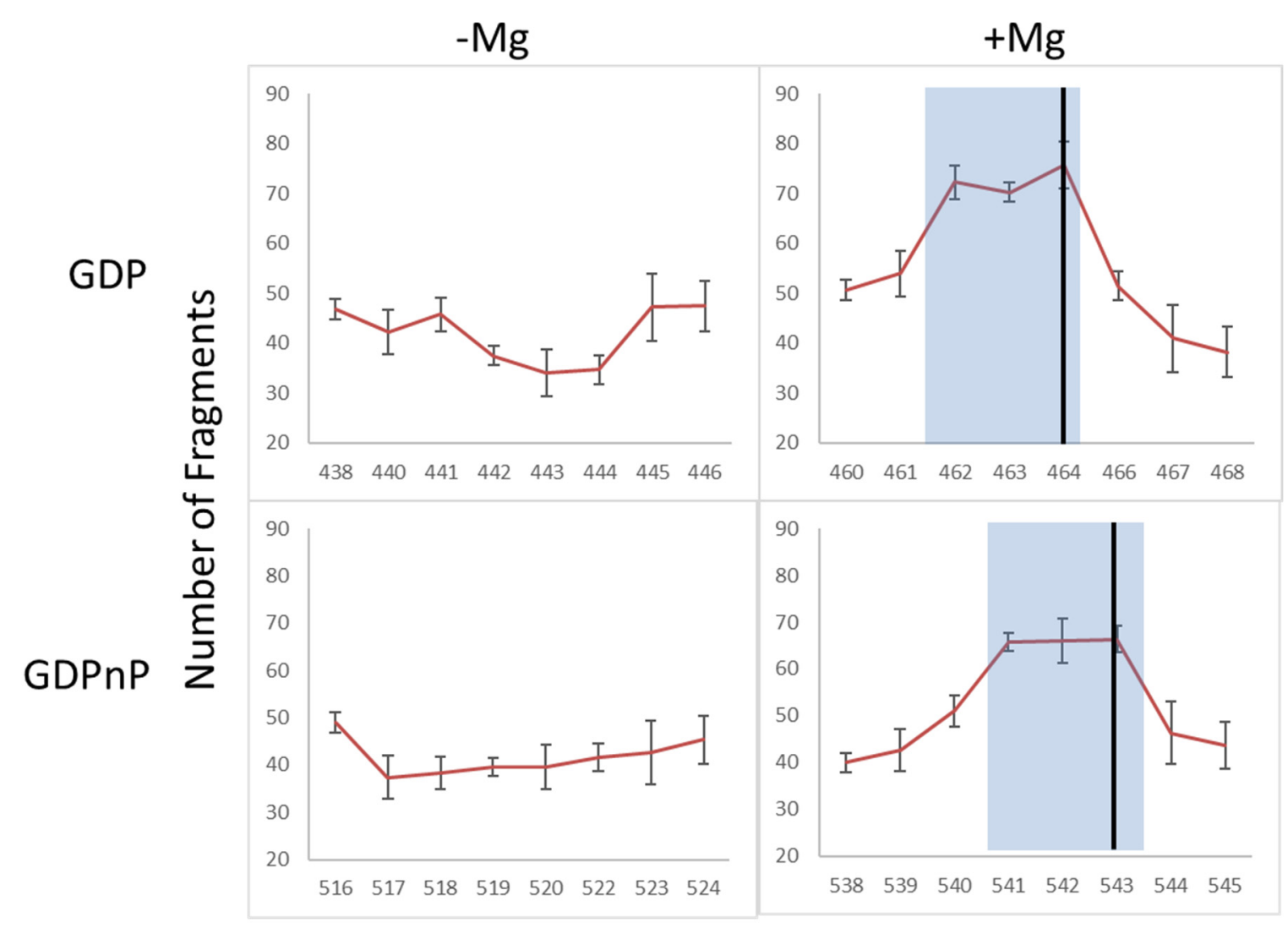

Mass Shift (Da) Searched 
Figure S10. (a,b,c,d) Relative abundances of the holo (ligand-containing) fragment ions produced upon UVPD of G12V and G12S complexes, color coded as N-terminal holo fragments (blue traces) and C-terminal holo fragments (red traces). The abundances are plotted relative to the amino acid sequence along the $x$-axis to convey the relative efficiencies of backbone cleavages adjacent to each residue. Shaded regions (blue and lavender shading) highlight the regions of the most notable changes in UVPD fragmentation efficiencies between the GDP and GDPnP complexes and are expanded in Figure S11.
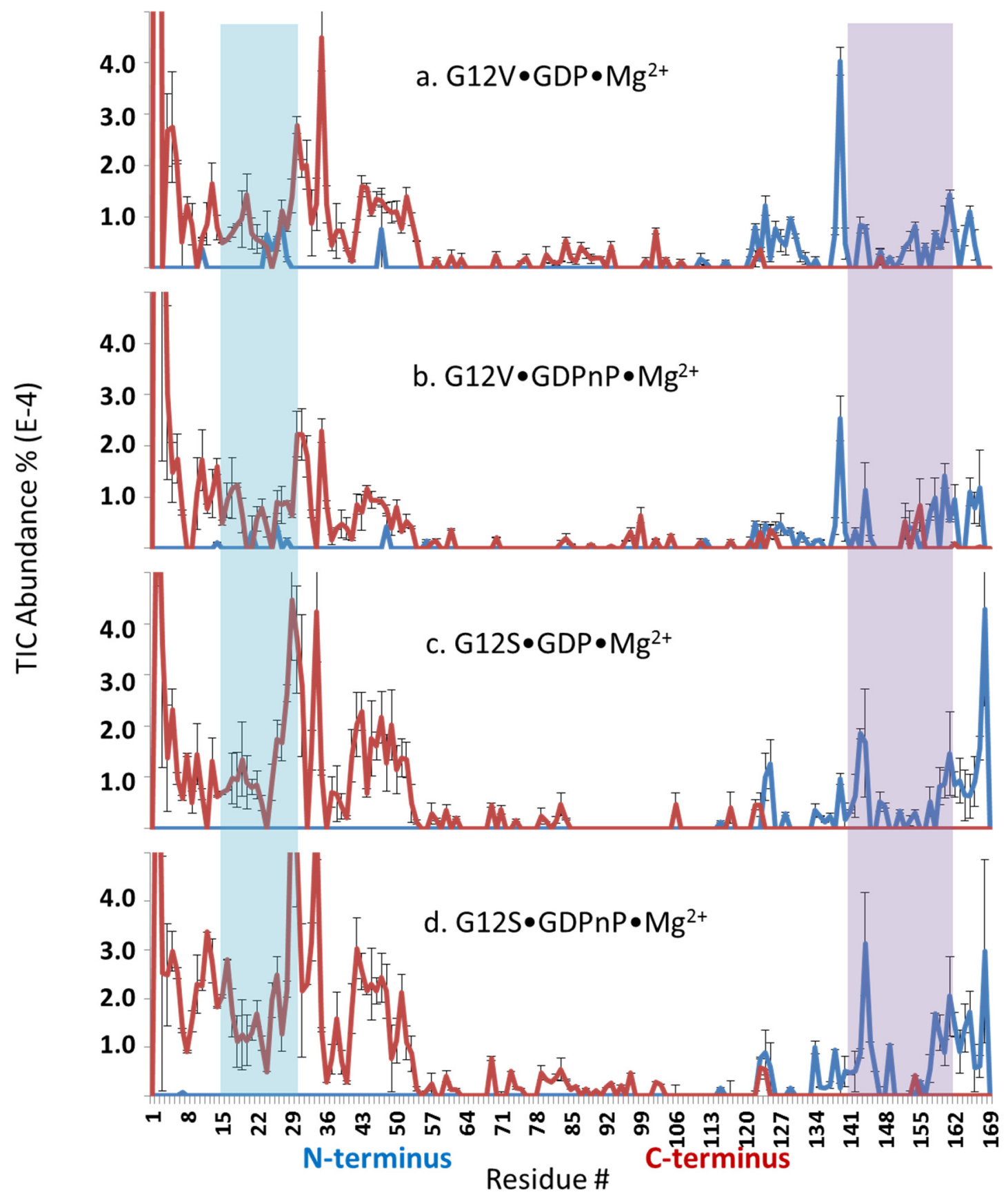
Figure S11. Expanded regions from Figure 1 and Figure S10. Relative abundances of the holofragment ions produced upon UVPD of WT-Kras and G12C complexes, color coded as Nterminal fragments (blue traces) and C-terminal fragments (red traces).

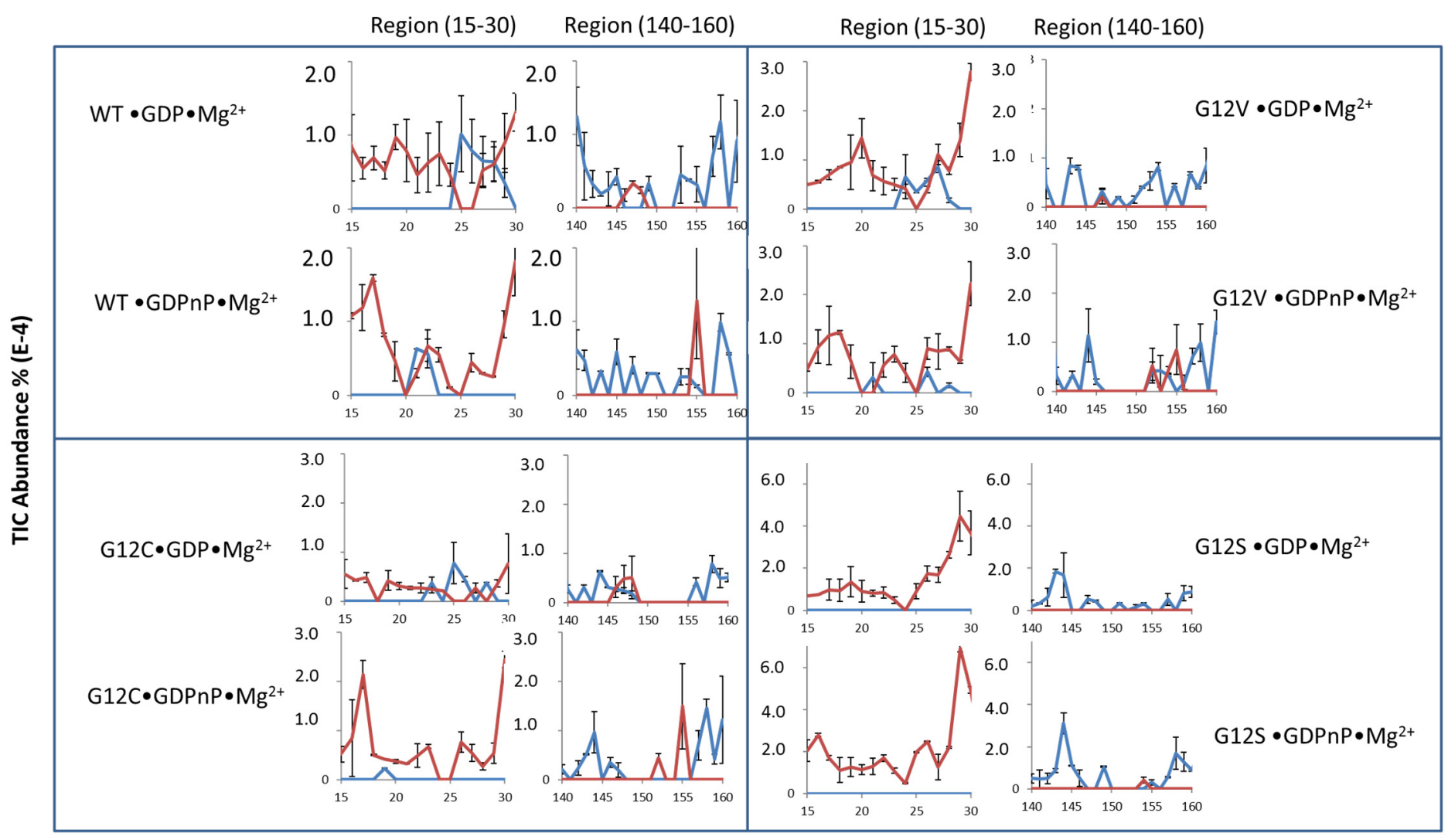

N-terminus Residue \# C-terminus 
Figure S12. Relative ion abundances of the summed holo and apo fragment ions produced upon UVPD of complexes containing (a) WT K-Ras, (b) G12C, (c) G12V, or (d) G12S and GDP• $\mathrm{Mg}^{2+}$ (blue data trend) or GDPnP $\bullet \mathrm{Mg}^{2+}$ (red data trend). Fragment ions from both $\mathrm{N}$-terminal and $\mathrm{C}$-terminal cleavages on either side of each residue are summed. Both holo and apo fragment ions are included. The pink, gray, and blue shaded regions indicate regions of change specific to the G12X complexes in comparison to the WT K-Ras complexes. Upward or downward arrows adjacent to the shaded sections indicate those regions for which UVPD is enhanced or suppressed upon ligand exchange from GDP to GDPnP. Relevant loops and switches are labelled underneath the $x-$ axis using colors corresponding to Supplemental Figure 5. Calculated difference plots are shown in Figure 3.

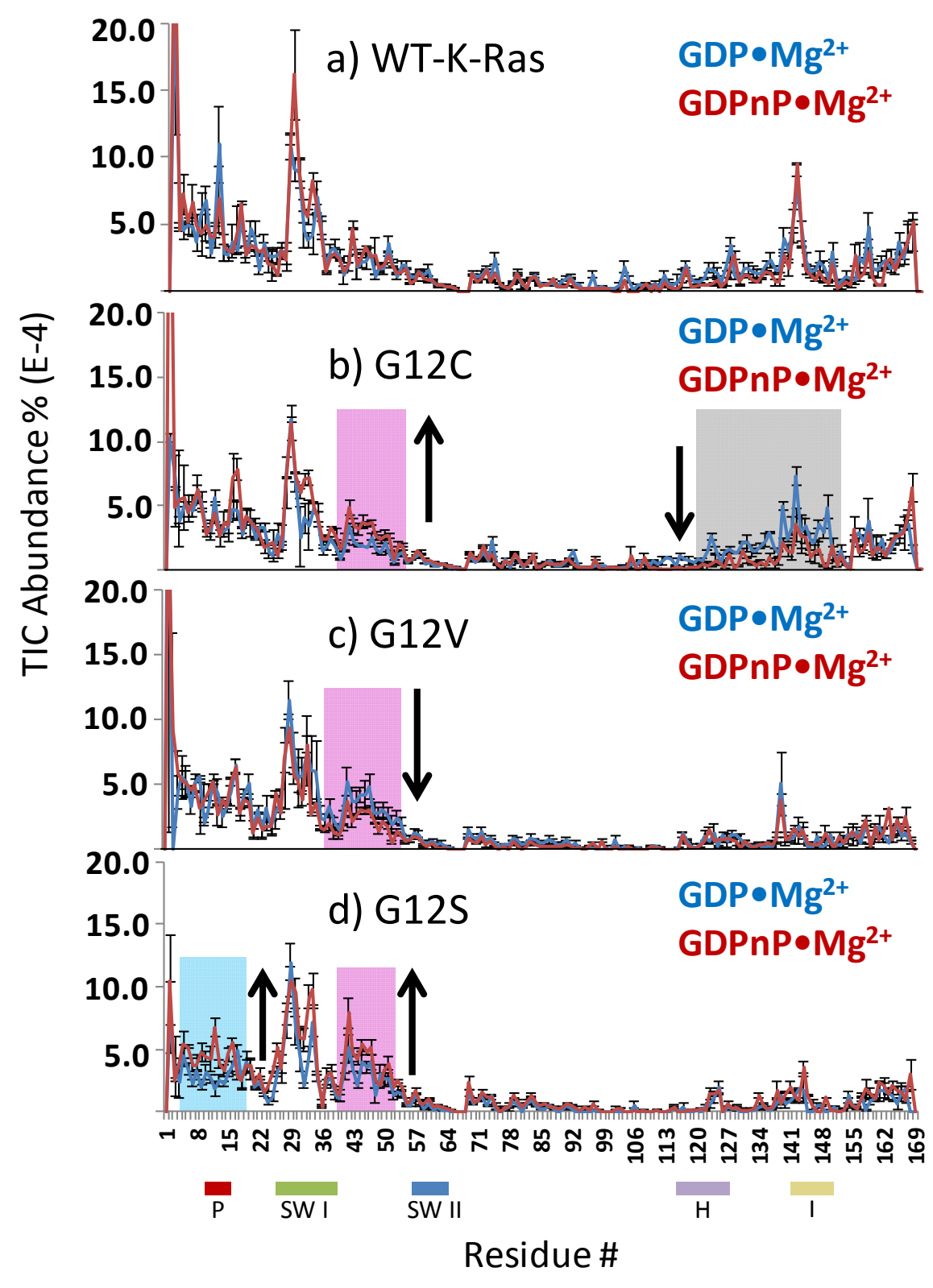


Table S1A. Summary of UVPD mass spectrum for G12C•GDP complex: apo fragment ions

Column "Ions" is the fragment ion identity $(a, b, c, x, y, z)$ along with the number of residues in the fragment ion. A plus or minus sign indicates the fragment ion has one additional hydrogen atom or one less hydrogen atom than a conventional fragment ion. Column "Ion Type" is the type of fragment ion $(a, b, c, x, y, z)$. A plus sign indicates a fragment ion $(a, x)$ with an additional hydrogen atom; a minus sign indicates a fragment ion $(y)$ that is missing one hydrogen atom (the addition of one hydrogen atom for $a / x$ ions is not uncommon in UVPD mass spectra of intact proteins); Column "Residue" is the number of residues in the fragment ion. Column "Theoretical Mass" is the theoretical mass of the fragment ion; Column "Experimental Mass" is the experimentally measured mass of the fragment ion. Column "Delta mass" is the mass difference between the theoretical and experimental masses; and column "ppm error" is the mass error in ppm. The "apo" table (Table S1A) provides the fragment ions that do not retain the ligand. Tables S1B, S1C, and S1D provide the fragment ions that incorporate the ligand based on the mass shift corresponding to the retained ligand (with or without an additional hydrogen atom).

\begin{tabular}{|c|c|c|c|c|c|c|}
\hline Ion & $\frac{\text { Ion }}{\text { Type }}$ & Residue & $\begin{array}{l}\text { Theoretical } \\
\text { Mass }\end{array}$ & $\frac{\text { Experimental }}{\text { Mass }}$ & $\Delta$ mass & $\begin{array}{l}\text { ppm } \\
\text { error }\end{array}$ \\
\hline$\overline{a+43}$ & $\overline{a+}$ & 473 & 4741.401 & 4741.44 & $\overline{0.039184}$ & $\overline{8.264217}$ \\
\hline$a+44$ & $a+$ & 44 & 4869.459 & 4869.498 & 0.038343 & 7.874173 \\
\hline$a+46$ & $a+$ & 46 & 5067.596 & 5067.636 & 0.039585 & 7.811389 \\
\hline$a+47$ & $a+$ & 47 & 5180.68 & 5180.718 & 0.037434 & 7.225686 \\
\hline$a+49$ & $a+$ & 49 & 5352.729 & 5352.766 & 0.037496 & 7.005019 \\
\hline$a+5$ & $a+$ & 5 & 554.2285 & 554.2339 & 0.005413 & 9.766673 \\
\hline$a+50$ & $a+$ & 50 & 5481.771 & 5481.817 & 0.045247 & 8.254078 \\
\hline$a+51$ & $a+$ & 51 & 5582.819 & 5582.865 & 0.045981 & 8.236156 \\
\hline$a+59$ & $a+$ & 59 & 6469.266 & 6469.312 & 0.04589 & 7.093535 \\
\hline$a+6$ & $a+$ & 6 & 682.3234 & 682.3302 & 0.006767 & 9.917537 \\
\hline$a+61$ & $a+$ & 61 & 6597.325 & 6597.375 & 0.050553 & 7.662647 \\
\hline$a+66$ & $a+$ & 66 & 7233.564 & 7233.567 & 0.002887 & 0.399107 \\
\hline$a+7$ & $a+$ & 7 & 795.4075 & 795.4157 & 0.008217 & 10.33051 \\
\hline$a+71$ & $a+$ & 71 & 7834.828 & 7834.888 & 0.059946 & 7.651217 \\
\hline$a+74$ & $a+$ & 74 & 8285.033 & 8285.103 & 0.070517 & 8.511369 \\
\hline$a+8$ & $a+$ & 8 & 894.4759 & 894.485 & 0.009143 & 10.22159 \\
\hline$a+82$ & $a+$ & 82 & 9091.396 & 9091.482 & 0.086042 & 9.464109 \\
\hline$a+87$ & $a+$ & 87 & 9650.672 & 9650.744 & 0.072686 & 7.531701 \\
\hline$a+9$ & $a+$ & 9 & 993.5443 & 993.5541 & 0.009764 & 9.827411 \\
\hline$a+93$ & $a+$ & 93 & 10357.98 & 10358.08 & 0.097372 & 9.400668 \\
\hline$a+94$ & $a+$ & 94 & 10471.07 & 10471.15 & 0.080803 & 7.716784 \\
\hline a10 & a & 10 & 1091.605 & 1091.616 & 0.010641 & 9.748033 \\
\hline a127 & $a$ & 127 & 14315.01 & 14314.96 & -0.04984 & -3.48194 \\
\hline a13 & a & 13 & 1322.673 & 1322.685 & 0.012339 & 9.328839 \\
\hline
\end{tabular}




\begin{tabular}{|c|c|c|c|c|c|c|}
\hline a15 & $a$ & 15 & 1478.763 & 1478.776 & 0.013947 & 9.431535 \\
\hline a16 & a & 16 & 1535.784 & 1535.797 & 0.012948 & 8.430873 \\
\hline a169 & a & 169 & 19162.55 & 19162.61 & 0.055881 & 2.916156 \\
\hline a17 & a & 17 & 1663.879 & 1663.895 & 0.015766 & 9.475449 \\
\hline a19 & a & 19 & 1821.948 & 1821.966 & 0.01784 & 9.791717 \\
\hline a21 & a & 21 & 2036.08 & 2036.099 & 0.019645 & 9.648443 \\
\hline a26 & a & 26 & 2631.449 & 2631.474 & 0.024573 & 9.338201 \\
\hline a29 & a & 29 & 3029.619 & 3029.648 & 0.028809 & 9.509115 \\
\hline a35 & a & 35 & 3747.9 & 3747.935 & 0.03507 & 9.357239 \\
\hline a36 & a & 36 & 3848.948 & 3848.981 & 0.032997 & 8.572992 \\
\hline a37 & a & 37 & 3962.032 & 3962.071 & 0.038537 & 9.726574 \\
\hline a4 & a & 4 & 390.1573 & 390.1608 & 0.00354 & 9.073263 \\
\hline a41 & a & 41 & 4456.197 & 4456.233 & 0.036489 & 8.188372 \\
\hline a42 & a & 42 & 4612.298 & 4612.338 & 0.039796 & 8.628237 \\
\hline a45 & a & 45 & 4967.52 & 4967.56 & 0.039892 & 8.030566 \\
\hline a48 & a & 48 & 5294.699 & 5294.742 & 0.042123 & 7.955692 \\
\hline a5 & a & 5 & 553.2206 & 553.2259 & 0.005243 & 9.477231 \\
\hline a53 & a & 53 & 5797.904 & 5797.953 & 0.048324 & 8.334735 \\
\hline a54 & a & 54 & 5910.989 & 5911.038 & 0.049103 & 8.307071 \\
\hline a55 & a & 55 & 6026.015 & 6026.063 & 0.047188 & 7.830713 \\
\hline a56 & a & 56 & 6139.1 & 6139.152 & 0.052361 & 8.529101 \\
\hline a57 & a & 57 & 6252.184 & 6252.236 & 0.052476 & 8.393228 \\
\hline a58 & a & 58 & 6367.211 & 6367.234 & 0.023562 & 3.700522 \\
\hline a60 & a & 60 & 6539.295 & 6539.332 & 0.036869 & 5.638069 \\
\hline a62 & a & 62 & 6724.375 & 6724.419 & 0.043302 & 6.439557 \\
\hline a63 & a & 63 & 6853.418 & 6853.472 & 0.054418 & 7.940272 \\
\hline a64 & a & 64 & 6982.461 & 6982.523 & 0.062757 & 8.987806 \\
\hline a69 & a & 69 & 7590.735 & 7590.796 & 0.061005 & 8.036772 \\
\hline a70 & a & 70 & 7705.762 & 7705.817 & 0.05555 & 7.208892 \\
\hline a72 & a & 72 & 7996.883 & 7996.943 & 0.059128 & 7.39388 \\
\hline a73 & a & 73 & 8127.924 & 8127.977 & 0.052818 & 6.498338 \\
\hline a75 & a & 75 & 8385.073 & 8385.145 & 0.072166 & 8.606485 \\
\hline a76 & a & 76 & 8442.094 & 8442.172 & 0.077919 & 9.229819 \\
\hline a77 & a & 77 & 8571.137 & 8571.201 & 0.063897 & 7.454904 \\
\hline a78 & a & 78 & 8628.158 & 8628.224 & 0.06588 & 7.635465 \\
\hline a79 & a & 79 & 8775.227 & 8775.297 & 0.070834 & 8.072042 \\
\hline a8 & a & 8 & 893.4681 & 893.477 & 0.008911 & 9.973496 \\
\hline a80 & a & 80 & 8888.311 & 8888.393 & 0.081867 & 9.210637 \\
\hline a83 & a & 83 & 9237.457 & 9237.522 & 0.065496 & 7.090263 \\
\hline a85 & a & 85 & 9421.578 & 9421.658 & 0.080395 & 8.533072 \\
\hline a86 & a & 86 & 9535.621 & 9535.7 & 0.078886 & 8.27277 \\
\hline a88 & a & 88 & 9750.711 & 9750.781 & 0.070063 & 7.185424 \\
\hline a89 & a & 89 & 9878.806 & 9878.852 & 0.045782 & 4.634366 \\
\hline
\end{tabular}




\begin{tabular}{|c|c|c|c|c|c|c|}
\hline a90 & a & 90 & 9965.838 & 9965.901 & 0.062702 & 6.291693 \\
\hline a91 & a & 91 & 10112.91 & 10112.97 & 0.064604 & 6.388272 \\
\hline a92 & a & 92 & 10241.95 & 10242.03 & 0.082632 & 8.067995 \\
\hline a95 & a & 95 & 10607.12 & 10607.21 & 0.090838 & 8.563871 \\
\hline a96 & a & 96 & 10744.18 & 10744.26 & 0.085501 & 7.957891 \\
\hline a97 & a & 97 & 10907.24 & 10907.28 & 0.034064 & 3.123063 \\
\hline b11 & $b$ & 11 & 1176.621 & 1176.633 & 0.011484 & 9.76015 \\
\hline b12 & $b$ & 12 & 1247.658 & 1247.671 & 0.01221 & 9.786333 \\
\hline b13 & $b$ & 13 & 1350.668 & 1350.68 & 0.012908 & 9.556756 \\
\hline b131 & $b$ & 131 & 14771.24 & 14771.13 & -0.115 & -7.78553 \\
\hline b133 & $b$ & 133 & 15014.33 & 15014.32 & -0.00177 & -0.11762 \\
\hline b14 & $b$ & 14 & 1407.689 & 1407.702 & 0.012688 & 9.013354 \\
\hline b143 & $b$ & 143 & 16131.95 & 16131.86 & -0.09096 & -5.6385 \\
\hline b15 & $b$ & 15 & 1506.757 & 1506.772 & 0.01459 & 9.683045 \\
\hline b16 & b & 16 & 1563.779 & 1563.793 & 0.01437 & 9.189279 \\
\hline b164 & $b$ & 164 & 18512.16 & 18512.02 & -0.13339 & -7.20548 \\
\hline b17 & b & 17 & 1691.874 & 1691.891 & 0.016748 & 9.899083 \\
\hline b19 & $b$ & 19 & 1849.943 & 1849.961 & 0.018043 & 9.753274 \\
\hline b20 & b & 20 & 1963.027 & 1963.045 & 0.018089 & 9.21485 \\
\hline b21 & $b$ & 21 & 2064.075 & 2064.094 & 0.019603 & 9.497234 \\
\hline b23 & b & 23 & 2305.217 & 2305.238 & 0.020762 & 9.006526 \\
\hline b24 & $b$ & 24 & 2418.301 & 2418.324 & 0.022151 & 9.159735 \\
\hline b28 & b & 28 & 2910.546 & 2910.573 & 0.027488 & 9.444276 \\
\hline b3 & $b$ & 3 & 289.1096 & 289.1123 & 0.002631 & 9.100354 \\
\hline b33 & $b$ & 33 & 3563.816 & 3563.849 & 0.033763 & 9.473835 \\
\hline b39 & $b$ & 39 & 4234.097 & 4234.13 & 0.033418 & 7.892593 \\
\hline b4 & b & 4 & 418.1522 & 418.156 & 0.003772 & 9.020638 \\
\hline b43 & $b$ & 43 & 4768.388 & 4768.432 & 0.044346 & 9.299998 \\
\hline b48 & $b$ & 48 & 5322.694 & 5322.748 & 0.053189 & 9.992871 \\
\hline b49 & $b$ & 49 & 5379.716 & 5379.766 & 0.049763 & 9.250117 \\
\hline b5 & $b$ & 5 & 581.2156 & 581.2212 & 0.005628 & 9.683155 \\
\hline b52 & $b$ & 52 & 5712.815 & 5712.864 & 0.048724 & 8.528895 \\
\hline b58 & b & 58 & 6395.205 & 6395.256 & 0.050551 & 7.904515 \\
\hline b75 & $b$ & 75 & 8413.068 & 8413.084 & 0.016487 & 1.959689 \\
\hline b80 & $b$ & 80 & 8916.306 & 8916.392 & 0.08594 & 9.638521 \\
\hline b83 & $b$ & 83 & 9265.452 & 9265.535 & 0.083468 & 9.008519 \\
\hline b9 & $b$ & 9 & 1020.531 & 1020.541 & 0.009551 & 9.35885 \\
\hline c10 & c & 10 & 1136.626 & 1136.637 & 0.011155 & 9.814133 \\
\hline c13 & c & 13 & 1367.694 & 1367.707 & 0.013219 & 9.665175 \\
\hline c134 & c & 134 & 15144.44 & 15144.4 & -0.03389 & -2.23759 \\
\hline c14 & c & 14 & 1424.715 & 1424.728 & 0.012389 & 8.695772 \\
\hline c146 & c & 146 & 16466.1 & 16466.07 & -0.03543 & -2.15169 \\
\hline c149 & c & 149 & 16766.28 & 16766.15 & -0.13315 & -7.94147 \\
\hline
\end{tabular}




\begin{tabular}{|c|c|c|c|c|c|c|}
\hline c16 & c & 16 & 1580.805 & 1580.816 & 0.010898 & 6.893955 \\
\hline c17 & c & 17 & 1708.9 & 1708.91 & 0.009443 & 5.525776 \\
\hline c18 & c & 18 & 1795.932 & 1795.95 & 0.017697 & 9.853936 \\
\hline$c 20$ & c & 20 & 1980.053 & 1980.07 & 0.016644 & 8.405834 \\
\hline c22 & c & 22 & 2194.185 & 2194.206 & 0.020816 & 9.486893 \\
\hline$c 23$ & c & 23 & 2322.244 & 2322.265 & 0.021074 & 9.074844 \\
\hline c25 & c & 25 & 2548.412 & 2548.436 & 0.024584 & 9.646793 \\
\hline$c 26$ & c & 26 & 2676.47 & 2676.495 & 0.025086 & 9.372792 \\
\hline$c 27$ & c & 27 & 2790.513 & 2790.516 & 0.003105 & 1.112699 \\
\hline$c 28$ & c & 28 & 2927.572 & 2927.597 & 0.025101 & 8.573999 \\
\hline c30 & c & 30 & 3173.709 & 3173.738 & 0.028858 & 9.092831 \\
\hline c33 & c & 33 & 3580.842 & 3580.873 & 0.031252 & 8.727557 \\
\hline c34 & c & 34 & 3695.869 & 3695.853 & -0.01627 & -4.40221 \\
\hline c35 & c & 35 & 3792.922 & 3792.957 & 0.035462 & 9.349521 \\
\hline c36 & c & 36 & 3893.969 & 3894.005 & 0.035952 & 9.232739 \\
\hline c38 & c & 38 & 4136.096 & 4136.129 & 0.033081 & 7.998122 \\
\hline c39 & c & 39 & 4251.123 & 4251.105 & -0.01798 & -4.22971 \\
\hline c4 & c & 4 & 435.1785 & 435.1827 & 0.004175 & 9.593764 \\
\hline$c 40$ & c & 40 & 4338.155 & 4338.195 & 0.040029 & 9.227195 \\
\hline c41 & c & 41 & 4501.218 & 4501.206 & -0.01251 & -2.77947 \\
\hline$c 42$ & c & 42 & 4657.319 & 4657.365 & 0.046047 & 9.887018 \\
\hline c44 & c & 44 & 4913.473 & 4913.513 & 0.040212 & 8.184028 \\
\hline$c 47$ & c & 47 & 5224.694 & 5224.732 & 0.037838 & 7.242147 \\
\hline$c 50$ & c & 50 & 5525.785 & 5525.831 & 0.046229 & 8.366052 \\
\hline$c 54$ & c & 54 & 5956.01 & 5956.059 & 0.048998 & 8.226649 \\
\hline c58 & c & 58 & 6412.232 & 6412.27 & 0.038432 & 5.993545 \\
\hline c59 & c & 59 & 6513.279 & 6513.331 & 0.051769 & 7.948223 \\
\hline$c 60$ & c & 60 & 6584.317 & 6584.321 & 0.004471 & 0.679038 \\
\hline c70 & c & 70 & 7750.783 & 7750.824 & 0.041171 & 5.311851 \\
\hline c74 & c & 74 & 8329.046 & 8329.082 & 0.036009 & 4.323304 \\
\hline c79 & c & 79 & 8820.248 & 8820.266 & 0.017881 & 2.027267 \\
\hline c84 & c & 84 & 9353.515 & 9353.575 & 0.060249 & 6.441322 \\
\hline c88 & $c$ & 88 & 9795.733 & 9795.765 & 0.032368 & 3.304296 \\
\hline$x+12$ & $x+$ & 12 & 1562.913 & 1562.924 & 0.011235 & 7.188479 \\
\hline$x+14$ & $x+$ & 14 & 1873.045 & 1873.058 & 0.01365 & 7.287582 \\
\hline$x+15$ & $x+$ & 15 & 1944.082 & 1944.096 & 0.014137 & 7.271796 \\
\hline$x+21$ & $x+$ & 21 & 2614.385 & 2614.407 & 0.021597 & 8.26082 \\
\hline$x+22$ & $x+$ & 22 & 2715.433 & 2715.456 & 0.022501 & 8.286328 \\
\hline$x+23$ & $x+$ & 23 & 2843.528 & 2843.55 & 0.022085 & 7.766749 \\
\hline$x+25$ & $x+$ & 25 & 3001.597 & 3001.62 & 0.023331 & 7.772851 \\
\hline$x+27$ & $x+$ & 27 & 3231.687 & 3231.711 & 0.023367 & 7.230578 \\
\hline$x+29$ & $x+$ & 29 & 3491.84 & 3491.867 & 0.027348 & 7.831965 \\
\hline$x+31$ & $x+$ & 31 & 3701.977 & 3702.008 & 0.031148 & 8.413875 \\
\hline
\end{tabular}




\begin{tabular}{|c|c|c|c|c|c|c|}
\hline$x+32$ & $x+$ & 32 & 3758.998 & 3759.023 & 0.025144 & 6.689008 \\
\hline$x+33$ & $x+$ & 33 & 3922.061 & 3922.099 & 0.037985 & 9.68495 \\
\hline$x+35$ & $x+$ & 35 & 4165.195 & 4165.22 & 0.025376 & 6.092385 \\
\hline$x+36$ & $x+$ & 36 & 4236.232 & 4236.267 & 0.035386 & 8.353171 \\
\hline$x+37$ & $x+$ & 37 & 4349.316 & 4349.347 & 0.031404 & 7.220439 \\
\hline$x+38$ & $x+$ & 38 & 4464.343 & 4464.378 & 0.034981 & 7.835637 \\
\hline$x+39$ & $x+$ & 39 & 4592.401 & 4592.441 & 0.039803 & 8.667136 \\
\hline$x+40$ & $x+$ & 40 & 4663.438 & 4663.474 & 0.035727 & 7.661079 \\
\hline$x+41$ & $x+$ & 41 & 4791.497 & 4791.536 & 0.038548 & 8.045078 \\
\hline$x+42$ & $x+$ & 42 & 4919.592 & 4919.631 & 0.039132 & 7.954312 \\
\hline$x+43$ & $x+$ & 43 & 5020.64 & 5020.683 & 0.043613 & 8.686735 \\
\hline$x+44$ & $x+$ & 44 & 5135.667 & 5135.716 & 0.049022 & 9.545395 \\
\hline$x+45$ & $x+$ & 45 & 5234.735 & 5234.785 & 0.050384 & 9.624932 \\
\hline$x+48$ & $x+$ & 48 & 5578.916 & 5578.967 & 0.05167 & 9.26165 \\
\hline$x+50$ & $x+$ & 50 & 5789.053 & 5789.107 & 0.054499 & 9.414143 \\
\hline$x+52$ & $x+$ & 52 & 6007.089 & 6007.143 & 0.054013 & 8.991538 \\
\hline$x+55$ & $x+$ & 55 & 6306.248 & 6306.303 & 0.05482 & 8.692961 \\
\hline$x+58$ & $x+$ & 58 & 6617.469 & 6617.531 & 0.062455 & 9.437894 \\
\hline$x+60$ & $x+$ & 60 & 6845.562 & 6845.626 & 0.064122 & 9.36694 \\
\hline$x+61$ & $x+$ & 61 & 6944.631 & 6944.685 & 0.054604 & 7.862761 \\
\hline$x+63$ & $x+$ & 63 & 7188.7 & 7188.77 & 0.069547 & 9.674484 \\
\hline$x+64$ & $x+$ & 64 & 7275.732 & 7275.763 & 0.030681 & 4.216891 \\
\hline$x+66$ & $x+$ & 66 & 7518.854 & 7518.925 & 0.071278 & 9.479898 \\
\hline$x+71$ & $x+$ & 71 & 8143.261 & 8143.336 & 0.074471 & 9.145104 \\
\hline x10 & $x$ & 10 & 1347.773 & 1347.786 & 0.012988 & 9.636634 \\
\hline$x 11$ & $x$ & 11 & 1460.858 & 1460.871 & 0.013719 & 9.391059 \\
\hline$x 12$ & $x$ & 12 & 1561.905 & 1561.92 & 0.014739 & 9.436552 \\
\hline$x 125$ & $x$ & 125 & 14367.12 & 14367 & -0.1186 & -8.25496 \\
\hline x16 & $x$ & 16 & 2058.101 & 2058.12 & 0.019436 & 9.443657 \\
\hline$x 17$ & $x$ & 17 & 2173.128 & 2173.149 & 0.020817 & 9.579279 \\
\hline$x 20$ & $x$ & 20 & 2457.276 & 2457.3 & 0.023978 & 9.757958 \\
\hline$x 25$ & $x$ & 25 & 3000.589 & 3000.619 & 0.029448 & 9.814072 \\
\hline$x 29$ & $x$ & 29 & 3490.832 & 3490.865 & 0.033215 & 9.514924 \\
\hline x3 & $x$ & 3 & 429.2223 & 429.2262 & 0.003812 & 8.881178 \\
\hline x31 & $x$ & 31 & 3700.969 & 3700.999 & 0.030259 & 8.175967 \\
\hline$x 54$ & $x$ & 54 & 6248.219 & 6248.274 & 0.054808 & 8.77178 \\
\hline x56 & $x$ & 56 & 6404.309 & 6404.372 & 0.062922 & 9.824948 \\
\hline$x 57$ & $x$ & 57 & 6517.393 & 6517.443 & 0.049823 & 7.644621 \\
\hline x59 & $x$ & 59 & 6747.502 & 6747.565 & 0.06291 & 9.323451 \\
\hline x9 & $x$ & 9 & 1248.705 & 1248.717 & 0.012009 & 9.617163 \\
\hline y11 & y & 11 & 1434.878 & 1434.892 & 0.014 & 9.756925 \\
\hline y12 & y & 12 & 1535.926 & 1535.941 & 0.014649 & 9.537569 \\
\hline y140 & y & 140 & 16179.97 & 16179.85 & -0.12393 & -7.65978 \\
\hline
\end{tabular}




\begin{tabular}{|c|c|c|c|c|c|c|}
\hline$y-147$ & $y-$ & 147 & 17030.43 & 17030.28 & -0.14954 & -8.78063 \\
\hline$y-15$ & $y-$ & 15 & 1916.087 & 1916.101 & 0.014498 & 7.566479 \\
\hline y16 & $y$ & 16 & 2032.122 & 2032.141 & 0.019698 & 9.693317 \\
\hline$y-18$ & $y-$ & 18 & 2245.209 & 2245.226 & 0.016889 & 7.522253 \\
\hline y21 & $y$ & 21 & 2587.398 & 2587.409 & 0.010972 & 4.240553 \\
\hline y22 & y & 22 & 2688.446 & 2688.471 & 0.025107 & 9.338852 \\
\hline y23 & y & 23 & 2816.541 & 2816.567 & 0.026095 & 9.264911 \\
\hline y24 & y & 24 & 2887.578 & 2887.602 & 0.024093 & 8.343671 \\
\hline y25 & y & 25 & 2974.61 & 2974.632 & 0.021849 & 7.345164 \\
\hline$y-25$ & $y-$ & 25 & 2973.602 & 2973.628 & 0.025801 & 8.676693 \\
\hline y26 & $y$ & 26 & 3075.658 & 3075.682 & 0.024313 & 7.904976 \\
\hline$y-26$ & $y-$ & 26 & 3074.65 & 3074.672 & 0.022494 & 7.315965 \\
\hline$y-27$ & $y-$ & 27 & 3203.692 & 3203.721 & 0.028244 & 8.816087 \\
\hline y28 & $y$ & 28 & 3317.784 & 3317.811 & 0.026765 & 8.067131 \\
\hline$y-28$ & $y-$ & 28 & 3316.777 & 3316.803 & 0.026825 & 8.087681 \\
\hline$y-29$ & $y-$ & 29 & 3463.845 & 3463.865 & 0.020135 & 5.812914 \\
\hline y3 & $y$ & 3 & 403.2431 & 403.2467 & 0.003616 & 8.967296 \\
\hline y30 & y & 30 & 3561.906 & 3561.933 & 0.027706 & 7.778421 \\
\hline$y-30$ & $y-$ & 30 & 3560.898 & 3560.927 & 0.028824 & 8.094597 \\
\hline y31 & $y$ & 31 & 3674.99 & 3675.023 & 0.033724 & 9.176625 \\
\hline$y-31$ & $y-$ & 31 & 3673.982 & 3674.005 & 0.023208 & 6.316861 \\
\hline y32 & $y$ & 32 & 3732.011 & 3732.043 & 0.031568 & 8.45871 \\
\hline y35 & y & 35 & 4138.208 & 4138.244 & 0.036636 & 8.853108 \\
\hline$y-36$ & $y-$ & 36 & 4208.237 & 4208.274 & 0.037136 & 8.824606 \\
\hline$y-37$ & $y-$ & 37 & 4321.321 & 4321.361 & 0.040479 & 9.367282 \\
\hline y38 & $y$ & 38 & 4437.356 & 4437.396 & 0.040382 & 9.100465 \\
\hline$y-39$ & $y-$ & 39 & 4564.406 & 4564.447 & 0.041018 & 8.986499 \\
\hline y4 & $y$ & 4 & 540.302 & 540.3071 & 0.00513 & 9.49469 \\
\hline$y-40$ & $y-$ & 40 & 4635.443 & 4635.483 & 0.0396 & 8.542879 \\
\hline$y-41$ & $y-$ & 41 & 4763.502 & 4763.546 & 0.044009 & 9.238798 \\
\hline$y-42$ & $y-$ & 42 & 4891.597 & 4891.644 & 0.046657 & 9.5382 \\
\hline y43 & y & 43 & 4993.653 & 4993.694 & 0.041005 & 8.211424 \\
\hline$y-43$ & $y-$ & 43 & 4992.645 & 4992.679 & 0.034012 & 6.812428 \\
\hline$y-44$ & $y-$ & 44 & 5107.672 & 5107.722 & 0.050437 & 9.87476 \\
\hline y45 & $y$ & 45 & 5207.748 & 5207.792 & 0.043874 & 8.424755 \\
\hline$y-45$ & $y-$ & 45 & 5206.74 & 5206.792 & 0.051698 & 9.92906 \\
\hline$y-47$ & $y-$ & 47 & 5463.889 & 5463.938 & 0.04883 & 8.936864 \\
\hline y48 & y & 48 & 5551.929 & 5551.978 & 0.049014 & 8.828283 \\
\hline y49 & y & 49 & 5648.981 & 5649.024 & 0.0422 & 7.470373 \\
\hline$y-50$ & $y-$ & 50 & 5761.058 & 5761.112 & 0.054411 & 9.444625 \\
\hline$y-52$ & $y-$ & 52 & 5979.094 & 5979.15 & 0.056232 & 9.404775 \\
\hline y55 & $y$ & 55 & 6279.261 & 6279.319 & 0.057964 & 9.231023 \\
\hline$y-58$ & $y-$ & 58 & 6589.474 & 6589.54 & 0.065441 & 9.931146 \\
\hline
\end{tabular}




\begin{tabular}{|c|c|c|c|c|c|c|c|c|}
\hline$y-59$ & $y-$ & 59 & 6720.515 & \multicolumn{2}{|c|}{6720.569} & \multicolumn{2}{|c|}{0.054478} & 8.106229 \\
\hline y61 & $y$ & 61 & 6917.644 & \multicolumn{2}{|c|}{6917.693} & \multicolumn{2}{|c|}{0.049018} & 7.085939 \\
\hline$y-62$ & $y-$ & 62 & 7031.663 & \multicolumn{2}{|c|}{7031.728} & \multicolumn{2}{|c|}{0.065803} & 9.358104 \\
\hline y64 & $y$ & 64 & 7248.745 & \multicolumn{2}{|c|}{7248.809} & \multicolumn{2}{|c|}{0.063509} & 8.761379 \\
\hline z11 & $z$ & 11 & 1418.86 & \multicolumn{2}{|c|}{1418.872} & \multicolumn{2}{|c|}{0.012476} & 8.792978 \\
\hline z13 & $z$ & 13 & 1682.971 & \multicolumn{2}{|c|}{1682.986} & \multicolumn{2}{|c|}{0.015771} & 9.370931 \\
\hline z14 & z & 14 & 1830.039 & \multicolumn{2}{|c|}{1830.055} & \multicolumn{2}{|c|}{0.016453} & 8.990519 \\
\hline z15 & $z$ & 15 & 1901.076 & \multicolumn{2}{|c|}{1901.093} & \multicolumn{2}{|c|}{0.016819} & 8.847095 \\
\hline z159 & $z$ & 159 & 18144.01 & \multicolumn{2}{|c|}{18143.84} & \multicolumn{2}{|c|}{-0.17044} & -9.39401 \\
\hline z16 & $z$ & 16 & 2016.103 & \multicolumn{2}{|c|}{2016.119} & \multicolumn{2}{|c|}{0.016124} & 7.997607 \\
\hline z21 & $z$ & 21 & 2571.379 & \multicolumn{2}{|c|}{2571.402} & \multicolumn{2}{|c|}{0.022569} & 8.777001 \\
\hline z23 & z & 23 & 2800.522 & \multicolumn{2}{|c|}{2800.541} & 0.01 & 8728 & 6.687324 \\
\hline$z 24$ & $z$ & 24 & 2871.559 & 2871.5 & 586 & 0.02 & 6291 & 9.155653 \\
\hline z25 & $z$ & 25 & 2958.591 & 2958. & 616 & 0.0 & 2429 & 8.209988 \\
\hline z27 & $z$ & 27 & 3188.682 & 3188. & 711 & 0.02 & 9296 & 9.187496 \\
\hline$z 28$ & $z$ & 28 & 3301.766 & 3301. & 796 & 0.03 & 0882 & 9.353178 \\
\hline z29 & $z$ & 29 & 3448.834 & 3448.8 & 866 & 0.03 & 1734 & 9.201371 \\
\hline$z 3$ & $z$ & 3 & 387.2243 & 387.22 & 279 & 0.00 & 3551 & 9.170395 \\
\hline z32 & z & 32 & 3715.992 & 3716. & 008 & 0.01 & 5862 & 4.268577 \\
\hline z33 & $z$ & 33 & 3879.056 & 3879. & 088 & 0.03 & 2047 & 8.261547 \\
\hline z35 & $z$ & 35 & 4122.189 & 4122.2 & 223 & 0.03 & 4405 & 8.346294 \\
\hline z36 & z & 36 & 4193.226 & 4193.2 & 256 & 0.02 & 9766 & 7.098592 \\
\hline z37 & z & 37 & 4306.31 & 4306.3 & 347 & 0.03 & 7503 & 8.708848 \\
\hline z4 & $z$ & 4 & 524.2832 & $524.2 \varepsilon$ & 881 & 0.00 & 4914 & 9.372797 \\
\hline $\mathrm{z} 40$ & z & 40 & 4620.433 & 4620. & 464 & 0.03 & 1206 & 6.753913 \\
\hline z47 & z & 47 & 5448.878 & 5448. & 923 & 0.04 & 5309 & 8.31529 \\
\hline z9 & $z$ & 9 & 1206.707 & 1206. & 718 & 0.01 & 0893 & 9.027046 \\
\hline$a+43$ & $a+$ & 43 & 4741.401 & 4741.44 & 0.03 & 9184 & 8.26 & 4217 \\
\hline$a+44$ & $a+$ & 44 & 4869.459 & 4869.498 & 0.03 & 8343 & 7.87 & 4173 \\
\hline$a+46$ & $a+$ & 46 & 5067.596 & 5067.636 & 0.03 & 9585 & 7.81 & 1389 \\
\hline$a+47$ & $a+$ & 47 & 5180.68 & 5180.718 & 0.03 & 7434 & 7.225 & 5686 \\
\hline$a+49$ & $a+$ & 49 & 5352.729 & 5352.766 & 0.03 & 7496 & 7.005 & 5019 \\
\hline$a+5$ & $a+$ & 5 & 554.2285 & 554.2339 & 0.00 & 5413 & 9.766 & 5673 \\
\hline$a+50$ & $a+$ & 50 & 5481.771 & 5481.817 & 0.0 & 5247 & 8.25 & 4078 \\
\hline$a+51$ & $a+$ & 51 & 5582.819 & 5582.865 & 0.0 & 5981 & 8.236 & 5156 \\
\hline$a+59$ & $a+$ & 59 & 6469.266 & 6469.312 & & 4589 & 7.093 & 3535 \\
\hline$a+6$ & $a+$ & 6 & 682.3234 & 682.3302 & 0.00 & 6767 & 9.917 & 7537 \\
\hline$a+61$ & $a+$ & 61 & 6597.325 & 6597.375 & 0.05 & 0553 & 7.66 & 2647 \\
\hline$a+66$ & $a+$ & 66 & 7233.564 & 7233.567 & 0.00 & 2887 & 0.39 & 9107 \\
\hline$a+7$ & $a+$ & 7 & 795.4075 & 795.4157 & 0.0 & 8217 & 10.33 & 3051 \\
\hline$a+71$ & $a+$ & 71 & 7834.828 & 7834.888 & 0.0 & 9946 & 7.65 & 1217 \\
\hline$a+74$ & $a+$ & 74 & 8285.033 & 8285.103 & 0.07 & 0517 & 8.51 & 1369 \\
\hline$a+8$ & $a+$ & 8 & 894.4759 & 894.485 & 0.0 & 9143 & 10.22 & 2159 \\
\hline
\end{tabular}




\begin{tabular}{|c|c|c|c|c|c|c|}
\hline$a+82$ & $a+$ & 82 & 9091.396 & 9091.482 & 0.086042 & 9.464109 \\
\hline$a+87$ & $a+$ & 87 & 9650.672 & 9650.744 & 0.072686 & 7.531701 \\
\hline$a+9$ & $a+$ & 9 & 993.5443 & 993.5541 & 0.009764 & 9.827411 \\
\hline$a+93$ & $a+$ & 93 & 10357.98 & 10358.08 & 0.097372 & 9.400668 \\
\hline$a+94$ & $a+$ & 94 & 10471.07 & 10471.15 & 0.080803 & 7.716784 \\
\hline a10 & $a$ & 10 & 1091.605 & 1091.616 & 0.010641 & 9.748033 \\
\hline a127 & $a$ & 127 & 14315.01 & 14314.96 & -0.04984 & -3.48194 \\
\hline a13 & $a$ & 13 & 1322.673 & 1322.685 & 0.012339 & 9.328839 \\
\hline a15 & $a$ & 15 & 1478.763 & 1478.776 & 0.013947 & 9.431535 \\
\hline a16 & $a$ & 16 & 1535.784 & 1535.797 & 0.012948 & 8.430873 \\
\hline a169 & $a$ & 169 & 19162.55 & 19162.61 & 0.055881 & 2.916156 \\
\hline a17 & a & 17 & 1663.879 & 1663.895 & 0.015766 & 9.475449 \\
\hline a19 & $a$ & 19 & 1821.948 & 1821.966 & 0.01784 & 9.791717 \\
\hline a21 & a & 21 & 2036.08 & 2036.099 & 0.019645 & 9.648443 \\
\hline a26 & $a$ & 26 & 2631.449 & 2631.474 & 0.024573 & 9.338201 \\
\hline a29 & a & 29 & 3029.619 & 3029.648 & 0.028809 & 9.509115 \\
\hline a35 & $a$ & 35 & 3747.9 & 3747.935 & 0.03507 & 9.357239 \\
\hline a36 & a & 36 & 3848.948 & 3848.981 & 0.032997 & 8.572992 \\
\hline a37 & a & 37 & 3962.032 & 3962.071 & 0.038537 & 9.726574 \\
\hline a4 & a & 4 & 390.1573 & 390.1608 & 0.00354 & 9.073263 \\
\hline a41 & a & 41 & 4456.197 & 4456.233 & 0.036489 & 8.188372 \\
\hline a42 & a & 42 & 4612.298 & 4612.338 & 0.039796 & 8.628237 \\
\hline a45 & a & 45 & 4967.52 & 4967.56 & 0.039892 & 8.030566 \\
\hline a48 & a & 48 & 5294.699 & 5294.742 & 0.042123 & 7.955692 \\
\hline a5 & a & 5 & 553.2206 & 553.2259 & 0.005243 & 9.477231 \\
\hline a53 & a & 53 & 5797.904 & 5797.953 & 0.048324 & 8.334735 \\
\hline a54 & a & 54 & 5910.989 & 5911.038 & 0.049103 & 8.307071 \\
\hline a55 & a & 55 & 6026.015 & 6026.063 & 0.047188 & 7.830713 \\
\hline a56 & a & 56 & 6139.1 & 6139.152 & 0.052361 & 8.529101 \\
\hline a57 & a & 57 & 6252.184 & 6252.236 & 0.052476 & 8.393228 \\
\hline a58 & a & 58 & 6367.211 & 6367.234 & 0.023562 & 3.700522 \\
\hline a60 & a & 60 & 6539.295 & 6539.332 & 0.036869 & 5.638069 \\
\hline a62 & a & 62 & 6724.375 & 6724.419 & 0.043302 & 6.439557 \\
\hline a63 & a & 63 & 6853.418 & 6853.472 & 0.054418 & 7.940272 \\
\hline a64 & a & 64 & 6982.461 & 6982.523 & 0.062757 & 8.987806 \\
\hline a69 & a & 69 & 7590.735 & 7590.796 & 0.061005 & 8.036772 \\
\hline a70 & $a$ & 70 & 7705.762 & 7705.817 & 0.05555 & 7.208892 \\
\hline a72 & a & 72 & 7996.883 & 7996.943 & 0.059128 & 7.39388 \\
\hline a73 & a & 73 & 8127.924 & 8127.977 & 0.052818 & 6.498338 \\
\hline a75 & a & 75 & 8385.073 & 8385.145 & 0.072166 & 8.606485 \\
\hline a76 & a & 76 & 8442.094 & 8442.172 & 0.077919 & 9.229819 \\
\hline a77 & a & 77 & 8571.137 & 8571.201 & 0.063897 & 7.454904 \\
\hline a78 & a & 78 & 8628.158 & 8628.224 & 0.06588 & 7.635465 \\
\hline
\end{tabular}




\begin{tabular}{|c|c|c|c|c|c|c|}
\hline a79 & $a$ & 79 & 8775.227 & 8775.297 & 0.070834 & 8.072042 \\
\hline a8 & a & 8 & 893.4681 & 893.477 & 0.008911 & 9.973496 \\
\hline a80 & $a$ & 80 & 8888.311 & 8888.393 & 0.081867 & 9.210637 \\
\hline a83 & a & 83 & 9237.457 & 9237.522 & 0.065496 & 7.090263 \\
\hline a85 & a & 85 & 9421.578 & 9421.658 & 0.080395 & 8.533072 \\
\hline a86 & a & 86 & 9535.621 & 9535.7 & 0.078886 & 8.27277 \\
\hline a88 & $a$ & 88 & 9750.711 & 9750.781 & 0.070063 & 7.185424 \\
\hline a89 & a & 89 & 9878.806 & 9878.852 & 0.045782 & 4.634366 \\
\hline a90 & a & 90 & 9965.838 & 9965.901 & 0.062702 & 6.291693 \\
\hline a91 & a & 91 & 10112.91 & 10112.97 & 0.064604 & 6.388272 \\
\hline a92 & a & 92 & 10241.95 & 10242.03 & 0.082632 & 8.067995 \\
\hline a95 & a & 95 & 10607.12 & 10607.21 & 0.090838 & 8.563871 \\
\hline a96 & a & 96 & 10744.18 & 10744.26 & 0.085501 & 7.957891 \\
\hline a97 & a & 97 & 10907.24 & 10907.28 & 0.034064 & 3.123063 \\
\hline b11 & $b$ & 11 & 1176.621 & 1176.633 & 0.011484 & 9.76015 \\
\hline b12 & $b$ & 12 & 1247.658 & 1247.671 & 0.01221 & 9.786333 \\
\hline b13 & $b$ & 13 & 1350.668 & 1350.68 & 0.012908 & 9.556756 \\
\hline b131 & $b$ & 131 & 14771.24 & 14771.13 & -0.115 & -7.78553 \\
\hline b133 & $b$ & 133 & 15014.33 & 15014.32 & -0.00177 & -0.11762 \\
\hline b14 & $b$ & 14 & 1407.689 & 1407.702 & 0.012688 & 9.013354 \\
\hline b143 & $b$ & 143 & 16131.95 & 16131.86 & -0.09096 & -5.6385 \\
\hline b15 & $b$ & 15 & 1506.757 & 1506.772 & 0.01459 & 9.683045 \\
\hline b16 & $b$ & 16 & 1563.779 & 1563.793 & 0.01437 & 9.189279 \\
\hline b164 & $b$ & 164 & 18512.16 & 18512.02 & -0.13339 & -7.20548 \\
\hline b17 & $b$ & 17 & 1691.874 & 1691.891 & 0.016748 & 9.899083 \\
\hline b19 & $b$ & 19 & 1849.943 & 1849.961 & 0.018043 & 9.753274 \\
\hline b20 & $b$ & 20 & 1963.027 & 1963.045 & 0.018089 & 9.21485 \\
\hline b21 & $b$ & 21 & 2064.075 & 2064.094 & 0.019603 & 9.497234 \\
\hline b23 & $b$ & 23 & 2305.217 & 2305.238 & 0.020762 & 9.006526 \\
\hline b24 & $b$ & 24 & 2418.301 & 2418.324 & 0.022151 & 9.159735 \\
\hline b28 & $b$ & 28 & 2910.546 & 2910.573 & 0.027488 & 9.444276 \\
\hline b3 & $b$ & 3 & 289.1096 & 289.1123 & 0.002631 & 9.100354 \\
\hline b33 & $b$ & 33 & 3563.816 & 3563.849 & 0.033763 & 9.473835 \\
\hline b39 & $b$ & 39 & 4234.097 & 4234.13 & 0.033418 & 7.892593 \\
\hline b4 & $b$ & 4 & 418.1522 & 418.156 & 0.003772 & 9.020638 \\
\hline b43 & $b$ & 43 & 4768.388 & 4768.432 & 0.044346 & 9.299998 \\
\hline b48 & $b$ & 48 & 5322.694 & 5322.748 & 0.053189 & 9.992871 \\
\hline b49 & $b$ & 49 & 5379.716 & 5379.766 & 0.049763 & 9.250117 \\
\hline b5 & b & 5 & 581.2156 & 581.2212 & 0.005628 & 9.683155 \\
\hline b52 & b & 52 & 5712.815 & 5712.864 & 0.048724 & 8.528895 \\
\hline b58 & b & 58 & 6395.205 & 6395.256 & 0.050551 & 7.904515 \\
\hline b75 & $b$ & 75 & 8413.068 & 8413.084 & 0.016487 & 1.959689 \\
\hline b80 & $b$ & 80 & 8916.306 & 8916.392 & 0.08594 & 9.63852 \\
\hline
\end{tabular}




\begin{tabular}{|c|c|c|c|c|c|c|}
\hline b83 & $b$ & 83 & 9265.452 & 9265.535 & 0.083468 & 9.008519 \\
\hline b9 & b & 9 & 1020.531 & 1020.541 & 0.009551 & 9.35885 \\
\hline c10 & c & 10 & 1136.626 & 1136.637 & 0.011155 & 9.814133 \\
\hline c13 & c & 13 & 1367.694 & 1367.707 & 0.013219 & 9.665175 \\
\hline c134 & c & 134 & 15144.44 & 15144.4 & -0.03389 & -2.23759 \\
\hline c14 & c & 14 & 1424.715 & 1424.728 & 0.012389 & 8.695772 \\
\hline c146 & c & 146 & 16466.1 & 16466.07 & -0.03543 & -2.15169 \\
\hline c149 & c & 149 & 16766.28 & 16766.15 & -0.13315 & -7.94147 \\
\hline c16 & c & 16 & 1580.805 & 1580.816 & 0.010898 & 6.893955 \\
\hline c17 & c & 17 & 1708.9 & 1708.91 & 0.009443 & 5.525776 \\
\hline c18 & c & 18 & 1795.932 & 1795.95 & 0.017697 & 9.853936 \\
\hline c20 & c & 20 & 1980.053 & 1980.07 & 0.016644 & 8.405834 \\
\hline$c 22$ & c & 22 & 2194.185 & 2194.206 & 0.020816 & 9.486893 \\
\hline$c 23$ & c & 23 & 2322.244 & 2322.265 & 0.021074 & 9.074844 \\
\hline$c 25$ & c & 25 & 2548.412 & 2548.436 & 0.024584 & 9.646793 \\
\hline c26 & c & 26 & 2676.47 & 2676.495 & 0.025086 & 9.372792 \\
\hline$c 27$ & c & 27 & 2790.513 & 2790.516 & 0.003105 & 1.112699 \\
\hline c28 & c & 28 & 2927.572 & 2927.597 & 0.025101 & 8.573999 \\
\hline c30 & c & 30 & 3173.709 & 3173.738 & 0.028858 & 9.092831 \\
\hline c33 & c & 33 & 3580.842 & 3580.873 & 0.031252 & 8.727557 \\
\hline c34 & c & 34 & 3695.869 & 3695.853 & -0.01627 & -4.40221 \\
\hline c35 & c & 35 & 3792.922 & 3792.957 & 0.035462 & 9.349521 \\
\hline c36 & c & 36 & 3893.969 & 3894.005 & 0.035952 & 9.232739 \\
\hline c38 & c & 38 & 4136.096 & 4136.129 & 0.033081 & 7.998122 \\
\hline c39 & c & 39 & 4251.123 & 4251.105 & -0.01798 & -4.22971 \\
\hline c4 & c & 4 & 435.1785 & 435.1827 & 0.004175 & 9.593764 \\
\hline $\mathrm{c} 40$ & c & 40 & 4338.155 & 4338.195 & 0.040029 & 9.227195 \\
\hline c41 & c & 41 & 4501.218 & 4501.206 & -0.01251 & -2.77947 \\
\hline c42 & c & 42 & 4657.319 & 4657.365 & 0.046047 & 9.887018 \\
\hline c44 & c & 44 & 4913.473 & 4913.513 & 0.040212 & 8.184028 \\
\hline c47 & c & 47 & 5224.694 & 5224.732 & 0.037838 & 7.242147 \\
\hline$c 50$ & c & 50 & 5525.785 & 5525.831 & 0.046229 & 8.366052 \\
\hline c54 & c & 54 & 5956.01 & 5956.059 & 0.048998 & 8.226649 \\
\hline$c 58$ & c & 58 & 6412.232 & 6412.27 & 0.038432 & 5.993545 \\
\hline c59 & c & 59 & 6513.279 & 6513.331 & 0.051769 & 7.948223 \\
\hline$c 60$ & c & 60 & 6584.317 & 6584.321 & 0.004471 & 0.679038 \\
\hline c70 & c & 70 & 7750.783 & 7750.824 & 0.041171 & 5.311851 \\
\hline c74 & c & 74 & 8329.046 & 8329.082 & 0.036009 & 4.323304 \\
\hline c79 & c & 79 & 8820.248 & 8820.266 & 0.017881 & 2.027267 \\
\hline c84 & c & 84 & 9353.515 & 9353.575 & 0.060249 & 6.441322 \\
\hline c88 & $\mathrm{C}$ & 88 & 9795.733 & 9795.765 & 0.032368 & 3.304296 \\
\hline$x+12$ & $x+$ & 12 & 1562.913 & 1562.924 & 0.011235 & 7.188479 \\
\hline$x+14$ & $x+$ & 14 & 1873.045 & 1873.058 & 0.01365 & 7.287582 \\
\hline
\end{tabular}




\begin{tabular}{|c|c|c|c|c|c|c|}
\hline$x+15$ & $x+$ & 15 & 1944.082 & 1944.096 & 0.014137 & 7.271796 \\
\hline$x+21$ & $x+$ & 21 & 2614.385 & 2614.407 & 0.021597 & 8.26082 \\
\hline$x+22$ & $x+$ & 22 & 2715.433 & 2715.456 & 0.022501 & 8.286328 \\
\hline$x+23$ & $x+$ & 23 & 2843.528 & 2843.55 & 0.022085 & 7.766749 \\
\hline$x+25$ & $x+$ & 25 & 3001.597 & 3001.62 & 0.023331 & 7.772851 \\
\hline$x+27$ & $x+$ & 27 & 3231.687 & 3231.711 & 0.023367 & 7.230578 \\
\hline$x+29$ & $x+$ & 29 & 3491.84 & 3491.867 & 0.027348 & 7.831965 \\
\hline$x+31$ & $x+$ & 31 & 3701.977 & 3702.008 & 0.031148 & 8.413875 \\
\hline$x+32$ & $x+$ & 32 & 3758.998 & 3759.023 & 0.025144 & 6.689008 \\
\hline$x+33$ & $x+$ & 33 & 3922.061 & 3922.099 & 0.037985 & 9.68495 \\
\hline$x+35$ & $x+$ & 35 & 4165.195 & 4165.22 & 0.025376 & 6.092385 \\
\hline$x+36$ & $x+$ & 36 & 4236.232 & 4236.267 & 0.035386 & 8.353171 \\
\hline$x+37$ & $x+$ & 37 & 4349.316 & 4349.347 & 0.031404 & 7.220439 \\
\hline$x+38$ & $x+$ & 38 & 4464.343 & 4464.378 & 0.034981 & 7.835637 \\
\hline$x+39$ & $x+$ & 39 & 4592.401 & 4592.441 & 0.039803 & 8.667136 \\
\hline$x+40$ & $x+$ & 40 & 4663.438 & 4663.474 & 0.035727 & 7.661079 \\
\hline$x+41$ & $x+$ & 41 & 4791.497 & 4791.536 & 0.038548 & 8.045078 \\
\hline$x+42$ & $x+$ & 42 & 4919.592 & 4919.631 & 0.039132 & 7.954312 \\
\hline$x+43$ & $x+$ & 43 & 5020.64 & 5020.683 & 0.043613 & 8.686735 \\
\hline$x+44$ & $x+$ & 44 & 5135.667 & 5135.716 & 0.049022 & 9.545395 \\
\hline$x+45$ & $x+$ & 45 & 5234.735 & 5234.785 & 0.050384 & 9.624932 \\
\hline$x+48$ & $x+$ & 48 & 5578.916 & 5578.967 & 0.05167 & 9.26165 \\
\hline$x+50$ & $x+$ & 50 & 5789.053 & 5789.107 & 0.054499 & 9.414143 \\
\hline$x+52$ & $x+$ & 52 & 6007.089 & 6007.143 & 0.054013 & 8.991538 \\
\hline$x+55$ & $x+$ & 55 & 6306.248 & 6306.303 & 0.05482 & 8.692961 \\
\hline$x+58$ & $x+$ & 58 & 6617.469 & 6617.531 & 0.062455 & 9.437894 \\
\hline$x+60$ & $x+$ & 60 & 6845.562 & 6845.626 & 0.064122 & 9.36694 \\
\hline$x+61$ & $x+$ & 61 & 6944.631 & 6944.685 & 0.054604 & 7.862761 \\
\hline$x+63$ & $x+$ & 63 & 7188.7 & 7188.77 & 0.069547 & 9.674484 \\
\hline$x+64$ & $x+$ & 64 & 7275.732 & 7275.763 & 0.030681 & 4.216891 \\
\hline$x+66$ & $x+$ & 66 & 7518.854 & 7518.925 & 0.071278 & 9.479898 \\
\hline$x+71$ & $x+$ & 71 & 8143.261 & 8143.336 & 0.074471 & 9.145104 \\
\hline$x 10$ & $x$ & 10 & 1347.773 & 1347.786 & 0.012988 & 9.636634 \\
\hline$x 11$ & $x$ & 11 & 1460.858 & 1460.871 & 0.013719 & 9.391059 \\
\hline$x 12$ & $x$ & 12 & 1561.905 & 1561.92 & 0.014739 & 9.436552 \\
\hline x125 & $x$ & 125 & 14367.12 & 14367 & -0.1186 & -8.25496 \\
\hline$x 16$ & $x$ & 16 & 2058.101 & 2058.12 & 0.019436 & 9.443657 \\
\hline$x 17$ & $x$ & 17 & 2173.128 & 2173.149 & 0.020817 & 9.579279 \\
\hline$x 20$ & $x$ & 20 & 2457.276 & 2457.3 & 0.023978 & 9.757958 \\
\hline$x 25$ & $x$ & 25 & 3000.589 & 3000.619 & 0.029448 & 9.814072 \\
\hline$x 29$ & $x$ & 29 & 3490.832 & 3490.865 & 0.033215 & 9.514924 \\
\hline$x 3$ & $x$ & 3 & 429.2223 & 429.2262 & 0.003812 & 8.881178 \\
\hline x31 & $x$ & 31 & 3700.969 & 3700.999 & 0.030259 & 8.175967 \\
\hline
\end{tabular}




\begin{tabular}{|c|c|c|c|c|c|c|}
\hline x54 & $x$ & 54 & 6248.219 & 6248.274 & 0.054808 & 8.77178 \\
\hline$x 56$ & $x$ & 56 & 6404.309 & 6404.372 & 0.062922 & 9.824948 \\
\hline x57 & $x$ & 57 & 6517.393 & 6517.443 & 0.049823 & 7.644621 \\
\hline x59 & $x$ & 59 & 6747.502 & 6747.565 & 0.06291 & 9.323451 \\
\hline$x 9$ & $x$ & 9 & 1248.705 & 1248.717 & 0.012009 & 9.617163 \\
\hline y11 & $y$ & 11 & 1434.878 & 1434.892 & 0.014 & 9.756925 \\
\hline y12 & $y$ & 12 & 1535.926 & 1535.941 & 0.014649 & 9.537569 \\
\hline y140 & $y$ & 140 & 16179.97 & 16179.85 & -0.12393 & -7.65978 \\
\hline$y-147$ & $y-$ & 147 & 17030.43 & 17030.28 & -0.14954 & -8.78063 \\
\hline$y-15$ & $y-$ & 15 & 1916.087 & 1916.101 & 0.014498 & 7.566479 \\
\hline y16 & $y$ & 16 & 2032.122 & 2032.141 & 0.019698 & 9.693317 \\
\hline$y-18$ & $y-$ & 18 & 2245.209 & 2245.226 & 0.016889 & 7.522253 \\
\hline y21 & $y$ & 21 & 2587.398 & 2587.409 & 0.010972 & 4.240553 \\
\hline y22 & $y$ & 22 & 2688.446 & 2688.471 & 0.025107 & 9.338852 \\
\hline y23 & $y$ & 23 & 2816.541 & 2816.567 & 0.026095 & 9.264911 \\
\hline y24 & $y$ & 24 & 2887.578 & 2887.602 & 0.024093 & 8.343671 \\
\hline y25 & $y$ & 25 & 2974.61 & 2974.632 & 0.021849 & 7.345164 \\
\hline$y-25$ & $y-$ & 25 & 2973.602 & 2973.628 & 0.025801 & 8.676693 \\
\hline y26 & $y$ & 26 & 3075.658 & 3075.682 & 0.024313 & 7.904976 \\
\hline$y-26$ & $y-$ & 26 & 3074.65 & 3074.672 & 0.022494 & 7.315965 \\
\hline$y-27$ & $y-$ & 27 & 3203.692 & 3203.721 & 0.028244 & 8.816087 \\
\hline y28 & $y$ & 28 & 3317.784 & 3317.811 & 0.026765 & 8.067131 \\
\hline$y-28$ & $y-$ & 28 & 3316.777 & 3316.803 & 0.026825 & 8.087681 \\
\hline$y-29$ & $y-$ & 29 & 3463.845 & 3463.865 & 0.020135 & 5.812914 \\
\hline y3 & $y$ & 3 & 403.2431 & 403.2467 & 0.003616 & 8.967296 \\
\hline y30 & $y$ & 30 & 3561.906 & 3561.933 & 0.027706 & 7.778421 \\
\hline$y-30$ & $y-$ & 30 & 3560.898 & 3560.927 & 0.028824 & 8.094597 \\
\hline y31 & $y$ & 31 & 3674.99 & 3675.023 & 0.033724 & 9.176625 \\
\hline$y-31$ & $y-$ & 31 & 3673.982 & 3674.005 & 0.023208 & 6.316861 \\
\hline y32 & $y$ & 32 & 3732.011 & 3732.043 & 0.031568 & 8.45871 \\
\hline y35 & $y$ & 35 & 4138.208 & 4138.244 & 0.036636 & 8.853108 \\
\hline$y-36$ & $y-$ & 36 & 4208.237 & 4208.274 & 0.037136 & 8.824606 \\
\hline$y-37$ & $y-$ & 37 & 4321.321 & 4321.361 & 0.040479 & 9.367282 \\
\hline y38 & $y$ & 38 & 4437.356 & 4437.396 & 0.040382 & 9.100465 \\
\hline$y-39$ & $y-$ & 39 & 4564.406 & 4564.447 & 0.041018 & 8.986499 \\
\hline y4 & $y$ & 4 & 540.302 & 540.3071 & 0.00513 & 9.49469 \\
\hline$y-40$ & $y-$ & 40 & 4635.443 & 4635.483 & 0.0396 & 8.542879 \\
\hline$y-41$ & $y-$ & 41 & 4763.502 & 4763.546 & 0.044009 & 9.238798 \\
\hline$y-42$ & $y-$ & 42 & 4891.597 & 4891.644 & 0.046657 & 9.5382 \\
\hline y43 & $\mathrm{y}$ & 43 & 4993.653 & 4993.694 & 0.041005 & 8.211424 \\
\hline$y-43$ & $y-$ & 43 & 4992.645 & 4992.679 & 0.034012 & 6.812428 \\
\hline$y-44$ & $y-$ & 44 & 5107.672 & 5107.722 & 0.050437 & 9.87476 \\
\hline y45 & $y$ & 45 & 5207.748 & 5207.792 & 0.043874 & 8.424755 \\
\hline
\end{tabular}




\begin{tabular}{|c|c|c|c|c|c|c|}
\hline$y-45$ & $y-$ & 45 & 5206.74 & 5206.792 & 0.051698 & 9.92906 \\
\hline$y-47$ & $y-$ & 47 & 5463.889 & 5463.938 & 0.04883 & 8.936864 \\
\hline y48 & y & 48 & 5551.929 & 5551.978 & 0.049014 & 8.828283 \\
\hline y49 & y & 49 & 5648.981 & 5649.024 & 0.0422 & 7.470373 \\
\hline$y-50$ & $y-$ & 50 & 5761.058 & 5761.112 & 0.054411 & 9.444625 \\
\hline$y-52$ & $y-$ & 52 & 5979.094 & 5979.15 & 0.056232 & 9.404775 \\
\hline y55 & $y$ & 55 & 6279.261 & 6279.319 & 0.057964 & 9.231023 \\
\hline$y-58$ & $y-$ & 58 & 6589.474 & 6589.54 & 0.065441 & 9.931146 \\
\hline$y-59$ & $y-$ & 59 & 6720.515 & 6720.569 & 0.054478 & 8.106229 \\
\hline y61 & $y$ & 61 & 6917.644 & 6917.693 & 0.049018 & 7.085939 \\
\hline$y-62$ & $y-$ & 62 & 7031.663 & 7031.728 & 0.065803 & 9.358104 \\
\hline y64 & $y$ & 64 & 7248.745 & 7248.809 & 0.063509 & 8.761379 \\
\hline z11 & $z$ & 11 & 1418.86 & 1418.872 & 0.012476 & 8.792978 \\
\hline z13 & $z$ & 13 & 1682.971 & 1682.986 & 0.015771 & 9.370931 \\
\hline z14 & z & 14 & 1830.039 & 1830.055 & 0.016453 & 8.990519 \\
\hline z15 & z & 15 & 1901.076 & 1901.093 & 0.016819 & 8.847095 \\
\hline z159 & $z$ & 159 & 18144.01 & 18143.84 & -0.17044 & -9.39401 \\
\hline z16 & $z$ & 16 & 2016.103 & 2016.119 & 0.016124 & 7.997607 \\
\hline z21 & z & 21 & 2571.379 & 2571.402 & 0.022569 & 8.777001 \\
\hline$z 23$ & z & 23 & 2800.522 & 2800.541 & 0.018728 & 6.687324 \\
\hline$z 24$ & $z$ & 24 & 2871.559 & 2871.586 & 0.026291 & 9.155653 \\
\hline$z 25$ & z & 25 & 2958.591 & 2958.616 & 0.02429 & 8.209988 \\
\hline z27 & z & 27 & 3188.682 & 3188.711 & 0.029296 & 9.187496 \\
\hline$z 28$ & z & 28 & 3301.766 & 3301.796 & 0.030882 & 9.353178 \\
\hline z29 & z & 29 & 3448.834 & 3448.866 & 0.031734 & 9.201371 \\
\hline z3 & z & 3 & 387.2243 & 387.2279 & 0.003551 & 9.170395 \\
\hline z32 & z & 32 & 3715.992 & 3716.008 & 0.015862 & 4.268577 \\
\hline z33 & z & 33 & 3879.056 & 3879.088 & 0.032047 & 8.261547 \\
\hline z35 & $z$ & 35 & 4122.189 & 4122.223 & 0.034405 & 8.346294 \\
\hline z36 & z & 36 & 4193.226 & 4193.256 & 0.029766 & 7.098592 \\
\hline z37 & z & 37 & 4306.31 & 4306.347 & 0.037503 & 8.708848 \\
\hline z4 & z & 4 & 524.2832 & 524.2881 & 0.004914 & 9.372797 \\
\hline z40 & z & 40 & 4620.433 & 4620.464 & 0.031206 & 6.753913 \\
\hline z47 & z & 47 & 5448.878 & 5448.923 & 0.045309 & 8.31529 \\
\hline z9 & z & 9 & 1206.707 & 1206.718 & 0.010893 & 9.027046 \\
\hline
\end{tabular}


Table S1B. Summary of UVPD mass spectrum for G12C•GDP complex: fragment ions incorporating mass shift of +464.9937 Da (GDP)

\begin{tabular}{|c|c|c|c|c|c|c|}
\hline & $\underline{\text { Ion }}$ & & Theoretical & Experimental & & \\
\hline Ion & Type & Residue & Mass & Mass & $\Delta$ mass & ppm error \\
\hline c5 & c & 5 & 1063.236 & 1063.239 & 0.003145 & 2.957952 \\
\hline c13 & c & 13 & 1832.688 & 1832.697 & 0.009631 & 5.255124 \\
\hline$a+14$ & $a+$ & 14 & 1845.696 & 1845.706 & 0.010716 & 5.805924 \\
\hline c125 & c & 125 & 14610.93 & 14611.03 & 0.10165 & 6.957122 \\
\hline a136 & a & 136 & 15791.55 & 15791.61 & 0.068297 & 4.324909 \\
\hline$a+141$ & $a+$ & 141 & 16309.81 & 16309.82 & 0.010203 & 0.625573 \\
\hline a146 & a & 146 & 16886.08 & 16886.23 & 0.156147 & 9.247087 \\
\hline a147 & a & 147 & 16957.11 & 16957.15 & 0.040912 & 2.412675 \\
\hline b156 & $b$ & 156 & 17955.59 & 17955.76 & 0.168812 & 9.40164 \\
\hline a157 & a & 157 & 18074.66 & 18074.79 & 0.126079 & 6.975455 \\
\hline b161 & $b$ & 161 & 18578.92 & 18579.03 & 0.11036 & 5.940065 \\
\hline a161 & a & 161 & 18550.93 & 18551.09 & 0.158253 & 8.530733 \\
\hline a166 & a & 166 & 19233.35 & 19233.47 & 0.116503 & 6.057343 \\
\hline z17 & $z$ & 17 & 2596.124 & 2596.115 & -0.00859 & -3.30955 \\
\hline y19 & y & 19 & 2768.232 & 2768.218 & -0.01469 & -5.30591 \\
\hline$x+20$ & $x+$ & 20 & 2923.278 & 2923.258 & -0.02032 & -6.95077 \\
\hline z45 & $z$ & 45 & 5656.723 & 5656.747 & 0.024335 & 4.301961 \\
\hline z48 & $z$ & 48 & 6000.904 & 6000.929 & 0.025156 & 4.192035 \\
\hline$x 48$ & $x$ & 48 & 6042.902 & 6042.95 & 0.048122 & 7.963393 \\
\hline$x+63$ & $x+$ & 63 & 7653.694 & 7653.752 & 0.05778 & 7.549292 \\
\hline z64 & $z$ & 64 & 7697.72 & 7697.788 & 0.068096 & 8.846256 \\
\hline$y-68$ & $y-$ & 68 & 8211.022 & 8211.102 & 0.079513 & 9.683694 \\
\hline$x 71$ & $x$ & 71 & 8607.247 & 8607.24 & -0.00748 & -0.86927 \\
\hline$x+78$ & $x+$ & 78 & 9558.691 & 9558.7 & 0.008804 & 0.921043 \\
\hline z81 & $z$ & 81 & 9878.828 & 9878.852 & 0.024217 & 2.451404 \\
\hline$x 84$ & $x$ & 84 & 10264.01 & 10264.01 & 0.002797 & 0.272506 \\
\hline$y-121$ & $y^{-}$ & 121 & 14420.92 & 14421.01 & 0.085305 & 5.915365 \\
\hline x122 & $x$ & 122 & 14504.93 & 14505.01 & 0.075709 & 5.219535 \\
\hline$x+123$ & $x+$ & 123 & 14620.97 & 14621.03 & 0.064077 & 4.382539 \\
\hline$y-125$ & $y-$ & 125 & 14805.13 & 14805.19 & 0.062342 & 4.210841 \\
\hline x127 & $x$ & 127 & 15059.24 & 15059.32 & 0.076133 & 5.055567 \\
\hline y133 & y & 133 & 15811.62 & 15811.63 & 0.01163 & 0.735535 \\
\hline y135 & y & 135 & 16025.75 & 16025.73 & -0.02617 & -1.63293 \\
\hline$y-136$ & $y-$ & 136 & 16121.8 & 16121.88 & 0.083973 & 5.208664 \\
\hline$x+141$ & $x+$ & 141 & 16771.02 & 16771.1 & 0.081063 & 4.833514 \\
\hline$y-148$ & $y-$ & 148 & 17623.48 & 17623.5 & 0.018152 & 1.029991 \\
\hline z149 & $z$ & 149 & 17721.55 & 17721.58 & 0.024182 & 1.364553 \\
\hline
\end{tabular}




$\begin{array}{llrrrrr}x 154 & x & 154 & 18263.85 & 18263.95 & 0.105556 & 5.779505 \\ x+156 & x+ & 156 & 18420.95 & 18421.03 & 0.085742 & 4.654591 \\ y-157 & y- & 157 & 18449.97 & 18450.1 & 0.129021 & 6.99302 \\ x+163 & x+ & 163 & 19006.24 & 19006.26 & 0.024447 & 1.28626 \\ z 164 & z & 164 & 19076.32 & 19076.41 & 0.089796 & 4.707198 \\ x 164 & x & 164 & 19118.32 & 19118.42 & 0.105438 & 5.515025\end{array}$


Table S1C. Summary of UVPD mass spectrum for G12C•GDP complex: fragment ions incorporating mass shift of +462.9781 Da (GDP)

\begin{tabular}{|c|c|c|c|c|c|c|}
\hline Ion & $\frac{\text { Ion }}{\text { Tyne }}$ & Residue & Theoretical & Experimental & Amass & $\frac{\mathrm{ppm}}{\mathrm{error}}$ \\
\hline a1 & a & 1 & $\overline{494.0046}$ & 494 & $\overline{-0.00464}$ & $\overline{-9.40275}$ \\
\hline b1 & b & 1 & 521.9996 & 522 & 0.00044 & 0.842913 \\
\hline 125 & C & 125 & 14610.91 & 14611.03 & 0.11725 & 8.024825 \\
\hline a136 & a & 136 & 15791.53 & 15791.61 & 0.083897 & 5.312784 \\
\hline$a+141$ & $a+$ & 141 & 16309.79 & 16309.82 & 0.025803 & 1.582054 \\
\hline a147 & a & 147 & 16957.1 & 16957.15 & 0.056512 & 3.332646 \\
\hline a157 & a & 157 & 18074.65 & 18074.79 & 0.141679 & 7.838548 \\
\hline b161 & b & 161 & 18578.91 & 18579.03 & 0.12596 & 6.779732 \\
\hline a161 & a & 161 & 18550.91 & 18551.09 & 0.173853 & 9.371669 \\
\hline a166 & a & 166 & 19233.34 & 19233.47 & 0.132103 & 6.868439 \\
\hline$x 8$ & $x$ & 8 & 1555.582 & 1555.572 & -0.00963 & -6.19382 \\
\hline$y-59$ & $y^{-}$ & 59 & 7183.493 & 7183.535 & 0.04282 & 5.960893 \\
\hline y69 & y & 69 & 8338.11 & 8338.137 & 0.02785 & 3.340086 \\
\hline z78 & $z$ & 78 & 9513.669 & 9513.708 & 0.038193 & 4.014539 \\
\hline x119 & $x$ & 119 & 14215.81 & 14215.91 & 0.100662 & 7.080992 \\
\hline$y-122$ & $y-$ & 122 & 14475.93 & 14476.06 & 0.129608 & 8.953347 \\
\hline$y-123$ & $y-$ & 123 & 14590.96 & 14591.03 & 0.072151 & 4.944914 \\
\hline$x+126$ & $x+$ & 126 & 14930.17 & 14930.27 & 0.092335 & 6.184454 \\
\hline x133 & $x$ & 133 & 15835.58 & 15835.67 & 0.086975 & 5.492377 \\
\hline$x+135$ & $x+$ & 135 & 16050.72 & 16050.84 & 0.111136 & 6.924047 \\
\hline$x+136$ & $x+$ & 136 & 16147.78 & 16147.89 & 0.114424 & 7.086051 \\
\hline$y-139$ & $y-$ & 139 & 16526.91 & 16527.01 & 0.097084 & 5.874299 \\
\hline y141 & $y$ & 141 & 16742.02 & 16742.14 & 0.122327 & 7.306586 \\
\hline$x 142$ & $x$ & 142 & 16915.07 & 16915.08 & 0.0185 & 1.0937 \\
\hline$x 144$ & $x$ & 144 & 17166.17 & 17166.32 & 0.149082 & 8.684641 \\
\hline$x 147$ & $x$ & 147 & 17520.39 & 17520.56 & 0.165546 & 9.44876 \\
\hline x153 & $x$ & 153 & 18133.74 & 18133.84 & 0.106009 & 5.845954 \\
\hline z156 & $z$ & 156 & 18375.92 & 18375.96 & 0.035883 & 1.952718 \\
\hline$x+158$ & $x+$ & 158 & 18578.96 & 18579.03 & 0.071425 & 3.844401 \\
\hline$y-158$ & $y-$ & 158 & 18550.97 & 18551.09 & 0.119318 & 6.431904 \\
\hline y160 & y & 160 & 18680.03 & 18680.13 & 0.094915 & 5.081094 \\
\hline z165 & $z$ & 165 & 19202.4 & 19202.43 & 0.030944 & 1.611465 \\
\hline x165 & $x$ & 165 & 19244.4 & 19244.56 & 0.159379 & 8.281839 \\
\hline
\end{tabular}


Table S1D. Summary of UVPD mass spectrum for G12C•GDP complex: fragment ions incorporating mass shift of +463.9859 Da (GDP)

\begin{tabular}{|c|c|c|c|c|c|c|}
\hline Ion & $\begin{array}{l}\text { Ion } \\
\text { Type }\end{array}$ & Residue & $\begin{array}{l}\text { Theoretical } \\
\text { Mass }\end{array}$ & $\begin{array}{l}\text { Experimental } \\
\text { Mass }\end{array}$ & $\Delta$ mass & $\begin{array}{l}\text { ppm } \\
\text { error }\end{array}$ \\
\hline c11 & c & 11 & 1657.633 & 1657.623 & -0.01061 & -6.40069 \\
\hline c75 & C & 75 & 8894.08 & 8894 & -0.07984 & -8.97676 \\
\hline$a+136$ & $a+$ & 136 & 15791.55 & 15791.61 & 0.068272 & 4.323324 \\
\hline c136 & C & 136 & 15835.56 & 15835.67 & 0.111525 & 7.042694 \\
\hline a138 & a & 138 & 16040.63 & 16040.79 & 0.152126 & 9.48379 \\
\hline c144 & c & 144 & 16742.01 & 16742.14 & 0.131622 & 7.861781 \\
\hline c145 & c & 145 & 16843.06 & 16843.08 & 0.026325 & 1.562959 \\
\hline$a+146$ & $a+$ & 146 & 16886.08 & 16886.23 & 0.156122 & 9.245604 \\
\hline$a+147$ & $a+$ & 147 & 16957.11 & 16957.15 & 0.040887 & 2.411199 \\
\hline b148 & $b$ & 148 & 17112.19 & 17112.29 & 0.09165 & 5.35583 \\
\hline c153 & c & 153 & 17670.52 & 17670.58 & 0.064008 & 3.622305 \\
\hline$a+157$ & $a+$ & 157 & 18074.66 & 18074.79 & 0.126054 & 6.97407 \\
\hline$a+161$ & $a+$ & 161 & 18550.93 & 18551.09 & 0.158228 & 8.529383 \\
\hline$a+166$ & $a+$ & 166 & 19233.35 & 19233.47 & 0.116478 & 6.056041 \\
\hline z47 & $z$ & 47 & 5912.864 & 5912.878 & 0.013717 & 2.319857 \\
\hline$x+48$ & $x+$ & 48 & 6042.902 & 6042.95 & 0.048097 & 7.95925 \\
\hline y68 & $y$ & 68 & 8211.022 & 8211.102 & 0.079488 & 9.680646 \\
\hline$y-69$ & $y-$ & 69 & 8338.11 & 8338.137 & 0.027875 & 3.343088 \\
\hline$x+71$ & $x+$ & 71 & 8607.247 & 8607.24 & -0.00751 & -0.87218 \\
\hline$x+84$ & $x+$ & 84 & 10264.01 & 10264.01 & 0.002772 & 0.270067 \\
\hline y121 & $y$ & 121 & 14420.92 & 14421.01 & 0.08528 & 5.913629 \\
\hline$x+122$ & $x+$ & 122 & 14504.93 & 14505.01 & 0.075684 & 5.217809 \\
\hline y125 & $y$ & 125 & 14805.13 & 14805.19 & 0.062317 & 4.20915 \\
\hline$x 126$ & $x$ & 126 & 14930.17 & 14930.27 & 0.09236 & 6.186131 \\
\hline$x+127$ & $x+$ & 127 & 15059.24 & 15059.32 & 0.076108 & 5.053905 \\
\hline z128 & $z$ & 128 & 15144.33 & 15144.4 & 0.073673 & 4.864725 \\
\hline x135 & $x$ & 135 & 16050.72 & 16050.84 & 0.111161 & 6.925607 \\
\hline y136 & y & 136 & 16121.8 & 16121.88 & 0.083948 & 5.207112 \\
\hline$x 136$ & $x$ & 136 & 16147.78 & 16147.89 & 0.114449 & 7.087601 \\
\hline z140 & $z$ & 140 & 16627.94 & 16628.1 & 0.163616 & 9.839825 \\
\hline$y-141$ & $y-$ & 141 & 16742.02 & 16742.14 & 0.122352 & 7.308081 \\
\hline y148 & $y$ & 148 & 17623.48 & 17623.5 & 0.018127 & 1.028571 \\
\hline z148 & $z$ & 148 & 17607.46 & 17607.51 & 0.051589 & 2.929951 \\
\hline z150 & $z$ & 150 & 17821.59 & 17821.7 & 0.103443 & 5.804363 \\
\hline z151 & $z$ & 151 & 17934.68 & 17934.69 & 0.007176 & 0.400119 \\
\hline z152 & $z$ & 152 & 18005.72 & 18005.8 & 0.080906 & 4.493351 \\
\hline$x+154$ & $x+$ & 154 & 18263.85 & 18263.95 & 0.105531 & 5.778134 \\
\hline z155 & $z$ & 155 & 18277.86 & 18277.98 & 0.11727 & 6.415958 \\
\hline
\end{tabular}




$\begin{array}{llllrrr}\mathrm{y} 157 & \mathrm{y} & 157 & 18449.97 & 18450.1 & 0.128996 & 6.991663 \\ \mathrm{x} 158 & \mathrm{x} & 158 & 18578.96 & 18579.03 & 0.07145 & 3.845748 \\ \mathrm{y}-160 & \mathrm{y}- & 160 & 18680.03 & 18680.13 & 0.09494 & 5.082434 \\ \mathrm{x}+164 & \mathrm{x}+ & 164 & 19118.32 & 19118.42 & 0.105413 & 5.513716\end{array}$


Table S2A. Summary of UVPD mass spectrum for G12C•GDPnP complex: apo fragment ions

Column "Ions" is the fragment ion identity $(a, b, c, x, y, z)$ along with the number of residues in the fragment ion. A plus or minus sign indicates the fragment ion has one additional hydrogen atom or one less hydrogen atom than a conventional fragment ion. Column "Ion Type" is the type of fragment ion $(a, b, c, x, y, z)$. A plus sign indicates a fragment ion $(a, x)$ with an additional hydrogen atom; a minus sign indicates a fragment ion $(y)$ that is missing one hydrogen atom (the addition of one hydrogen atom for $a / x$ ions is not uncommon in UVPD mass spectra of intact proteins); Column "Residue" is the number of residues in the fragment ion. Column "Theoretical Mass" is the theoretical mass of the fragment ion; Column "Experimental Mass" is the experimentally measured mass of the fragment ion. Column "Delta mass" is the mass difference between the theoretical and experimental masses; and column "ppm error" is the mass error in ppm. The "apo" table (Table S2A) provides the fragment ions that do not retain the ligand. Tables S2B, S2C, and S2D provide the fragment ions that incorporate the ligand based on the mass shift corresponding to the retained ligand (with or without an additional hydrogen atom).

\begin{tabular}{|c|c|c|c|c|c|c|}
\hline Ion & $\frac{\text { lon }}{\text { Type }}$ & Residue & $\begin{array}{l}\text { Theoretical } \\
\text { Mass }\end{array}$ & $\begin{array}{l}\text { Experimental } \\
\text { Mass }\end{array}$ & $\Delta$ mass & $\begin{array}{l}\text { ppm } \\
\text { error }\end{array}$ \\
\hline$\overline{a 4}$ & $\bar{a}$ & 4 & 390.1573 & 390.1595 & $\overline{0.002197}$ & $\overline{5.631062}$ \\
\hline a55 & a & 55 & 6026.015 & 6026.037 & 0.021919 & 3.637395 \\
\hline a54 & a & 54 & 5910.989 & 5911.015 & 0.026032 & 4.404001 \\
\hline a53 & a & 53 & 5797.904 & 5797.936 & 0.031113 & 5.366249 \\
\hline a51 & a & 51 & 5581.811 & 5581.843 & 0.03175 & 5.688118 \\
\hline a47 & a & 47 & 5179.673 & 5179.699 & 0.026827 & 5.179285 \\
\hline a45 & $\mathrm{a}$ & 45 & 4967.52 & 4967.541 & 0.020483 & 4.123385 \\
\hline a42 & a & 42 & 4612.298 & 4612.324 & 0.025514 & 5.531733 \\
\hline a41 & a & 41 & 4456.197 & 4456.218 & 0.020864 & 4.682019 \\
\hline a39 & a & 39 & 4206.102 & 4206.085 & -0.01682 & -3.99848 \\
\hline a38 & a & 38 & 4091.075 & 4091.093 & 0.017919 & 4.380023 \\
\hline a37 & a & 37 & 3962.032 & 3962.049 & 0.017052 & 4.303852 \\
\hline a56 & a & 56 & 6139.1 & 6139.108 & 0.00805 & 1.311267 \\
\hline a36 & a & 36 & 3848.948 & 3848.965 & 0.017372 & 4.513441 \\
\hline a30 & a & 30 & 3128.688 & 3128.706 & 0.01826 & 5.836313 \\
\hline a29 & a & 29 & 3029.619 & 3029.638 & 0.018921 & 6.245339 \\
\hline a28 & a & 28 & 2882.551 & 2882.568 & 0.016653 & 5.777174 \\
\hline a27 & a & 27 & 2745.492 & 2745.508 & 0.016311 & 5.941011 \\
\hline a25 & a & 25 & 2503.391 & 2503.406 & 0.015526 & 6.201989 \\
\hline a24 & a & 24 & 2390.307 & 2390.315 & 0.007986 & 3.340994 \\
\hline a20 & a & 20 & 1935.032 & 1935.044 & 0.011905 & 6.152353 \\
\hline a19 & a & 19 & 1821.948 & 1821.959 & 0.011162 & 6.12641 \\
\hline a17 & a & 17 & 1663.879 & 1663.89 & 0.010931 & 6.569589 \\
\hline a16 & a & 16 & 1535.784 & 1535.792 & 0.008309 & 5.410266 \\
\hline
\end{tabular}




\begin{tabular}{|c|c|c|c|c|c|c|}
\hline a32 & $a$ & 32 & 3372.757 & 3372.775 & 0.01815 & 5.381354 \\
\hline a15 & a & 15 & 1478.763 & 1478.772 & 0.009871 & 6.675176 \\
\hline a57 & $a$ & 57 & 6252.184 & 6252.21 & 0.026044 & 4.165585 \\
\hline a60 & a & 60 & 6539.295 & 6539.311 & 0.015629 & 2.390013 \\
\hline a101 & $a$ & 101 & 11433.53 & 11433.58 & 0.052619 & 4.602167 \\
\hline a97 & $a$ & 97 & 10907.24 & 10907.24 & -0.00012 & -0.01064 \\
\hline a96 & a & 96 & 10744.18 & 10744.22 & 0.044486 & 4.140475 \\
\hline a92 & a & 92 & 10241.95 & 10241.99 & 0.041738 & 4.075201 \\
\hline a91 & $a$ & 91 & 10112.91 & 10112.94 & 0.037139 & 3.672436 \\
\hline a90 & $a$ & 90 & 9965.838 & 9965.885 & 0.046833 & 4.699354 \\
\hline a88 & a & 88 & 9750.711 & 9750.763 & 0.051752 & 5.30751 \\
\hline a87 & a & 87 & 9649.664 & 9649.747 & 0.082944 & 8.595533 \\
\hline a86 & a & 86 & 9535.621 & 9535.667 & 0.046537 & 4.880332 \\
\hline a85 & a & 85 & 9421.578 & 9421.623 & 0.044927 & 4.768522 \\
\hline a83 & a & 83 & 9237.457 & 9237.491 & 0.034368 & 3.720505 \\
\hline a58 & a & 58 & 6367.211 & 6367.223 & 0.01204 & 1.890938 \\
\hline a81 & a & 81 & 8991.32 & 8991.399 & 0.078658 & 8.748215 \\
\hline a79 & a & 79 & 8775.227 & 8775.263 & 0.036044 & 4.107472 \\
\hline a78 & a & 78 & 8628.158 & 8628.194 & 0.035973 & 4.169256 \\
\hline a77 & a & 77 & 8571.137 & 8571.193 & 0.05609 & 6.544056 \\
\hline a75 & a & 75 & 8385.073 & 8385.116 & 0.042869 & 5.112538 \\
\hline a73 & a & 73 & 8127.924 & 8127.949 & 0.024986 & 3.074094 \\
\hline a72 & a & 72 & 7996.883 & 7996.917 & 0.033249 & 4.157745 \\
\hline a70 & $a$ & 70 & 7705.762 & 7705.79 & 0.028206 & 3.660378 \\
\hline a69 & $a$ & 69 & 7590.735 & 7590.768 & 0.033662 & 4.434617 \\
\hline a64 & a & 64 & 6982.461 & 6982.496 & 0.035956 & 5.149474 \\
\hline a63 & a & 63 & 6853.418 & 6853.454 & 0.035757 & 5.217397 \\
\hline a62 & a & 62 & 6724.375 & 6724.408 & 0.03256 & 4.842085 \\
\hline a80 & a & 80 & 8888.311 & 8888.354 & 0.043415 & 4.884505 \\
\hline a14 & $a$ & 14 & 1379.694 & 1379.705 & 0.010777 & 7.811152 \\
\hline a59 & a & 59 & 6468.258 & 6468.281 & 0.023198 & 3.586437 \\
\hline a11 & a & 11 & 1148.626 & 1148.634 & 0.007248 & 6.310146 \\
\hline a8 & a & 8 & 893.4681 & 893.4739 & 0.005799 & 6.490439 \\
\hline a13 & a & 13 & 1322.673 & 1322.681 & 0.007945 & 6.006777 \\
\hline a5 & a & 5 & 553.2206 & 553.224 & 0.003412 & 6.167521 \\
\hline a9 & a & 9 & 992.5365 & 992.543 & 0.00648 & 6.528727 \\
\hline a10 & a & 10 & 1091.605 & 1091.612 & 0.007101 & 6.505101 \\
\hline$a+27$ & $a+$ & 27 & 2746.5 & 2746.514 & 0.013613 & 4.956479 \\
\hline$a+43$ & $a+$ & 43 & 4741.401 & 4741.425 & 0.024536 & 5.174835 \\
\hline$a+4$ & $a+$ & 4 & 391.1651 & 391.1678 & 0.002703 & 6.910043 \\
\hline$a+25$ & $a+$ & 25 & 2504.398 & 2504.411 & 0.012584 & 5.024747 \\
\hline$a+24$ & $a+$ & 24 & 2391.314 & 2391.325 & 0.01046 & 4.37415 \\
\hline$a+7$ & $a+$ & 7 & 795.4075 & 795.413 & 0.005531 & 6.953628 \\
\hline
\end{tabular}




\begin{tabular}{|c|c|c|c|c|c|c|}
\hline$a+93$ & $a+$ & 93 & 10357.98 & 10358.02 & 0.037298 & 3.600891 \\
\hline$a+89$ & $a+$ & 89 & 9879.814 & 9879.844 & 0.029778 & 3.014021 \\
\hline$a+65$ & $a+$ & 65 & 7146.532 & 7146.548 & 0.015874 & 2.221213 \\
\hline$a+30$ & $a+$ & 30 & 3129.696 & 3129.71 & 0.014585 & 4.660187 \\
\hline$a+44$ & $a+$ & 44 & 4869.459 & 4869.484 & 0.024061 & 4.941199 \\
\hline$a+71$ & $a+$ & 71 & 7834.828 & 7834.859 & 0.030649 & 3.911888 \\
\hline$a+31$ & $a+$ & 31 & 3244.723 & 3244.742 & 0.018994 & 5.853803 \\
\hline$a+32$ & $a+$ & 32 & 3373.765 & 3373.781 & 0.016175 & 4.794337 \\
\hline$a+11$ & $a+$ & 11 & 1149.634 & 1149.641 & 0.007113 & 6.187158 \\
\hline$a+82$ & $a+$ & 82 & 9091.396 & 9091.447 & 0.051251 & 5.637304 \\
\hline$a+40$ & $a+$ & 40 & 4294.142 & 4294.159 & 0.017775 & 4.139353 \\
\hline$a+34$ & $a+$ & 34 & 3651.855 & 3651.874 & 0.018848 & 5.161203 \\
\hline$a+74$ & $a+$ & 74 & 8285.033 & 8285.073 & 0.04061 & 4.901606 \\
\hline$a+35$ & $a+$ & 35 & 3748.908 & 3748.924 & 0.015967 & 4.259098 \\
\hline$a+6$ & $a+$ & 6 & 682.3234 & 682.3279 & 0.004447 & 6.51739 \\
\hline$a+28$ & $a+$ & 28 & 2883.559 & 2883.575 & 0.015725 & 5.45332 \\
\hline$a+23$ & $a+$ & 23 & 2278.23 & 2278.239 & 0.008537 & 3.747193 \\
\hline$a+22$ & $a+$ & 22 & 2150.172 & 2150.184 & 0.011989 & 5.575819 \\
\hline$a+143$ & $a+$ & 143 & 16104.97 & 16104.87 & -0.10091 & -6.2659 \\
\hline$a+16$ & $a+$ & 16 & 1536.792 & 1536.799 & 0.006832 & 4.445604 \\
\hline$a+9$ & $a+$ & 9 & 993.5443 & 993.5506 & 0.006346 & 6.387202 \\
\hline$a+51$ & $a+$ & 51 & 5582.819 & 5582.844 & 0.025107 & 4.497185 \\
\hline$a+13$ & $a+$ & 13 & 1323.68 & 1323.684 & 0.003171 & 2.395569 \\
\hline$a+17$ & $a+$ & 17 & 1664.887 & 1664.896 & 0.00904 & 5.429779 \\
\hline$a+50$ & $a+$ & 50 & 5481.771 & 5481.793 & 0.022006 & 4.01439 \\
\hline$a+38$ & $a+$ & 38 & 4092.083 & 4092.101 & 0.018321 & 4.477175 \\
\hline$a+49$ & $a+$ & 49 & 5352.729 & 5352.75 & 0.021017 & 3.926403 \\
\hline$a+14$ & $a+$ & 14 & 1380.702 & 1380.71 & 0.008201 & 5.939709 \\
\hline$a+18$ & $a+$ & 18 & 1751.919 & 1751.928 & 0.009602 & 5.480829 \\
\hline$a+8$ & $a+$ & 8 & 894.4759 & 894.4823 & 0.006396 & 7.15052 \\
\hline$a+19$ & $a+$ & 19 & 1822.956 & 1822.965 & 0.008962 & 4.916174 \\
\hline$a+12$ & $a+$ & 12 & 1220.671 & 1220.678 & 0.006746 & 5.526441 \\
\hline$a+48$ & $a+$ & 48 & 5295.707 & 5295.731 & 0.024166 & 4.563313 \\
\hline$a+20$ & $a+$ & 20 & 1936.04 & 1936.049 & 0.009282 & 4.794306 \\
\hline$a+94$ & $a+$ & 94 & 10471.07 & 10471.11 & 0.042054 & 4.016206 \\
\hline$a+46$ & $a+$ & 46 & 5067.596 & 5067.619 & 0.022739 & 4.487131 \\
\hline$a+61$ & $a+$ & 61 & 6597.325 & 6597.347 & 0.021988 & 3.332861 \\
\hline$a+21$ & $a+$ & 21 & 2037.088 & 2037.097 & 0.009219 & 4.525563 \\
\hline$a+15$ & $a+$ & 15 & 1479.77 & 1479.777 & 0.006929 & 4.682462 \\
\hline b6 & $b$ & 6 & 709.3105 & 709.3152 & 0.004662 & 6.57258 \\
\hline b8 & b & 8 & 921.463 & 921.469 & 0.006062 & 6.578669 \\
\hline b9 & $b$ & 9 & 1020.531 & 1020.537 & 0.006011 & 5.890069 \\
\hline b49 & $b$ & 49 & 5379.716 & 5379.744 & 0.028171 & 5.23652 \\
\hline
\end{tabular}




\begin{tabular}{|c|c|c|c|c|c|c|}
\hline b52 & $b$ & 52 & 5712.815 & 5712.844 & 0.028583 & 5.003312 \\
\hline b7 & b & 7 & 822.3946 & 822.4 & 0.005441 & 6.616046 \\
\hline b43 & $b$ & 43 & 4768.388 & 4768.417 & 0.029402 & 6.166025 \\
\hline b5 & b & 5 & 581.2156 & 581.2192 & 0.003614 & 6.218003 \\
\hline b80 & $b$ & 80 & 8916.306 & 8916.357 & 0.051137 & 5.735223 \\
\hline b21 & b & 21 & 2064.075 & 2064.087 & 0.012768 & 6.185823 \\
\hline b20 & $b$ & 20 & 1963.027 & 1963.04 & 0.012718 & 6.47877 \\
\hline b4 & $b$ & 4 & 418.1522 & 418.1546 & 0.002369 & 5.665401 \\
\hline b19 & $b$ & 19 & 1849.943 & 1849.955 & 0.011695 & 6.321816 \\
\hline b12 & $b$ & 12 & 1247.658 & 1247.666 & 0.007937 & 6.361517 \\
\hline b23 & $b$ & 23 & 2305.217 & 2305.23 & 0.012706 & 5.511845 \\
\hline b17 & $b$ & 17 & 1691.874 & 1691.884 & 0.010353 & 6.119251 \\
\hline b16 & $b$ & 16 & 1563.779 & 1563.787 & 0.007901 & 5.052505 \\
\hline b15 & $b$ & 15 & 1506.757 & 1506.768 & 0.01044 & 6.928786 \\
\hline b13 & $b$ & 13 & 1350.668 & 1350.676 & 0.008513 & 6.302809 \\
\hline b14 & $b$ & 14 & 1407.689 & 1407.697 & 0.008171 & 5.804549 \\
\hline b144 & $b$ & 144 & 16261 & 16260.86 & -0.13938 & -8.57149 \\
\hline b10 & $b$ & 10 & 1119.6 & 1119.607 & 0.007669 & 6.849769 \\
\hline b25 & $b$ & 25 & 2531.385 & 2531.397 & 0.011334 & 4.47739 \\
\hline b11 & $b$ & 11 & 1176.621 & 1176.629 & 0.007572 & 6.435376 \\
\hline b31 & b & 31 & 3271.71 & 3271.73 & 0.020161 & 6.162222 \\
\hline b32 & $b$ & 32 & 3400.752 & 3400.775 & 0.023226 & 6.829665 \\
\hline b34 & $b$ & 34 & 3678.843 & 3678.864 & 0.021823 & 5.932029 \\
\hline c5 & c & 5 & 598.2419 & 598.2458 & 0.003986 & 6.662857 \\
\hline c81 & c & 81 & 9036.341 & 9036.41 & 0.06843 & 7.572755 \\
\hline c72 & c & 72 & 8041.905 & 8041.895 & -0.00994 & -1.23578 \\
\hline c61 & c & 61 & 6641.338 & 6641.313 & -0.0248 & -3.73434 \\
\hline c59 & c & 59 & 6513.279 & 6513.262 & -0.01701 & -2.61205 \\
\hline c4 & c & 4 & 435.1785 & 435.1812 & 0.00271 & 6.227329 \\
\hline$c 64$ & c & 64 & 7027.482 & 7027.449 & -0.03282 & -4.67009 \\
\hline c13 & c & 13 & 1367.694 & 1367.703 & 0.008702 & 6.362535 \\
\hline c17 & c & 17 & 1708.9 & 1708.907 & 0.007002 & 4.097372 \\
\hline c34 & c & 34 & 3695.869 & 3695.838 & -0.03092 & -8.36583 \\
\hline c33 & c & 33 & 3580.842 & 3580.808 & -0.03376 & -9.42907 \\
\hline c31 & c & 31 & 3288.736 & 3288.753 & 0.017299 & 5.260076 \\
\hline c29 & c & 29 & 3074.641 & 3074.656 & 0.015481 & 5.03506 \\
\hline c26 & c & 26 & 2676.47 & 2676.486 & 0.015809 & 5.90666 \\
\hline$c 25$ & c & 25 & 2548.412 & 2548.428 & 0.01604 & 6.294116 \\
\hline c11 & c & 11 & 1193.648 & 1193.657 & 0.009104 & 7.627042 \\
\hline$c 23$ & c & 23 & 2322.244 & 2322.257 & 0.013749 & 5.920567 \\
\hline c20 & c & 20 & 1980.053 & 1980.066 & 0.012663 & 6.395282 \\
\hline c16 & c & 16 & 1580.805 & 1580.816 & 0.010335 & 6.537807 \\
\hline c14 & C & 14 & 1424.715 & 1424.722 & 0.006896 & 4.840265 \\
\hline
\end{tabular}




\begin{tabular}{|c|c|c|c|c|c|c|}
\hline c58 & c & 58 & 6412.232 & 6412.229 & -0.00243 & -0.37834 \\
\hline c36 & c & 36 & 3893.969 & 3893.99 & 0.020571 & 5.282784 \\
\hline c37 & c & 37 & 4007.053 & 4007.025 & -0.02841 & -7.08925 \\
\hline$c 27$ & c & 27 & 2790.513 & 2790.528 & 0.014871 & 5.329127 \\
\hline c38 & c & 38 & 4136.096 & 4136.115 & 0.018921 & 4.574604 \\
\hline$c 8$ & c & 8 & 938.4893 & 938.4958 & 0.006495 & 6.920697 \\
\hline c50 & c & 50 & 5525.785 & 5525.792 & 0.007438 & 1.346053 \\
\hline$c 9$ & c & 9 & 1037.558 & 1037.565 & 0.007177 & 6.917206 \\
\hline$c 45$ & c & 45 & 5012.541 & 5012.578 & 0.036255 & 7.232858 \\
\hline c10 & c & 10 & 1136.626 & 1136.633 & 0.00737 & 6.484102 \\
\hline c44 & c & 44 & 4913.473 & 4913.489 & 0.016408 & 3.33939 \\
\hline$c 43$ & c & 43 & 4785.414 & 4785.382 & -0.03203 & -6.69263 \\
\hline c41 & c & 41 & 4501.218 & 4501.254 & 0.035678 & 7.926299 \\
\hline c40 & c & 40 & 4338.155 & 4338.179 & 0.023916 & 5.512943 \\
\hline c39 & C & 39 & 4251.123 & 4251.143 & 0.020302 & 4.775679 \\
\hline c42 & c & 42 & 4657.319 & 4657.351 & 0.032131 & 6.899033 \\
\hline$x 6$ & $x$ & 6 & 850.4773 & 850.4829 & 0.005577 & 6.557494 \\
\hline$x 5$ & $x$ & 5 & 694.3762 & 694.3807 & 0.004514 & 6.500799 \\
\hline x31 & $x$ & 31 & 3700.969 & 3700.986 & 0.017563 & 4.745514 \\
\hline x11 & $x$ & 11 & 1460.858 & 1460.866 & 0.008831 & 6.045079 \\
\hline x9 & $x$ & 9 & 1248.705 & 1248.713 & 0.007858 & 6.292919 \\
\hline$x 7$ & $x$ & 7 & 963.5614 & 963.5676 & 0.006172 & 6.405404 \\
\hline x38 & $x$ & 38 & 4463.335 & 4463.374 & 0.039388 & 8.824792 \\
\hline$x 37$ & $x$ & 37 & 4348.308 & 4348.332 & 0.023726 & 5.456375 \\
\hline$x 13$ & $x$ & 13 & 1724.969 & 1724.98 & 0.011882 & 6.888241 \\
\hline x4 & $x$ & 4 & 566.2813 & 566.2848 & 0.003527 & 6.228354 \\
\hline$x 10$ & $x$ & 10 & 1347.773 & 1347.782 & 0.00854 & 6.336376 \\
\hline$x 20$ & $x$ & 20 & 2457.276 & 2457.291 & 0.014213 & 5.784046 \\
\hline$x 28$ & $x$ & 28 & 3343.764 & 3343.78 & 0.01625 & 4.859793 \\
\hline$x 48$ & $x$ & 48 & 5577.908 & 5577.947 & 0.038685 & 6.935396 \\
\hline$x 3$ & $x$ & 3 & 429.2223 & 429.2247 & 0.002409 & 5.612476 \\
\hline$\times 29$ & $x$ & 29 & 3490.832 & 3490.851 & 0.019366 & 5.547675 \\
\hline$x 45$ & $x$ & 45 & 5233.727 & 5233.765 & 0.037979 & 7.256588 \\
\hline$x+12$ & $x+$ & 12 & 1562.913 & 1562.919 & 0.005985 & 3.829367 \\
\hline$x+31$ & $x+$ & 31 & 3701.977 & 3701.995 & 0.018331 & 4.95167 \\
\hline$x+32$ & $x+$ & 32 & 3758.998 & 3759.013 & 0.014689 & 3.907682 \\
\hline$x+14$ & $x+$ & 14 & 1873.045 & 1873.051 & 0.006692 & 3.572775 \\
\hline$x+16$ & $x+$ & 16 & 2059.109 & 2059.118 & 0.008926 & 4.334869 \\
\hline$x+34$ & $x+$ & 34 & 4009.094 & 4009.114 & 0.020253 & 5.051757 \\
\hline$x+27$ & $x+$ & 27 & 3231.687 & 3231.717 & 0.029633 & 9.169503 \\
\hline$x+23$ & $x+$ & 23 & 2843.528 & 2843.536 & 0.008156 & 2.868257 \\
\hline$x+15$ & $x+$ & 15 & 1944.082 & 1944.09 & 0.008156 & 4.19528 \\
\hline$x+21$ & $x+$ & 21 & 2614.385 & 2614.397 & 0.011967 & 4.577354 \\
\hline
\end{tabular}




\begin{tabular}{|c|c|c|c|c|c|c|}
\hline$x+44$ & $x+$ & 44 & 5135.667 & 5135.704 & 0.037669 & 7.334777 \\
\hline$x+45$ & $x+$ & 45 & 5234.735 & 5234.765 & 0.029845 & 5.701333 \\
\hline$x+35$ & $x+$ & 35 & 4165.195 & 4165.201 & 0.0067 & 1.608561 \\
\hline$x+52$ & $x+$ & 52 & 6007.089 & 6007.126 & 0.036973 & 6.15489 \\
\hline$x+43$ & $x+$ & 43 & 5020.64 & 5020.671 & 0.031151 & 6.204582 \\
\hline$x+55$ & $x+$ & 55 & 6306.248 & 6306.284 & 0.035505 & 5.630125 \\
\hline$x+41$ & $x+$ & 41 & 4791.497 & 4791.517 & 0.020238 & 4.223725 \\
\hline$x+42$ & $x+$ & 42 & 4919.592 & 4919.616 & 0.024399 & 4.959551 \\
\hline$x+40$ & $x+$ & 40 & 4663.438 & 4663.459 & 0.020346 & 4.362868 \\
\hline$x+39$ & $x+$ & 39 & 4592.401 & 4592.423 & 0.021689 & 4.722795 \\
\hline$x+62$ & $x+$ & 62 & 7059.658 & 7059.687 & 0.029666 & 4.202182 \\
\hline$x+48$ & $x+$ & 48 & 5578.916 & 5578.949 & 0.033604 & 6.023387 \\
\hline y52 & $y$ & 52 & 5980.102 & 5980.131 & 0.029474 & 4.928679 \\
\hline y23 & y & 23 & 2816.541 & 2816.557 & 0.016573 & 5.884168 \\
\hline y61 & y & 61 & 6917.644 & 6917.678 & 0.03437 & 4.968455 \\
\hline y28 & y & 28 & 3317.784 & 3317.798 & 0.013582 & 4.093696 \\
\hline y26 & y & 26 & 3075.658 & 3075.673 & 0.014791 & 4.809053 \\
\hline y35 & y & 35 & 4138.208 & 4138.229 & 0.021622 & 5.224968 \\
\hline y21 & $y$ & 21 & 2587.398 & 2587.413 & 0.014926 & 5.768729 \\
\hline y29 & y & 29 & 3464.853 & 3464.876 & 0.023297 & 6.723807 \\
\hline y31 & y & 31 & 3674.99 & 3674.999 & 0.009279 & 2.524905 \\
\hline y43 & y & 43 & 4993.653 & 4993.678 & 0.02538 & 5.082452 \\
\hline y41 & $y$ & 41 & 4764.51 & 4764.518 & 0.00841 & 1.765134 \\
\hline y40 & $y$ & 40 & 4636.451 & 4636.471 & 0.019244 & 4.150588 \\
\hline y32 & y & 32 & 3732.011 & 3732.031 & 0.019483 & 5.22051 \\
\hline y36 & y & 36 & 4209.245 & 4209.269 & 0.024411 & 5.799378 \\
\hline y47 & y & 47 & 5464.897 & 5464.927 & 0.030157 & 5.518311 \\
\hline y168 & y & 168 & 19148.59 & 19148.44 & -0.14862 & -7.76167 \\
\hline y12 & y & 12 & 1535.926 & 1535.936 & 0.009739 & 6.3408 \\
\hline y20 & y & 20 & 2431.297 & 2431.316 & 0.019112 & 7.860824 \\
\hline y11 & y & 11 & 1434.878 & 1434.888 & 0.009336 & 6.506475 \\
\hline y10 & y & 10 & 1321.794 & 1321.803 & 0.008801 & 6.658374 \\
\hline y9 & y & 9 & 1222.726 & 1222.734 & 0.007997 & 6.540305 \\
\hline y8 & y & 8 & 1066.625 & 1066.632 & 0.007301 & 6.844957 \\
\hline y7 & y & 7 & 937.5821 & 937.5882 & 0.006067 & 6.4709 \\
\hline y6 & y & 6 & 824.4981 & 824.5035 & 0.005472 & 6.636765 \\
\hline y5 & y & 5 & 668.3969 & 668.4013 & 0.004348 & 6.505117 \\
\hline y4 & y & 4 & 540.302 & 540.3053 & 0.003299 & 6.105845 \\
\hline y3 & y & 3 & 403.2431 & 403.2453 & 0.002273 & 5.636799 \\
\hline y13 & y & 13 & 1698.989 & 1699.001 & 0.011655 & 6.859961 \\
\hline y19 & y & 19 & 2303.239 & 2303.226 & -0.01215 & -5.27562 \\
\hline y14 & y & 14 & 1846.058 & 1846.07 & 0.012581 & 6.815063 \\
\hline y15 & y & 15 & 1917.095 & 1917.108 & 0.013068 & 6.816564 \\
\hline
\end{tabular}




\begin{tabular}{|c|c|c|c|c|c|c|}
\hline y16 & y & 16 & 2032.122 & 2032.135 & 0.012862 & 6.329345 \\
\hline$y-30$ & $y-$ & 30 & 3560.898 & 3560.915 & 0.017106 & 4.803854 \\
\hline$y-3$ & $y-$ & 3 & 402.2352 & 402.2375 & 0.002255 & 5.606252 \\
\hline$y-40$ & $y-$ & 40 & 4635.443 & 4635.463 & 0.019533 & 4.213843 \\
\hline$y-22$ & $y-$ & 22 & 2687.438 & 2687.45 & 0.011936 & 4.441417 \\
\hline$y-42$ & $y-$ & 42 & 4891.597 & 4891.627 & 0.0303 & 6.194303 \\
\hline$y-18$ & $y-$ & 18 & 2245.209 & 2245.219 & 0.009321 & 4.15152 \\
\hline$y-26$ & $y-$ & 26 & 3074.65 & 3074.656 & 0.006211 & 2.020078 \\
\hline$y-41$ & $y-$ & 41 & 4763.502 & 4763.533 & 0.030426 & 6.387324 \\
\hline$y-39$ & $y-$ & 39 & 4564.406 & 4564.436 & 0.030048 & 6.583119 \\
\hline$y-28$ & $y-$ & 28 & 3316.777 & 3316.792 & 0.015107 & 4.554733 \\
\hline$y-21$ & $y-$ & 21 & 2586.39 & 2586.394 & 0.003172 & 1.226432 \\
\hline$y-37$ & $y-$ & 37 & 4321.321 & 4321.343 & 0.021802 & 5.045224 \\
\hline$y-56$ & $y-$ & 56 & 6377.322 & 6377.348 & 0.026247 & 4.115683 \\
\hline$y-36$ & $y-$ & 36 & 4208.237 & 4208.256 & 0.019558 & 4.64756 \\
\hline$y-29$ & $y-$ & 29 & 3463.845 & 3463.861 & 0.016277 & 4.699123 \\
\hline$y-60$ & $y-$ & 60 & 6817.567 & 6817.603 & 0.035729 & 5.24073 \\
\hline$y-33$ & $y-$ & 33 & 3894.067 & 3894.087 & 0.020009 & 5.138338 \\
\hline$y-32$ & $y-$ & 32 & 3731.003 & 3731.019 & 0.0162 & 4.342004 \\
\hline$y-25$ & $y-$ & 25 & 2973.602 & 2973.616 & 0.013608 & 4.576279 \\
\hline$y-24$ & $y-$ & 24 & 2886.57 & 2886.584 & 0.014308 & 4.956759 \\
\hline z50 & $z$ & 50 & 5746.047 & 5746.089 & 0.042288 & 7.359495 \\
\hline z3 & z & 3 & 387.2243 & 387.2265 & 0.002209 & 5.704704 \\
\hline$z 64$ & z & 64 & 7232.726 & 7232.772 & 0.045325 & 6.266655 \\
\hline z168 & z & 168 & 19132.57 & 19132.41 & -0.16181 & -8.4573 \\
\hline z6 & $z$ & 6 & 808.4793 & 808.485 & 0.005743 & 7.10346 \\
\hline z41 & $z$ & 41 & 4748.491 & 4748.524 & 0.032562 & 6.857336 \\
\hline z13 & z & 13 & 1682.971 & 1682.979 & 0.008935 & 5.309065 \\
\hline z7 & z & 7 & 921.5634 & 921.5699 & 0.006583 & 7.143296 \\
\hline z40 & z & 40 & 4620.433 & 4620.445 & 0.012163 & 2.632438 \\
\hline z8 & z & 8 & 1050.606 & 1050.613 & 0.007145 & 6.800837 \\
\hline z15 & z & 15 & 1901.076 & 1901.086 & 0.010105 & 5.315411 \\
\hline z35 & z & 35 & 4122.189 & 4122.206 & 0.016827 & 4.082055 \\
\hline z10 & z & 10 & 1305.775 & 1305.783 & 0.007669 & 5.873138 \\
\hline z11 & z & 11 & 1418.86 & 1418.865 & 0.00564 & 3.975024 \\
\hline z14 & z & 14 & 1830.039 & 1830.049 & 0.009617 & 5.255079 \\
\hline z32 & z & 32 & 3715.992 & 3716.003 & 0.010294 & 2.770189 \\
\hline$z 27$ & $z$ & 27 & 3188.682 & 3188.699 & 0.017944 & 5.627404 \\
\hline z169 & $z$ & 169 & 19263.61 & 19263.53 & -0.08441 & -4.38199 \\
\hline
\end{tabular}


Table S2B. Summary of UVPD mass spectrum for G12C•GDPnP complex: fragment ions incorporating mass shift of +543.9760 Da (GDPnp)

\begin{tabular}{|c|c|c|c|c|c|c|}
\hline & $\underline{\text { Ion }}$ & & Theoretical & Experimental & & $\mathrm{ppm}$ \\
\hline Ion & Type & Residue & Mass & Mass & $\Delta$ mass & error \\
\hline a125 & a & 125 & 14644.89 & 14644.98 & 0.089096 & 6.083761 \\
\hline a136 & a & 136 & 15870.53 & 15870.69 & 0.156067 & 9.833762 \\
\hline a140 & a & 140 & 16290.73 & 16290.78 & 0.04674 & 2.869116 \\
\hline b147 & $b$ & 147 & 17064.09 & 17064.13 & 0.041486 & 2.431187 \\
\hline$a+157$ & $a+$ & 157 & 18154.65 & 18154.74 & 0.082243 & 4.530131 \\
\hline a158 & $\mathrm{a}$ & 158 & 18316.71 & 18316.87 & 0.159795 & 8.724001 \\
\hline c163 & c & 163 & 18960.07 & 18960.14 & 0.064993 & 3.427887 \\
\hline a165 & a & 165 & 19184.24 & 19184.38 & 0.138587 & 7.224003 \\
\hline z16 & $z$ & 16 & 2560.079 & 2560.081 & 0.002356 & 0.920284 \\
\hline$x 47$ & $x$ & 47 & 6034.852 & 6034.911 & 0.05879 & 9.741747 \\
\hline y66 & y & 66 & 8035.843 & 8035.922 & 0.078562 & 9.776448 \\
\hline z79 & $z$ & 79 & 9723.71 & 9723.7 & -0.01023 & -1.05217 \\
\hline$y-80$ & $y-$ & 80 & 9885.789 & 9885.834 & 0.045031 & 4.555128 \\
\hline$x 87$ & $x$ & 87 & 10641.16 & 10641.18 & 0.021615 & 2.031264 \\
\hline$x+110$ & $x+$ & 110 & 13340.33 & 13340.34 & 0.015117 & 1.133178 \\
\hline$x+118$ & $x+$ & 118 & 14194.8 & 14194.81 & 0.008934 & 0.629383 \\
\hline z119 & $z$ & 119 & 14254.81 & 14254.84 & 0.030013 & 2.105465 \\
\hline$y-119$ & $y-$ & 119 & 14269.82 & 14269.85 & 0.038604 & 2.705293 \\
\hline$y-121$ & $y-$ & 121 & 14499.91 & 14499.94 & 0.033783 & 2.329879 \\
\hline$x+122$ & $x+$ & 122 & 14584.92 & 14584.97 & 0.044264 & 3.034913 \\
\hline$x+123$ & $x+$ & 123 & 14699.95 & 14700.02 & 0.068593 & 4.666204 \\
\hline$y-124$ & $y-$ & 124 & 14785.04 & 14785.04 & -0.00346 & -0.23415 \\
\hline$x 125$ & $x$ & 125 & 14911.09 & 14911.18 & 0.086058 & 5.771407 \\
\hline$x 126$ & $x$ & 126 & 15010.16 & 15010.21 & 0.044747 & 2.981114 \\
\hline$x+127$ & $x+$ & 127 & 15139.23 & 15139.29 & 0.059153 & 3.907264 \\
\hline$y-128$ & $y-$ & 128 & 15239.33 & 15239.34 & 0.01526 & 1.001358 \\
\hline x128 & $x$ & 128 & 15266.32 & 15266.34 & 0.021579 & 1.413504 \\
\hline z128 & $z$ & 128 & 15224.32 & 15224.41 & 0.093339 & 6.130915 \\
\hline$y-129$ & $y-$ & 129 & 15395.43 & 15395.45 & 0.022402 & 1.455109 \\
\hline x133 & $x$ & 133 & 15916.58 & 15916.59 & 0.010133 & 0.636632 \\
\hline y135 & y & 135 & 16104.74 & 16104.87 & 0.130983 & 8.133198 \\
\hline$y-136$ & $y-$ & 136 & 16200.78 & 16200.88 & 0.099232 & 6.125139 \\
\hline$y-137$ & $y^{-}$ & 137 & 16315.81 & 16315.86 & 0.055202 & 3.383347 \\
\hline$x+138$ & $x+$ & 138 & 16506.87 & 16506.9 & 0.031625 & 1.915868 \\
\hline$y-140$ & $y^{-}$ & 140 & 16722.94 & 16722.99 & 0.04783 & 2.860145 \\
\hline x141 & $x$ & 141 & 16849 & 16849.02 & 0.027486 & 1.631314 \\
\hline$y-144$ & $y-$ & 144 & 17220.18 & 17220.22 & 0.045986 & 2.670474 \\
\hline x149 & $x$ & 149 & 17842.53 & 17842.58 & 0.048491 & 2.717719 \\
\hline
\end{tabular}




$\begin{array}{llllllr}z 153 & z & 153 & 18172.74 & 18172.73 & -0.01105 & -0.608 \\ x+154 & x+ & 154 & 18343.84 & 18343.84 & -0.00322 & -0.17559 \\ \text { y154 } & y & 154 & 18316.85 & 18316.87 & 0.017805 & 0.972056 \\ \text { y157 } & y & 157 & 18529.96 & 18529.96 & -0.00368 & -0.19871 \\ \text { z158 } & z & 158 & 18616.95 & 18616.98 & 0.023554 & 1.265191 \\ \text { y-160 } & y- & 160 & 18760.02 & 18760.11 & 0.088727 & 4.72958 \\ \text { x161 } & x & 161 & 18886.08 & 18886.15 & 0.070825 & 3.750117 \\ \text { x163 } & x & 163 & 19084.22 & 19084.37 & 0.155829 & 8.165335 \\ \text { y169 } & y & 169 & 19823.61 & 19823.76 & 0.151291 & 7.63186\end{array}$


Table S2C. Summary of UVPD mass spectrum for G12C•GDPnP complex: fragment ions incorporating mass shift of +541.9604 Da (GDPnp)

\begin{tabular}{|c|c|c|c|c|c|c|}
\hline & $\underline{\text { Ion }}$ & & Theoretical & Experimental & & $\mathrm{ppm}$ \\
\hline Ion & Type & Residue & Mass & Mass & $\Delta$ mass & error \\
\hline b11 & $\mathrm{b}$ & 11 & 1718.582 & 1718.568 & -0.01411 & -8.20909 \\
\hline b16 & $b$ & 16 & 2105.739 & 2105.729 & -0.00999 & -4.74608 \\
\hline 111 & a & 111 & 13129.1 & 13129 & -0.09741 & -7.41978 \\
\hline b125 & $b$ & 125 & 14670.87 & 14671 & 0.129512 & 8.827836 \\
\hline c130 & c & 130 & 15259.19 & 15259.33 & 0.138803 & 9.096355 \\
\hline b142 & $b$ & 142 & 16560.83 & 16560.89 & 0.056166 & 3.391497 \\
\hline c144 & c & 144 & 16819.98 & 16820.06 & 0.078021 & 4.63859 \\
\hline c157 & c & 157 & 18196.65 & 18196.75 & 0.099834 & 5.486394 \\
\hline$a+160$ & $a+$ & 160 & 18529.83 & 18529.96 & 0.125673 & 6.782196 \\
\hline b161 & $b$ & 161 & 18655.89 & 18655.98 & 0.094099 & 5.04393 \\
\hline$a+162$ & $a+$ & 162 & 18785 & 18785.06 & 0.05796 & 3.085438 \\
\hline$x 70$ & $x$ & 70 & 8556.155 & 8556.189 & 0.033983 & 3.971761 \\
\hline$y-71$ & $y-$ & 71 & 8657.227 & 8657.194 & -0.03284 & -3.79371 \\
\hline$y-73$ & $y-$ & 73 & 8942.37 & 8942.384 & 0.013251 & 1.481825 \\
\hline$y-79$ & $y-$ & 79 & 9736.705 & 9736.733 & 0.027876 & 2.862984 \\
\hline z82 & $z$ & 82 & 10083.89 & 10083.94 & 0.05472 & 5.426477 \\
\hline z88 & $z$ & 88 & 10744.21 & 10744.22 & 0.009991 & 0.929896 \\
\hline$x+117$ & $x+$ & 117 & 79.7 & 79.72 & 0.017041 & 1.210322 \\
\hline z122 & $z$ & 122 & 14539.9 & 14539.93 & 0.026656 & 1.8333 \\
\hline y123 & $y$ & 123 & 14670.95 & 14671 & 0.049067 & 3.344501 \\
\hline$x+124$ & $x+$ & 124 & 14811.02 & 14811.07 & 0.051159 & 3.454115 \\
\hline$y-126$ & $y^{-}$ & 126 & 14981.16 & 14981.27 & 0.114332 & 7.631721 \\
\hline y127 & y & 127 & 15110.23 & 15110.26 & 0.02864 & 1.895405 \\
\hline$x+129$ & $x+$ & 129 & 15421.41 & 15421.44 & 0.026772 & 1.736026 \\
\hline$x+130$ & $x+$ & 130 & 15584.47 & 15584.51 & 0.033046 & 2.120442 \\
\hline$x+131$ & $x+$ & 131 & 15671.51 & 15671.55 & 0.048909 & 3.120885 \\
\hline$x+132$ & $x+$ & 132 & 15786.53 & 15786.56 & 0.028658 & 1.815343 \\
\hline y138 & y & 138 & 16477.86 & 16477.97 & 0.103651 & 6.290318 \\
\hline$x+139$ & $x+$ & 139 & 16633.89 & 16634 & 0.112057 & 6.736666 \\
\hline z140 & $z$ & 140 & 16705.91 & 16706.02 & 0.10269 & 6.146925 \\
\hline$y-141$ & $y^{-}$ & 141 & 16819.99 & 16820.06 & 0.068751 & 4.087459 \\
\hline y142 & y & 142 & 16968.07 & 16968.15 & 0.082359 & 4.853764 \\
\hline$x 142$ & $x$ & 142 & 16994.05 & 16994.17 & 0.126044 & 7.41695 \\
\hline$y-143$ & $y^{-}$ & 143 & 17104.12 & 17104.15 & 0.027268 & 1.594238 \\
\hline x144 & $x$ & 144 & 17245.15 & 17245.15 & 0.004672 & 0.270917 \\
\hline$y-148$ & $y^{-}$ & 148 & 17700.45 & 17700.47 & 0.021227 & 1.199237 \\
\hline$x+148$ & $x+$ & 148 & 17728.44 & 17728.51 & 0.065619 & 3.701338 \\
\hline z150 & $z$ & 150 & 17899.57 & 17899.54 & -0.03365 & -1.88021 \\
\hline
\end{tabular}




$\begin{array}{llrrrrr}\mathrm{y} 151 & \mathrm{y} & 151 & 18028.67 & 18028.68 & 0.008697 & 0.482398 \\ \mathrm{z} 154 & \mathrm{z} & 154 & 18298.82 & 18298.85 & 0.036056 & 1.970401 \\ \mathrm{x}+156 & \mathrm{x}+ & 156 & 18497.91 & 18497.99 & 0.074609 & 4.033372 \\ \mathrm{x} 157 & \mathrm{x} & 157 & 18553.93 & 18553.91 & -0.01593 & -0.85863 \\ \mathrm{z} 160 & \mathrm{z} & 160 & 18743 & 18743 & -0.00021 & -0.01126 \\ \mathrm{x} 160 & \mathrm{x} & 160 & 18784.99 & 18785.06 & 0.0667 & 3.550706 \\ \mathrm{x}+163 & \mathrm{x}+ & 163 & 19083.21 & 19083.25 & 0.044075 & 2.309621 \\ \mathrm{z} 164 & \mathrm{z} & 164 & 19153.29 & 19153.31 & 0.022266 & 1.162516 \\ \mathrm{x} 164 & \mathrm{x} & 164 & 19195.28 & 19195.38 & 0.10114 & 5.269003 \\ \mathrm{z} 165 & \mathrm{z} & 165 & 19281.38 & 19281.54 & 0.160996 & 8.349817\end{array}$


Table S2D. Summary of UVPD mass spectrum for G12C•GDPnP complex: fragment ions incorporating mass shift of +542.9526 Da (GDPnp)

\begin{tabular}{|c|c|c|c|c|c|c|}
\hline Ion & $\frac{\text { Ion }}{\text { Type }}$ & Residue & $\begin{array}{l}\text { Theoretical } \\
\text { Mass }\end{array}$ & $\begin{array}{l}\text { Experimental } \\
\text { Mass }\end{array}$ & $\Delta$ mass & $\begin{array}{l}\text { ppm } \\
\text { error }\end{array}$ \\
\hline c19 & c & 19 & 2409.922 & 2409.943 & 0.021029 & 8.726009 \\
\hline$a+125$ & $a+$ & 125 & 14644.87 & 14644.98 & 0.104671 & 7.147278 \\
\hline$a+140$ & $a+$ & 140 & 16290.71 & 16290.78 & 0.062315 & 3.825183 \\
\hline b143 & $b$ & 143 & 16674.91 & 16674.95 & 0.043084 & 2.583763 \\
\hline b158 & $b$ & 158 & 18343.68 & 18343.84 & 0.154344 & 8.414015 \\
\hline$a+158$ & $a+$ & 158 & 18316.69 & 18316.87 & 0.17537 & 9.574324 \\
\hline a160 & a & 160 & 18529.82 & 18529.96 & 0.141298 & 7.625439 \\
\hline c160 & c & 160 & 18574.84 & 18575 & 0.158658 & 8.541555 \\
\hline a162 & a & 162 & 18784.99 & 18785.06 & 0.073585 & 3.917224 \\
\hline$a+165$ & $a+$ & 165 & 19184.22 & 19184.38 & 0.154162 & 8.035872 \\
\hline z71 & $z$ & 71 & 8643.208 & 8643.184 & -0.02353 & -2.72214 \\
\hline z74 & z & 74 & 9091.415 & 9091.447 & 0.032381 & 3.561712 \\
\hline y80 & y & 80 & 9885.774 & 9885.834 & 0.060606 & 6.130628 \\
\hline$x+87$ & $x+$ & 87 & 10641.14 & 10641.18 & 0.03719 & 3.494923 \\
\hline$x 117$ & $x$ & 117 & 14079.69 & 14079.72 & 0.032666 & 2.32008 \\
\hline y119 & y & 119 & 14269.8 & 14269.85 & 0.054179 & 3.796759 \\
\hline y121 & y & 121 & 14499.89 & 14499.94 & 0.049358 & 3.404026 \\
\hline z123 & $z$ & 123 & 14655.92 & 14655.99 & 0.064645 & 4.410845 \\
\hline$y-123$ & $y^{-}$ & 123 & 14670.93 & 14671 & 0.064692 & 4.409538 \\
\hline y124 & $y$ & 124 & 14785.02 & 14785.04 & 0.012113 & 0.819275 \\
\hline x124 & $x$ & 124 & 14811 & 14811.07 & 0.066784 & 4.50908 \\
\hline$x+125$ & $x+$ & 125 & 14911.08 & 14911.18 & 0.101633 & 6.815937 \\
\hline$x+126$ & $x+$ & 126 & 15010.15 & 15010.21 & 0.060322 & 4.018746 \\
\hline$y-127$ & $y^{-}$ & 127 & 15110.21 & 15110.26 & 0.044265 & 2.929478 \\
\hline z127 & $z$ & 127 & 15095.2 & 15095.28 & 0.08206 & 5.436165 \\
\hline y128 & $y$ & 128 & 15239.31 & 15239.34 & 0.030835 & 2.023385 \\
\hline$x+128$ & $x+$ & 128 & 15266.3 & 15266.34 & 0.037154 & 2.433724 \\
\hline y129 & $y$ & 129 & 15395.41 & 15395.45 & 0.037977 & 2.466773 \\
\hline$x 129$ & $x$ & 129 & 15421.39 & 15421.44 & 0.042397 & 2.749233 \\
\hline x130 & $x$ & 130 & 15584.46 & 15584.51 & 0.048671 & 3.123047 \\
\hline x131 & $x$ & 131 & 15671.49 & 15671.55 & 0.064534 & 4.117924 \\
\hline x132 & $x$ & 132 & 15786.52 & 15786.56 & 0.044283 & 2.805115 \\
\hline$x+133$ & $x+$ & 133 & 15916.57 & 15916.59 & 0.025708 & 1.61517 \\
\hline y136 & $y$ & 136 & 16200.76 & 16200.88 & 0.114807 & 7.086517 \\
\hline y137 & $y$ & 137 & 16315.79 & 16315.86 & 0.070777 & 4.337945 \\
\hline$y-138$ & $y-$ & 138 & 16477.85 & 16477.97 & 0.119276 & 7.238569 \\
\hline x139 & $x$ & 139 & 16633.88 & 16634 & 0.127682 & 7.676022 \\
\hline z139 & $z$ & 139 & 16591.88 & 16592.01 & 0.135966 & 8.194732 \\
\hline
\end{tabular}




$\begin{array}{llrrrrr}\text { y140 } & y & 140 & 16722.92 & 16722.99 & 0.063405 & 3.791502 \\ \text { x+141 } & x+ & 141 & 16848.98 & 16849.02 & 0.043061 & 2.555702 \\ \text { z141 } & z & 141 & 16805.97 & 16806.04 & 0.064541 & 3.840361 \\ \text { y-142 } & y- & 142 & 16968.05 & 16968.15 & 0.097984 & 5.774618 \\ \text { y144 } & y & 144 & 17220.16 & 17220.22 & 0.061561 & 3.574937 \\ \text { z148 } & z & 148 & 17686.43 & 17686.46 & 0.029713 & 1.679989 \\ \text { x148 } & x & 148 & 17728.43 & 17728.51 & 0.081244 & 4.582696 \\ \text { x+149 } & x+ & 149 & 17842.52 & 17842.58 & 0.064066 & 3.590635 \\ \text { y-151 } & y- & 151 & 18028.66 & 18028.68 & 0.024322 & 1.349076 \\ \text { x156 } & x & 156 & 18497.9 & 18497.99 & 0.090234 & 4.878068 \\ \text { y160 } & y & 160 & 18760.01 & 18760.11 & 0.104302 & 5.559806 \\ \text { x+161 } & x+ & 161 & 18886.06 & 18886.15 & 0.0864 & 4.5748 \\ \text { x163 } & x & 163 & 19083.19 & 19083.25 & 0.0597 & 3.128408 \\ \text { x+163 } & x+ & 163 & 19084.2 & 19084.37 & 0.171404 & 8.98146 \\ z 165 & z & 165 & 19282.37 & 19282.48 & 0.105272 & 5.459494\end{array}$


Table S3A. Summary of UVPD mass spectrum for G12S•GDP complex: apo fragment ions

Column "Ions" is the fragment ion identity $(a, b, c, x, y, z)$ along with the number of residues in the fragment ion. A plus or minus sign indicates the fragment ion has one additional hydrogen atom or one less hydrogen atom than a conventional fragment ion. Column "Ion Type" is the type of fragment ion $(a, b, c, x, y, z)$. A plus sign indicates a fragment ion $(a, x)$ with an additional hydrogen atom; a minus sign indicates a fragment ion ( $y$ ) that is missing one hydrogen atom (the addition of one hydrogen atom for $a / x$ ions is not uncommon in UVPD mass spectra of intact proteins); Column "Residue" is the number of residues in the fragment ion. Column "Theoretical Mass" is the theoretical mass of the fragment ion; Column "Experimental Mass" is the experimentally measured mass of the fragment ion. Column "Delta mass" is the mass difference between the theoretical and experimental masses; and column "ppm error" is the mass error in ppm. The "apo" table (Table S3A) provides the fragment ions that do not retain the ligand. Tables S3B, S3C, and S3D provide the fragment ions that incorporate the ligand based on the mass shift corresponding to the retained ligand (with or without an additional hydrogen atom).

\begin{tabular}{|c|c|c|c|c|c|c|}
\hline & $\underline{\text { Ion }}$ & & Theoretical & Experimental & & $\mathrm{ppm}$ \\
\hline Ion & Type & $\underline{\text { Residue }}$ & Mass & $\underline{\text { Mass }}$ & $\Delta$ mass & error \\
\hline b4 & b & 4 & 418.1522 & 418.153 & 0.000812 & 1.941877 \\
\hline c4 & c & 4 & 435.1785 & 435.1795 & 0.000971 & 2.231268 \\
\hline a5 & a & 5 & 553.2206 & 553.2216 & 0.00097 & 1.753369 \\
\hline b5 & $b$ & 5 & 581.2156 & 581.2166 & 0.00105 & 1.806559 \\
\hline$a+6$ & $a+$ & 6 & 682.3234 & 682.3246 & 0.001152 & 1.688302 \\
\hline b7 & $b$ & 7 & 822.3946 & 822.3956 & 0.000985 & 1.197722 \\
\hline$a+7$ & $a+$ & 7 & 795.4075 & 795.4091 & 0.001625 & 2.042938 \\
\hline a8 & a & 8 & 893.4681 & 893.4693 & 0.001221 & 1.366585 \\
\hline b8 & $b$ & 8 & 921.463 & 921.4643 & 0.001362 & 1.478084 \\
\hline$a+8$ & $a+$ & 8 & 894.4759 & 894.4775 & 0.001636 & 1.828968 \\
\hline b9 & $\mathrm{b}$ & 9 & 1020.531 & 1020.533 & 0.001189 & 1.165079 \\
\hline$a+9$ & $a+$ & 9 & 993.5443 & 993.5456 & 0.001341 & 1.349681 \\
\hline c9 & c & 9 & 1037.558 & 1037.56 & 0.002172 & 2.093378 \\
\hline b10 & $b$ & 10 & 1119.6 & 1119.601 & 0.0012 & 1.071812 \\
\hline a10 & a & 10 & 1091.605 & 1091.606 & 0.001364 & 1.249536 \\
\hline c10 & c & 10 & 1136.626 & 1136.628 & 0.001999 & 1.758714 \\
\hline$a+11$ & $a+$ & 11 & 1149.634 & 1149.635 & 0.001254 & 1.090754 \\
\hline b11 & $b$ & 11 & 1176.621 & 1176.623 & 0.001834 & 1.5587 \\
\hline c11 & c & 11 & 1193.648 & 1193.65 & 0.002024 & 1.695643 \\
\hline b12 & $b$ & 12 & 1247.658 & 1247.659 & 0.000613 & 0.49132 \\
\hline$a+12$ & $a+$ & 12 & 1220.671 & 1220.672 & 0.000887 & 0.726623 \\
\hline c12 & c & 12 & 1264.685 & 1264.686 & 0.001291 & 1.020808 \\
\hline$a+13$ & $a+$ & 13 & 1307.703 & 1307.704 & 0.000961 & 0.734852 \\
\hline c13 & $c$ & 13 & 1351.717 & 1351.718 & 0.000999 & 0.73906 \\
\hline a13 & a & 13 & 1306.695 & 1306.697 & 0.001706 & 1.305583 \\
\hline c14 & c & 14 & 1408.738 & 1408.739 & 0.000657 & 0.466375 \\
\hline
\end{tabular}




\begin{tabular}{|c|c|c|c|c|c|c|}
\hline$a+14$ & $a+$ & 14 & 1364.725 & 1364.726 & 0.001108 & 0.811862 \\
\hline b14 & b & 14 & 1391.712 & 1391.714 & 0.001933 & 1.388937 \\
\hline$a+15$ & $a+$ & 15 & 1463.793 & 1463.793 & $\begin{array}{r}-4.20 \mathrm{E}- \\
05\end{array}$ & -0.02871 \\
\hline a15 & a & 15 & 1462.785 & 1462.787 & 0.001558 & 1.065091 \\
\hline$a+16$ & $a+$ & 16 & 1520.815 & 1520.815 & 0.000715 & 0.470122 \\
\hline c16 & c & 16 & 1564.828 & 1564.83 & 0.001607 & 1.02695 \\
\hline b16 & $b$ & 16 & 1547.802 & 1547.805 & 0.003249 & 2.099106 \\
\hline c17 & c & 17 & 1692.923 & 1692.921 & -0.0018 & -1.06325 \\
\hline$a+17$ & $a+$ & 17 & 1648.91 & 1648.91 & 0.000285 & 0.172822 \\
\hline a17 & $a$ & 17 & 1647.902 & 1647.905 & 0.002983 & 1.810181 \\
\hline$a+18$ & $a+$ & 18 & 1735.942 & 1735.942 & $\begin{array}{r}-5.40 \mathrm{E}- \\
05\end{array}$ & -0.03113 \\
\hline$a+19$ & $a+$ & 19 & 1806.979 & 1806.979 & 0.000311 & 0.172093 \\
\hline a19 & $a$ & 19 & 1805.971 & 1805.973 & 0.001592 & 0.88152 \\
\hline$a+20$ & $a+$ & 20 & 1920.063 & 1920.062 & -0.00033 & -0.17032 \\
\hline c20 & C & 20 & 1964.076 & 1964.078 & 0.001983 & 1.009635 \\
\hline a21 & $a$ & 21 & 2020.103 & 2020.104 & 0.000908 & 0.449482 \\
\hline b21 & $b$ & 21 & 2048.098 & 2048.098 & 0.000914 & 0.446268 \\
\hline$a+22$ & $a+$ & 22 & 2134.195 & 2134.194 & -0.00065 & -0.30224 \\
\hline c22 & c & 22 & 2178.208 & 2178.21 & 0.002126 & 0.976032 \\
\hline a22 & a & 22 & 2133.187 & 2133.189 & 0.002589 & 1.213677 \\
\hline$a+23$ & $a+$ & 23 & 2262.253 & 2262.252 & -0.00107 & -0.47388 \\
\hline a23 & $a$ & 23 & 2261.245 & 2261.247 & 0.002115 & 0.935325 \\
\hline c23 & c & 23 & 2306.267 & 2306.269 & 0.002384 & 1.033705 \\
\hline b23 & $b$ & 23 & 2289.24 & 2289.243 & 0.003049 & 1.331883 \\
\hline$a+24$ & $a+$ & 24 & 2375.337 & 2375.337 & -0.00042 & -0.17473 \\
\hline b24 & $b$ & 24 & 2402.324 & 2402.326 & 0.001997 & 0.831278 \\
\hline c24 & c & 24 & 2419.351 & 2419.355 & 0.004261 & 1.761216 \\
\hline c25 & c & 25 & 2532.435 & 2532.435 & 0.000768 & 0.303265 \\
\hline b25 & b & 25 & 2515.408 & 2515.41 & 0.001433 & 0.569689 \\
\hline$a+27$ & $a+$ & 27 & 2730.523 & 2730.522 & -0.00044 & -0.16042 \\
\hline a27 & $\mathrm{a}$ & 27 & 2729.515 & 2729.517 & 0.002016 & 0.738593 \\
\hline c27 & c & 27 & 2774.536 & 2774.539 & 0.003018 & 1.087749 \\
\hline c28 & c & 28 & 2911.595 & 2911.585 & -0.01004 & -3.44863 \\
\hline$a+28$ & $a+$ & 28 & 2867.582 & 2867.582 & 0.000711 & 0.247933 \\
\hline b28 & $b$ & 28 & 2894.569 & 2894.577 & 0.007761 & 2.681228 \\
\hline a29 & $a$ & 29 & 3013.642 & 3013.647 & 0.005019 & 1.665427 \\
\hline c29 & c & 29 & 3058.663 & 3058.672 & 0.008266 & 2.702488 \\
\hline$a+30$ & $a+$ & 30 & 3113.718 & 3113.718 & -0.00061 & -0.19624 \\
\hline c30 & c & 30 & 3157.732 & 3157.732 & 0.000159 & 0.050353 \\
\hline a31 & $a$ & 31 & 3227.738 & 3227.74 & 0.002002 & 0.620249 \\
\hline c31 & c & 31 & 3272.759 & 3272.764 & 0.004957 & 1.514624 \\
\hline
\end{tabular}




\begin{tabular}{|c|c|c|c|c|c|c|}
\hline c32 & c & 32 & 3401.801 & 3401.807 & 0.005824 & 1.712034 \\
\hline c33 & c & 33 & 3564.865 & 3564.86 & -0.00502 & -1.40707 \\
\hline$a+33$ & $a+$ & 33 & 3520.851 & 3520.851 & -0.00066 & -0.18718 \\
\hline$a+34$ & $a+$ & 34 & 3635.878 & 3635.88 & 0.001942 & 0.534112 \\
\hline$a+35$ & $a+$ & 35 & 3732.931 & 3732.931 & 0.000452 & 0.121076 \\
\hline c35 & c & 35 & 3776.944 & 3776.949 & 0.004884 & 1.293109 \\
\hline a37 & $a$ & 37 & 3946.055 & 3946.061 & 0.006175 & 1.564854 \\
\hline c38 & c & 38 & 4120.119 & 4120.118 & -0.0005 & -0.1216 \\
\hline a38 & a & 38 & 4075.098 & 4075.098 & 0.000939 & 0.230424 \\
\hline a39 & a & 39 & 4190.124 & 4190.118 & -0.00623 & -1.48587 \\
\hline b39 & $b$ & 39 & 4218.119 & 4218.125 & 0.005207 & 1.234436 \\
\hline a40 & $a$ & 40 & 4277.157 & 4277.16 & 0.003248 & 0.759383 \\
\hline c40 & c & 40 & 4322.178 & 4322.181 & 0.003592 & 0.831063 \\
\hline a41 & a & 41 & 4440.22 & 4440.22 & $\begin{array}{r}-2.30 \mathrm{E}- \\
05\end{array}$ & -0.00518 \\
\hline$a+42$ & $a+$ & 42 & 4597.329 & 4597.332 & 0.003559 & 0.774138 \\
\hline a42 & $a$ & 42 & 4596.321 & 4596.325 & 0.004017 & 0.87396 \\
\hline$c 42$ & c & 42 & 4641.342 & 4641.35 & 0.007704 & 1.659865 \\
\hline$a+43$ & $a+$ & 43 & 4725.424 & 4725.424 & 0.000109 & 0.02306 \\
\hline a44 & $a$ & 44 & 4852.474 & 4852.475 & 1.30E-05 & 0.002679 \\
\hline a45 & a & 45 & 4951.543 & 4951.548 & 0.005578 & 1.126518 \\
\hline a46 & a & 46 & 5050.611 & 5050.614 & 0.003086 & 0.611015 \\
\hline a47 & a & 47 & 5163.695 & 5163.7 & 0.004231 & 0.819374 \\
\hline a49 & a & 49 & 5335.744 & 5335.745 & 0.000997 & 0.186853 \\
\hline$a+50$ & $a+$ & 50 & 5465.794 & 5465.793 & -0.00169 & -0.30902 \\
\hline$a+51$ & $a+$ & 51 & 5566.842 & 5566.841 & -0.00042 & -0.07527 \\
\hline a53 & $a$ & 53 & 5781.927 & 5781.928 & 0.000826 & 0.142859 \\
\hline a54 & a & 54 & 5895.011 & 5895.013 & 0.001239 & 0.210178 \\
\hline a55 & $a$ & 55 & 6010.038 & 6010.045 & 0.006888 & 1.146083 \\
\hline$a+56$ & $a+$ & 56 & 6124.13 & 6124.114 & -0.01602 & -2.61654 \\
\hline a57 & $a$ & 57 & 6236.206 & 6236.2 & -0.00608 & -0.97447 \\
\hline$a+58$ & $a+$ & 58 & 6352.241 & 6352.233 & -0.00837 & -1.31781 \\
\hline c58 & c & 58 & 6396.255 & 6396.249 & -0.0054 & -0.84487 \\
\hline$a+60$ & $a+$ & 60 & 6524.326 & 6524.325 & -0.00112 & -0.17167 \\
\hline$a+61$ & $a+$ & 61 & 6581.347 & 6581.347 & -0.00014 & -0.02082 \\
\hline a62 & $a$ & 62 & 6708.398 & 6708.403 & 0.004616 & 0.688093 \\
\hline c63 & c & 63 & 6882.462 & 6882.406 & -0.05555 & -8.07124 \\
\hline$c 64$ & c & 64 & 7011.505 & 7011.454 & -0.05087 & -7.25479 \\
\hline a64 & a & 64 & 6966.483 & 6966.485 & 0.001955 & 0.280629 \\
\hline a69 & a & 69 & 7574.757 & 7574.769 & 0.011798 & 1.557542 \\
\hline a70 & $a$ & 70 & 7689.784 & 7689.78 & -0.00391 & -0.5086 \\
\hline$a+71$ & $a+$ & 71 & 7818.851 & 7818.852 & 0.001461 & 0.186852 \\
\hline c72 & c & 72 & 8025.928 & 8025.89 & -0.03717 & -4.63161 \\
\hline
\end{tabular}




\begin{tabular}{|c|c|c|c|c|c|c|}
\hline a72 & $a$ & 72 & 7980.906 & 7980.9 & -0.00619 & -0.77585 \\
\hline a73 & a & 73 & 8111.947 & 8111.936 & -0.01104 & -1.36071 \\
\hline c74 & c & 74 & 8313.069 & 8313.054 & -0.01466 & -1.76385 \\
\hline$a+74$ & $a+$ & 74 & 8269.056 & 8269.071 & 0.015573 & 1.883283 \\
\hline a75 & $\mathrm{a}$ & 75 & 8369.096 & 8369.109 & 0.013433 & 1.605072 \\
\hline a77 & a & 77 & 8555.16 & 8555.17 & 0.010632 & 1.242759 \\
\hline b78 & $b$ & 78 & 8640.176 & 8640.129 & -0.04694 & -5.43288 \\
\hline a78 & a & 78 & 8612.181 & 8612.192 & 0.01119 & 1.299322 \\
\hline a79 & a & 79 & 8759.249 & 8759.264 & 0.014059 & 1.605046 \\
\hline$a 80$ & a & 80 & 8872.334 & 8872.349 & 0.015326 & 1.727392 \\
\hline c82 & c & 82 & 9119.432 & 9119.421 & -0.01135 & -1.24416 \\
\hline a82 & a & 82 & 9074.411 & 9074.425 & 0.013531 & 1.491116 \\
\hline a83 & a & 83 & 9221.48 & 9221.489 & 0.009522 & 1.032589 \\
\hline c87 & c & 87 & 9678.708 & 9678.696 & -0.01162 & -1.20099 \\
\hline a87 & $a$ & 87 & 9633.687 & 9633.696 & 0.009523 & 0.98851 \\
\hline$a+88$ & $a+$ & 88 & 9735.742 & 9735.745 & 0.002494 & 0.256166 \\
\hline$a+90$ & $a+$ & 90 & 9950.869 & 9950.84 & -0.029 & -2.91412 \\
\hline a91 & $a$ & 91 & 10096.93 & 10096.94 & 0.005641 & 0.558685 \\
\hline a92 & a & 92 & 10225.97 & 10225.98 & 0.003923 & 0.383631 \\
\hline a104 & $a$ & 104 & 11800.82 & 11800.83 & 0.017973 & 1.52303 \\
\hline$a+125$ & $a+$ & 125 & 14085.94 & 14085.89 & -0.05082 & -3.60778 \\
\hline c133 & c & 133 & 15015.37 & 15015.24 & -0.13038 & -8.68317 \\
\hline b134 & $b$ & 134 & 15111.43 & 15111.3 & -0.12924 & -8.5528 \\
\hline c168 & c & 168 & 19062.56 & 19062.37 & -0.18611 & -9.76312 \\
\hline y3 & y & 3 & 403.2431 & 403.2439 & 0.000808 & 2.003754 \\
\hline y4 & $y$ & 4 & 540.302 & 540.3029 & 0.000919 & 1.700901 \\
\hline$x 4$ & $x$ & 4 & 566.2813 & 566.2822 & 0.000963 & 1.700568 \\
\hline$x 6$ & $x$ & 6 & 850.4773 & 850.4786 & 0.001304 & 1.533257 \\
\hline y6 & $y$ & 6 & 824.4981 & 824.4996 & 0.001565 & 1.898125 \\
\hline z6 & $z$ & 6 & 808.4793 & 808.4811 & 0.001837 & 2.272167 \\
\hline y7 & y & 7 & 937.5821 & 937.5832 & 0.001124 & 1.198828 \\
\hline$x 7$ & $x$ & 7 & 963.5614 & 963.5639 & 0.00251 & 2.60492 \\
\hline z8 & $z$ & 8 & 1050.606 & 1050.608 & 0.002262 & 2.153043 \\
\hline y8 & y & 8 & 1066.625 & 1066.627 & 0.002296 & 2.152585 \\
\hline$x+8$ & $x+$ & 8 & 1093.612 & 1093.618 & 0.006295 & 5.756127 \\
\hline y9 & $y$ & 9 & 1222.726 & 1222.727 & 0.001283 & 1.049295 \\
\hline$x 9$ & $x$ & 9 & 1248.705 & 1248.707 & 0.001877 & 1.503157 \\
\hline$x 10$ & $x$ & 10 & 1347.773 & 1347.774 & 0.000972 & 0.721189 \\
\hline y10 & $y$ & 10 & 1321.794 & 1321.796 & 0.001843 & 1.394317 \\
\hline$x 12$ & $x$ & 12 & 1561.905 & 1561.907 & 0.001434 & 0.918109 \\
\hline$x+13$ & $x+$ & 13 & 1725.976 & 1725.974 & -0.00217 & -1.2567 \\
\hline$x 13$ & $x$ & 13 & 1724.969 & 1724.972 & 0.003703 & 2.146706 \\
\hline$x+14$ & $x+$ & 14 & 1873.045 & 1873.044 & -0.00112 & -0.59851 \\
\hline
\end{tabular}




$\begin{array}{llrrrrr}\text { x14 } & x & 14 & 1872.037 & 1872.039 & 0.002066 & 1.103611 \\ y-15 & y- & 15 & 1916.087 & 1916.085 & -0.0023 & -1.20035 \\ \text { y15 } & y & 15 & 1917.095 & 1917.094 & -0.00077 & -0.40321 \\ \text { x+15 } & x+ & 15 & 1944.082 & 1944.082 & -2.30 E- & -0.01185 \\ \text { y16 } & y & 16 & 2032.122 & 2032.124 & 0.002364 & 1.163316 \\ \text { y19 } & y & 19 & 2303.239 & 2303.241 & 0.002253 & 0.978188 \\ \text { x+22 } & x+ & 22 & 2715.433 & 2715.43 & -0.003 & -1.10407 \\ x+28 & x+ & 28 & 3344.771 & 3344.771 & -0.00085 & -0.25474 \\ x+29 & x+ & 29 & 3491.84 & 3491.834 & -0.00627 & -1.79677 \\ z 29 & z & 29 & 3448.834 & 3448.838 & 0.003732 & 1.082105 \\ x 29 & x & 29 & 3490.832 & 3490.838 & 0.005749 & 1.646885 \\ y-37 & y- & 37 & 4321.321 & 4321.329 & 0.007755 & 1.794598 \\ x+41 & x+ & 41 & 4791.497 & 4791.504 & 0.006884 & 1.436705 \\ y 43 & y & 43 & 4993.653 & 4993.656 & 0.003766 & 0.754157 \\ x+45 & x+ & 45 & 5234.735 & 5234.738 & 0.003317 & 0.633646 \\ x+46 & x+ & 46 & 5335.783 & 5335.745 & -0.03789 & -7.10168 \\ x+55 & x+ & 55 & 6306.248 & 6306.242 & -0.00576 & -0.91354 \\ z 169 & z & 169 & 19247.64 & 19247.45 & -0.18211 & -9.46127 \\ x 169 & x & 169 & 19289.63 & 19289.47 & -0.1691 & -8.76642 \\ y 169 & y & 169 & 19263.66 & 19263.49 & -0.16508 & -8.56951\end{array}$


Table S3B. Summary of UVPD mass spectrum for G12S•GDP complex: fragment ions incorporating mass shift of +464.9937 Da (GDP)

\begin{tabular}{|c|c|c|c|c|c|c|}
\hline & $\underline{\text { Ion }}$ & & Theoretical & Experimental & & $\mathrm{ppm}$ \\
\hline Ion & Type & Residue & $\underline{\text { Mass }}$ & Mass & $\Delta$ mass & error \\
\hline b27 & $b$ & 27 & 3222.504 & 3222.529 & 0.025072 & 7.780286 \\
\hline$c 27$ & C & 27 & 3239.53 & 3239.559 & 0.028801 & 8.890488 \\
\hline$a+56$ & $a+$ & 56 & 6589.124 & 6589.181 & 0.056879 & 8.632251 \\
\hline a125 & $a$ & 125 & 14549.93 & 14549.93 & -0.00026 & -0.01766 \\
\hline c135 & c & 135 & 15664.49 & 15664.5 & 0.012497 & 0.797792 \\
\hline$a+142$ & $a+$ & 142 & 16440.9 & 16440.85 & -0.05017 & -3.05154 \\
\hline c143 & $c$ & 143 & 16598 & 16597.98 & -0.01463 & -0.88149 \\
\hline c146 & c & 146 & 16915.12 & 16915.03 & -0.08468 & -5.00647 \\
\hline c151 & c & 151 & 17499.46 & 17499.39 & -0.0705 & -4.02858 \\
\hline a154 & a & 154 & 17725.55 & 17725.52 & -0.03798 & -2.14284 \\
\hline a159 & a & 159 & 18322.8 & 18322.83 & 0.03593 & 1.960945 \\
\hline a160 & a & 160 & 18435.88 & 18435.91 & 0.024131 & 1.308915 \\
\hline$a+161$ & $a+$ & 161 & 18535.96 & 18535.99 & 0.03042 & 1.641133 \\
\hline a163 & $a$ & 163 & 18820.09 & 18820.11 & 0.017272 & 0.917743 \\
\hline a165 & a & 165 & 19089.28 & 19089.37 & 0.087577 & 4.587758 \\
\hline b165 & $b$ & 165 & 19117.27 & 19117.39 & 0.112644 & 5.892263 \\
\hline b167 & $b$ & 167 & 19382.43 & 19382.57 & 0.146819 & 7.574851 \\
\hline c168 & c & 168 & 19527.55 & 19527.61 & 0.056865 & 2.91204 \\
\hline$y-43$ & $y-$ & 43 & 5457.638 & 5457.641 & 0.002788 & 0.51085 \\
\hline $\mathrm{z} 46$ & $z$ & 46 & 5757.771 & 5757.751 & -0.01993 & -3.46089 \\
\hline y47 & y & 47 & 5929.89 & 5929.857 & -0.03336 & -5.62506 \\
\hline y48 & $y$ & 48 & 6016.922 & 6016.894 & -0.02872 & -4.77254 \\
\hline x52 & $x$ & 52 & 6471.075 & 6471.097 & 0.022633 & 3.497564 \\
\hline$x 53$ & $x$ & 53 & 6599.17 & 6599.191 & 0.020935 & 3.172369 \\
\hline$x+79$ & $x+$ & 79 & 9687.733 & 9687.705 & -0.02821 & -2.91193 \\
\hline$x 86$ & $x$ & 86 & 10491.14 & 10491.12 & -0.01813 & -1.72841 \\
\hline$x+87$ & $x+$ & 87 & 10563.18 & 10563.16 & -0.0243 & -2.30045 \\
\hline x99 & $x$ & 99 & 11965.81 & 11965.75 & -0.05815 & -4.85993 \\
\hline z100 & $z$ & 100 & 12051.87 & 12051.84 & -0.03179 & -2.63768 \\
\hline x100 & $x$ & 100 & 12093.87 & 12093.87 & -0.00345 & -0.28543 \\
\hline x101 & $x$ & 101 & 12208.9 & 12208.88 & -0.01542 & -1.26326 \\
\hline$x 118$ & $x$ & 118 & 14114.81 & 14114.8 & -0.01559 & -1.10444 \\
\hline$x+119$ & $x+$ & 119 & 14218.83 & 14218.8 & -0.02748 & -1.93251 \\
\hline$x+120$ & $x+$ & 120 & 14319.88 & 14319.86 & -0.01681 & -1.17376 \\
\hline$x 123$ & $x$ & 123 & 14619.96 & 14619.99 & 0.031863 & 2.179418 \\
\hline$y-124$ & $y-$ & 124 & 14706.06 & 14705.93 & -0.12639 & -8.59414 \\
\hline$x+124$ & $x+$ & 124 & 14734.05 & 14734.05 & -0.00363 & -0.24603 \\
\hline
\end{tabular}




$\begin{array}{llrrrrr}\text { y-125 } & y- & 125 & 14805.13 & 14805.1 & -0.02487 & -1.68009 \\ \text { x125 } & x & 125 & 14832.11 & 14832.11 & -0.00391 & -0.26348 \\ \text { x126 } & x & 126 & 14931.18 & 14931.08 & -0.10381 & -6.9527 \\ \text { y126 } & y & 126 & 14905.2 & 14905.18 & -0.01688 & -1.13256 \\ \text { x127 } & x & 127 & 15059.24 & 15059.25 & 0.008995 & 0.597308 \\ \text { y-128 } & y- & 128 & 15160.35 & 15160.33 & -0.01733 & -1.14324 \\ \text { x128 } & x & 128 & 15187.33 & 15187.32 & -0.01101 & -0.72521 \\ \text { x129 } & x & 129 & 15343.44 & 15343.4 & -0.03468 & -2.26051 \\ \text { y-132 } & y- & 132 & 15681.57 & 15681.54 & -0.03404 & -2.17064 \\ \text { x+132 } & x+ & 132 & 15709.57 & 15709.58 & 0.013902 & 0.884937 \\ \text { x133 } & x & 133 & 15837.6 & 15837.58 & -0.01551 & -0.97906 \\ \text { x135 } & x & 135 & 16051.73 & 16051.78 & 0.045151 & 2.812843 \\ \text { x139 } & x & 139 & 16555.92 & 16555.91 & -0.0101 & -0.60993 \\ \text { y140 } & y & 140 & 16644.97 & 16644.92 & -0.04361 & -2.62019 \\ \text { x141 } & x & 141 & 16770.01 & 16770 & -0.00974 & -0.5811 \\ \text { y-142 } & y- & 142 & 16890.09 & 16890.12 & 0.026123 & 1.546648 \\ \text { z143 } & z & 143 & 17012.14 & 17012.15 & 0.011356 & 0.667523 \\ \text { y144 } & y & 144 & 17142.2 & 17142.18 & -0.02641 & -1.54088 \\ \text { y-150 } & y- & 150 & 17837.61 & 17837.63 & 0.015855 & 0.888854 \\ y-152 & y- & 152 & 18021.73 & 18021.7 & -0.03403 & -1.88805 \\ \text { z153 } & z & 153 & 18093.76 & 18093.74 & -0.01117 & -0.61734 \\ \text { y155 } & y & 155 & 18294.89 & 18294.81 & -0.08263 & -4.51683 \\ \text { x+156 } & x+ & 156 & 18420.95 & 18420.9 & -0.04585 & -2.48902 \\ \text { x157 } & x & 157 & 18476.96 & 18476.93 & -0.03385 & -1.83201 \\ \text { y-157 } & y- & 157 & 18449.97 & 18449.94 & -0.0282 & -1.52873 \\ \text { y-161 } & y- & 161 & 18764.13 & 18764.11 & -0.02291 & -1.22084 \\ \text { x162 } & x & 162 & 18890.19 & 18890.17 & -0.01518 & -0.80338 \\ \text { y164 } & y & 164 & 19076.36 & 19076.37 & 0.011546 & 0.605252 \\ \text { y165 } & y & 165 & 19204.46 & 19204.45 & -0.00571 & -0.29712 \\ \text { z165 } & z & 165 & 19188.44 & 19188.46 & 0.021557 & 1.123437 \\ \text { z167 } & z & 167 & 19480.54 & 19480.6 & 0.055577 & 2.852949 \\ \text { x+169 } & x+ & 19755.64 & 19755.69 & 0.055302 & 2.799301\end{array}$


Table S3C. Summary of UVPD mass spectrum for G12S•GDP complex: fragment ions incorporating mass shift of +462.9781 Da (GDP)

\begin{tabular}{|c|c|c|c|c|c|c|}
\hline & $\underline{\text { Ion }}$ & & Theoretical & Experimental & & $\mathrm{ppm}$ \\
\hline Ion & Type & Residue & Mass & Mass & $\Delta$ mass & error \\
\hline b1 & $b$ & 1 & 519.9996 & 520 & 0.00044 & 0.846155 \\
\hline$a+27$ & $a+$ & 27 & 3193.501 & 3193.529 & 0.028006 & 8.769676 \\
\hline 121 & $b$ & 121 & 14134.67 & 14134.78 & 0.104636 & 7.402788 \\
\hline b125 & $b$ & 125 & 14575.91 & 14575.88 & -0.02333 & -1.60079 \\
\hline b138 & $b$ & 138 & 16051.64 & 16051.78 & 0.132941 & 8.28208 \\
\hline b142 & b & 142 & 16465.87 & 16465.94 & 0.071778 & 4.359199 \\
\hline$a+144$ & $a+$ & 144 & 16681.01 & 16680.95 & -0.06478 & -3.8837 \\
\hline b148 & $b$ & 148 & 17095.21 & 17095.16 & -0.04448 & -2.60213 \\
\hline b161 & $b$ & 161 & 18560.93 & 18560.97 & 0.040864 & 2.201614 \\
\hline b162 & $b$ & 162 & 18717.03 & 18717.06 & 0.026424 & 1.411762 \\
\hline$a+164$ & $a+$ & 164 & 18932.17 & 18932.19 & 0.022362 & 1.181162 \\
\hline$a+166$ & $a+$ & 166 & 19216.37 & 19216.35 & -0.01601 & -0.8333 \\
\hline a166 & a & 166 & 19215.36 & 19215.44 & 0.084539 & 4.399554 \\
\hline a167 & a & 167 & 19352.42 & 19352.42 & 0.004867 & 0.251493 \\
\hline$a+167$ & $a+$ & 167 & 19353.43 & 19353.55 & 0.120212 & 6.211405 \\
\hline a168 & a & 168 & 19480.51 & 19480.6 & 0.085812 & 4.405018 \\
\hline b168 & $b$ & 168 & 19508.51 & 19508.66 & 0.153397 & 7.863082 \\
\hline a169 & a & 169 & 19609.55 & 19609.67 & 0.119394 & 6.088562 \\
\hline c169 & c & 169 & 19654.58 & 19654.7 & 0.119663 & 6.088302 \\
\hline y64 & $y$ & 64 & 7711.723 & 7711.769 & 0.045603 & 5.913464 \\
\hline$x 66$ & $x$ & 66 & 7980.824 & 7980.9 & 0.075713 & 9.486865 \\
\hline x98 & $x$ & 98 & 11800.73 & 11800.83 & 0.099878 & 8.463711 \\
\hline$y-100$ & $y^{-}$ & 100 & 12064.87 & 12064.87 & -0.00044 & -0.03663 \\
\hline$x+117$ & $x+$ & 117 & 14000.72 & 14000.71 & -0.00723 & -0.51626 \\
\hline$y-118$ & $y-$ & 118 & 14085.81 & 14085.89 & 0.082071 & 5.826505 \\
\hline y119 & $y$ & 119 & 14189.83 & 14189.8 & -0.02991 & -2.10813 \\
\hline z120 & $z$ & 120 & 14274.86 & 14274.82 & -0.03319 & -2.32542 \\
\hline y121 & $y$ & 121 & 14419.92 & 14419.89 & -0.02741 & -1.90091 \\
\hline$x 121$ & $x$ & 121 & 14445.9 & 14445.92 & 0.021156 & 1.464499 \\
\hline$y-122$ & $y-$ & 122 & 14475.93 & 14475.87 & -0.06204 & -4.28587 \\
\hline$x+122$ & $x+$ & 122 & 14503.93 & 14503.94 & 0.011891 & 0.819845 \\
\hline z123 & $z$ & 123 & 14575.95 & 14575.88 & -0.06217 & -4.26545 \\
\hline$y-123$ & $y-$ & 123 & 14590.96 & 14590.9 & -0.06091 & -4.17423 \\
\hline y127 & $y$ & 127 & 15031.24 & 15031.21 & -0.03935 & -2.61808 \\
\hline z127 & z & 127 & 15015.23 & 15015.24 & 0.018719 & 1.246668 \\
\hline x129 & $x$ & 129 & 15341.42 & 15341.4 & -0.02426 & -1.58121 \\
\hline y129 & y & 129 & 15315.44 & 15315.43 & -0.0144 & -0.94003 \\
\hline$x+130$ & $x+$ & 130 & 15505.49 & 15505.45 & -0.0439 & -2.83126 \\
\hline
\end{tabular}




\begin{tabular}{|c|c|c|c|c|c|c|}
\hline z132 & $z$ & 132 & 15664.54 & 15664.5 & -0.0419 & -2.67502 \\
\hline y133 & y & 133 & 15809.61 & 15809.58 & -0.02844 & -1.79884 \\
\hline$x+133$ & $x+$ & 133 & 15836.59 & 15836.57 & -0.02012 & -1.27041 \\
\hline y136 & y & 136 & 16120.79 & 16120.77 & -0.02153 & -1.33573 \\
\hline z136 & z & 136 & 16104.77 & 16104.78 & 0.011392 & 0.707368 \\
\hline z137 & z & 137 & 16219.8 & 16219.84 & 0.037675 & 2.322779 \\
\hline$x 137$ & $x$ & 137 & 16261.8 & 16261.84 & 0.048434 & 2.978392 \\
\hline$y-139$ & $y-$ & 139 & 16526.91 & 16526.91 & -0.00838 & -0.50735 \\
\hline z140 & $z$ & 140 & 16626.93 & 16626.91 & -0.01657 & -0.9967 \\
\hline$x+140$ & $x+$ & 140 & 16669.94 & 16669.94 & 0.005893 & 0.353509 \\
\hline z141 & $z$ & 141 & 16726 & 16725.98 & -0.0176 & -1.05219 \\
\hline y141 & y & 141 & 16742.02 & 16742.01 & -0.01097 & -0.65548 \\
\hline z142 & z & 142 & 16873.07 & 16873.03 & -0.04206 & -2.49297 \\
\hline$x 142$ & $x$ & 142 & 16915.07 & 16915.03 & -0.0313 & -1.85072 \\
\hline$x 143$ & $x$ & 143 & 17052.12 & 17052.14 & 0.014766 & 0.865933 \\
\hline$x+144$ & $x+$ & 144 & 17167.18 & 17167.13 & -0.04429 & -2.57993 \\
\hline$x+145$ & $x+$ & 145 & 17295.23 & 17295.22 & -0.01304 & -0.75385 \\
\hline y147 & $y$ & 147 & 17494.42 & 17494.38 & -0.03727 & -2.13045 \\
\hline$x+148$ & $x+$ & 148 & 17649.46 & 17649.45 & -0.0097 & -0.54948 \\
\hline z148 & $z$ & 148 & 17606.45 & 17606.49 & 0.033998 & 1.930996 \\
\hline$y-149$ & $y-$ & 149 & 17734.55 & 17734.53 & -0.01706 & -0.96174 \\
\hline$x+149$ & $x+$ & 149 & 17762.54 & 17762.53 & -0.01368 & -0.77016 \\
\hline$x+150$ & $x+$ & 150 & 17863.59 & 17863.66 & 0.064372 & 3.603529 \\
\hline$x+151$ & $x+$ & 151 & 17976.68 & 17976.66 & -0.01993 & -1.10877 \\
\hline x152 & $x$ & 152 & 18046.71 & 18046.64 & -0.06118 & -3.39009 \\
\hline$x+154$ & $x+$ & 154 & 18262.84 & 18262.86 & 0.014454 & 0.791441 \\
\hline y158 & $y$ & 158 & 18536 & 18535.99 & -0.00851 & -0.45938 \\
\hline y162 & $y$ & 162 & 18862.19 & 18862.16 & -0.03569 & -1.89225 \\
\hline$x+163$ & $x+$ & 163 & 18988.25 & 18988.24 & -0.00495 & -0.2609 \\
\hline z164 & z & 164 & 19058.33 & 19058.28 & -0.04653 & -2.44124 \\
\hline y165 & $y$ & 165 & 19202.44 & 19202.35 & -0.08914 & -4.64227 \\
\hline x165 & $x$ & 165 & 19228.42 & 19228.46 & 0.03976 & 2.067773 \\
\hline z165 & $z$ & 165 & 19186.42 & 19186.49 & 0.065769 & 3.427893 \\
\hline$y-166$ & $y-$ & 166 & 19364.5 & 19364.53 & 0.033021 & 1.705236 \\
\hline$x+166$ & $x+$ & 166 & 19392.49 & 19392.55 & 0.061037 & 3.147454 \\
\hline
\end{tabular}


Table S3D. Summary of UVPD mass spectrum for G12S•GDP complex: fragment ions incorporating mass shift of +463.9859 Da (GDP)

\begin{tabular}{|c|c|c|c|c|c|c|}
\hline & $\underline{\text { Ion }}$ & & Theoretical & Experimental & & $\mathrm{ppm}$ \\
\hline Ion & Type & Residue & $\underline{\text { Mass }}$ & Mass & $\Delta$ mass & error \\
\hline a27 & a & 27 & 3193.501 & 3193.529 & 0.028031 & 8.777515 \\
\hline b123 & $b$ & 123 & 14319.77 & 14319.86 & 0.093487 & 6.528528 \\
\hline 124 & $b$ & 124 & 14475.87 & 14475.87 & 0.000678 & 0.046837 \\
\hline$a+125$ & $a+$ & 125 & 14549.93 & 14549.93 & -0.00028 & -0.01938 \\
\hline b143 & $b$ & 143 & 16579.96 & 16579.96 & -0.0069 & -0.41604 \\
\hline a144 & a & 144 & 16681.01 & 16680.95 & -0.06476 & -3.8822 \\
\hline c144 & c & 144 & 16726.03 & 16725.98 & -0.04991 & -2.98391 \\
\hline b147 & $b$ & 147 & 16968.12 & 16968.12 & -0.00615 & -0.36256 \\
\hline c148 & c & 148 & 17113.24 & 17113.1 & -0.14743 & -8.61502 \\
\hline$a+154$ & $a+$ & 154 & 17725.55 & 17725.52 & -0.03801 & -2.14425 \\
\hline b154 & $b$ & 154 & 17752.54 & 17752.5 & -0.03722 & -2.09677 \\
\hline c157 & c & 157 & 18102.7 & 18102.71 & 0.006084 & 0.336082 \\
\hline$a+159$ & $a+$ & 159 & 18322.8 & 18322.83 & 0.035905 & 1.959579 \\
\hline$a+160$ & $a+$ & 160 & 18435.88 & 18435.91 & 0.024106 & 1.307557 \\
\hline c161 & $c$ & 161 & 18578.96 & 18579.01 & 0.046803 & 2.519139 \\
\hline$a+163$ & $a+$ & 163 & 18820.09 & 18820.11 & 0.017247 & 0.916412 \\
\hline a164 & $a$ & 164 & 18932.17 & 18932.19 & 0.022387 & 1.182485 \\
\hline$a+165$ & $a+$ & 165 & 19089.28 & 19089.37 & 0.087552 & 4.586447 \\
\hline a166 & $a$ & 166 & 19216.37 & 19216.35 & -0.01599 & -0.832 \\
\hline a167 & a & 167 & 19353.43 & 19353.55 & 0.120237 & 6.212699 \\
\hline z42 & $z$ & 42 & 5340.572 & 5340.586 & 0.013544 & 2.536058 \\
\hline z43 & z & 43 & 5441.62 & 5441.619 & -0.0005 & -0.09244 \\
\hline y43 & y & 43 & 5457.638 & 5457.641 & 0.002763 & 0.506263 \\
\hline$x+52$ & $x+$ & 52 & 6471.075 & 6471.097 & 0.022608 & 3.493696 \\
\hline$x+53$ & $x+$ & 53 & 6599.17 & 6599.191 & 0.02091 & 3.168576 \\
\hline$y-64$ & $y-$ & 64 & 7711.723 & 7711.769 & 0.045628 & 5.91671 \\
\hline$x+86$ & $x+$ & 86 & 10491.14 & 10491.12 & -0.01816 & -1.7308 \\
\hline$x+99$ & $x+$ & 99 & 11965.81 & 11965.75 & -0.05818 & -4.86202 \\
\hline$x+100$ & $x+$ & 100 & 12093.87 & 12093.87 & -0.00348 & -0.2875 \\
\hline$x+101$ & $x+$ & 101 & 12208.9 & 12208.88 & -0.01545 & -1.26531 \\
\hline x117 & $x$ & 117 & 14000.72 & 14000.71 & -0.0072 & -0.51447 \\
\hline$x+118$ & $x+$ & 118 & 14114.81 & 14114.8 & -0.01561 & -1.10622 \\
\hline$y-119$ & $y-$ & 119 & 14189.83 & 14189.8 & -0.02989 & -2.10637 \\
\hline$y-121$ & $y-$ & 121 & 14419.92 & 14419.89 & -0.02739 & -1.89918 \\
\hline z121 & $z$ & 121 & 14404.91 & 14404.93 & 0.021396 & 1.485327 \\
\hline$x 122$ & $x$ & 122 & 14503.93 & 14503.94 & 0.011916 & 0.821571 \\
\hline$x+123$ & $x+$ & 123 & 14619.96 & 14619.99 & 0.031838 & 2.177706 \\
\hline
\end{tabular}




\begin{tabular}{|c|c|c|c|c|c|c|}
\hline y124 & y & 124 & 14706.06 & 14705.93 & -0.12641 & -8.59585 \\
\hline y125 & y & 125 & 14805.13 & 14805.1 & -0.0249 & -1.68178 \\
\hline$x+125$ & $x+$ & 125 & 14832.11 & 14832.11 & -0.00393 & -0.26517 \\
\hline$x+126$ & $x+$ & 126 & 14931.18 & 14931.08 & -0.10384 & -6.95438 \\
\hline$y-127$ & $y-$ & 127 & 15031.24 & 15031.21 & -0.03933 & -2.61641 \\
\hline$x+127$ & $x+$ & 127 & 15059.24 & 15059.25 & 0.00897 & 0.595645 \\
\hline y128 & $y$ & 128 & 15160.35 & 15160.33 & -0.01736 & -1.14489 \\
\hline$x+128$ & $x+$ & 128 & 15187.33 & 15187.32 & -0.01104 & -0.72686 \\
\hline$x+129$ & $x+$ & 129 & 15343.44 & 15343.4 & -0.03471 & -2.26214 \\
\hline z129 & $z$ & 129 & 15300.43 & 15300.41 & -0.02158 & -1.41022 \\
\hline$y-129$ & $y-$ & 129 & 15315.44 & 15315.43 & -0.01437 & -0.9384 \\
\hline x130 & $x$ & 130 & 15505.49 & 15505.45 & -0.04387 & -2.82964 \\
\hline y132 & y & 132 & 15681.57 & 15681.54 & -0.03406 & -2.17223 \\
\hline$y-133$ & $y^{-}$ & 133 & 15809.61 & 15809.58 & -0.02841 & -1.79726 \\
\hline x133 & $x$ & 133 & 15836.59 & 15836.57 & -0.02009 & -1.26883 \\
\hline$x+133$ & $x+$ & 133 & 15837.6 & 15837.58 & -0.01553 & -0.98064 \\
\hline$x+135$ & $x+$ & 135 & 16051.73 & 16051.78 & 0.045126 & 2.811283 \\
\hline$y-136$ & $y^{-}$ & 136 & 16120.79 & 16120.77 & -0.02151 & -1.33418 \\
\hline$x+139$ & $x+$ & 139 & 16555.92 & 16555.91 & -0.01012 & -0.61144 \\
\hline z139 & $z$ & 139 & 16512.91 & 16512.93 & 0.014287 & 0.865202 \\
\hline x140 & $x$ & 140 & 16669.94 & 16669.94 & 0.005918 & 0.35501 \\
\hline$y-141$ & $y-$ & 141 & 16742.02 & 16742.01 & -0.01095 & -0.65398 \\
\hline$x+141$ & $x+$ & 141 & 16770.01 & 16770 & -0.00977 & -0.58259 \\
\hline y142 & y & 142 & 16890.09 & 16890.12 & 0.026098 & 1.545166 \\
\hline x144 & $x$ & 144 & 17167.18 & 17167.13 & -0.04426 & -2.57847 \\
\hline x145 & $x$ & 145 & 17295.23 & 17295.22 & -0.01301 & -0.7524 \\
\hline$y-147$ & $y-$ & 147 & 17494.42 & 17494.38 & -0.03725 & -2.12902 \\
\hline z147 & $z$ & 147 & 17479.4 & 17479.4 & -0.00214 & -0.1222 \\
\hline x148 & $x$ & 148 & 17649.46 & 17649.45 & -0.00967 & -0.54806 \\
\hline x149 & $x$ & 149 & 17762.54 & 17762.53 & -0.01365 & -0.76875 \\
\hline z150 & $z$ & 150 & 17821.59 & 17821.6 & 0.002613 & 0.14662 \\
\hline y150 & $y$ & 150 & 17837.61 & 17837.63 & 0.01583 & 0.887451 \\
\hline x150 & $x$ & 150 & 17863.59 & 17863.66 & 0.064397 & 3.60493 \\
\hline$x 151$ & $x$ & 151 & 17976.68 & 17976.66 & -0.01991 & -1.10738 \\
\hline z151 & z & 151 & 17934.68 & 17934.68 & 0.005467 & 0.304828 \\
\hline y152 & y & 152 & 18021.73 & 18021.7 & -0.03405 & -1.88944 \\
\hline x154 & $x$ & 154 & 18262.84 & 18262.86 & 0.014479 & 0.792812 \\
\hline$x+157$ & $x+$ & 157 & 18476.96 & 18476.93 & -0.03388 & -1.83337 \\
\hline y157 & y & 157 & 18449.97 & 18449.94 & -0.02823 & -1.53008 \\
\hline$y-158$ & $y-$ & 158 & 18536 & 18535.99 & -0.00849 & -0.45803 \\
\hline y161 & $y$ & 161 & 18764.13 & 18764.11 & -0.02293 & -1.22217 \\
\hline$y-162$ & $y-$ & 162 & 18862.19 & 18862.16 & -0.03567 & -1.89092 \\
\hline$x+162$ & $x+$ & 162 & 18890.19 & 18890.17 & -0.0152 & -0.80471 \\
\hline
\end{tabular}




$\begin{array}{lllllll}\text { x163 } & x & 163 & 18988.25 & 18988.24 & -0.00493 & -0.25958 \\ y-165 & y- & 165 & 19202.44 & 19202.35 & -0.08912 & -4.64097 \\ \text { x166 } & x & 166 & 19392.49 & 19392.55 & 0.061062 & 3.148745\end{array}$




\section{Table S4A. Summary of UVPD mass spectrum for G12S•GDPnP complex: apo fragment ions}

Column "Ions" is the fragment ion identity $(a, b, c, x, y, z)$ along with the number of residues in the fragment ion. A plus or minus sign indicates the fragment ion has one additional hydrogen atom or one less hydrogen atom than a conventional fragment ion. Column "Ion Type" is the type of fragment ion $(a, b, c, x, y, z)$. A plus sign indicates a fragment ion $(a, x)$ with an additional hydrogen atom; a minus sign indicates a fragment ion ( $y$ ) that is missing one hydrogen atom (the addition of one hydrogen atom for $a / x$ ions is not uncommon in UVPD mass spectra of intact proteins); Column "Residue" is the number of residues in the fragment ion. Column "Theoretical Mass" is the theoretical mass of the fragment ion; Column "Experimental Mass" is the experimentally measured mass of the fragment ion. Column "Delta mass" is the mass difference between the theoretical and experimental masses; and column "ppm error" is the mass error in ppm. The "apo" table (Table S4A) provides the fragment ions that do not retain the ligand. Tables S4B, S4C, and S4D provide the fragment ions that incorporate the ligand based on the mass shift corresponding to the retained ligand (with or without an additional hydrogen atom).

\begin{tabular}{|c|c|c|c|c|c|c|}
\hline Ion & $\frac{\text { Ion }}{\text { Type }}$ & Residue & $\begin{array}{l}\text { Theoretical } \\
\text { Mass }\end{array}$ & $\begin{array}{l}\text { Experimental } \\
\text { Mass }\end{array}$ & $\Delta$ mass & $\begin{array}{l}\text { ppm } \\
\text { error }\end{array}$ \\
\hline$\overline{a+4}$ & $\overline{a+}$ & 4 & 391.1651 & 391.1667 & 0.001544 & 3.9471 \\
\hline a4 & $\mathrm{a}$ & 4 & 390.1573 & 390.1589 & 0.001556 & 3.988135 \\
\hline b4 & $b$ & 4 & 418.1522 & 418.1538 & 0.001606 & 3.840707 \\
\hline c4 & c & 4 & 435.1785 & 435.1803 & 0.001825 & 4.193681 \\
\hline a5 & $a$ & 5 & 553.2206 & 553.2227 & 0.002069 & 3.739918 \\
\hline b5 & $b$ & 5 & 581.2156 & 581.2178 & 0.002271 & 3.907328 \\
\hline$a+5$ & $a+$ & 5 & 554.2285 & 554.2309 & 0.002423 & 4.371785 \\
\hline c5 & c & 5 & 598.2419 & 598.2444 & 0.002521 & 4.214015 \\
\hline$a+6$ & $a+$ & 6 & 682.3234 & 682.3262 & 0.002799 & 4.102113 \\
\hline b7 & $b$ & 7 & 822.3946 & 822.3981 & 0.003488 & 4.241273 \\
\hline$a+7$ & $a+$ & 7 & 795.4075 & 795.4111 & 0.003639 & 4.574973 \\
\hline a8 & $\mathrm{a}$ & 8 & 893.4681 & 893.4718 & 0.003723 & 4.166909 \\
\hline b8 & $b$ & 8 & 921.463 & 921.4669 & 0.003925 & 4.259531 \\
\hline$a+8$ & $a+$ & 8 & 894.4759 & 894.4801 & 0.004199 & 4.694333 \\
\hline c8 & $c$ & 8 & 938.4893 & 938.4939 & 0.004603 & 4.904691 \\
\hline$a+9$ & $a+$ & 9 & 993.5443 & 993.5486 & 0.004332 & 4.360116 \\
\hline b9 & $b$ & 9 & 1020.531 & 1020.536 & 0.004363 & 4.275224 \\
\hline c9 & c & 9 & 1037.558 & 1037.562 & 0.004491 & 4.328434 \\
\hline b10 & $b$ & 10 & 1119.6 & 1119.604 & 0.004374 & 3.906753 \\
\hline a10 & $a$ & 10 & 1091.605 & 1091.609 & 0.004538 & 4.157182 \\
\hline c10 & c & 10 & 1136.626 & 1136.631 & 0.004685 & 4.121848 \\
\hline$a+11$ & $a+$ & 11 & 1149.634 & 1149.639 & 0.004672 & 4.063874 \\
\hline b11 & $b$ & 11 & 1176.621 & 1176.626 & 0.004886 & 4.152568 \\
\hline c11 & c & 11 & 1193.648 & 1193.653 & 0.005808 & 4.865758 \\
\hline$a+12$ & $a+$ & 12 & 1220.671 & 1220.676 & 0.004915 & 4.026447 \\
\hline c12 & c & 12 & 1264.685 & 1264.69 & 0.004953 & 3.916391 \\
\hline b12 & $b$ & 12 & 1247.658 & 1247.664 & 0.00513 & 4.111702 \\
\hline
\end{tabular}




\begin{tabular}{|c|c|c|c|c|c|c|}
\hline b13 & $b$ & 13 & 1334.69 & 1334.694 & 0.003739 & 2.801399 \\
\hline c13 & c & 13 & 1351.717 & 1351.721 & 0.004783 & 3.538463 \\
\hline a13 & $a$ & 13 & 1306.695 & 1306.701 & 0.00549 & 4.201438 \\
\hline$a+13$ & $a+$ & 13 & 1307.703 & 1307.709 & 0.005722 & 4.375586 \\
\hline$a+14$ & $a+$ & 14 & 1364.725 & 1364.73 & 0.00477 & 3.495187 \\
\hline b14 & $b$ & 14 & 1391.712 & 1391.717 & 0.005107 & 3.669581 \\
\hline c14 & c & 14 & 1408.738 & 1408.744 & 0.005418 & 3.845995 \\
\hline$a+15$ & $a+$ & 15 & 1463.793 & 1463.798 & 0.004719 & 3.223794 \\
\hline a15 & $\mathrm{a}$ & 15 & 1462.785 & 1462.791 & 0.005708 & 3.902145 \\
\hline b15 & $b$ & 15 & 1490.78 & 1490.786 & 0.006032 & 4.046203 \\
\hline$a+16$ & $a+$ & 16 & 1520.815 & 1520.82 & 0.005476 & 3.600681 \\
\hline c16 & c & 16 & 1564.828 & 1564.834 & 0.006246 & 3.991493 \\
\hline b16 & $b$ & 16 & 1547.802 & 1547.809 & 0.006789 & 4.386221 \\
\hline$a+17$ & $a+$ & 17 & 1648.91 & 1648.916 & 0.006511 & 3.948651 \\
\hline c17 & c & 17 & 1692.923 & 1692.93 & 0.006525 & 3.85428 \\
\hline a17 & $a$ & 17 & 1647.902 & 1647.909 & 0.007208 & 4.374047 \\
\hline$a+18$ & $a+$ & 18 & 1735.942 & 1735.947 & 0.005683 & 3.273709 \\
\hline$a+19$ & $a+$ & 19 & 1806.979 & 1806.985 & 0.005927 & 3.280043 \\
\hline b19 & $b$ & 19 & 1833.966 & 1833.973 & 0.007532 & 4.106947 \\
\hline b20 & $b$ & 20 & 1947.05 & 1947.046 & -0.00358 & -1.83765 \\
\hline$a+20$ & $a+$ & 20 & 1920.063 & 1920.07 & 0.007072 & 3.683196 \\
\hline c20 & c & 20 & 1964.076 & 1964.084 & 0.007561 & 3.849647 \\
\hline a21 & a & 21 & 2020.103 & 2020.111 & 0.007914 & 3.917623 \\
\hline$a+22$ & $a+$ & 22 & 2134.195 & 2134.201 & 0.006079 & 2.848366 \\
\hline c22 & $c$ & 22 & 2178.208 & 2178.216 & 0.008474 & 3.890354 \\
\hline$a+23$ & $a+$ & 23 & 2262.253 & 2262.258 & 0.004496 & 1.987385 \\
\hline b23 & $b$ & 23 & 2289.24 & 2289.25 & 0.009397 & 4.104855 \\
\hline$a+24$ & $a+$ & 24 & 2375.337 & 2375.343 & 0.005933 & 2.497737 \\
\hline b24 & $b$ & 24 & 2402.324 & 2402.333 & 0.008833 & 3.676856 \\
\hline$a+25$ & $a+$ & 25 & 2488.421 & 2488.428 & 0.006834 & 2.746307 \\
\hline b25 & $b$ & 25 & 2515.408 & 2515.417 & 0.009001 & 3.578345 \\
\hline c25 & c & 25 & 2532.435 & 2532.444 & 0.009801 & 3.870189 \\
\hline$a+26$ & $a+$ & 26 & 2616.48 & 2616.486 & 0.006115 & 2.337097 \\
\hline c26 & c & 26 & 2660.493 & 2660.502 & 0.008838 & 3.32194 \\
\hline a26 & a & 26 & 2615.472 & 2615.482 & 0.009545 & 3.649437 \\
\hline$a+27$ & $a+$ & 27 & 2730.523 & 2730.53 & 0.006886 & 2.52185 \\
\hline a27 & $a$ & 27 & 2729.515 & 2729.524 & 0.00934 & 3.421853 \\
\hline c27 & c & 27 & 2774.536 & 2774.546 & 0.010342 & 3.72747 \\
\hline c28 & c & 28 & 2911.595 & 2911.574 & -0.02127 & -7.30596 \\
\hline$a+28$ & $a+$ & 28 & 2867.582 & 2867.59 & 0.008767 & 3.057269 \\
\hline b28 & $b$ & 28 & 2894.569 & 2894.581 & 0.012156 & 4.199589 \\
\hline a28 & a & 28 & 2866.574 & 2866.586 & 0.012367 & 4.314209 \\
\hline c29 & c & 29 & 3058.663 & 3058.639 & -0.02416 & -7.89789 \\
\hline
\end{tabular}




\begin{tabular}{|c|c|c|c|c|c|c|}
\hline$a+29$ & $a+$ & 29 & 3014.65 & 3014.659 & 0.008961 & 2.972474 \\
\hline a29 & $a$ & 29 & 3013.642 & 3013.655 & 0.012256 & 4.06684 \\
\hline b29 & $b$ & 29 & 3041.637 & 3041.654 & 0.016469 & 5.414518 \\
\hline$a+30$ & $a+$ & 30 & 3113.718 & 3113.727 & 0.008666 & 2.783157 \\
\hline a30 & a & 30 & 3112.711 & 3112.723 & 0.012144 & 3.901423 \\
\hline$a+31$ & $a+$ & 31 & 3228.745 & 3228.755 & 0.009505 & 2.943858 \\
\hline a31 & $\mathrm{a}$ & 31 & 3227.738 & 3227.75 & 0.012792 & 3.963147 \\
\hline a32 & a & 32 & 3356.78 & 3356.794 & 0.014197 & 4.229351 \\
\hline c32 & c & 32 & 3401.801 & 3401.817 & 0.01559 & 4.582866 \\
\hline c33 & c & 33 & 3564.865 & 3564.873 & 0.008412 & 2.359697 \\
\hline$a+33$ & $a+$ & 33 & 3520.851 & 3520.861 & 0.010083 & 2.863787 \\
\hline a33 & $\mathrm{a}$ & 33 & 3519.844 & 3519.856 & 0.012829 & 3.644764 \\
\hline$a+34$ & $a+$ & 34 & 3635.878 & 3635.889 & 0.010975 & 3.018519 \\
\hline$a+35$ & $a+$ & 35 & 3732.931 & 3732.943 & 0.011685 & 3.130239 \\
\hline c35 & c & 35 & 3776.944 & 3776.958 & 0.013917 & 3.684725 \\
\hline a36 & a & 36 & 3832.971 & 3832.981 & 0.010449 & 2.726084 \\
\hline a37 & $a$ & 37 & 3946.055 & 3946.072 & 0.017406 & 4.410988 \\
\hline a38 & a & 38 & 4075.098 & 4075.111 & 0.013146 & 3.225935 \\
\hline$a+39$ & $a+$ & 39 & 4191.132 & 4191.143 & 0.010363 & 2.472594 \\
\hline b39 & $b$ & 39 & 4218.119 & 4218.133 & 0.013996 & 3.318066 \\
\hline$a+40$ & $a+$ & 40 & 4278.164 & 4278.176 & 0.011536 & 2.696476 \\
\hline c40 & c & 40 & 4322.178 & 4322.204 & 0.025978 & 6.010396 \\
\hline b40 & $b$ & 40 & 4305.151 & 4305.178 & 0.026325 & 6.114767 \\
\hline c41 & c & 41 & 4485.241 & 4485.253 & 0.011965 & 2.667638 \\
\hline a41 & $a$ & 41 & 4440.22 & 4440.235 & 0.015602 & 3.51379 \\
\hline$a+42$ & $a+$ & 42 & 4597.329 & 4597.344 & 0.015527 & 3.377389 \\
\hline a42 & $a$ & 42 & 4596.321 & 4596.337 & 0.016468 & 3.582866 \\
\hline$c 42$ & c & 42 & 4641.342 & 4641.359 & 0.017226 & 3.711426 \\
\hline$a+43$ & $a+$ & 43 & 4725.424 & 4725.438 & 0.014751 & 3.121618 \\
\hline b43 & $b$ & 43 & 4752.411 & 4752.428 & 0.017212 & 3.621741 \\
\hline c43 & c & 43 & 4769.437 & 4769.46 & 0.023218 & 4.86808 \\
\hline c44 & c & 44 & 4897.496 & 4897.5 & 0.004067 & 0.830424 \\
\hline$a+44$ & $a+$ & 44 & 4853.482 & 4853.496 & 0.013917 & 2.867419 \\
\hline$a+45$ & $a+$ & 45 & 4952.551 & 4952.567 & 0.016185 & 3.268006 \\
\hline b45 & b & 45 & 4979.538 & 4979.564 & 0.025799 & 5.181003 \\
\hline$a+46$ & $a+$ & 46 & 5051.619 & 5051.635 & 0.015524 & 3.073068 \\
\hline c46 & c & 46 & 5095.633 & 5095.652 & 0.01902 & 3.732608 \\
\hline a47 & a & 47 & 5163.695 & 5163.714 & 0.018147 & 3.514344 \\
\hline c47 & c & 47 & 5208.717 & 5208.74 & 0.022933 & 4.402812 \\
\hline a49 & a & 49 & 5335.744 & 5335.76 & 0.016378 & 3.069488 \\
\hline$a+50$ & $a+$ & 50 & 5465.794 & 5465.809 & 0.014791 & 2.706097 \\
\hline c50 & c & 50 & 5509.808 & 5509.84 & 0.032407 & 5.881694 \\
\hline$a+51$ & $a+$ & 51 & 5566.842 & 5566.859 & 0.017159 & 3.082352 \\
\hline
\end{tabular}




\begin{tabular}{|c|c|c|c|c|c|c|}
\hline c51 & c & 51 & 5610.855 & 5610.874 & 0.018662 & 3.326053 \\
\hline a52 & a & 52 & 5668.843 & 5668.858 & 0.015062 & 2.656979 \\
\hline b52 & $b$ & 52 & 5696.838 & 5696.859 & 0.020635 & 3.622185 \\
\hline a53 & a & 53 & 5781.927 & 5781.952 & 0.024264 & 4.196525 \\
\hline a54 & a & 54 & 5895.011 & 5895.025 & 0.01369 & 2.322303 \\
\hline a55 & a & 55 & 6010.038 & 6010.049 & 0.01031 & 1.715463 \\
\hline a56 & a & 56 & 6123.122 & 6123.141 & 0.018779 & 3.066899 \\
\hline a57 & $a$ & 57 & 6236.206 & 6236.225 & 0.018093 & 2.901283 \\
\hline c58 & c & 58 & 6396.255 & 6396.267 & 0.012174 & 1.903301 \\
\hline c59 & c & 59 & 6497.302 & 6497.321 & 0.018917 & 2.911516 \\
\hline$a+59$ & $a+$ & 59 & 6453.289 & 6453.313 & 0.024425 & 3.784887 \\
\hline a59 & $a$ & 59 & 6452.281 & 6452.31 & 0.028911 & 4.480741 \\
\hline b59 & $b$ & 59 & 6480.276 & 6480.334 & 0.058177 & 8.97755 \\
\hline a60 & a & 60 & 6523.318 & 6523.328 & 0.009879 & 1.514413 \\
\hline$a+61$ & $a+$ & 61 & 6581.347 & 6581.378 & 0.030154 & 4.581732 \\
\hline$a+62$ & $a+$ & 62 & 6709.406 & 6709.431 & 0.025285 & 3.768585 \\
\hline a63 & $a$ & 63 & 6837.441 & 6837.469 & 0.028654 & 4.190749 \\
\hline$a+64$ & $a+$ & 64 & 6967.491 & 6967.505 & 0.01408 & 2.020809 \\
\hline a65 & $\mathrm{a}$ & 65 & 7129.547 & 7129.574 & 0.027739 & 3.89071 \\
\hline c69 & c & 69 & 7619.779 & 7619.8 & 0.020929 & 2.746668 \\
\hline a69 & a & 69 & 7574.757 & 7574.786 & 0.028888 & 3.813719 \\
\hline b69 & $b$ & 69 & 7602.752 & 7602.782 & 0.029529 & 3.883988 \\
\hline$a+70$ & $a+$ & 70 & 7690.792 & 7690.817 & 0.024397 & 3.172231 \\
\hline$a+71$ & $a+$ & 71 & 7818.851 & 7818.874 & 0.023434 & 2.997112 \\
\hline b71 & $b$ & 71 & 7845.838 & 7845.868 & 0.030404 & 3.875176 \\
\hline c72 & c & 72 & 8025.928 & 8025.918 & -0.00934 & -1.16385 \\
\hline a72 & a & 72 & 7980.906 & 7980.928 & 0.02164 & 2.711472 \\
\hline a73 & $a$ & 73 & 8111.947 & 8111.976 & 0.02949 & 3.635379 \\
\hline c74 & c & 74 & 8313.069 & 8313.081 & 0.011582 & 1.393228 \\
\hline$a+74$ & $a+$ & 74 & 8269.056 & 8269.084 & 0.02839 & 3.433278 \\
\hline a75 & $a$ & 75 & 8369.096 & 8369.121 & 0.025767 & 3.078827 \\
\hline a77 & $a$ & 77 & 8555.16 & 8555.194 & 0.034104 & 3.986366 \\
\hline$a+78$ & $a+$ & 78 & 8613.189 & 8613.213 & 0.024229 & 2.813008 \\
\hline a79 & $a$ & 79 & 8759.249 & 8759.288 & 0.038473 & 4.392271 \\
\hline c80 & c & 80 & 8917.355 & 8917.382 & 0.027401 & 3.072772 \\
\hline a80 & a & 80 & 8872.334 & 8872.376 & 0.042792 & 4.823083 \\
\hline a82 & $a$ & 82 & 9074.411 & 9074.449 & 0.037945 & 4.181539 \\
\hline a83 & a & 83 & 9221.48 & 9221.529 & 0.049003 & 5.314006 \\
\hline a84 & $a$ & 84 & 9292.517 & 9292.527 & 0.010428 & 1.122193 \\
\hline c84 & c & 84 & 9337.538 & 9337.584 & 0.046586 & 4.98911 \\
\hline a85 & $a$ & 85 & 9405.601 & 9405.633 & 0.031837 & 3.384898 \\
\hline$a+86$ & $a+$ & 86 & 9520.651 & 9520.68 & 0.028625 & 3.006619 \\
\hline a87 & a & 87 & 9633.687 & 9633.718 & 0.031304 & 3.249431 \\
\hline
\end{tabular}




\begin{tabular}{|c|c|c|c|c|c|c|}
\hline c87 & c & 87 & 9678.708 & 9678.744 & 0.036334 & 3.754014 \\
\hline a88 & a & 88 & 9734.734 & 9734.791 & 0.056622 & 5.816492 \\
\hline a89 & a & 89 & 9862.829 & 9862.855 & 0.026238 & 2.660291 \\
\hline b89 & $b$ & 89 & 9890.824 & 9890.853 & 0.028693 & 2.900972 \\
\hline$a+90$ & $a+$ & 90 & 9950.869 & 9950.894 & 0.025323 & 2.5448 \\
\hline a91 & $a$ & 91 & 10096.93 & 10096.97 & 0.042619 & 4.220986 \\
\hline a92 & $a$ & 92 & 10225.97 & 10226 & 0.024077 & 2.354495 \\
\hline c92 & c & 92 & 10270.99 & 10271.02 & 0.029027 & 2.826114 \\
\hline a94 & a & 94 & 10454.08 & 10454.12 & 0.037903 & 3.625665 \\
\hline c96 & c & 96 & 10773.22 & 10773.25 & 0.027603 & 2.562186 \\
\hline a96 & a & 96 & 10728.2 & 10728.24 & 0.037271 & 3.474115 \\
\hline a97 & a & 97 & 10891.26 & 10891.3 & 0.032779 & 3.00966 \\
\hline a98 & $a$ & 98 & 11047.37 & 11047.4 & 0.033097 & 2.995918 \\
\hline$a+108$ & $a+$ & 108 & 12261.02 & 12260.9 & -0.11554 & -9.42369 \\
\hline$a+134$ & $a+$ & 134 & 15084.45 & 15084.3 & -0.14466 & -9.58968 \\
\hline a163 & $a$ & 163 & 18355.1 & 18354.93 & -0.16669 & -9.08118 \\
\hline$a+169$ & $a+$ & 169 & 19147.58 & 19147.4 & -0.18131 & -9.46903 \\
\hline y3 & y & 3 & 403.2431 & 403.2446 & 0.00151 & 3.74464 \\
\hline $\mathrm{z} 4$ & z & 4 & 524.2832 & 524.2851 & 0.001862 & 3.551516 \\
\hline y4 & y & 4 & 540.302 & 540.304 & 0.002018 & 3.734948 \\
\hline$x 4$ & $x$ & 4 & 566.2813 & 566.2834 & 0.002123 & 3.74902 \\
\hline$x 6$ & $x$ & 6 & 850.4773 & 850.4808 & 0.003502 & 4.117688 \\
\hline y6 & y & 6 & 824.4981 & 824.5016 & 0.00358 & 4.342036 \\
\hline y7 & y & 7 & 937.5821 & 937.5862 & 0.004053 & 4.322821 \\
\hline$x 7$ & $x$ & 7 & 963.5614 & 963.5655 & 0.004097 & 4.251935 \\
\hline$z 7$ & z & 7 & 921.5634 & 921.5678 & 0.004447 & 4.825496 \\
\hline y8 & y & 8 & 1066.625 & 1066.629 & 0.004737 & 4.441112 \\
\hline$x+8$ & $x+$ & 8 & 1093.612 & 1093.622 & 0.009713 & 8.88155 \\
\hline$y-9$ & $y-$ & 9 & 1221.718 & 1221.722 & 0.003737 & 3.058834 \\
\hline y9 & $y$ & 9 & 1222.726 & 1222.731 & 0.00519 & 4.244615 \\
\hline$x 9$ & $x$ & 9 & 1248.705 & 1248.71 & 0.005417 & 4.338094 \\
\hline$x 10$ & $x$ & 10 & 1347.773 & 1347.779 & 0.005122 & 3.800342 \\
\hline y10 & y & 10 & 1321.794 & 1321.8 & 0.005749 & 4.349391 \\
\hline$x 11$ & $x$ & 11 & 1460.858 & 1460.863 & 0.005657 & 3.872383 \\
\hline y11 & y & 11 & 1434.878 & 1434.884 & 0.006162 & 4.294441 \\
\hline$x 12$ & $x$ & 12 & 1561.905 & 1561.912 & 0.006282 & 4.022011 \\
\hline y12 & y & 12 & 1535.926 & 1535.934 & 0.008287 & 5.395442 \\
\hline y13 & $y$ & 13 & 1698.989 & 1698.996 & 0.006768 & 3.983545 \\
\hline$x 13$ & $x$ & 13 & 1724.969 & 1724.976 & 0.007731 & 4.481821 \\
\hline$x+14$ & $x+$ & 14 & 1873.045 & 1873.048 & 0.002982 & 1.592043 \\
\hline z14 & $z$ & 14 & 1830.039 & 1830.044 & 0.005223 & 2.854038 \\
\hline y14 & y & 14 & 1846.058 & 1846.068 & 0.009895 & 5.360071 \\
\hline x15 & $x$ & 15 & 1943.074 & 1943.056 & -0.01832 & -9.42887 \\
\hline
\end{tabular}




\begin{tabular}{|c|c|c|c|c|c|c|}
\hline y15 & y & 15 & 1917.095 & 1917.103 & 0.007697 & 4.014929 \\
\hline$x+16$ & $x+$ & 16 & 2059.109 & 2059.113 & 0.004043 & 1.963455 \\
\hline y16 & y & 16 & 2032.122 & 2032.13 & 0.007857 & 3.866402 \\
\hline y17 & y & 17 & 2147.149 & 2147.157 & 0.008138 & 3.790143 \\
\hline y19 & y & 19 & 2303.239 & 2303.247 & 0.008845 & 3.840245 \\
\hline z20 & $z$ & 20 & 2415.278 & 2415.285 & 0.007116 & 2.946244 \\
\hline y20 & $y$ & 20 & 2431.297 & 2431.305 & 0.007638 & 3.141533 \\
\hline y21 & $y$ & 21 & 2587.398 & 2587.416 & 0.017368 & 6.712534 \\
\hline y22 & y & 22 & 2688.446 & 2688.456 & 0.009923 & 3.69098 \\
\hline$y-24$ & $y-$ & 24 & 2886.57 & 2886.554 & -0.01667 & -5.77362 \\
\hline z25 & $z$ & 25 & 2958.591 & 2958.598 & 0.00697 & 2.355851 \\
\hline y25 & y & 25 & 2974.61 & 2974.622 & 0.012083 & 4.062045 \\
\hline$x+26$ & $x+$ & 26 & 3102.645 & 3102.655 & 0.010123 & 3.26269 \\
\hline y26 & y & 26 & 3075.658 & 3075.67 & 0.012546 & 4.079128 \\
\hline y27 & y & 27 & 3204.7 & 3204.711 & 0.010653 & 3.32418 \\
\hline$y-28$ & $y-$ & 28 & 3316.777 & 3316.787 & 0.010346 & 3.119303 \\
\hline y28 & $y$ & 28 & 3317.784 & 3317.795 & 0.011066 & 3.335358 \\
\hline$x 28$ & $x$ & 28 & 3343.764 & 3343.777 & 0.013565 & 4.056806 \\
\hline y29 & y & 29 & 3464.853 & 3464.857 & 0.004498 & 1.298179 \\
\hline$x+29$ & $x+$ & 29 & 3491.84 & 3491.849 & 0.008666 & 2.481777 \\
\hline z29 & $z$ & 29 & 3448.834 & 3448.845 & 0.011103 & 3.219349 \\
\hline$x 29$ & $x$ & 29 & 3490.832 & 3490.846 & 0.014172 & 4.059777 \\
\hline z30 & $z$ & 30 & 3545.887 & 3545.855 & -0.03169 & -8.93824 \\
\hline$x+30$ & $x+$ & 30 & 3588.893 & 3588.885 & -0.00714 & -1.98809 \\
\hline$y-30$ & $y^{-}$ & 30 & 3560.898 & 3560.907 & 0.009049 & 2.541222 \\
\hline y30 & $y$ & 30 & 3561.906 & 3561.919 & 0.013038 & 3.6604 \\
\hline$y-31$ & $y-$ & 31 & 3673.982 & 3673.989 & 0.007583 & 2.063982 \\
\hline$x+31$ & $x+$ & 31 & 3701.977 & 3701.986 & 0.009542 & 2.577533 \\
\hline x31 & $x$ & 31 & 3700.969 & 3700.984 & 0.015047 & 4.065692 \\
\hline$x+32$ & $x+$ & 32 & 3758.998 & 3759.007 & 0.00903 & 2.402227 \\
\hline z32 & $z$ & 32 & 3715.992 & 3716.008 & 0.015709 & 4.227404 \\
\hline$y-32$ & $y-$ & 32 & 3731.003 & 3731.022 & 0.018378 & 4.925762 \\
\hline$y-33$ & $y^{-}$ & 33 & 3894.067 & 3894.074 & 0.007924 & 2.034899 \\
\hline y33 & $y$ & 33 & 3895.074 & 3895.085 & 0.010841 & 2.783259 \\
\hline y34 & $y$ & 34 & 3982.106 & 3982.119 & 0.012136 & 3.047633 \\
\hline$y-34$ & $y-$ & 34 & 3981.099 & 3981.119 & 0.020768 & 5.216659 \\
\hline$x+35$ & $x+$ & 35 & 4165.195 & 4165.199 & 0.004502 & 1.080854 \\
\hline$y-35$ & $y-$ & 35 & 4137.2 & 4137.221 & 0.021137 & 5.109019 \\
\hline$x+36$ & $x+$ & 36 & 4236.232 & 4236.24 & 0.008286 & 1.955976 \\
\hline$y-36$ & $y-$ & 36 & 4208.237 & 4208.248 & 0.010769 & 2.559037 \\
\hline$x+37$ & $x+$ & 37 & 4349.316 & 4349.327 & 0.010896 & 2.505214 \\
\hline$y-37$ & $y-$ & 37 & 4321.321 & 4321.333 & 0.012281 & 2.841963 \\
\hline z37 & $z$ & 37 & 4306.31 & 4306.324 & 0.013699 & 3.181146 \\
\hline
\end{tabular}




\begin{tabular}{|c|c|c|c|c|c|c|}
\hline$x+38$ & $x+$ & 38 & 4464.343 & 4464.359 & 0.016304 & 3.652042 \\
\hline$x+39$ & $x+$ & 39 & 4592.401 & 4592.41 & 0.008872 & 1.93188 \\
\hline y39 & $y$ & 39 & 4565.414 & 4565.428 & 0.01371 & 3.003013 \\
\hline$x+40$ & $x+$ & 40 & 4663.438 & 4663.449 & 0.010458 & 2.242544 \\
\hline$y-40$ & $y-$ & 40 & 4635.443 & 4635.465 & 0.0216 & 4.659755 \\
\hline y41 & y & 41 & 4764.51 & 4764.519 & 0.009159 & 1.922338 \\
\hline$x+41$ & $x+$ & 41 & 4791.497 & 4791.511 & 0.014378 & 3.000726 \\
\hline z41 & $z$ & 41 & 4748.491 & 4748.507 & 0.016172 & 3.405713 \\
\hline$y-41$ & $y-$ & 41 & 4763.502 & 4763.522 & 0.019896 & 4.176766 \\
\hline$x+42$ & $x+$ & 42 & 4919.592 & 4919.604 & 0.011948 & 2.42865 \\
\hline z43 & $z$ & 43 & 4977.634 & 4977.65 & 0.016387 & 3.292126 \\
\hline y43 & y & 43 & 4993.653 & 4993.671 & 0.018252 & 3.65504 \\
\hline$x+43$ & $x+$ & 43 & 5020.64 & 5020.664 & 0.024233 & 4.826669 \\
\hline y44 & y & 44 & 5108.679 & 5108.703 & 0.02353 & 4.605887 \\
\hline$y-45$ & $y-$ & 45 & 5206.74 & 5206.727 & -0.01308 & -2.51212 \\
\hline$x+45$ & $x+$ & 45 & 5234.735 & 5234.75 & 0.015402 & 2.942263 \\
\hline$y-46$ & $y-$ & 46 & 5307.788 & 5307.741 & -0.0464 & -8.74111 \\
\hline$x+46$ & $x+$ & 46 & 5335.783 & 5335.76 & -0.02251 & -4.21907 \\
\hline$x+47$ & $x+$ & 47 & 5491.884 & 5491.908 & 0.024512 & 4.463308 \\
\hline$x+48$ & $x+$ & 48 & 5578.916 & 5578.946 & 0.030186 & 5.410723 \\
\hline y49 & $y$ & 49 & 5648.981 & 5648.994 & 0.012368 & 2.189421 \\
\hline$x+49$ & $x+$ & 49 & 5675.969 & 5675.997 & 0.028228 & 4.973242 \\
\hline$x+50$ & $x+$ & 50 & 5789.053 & 5789.074 & 0.021481 & 3.710619 \\
\hline$x+51$ & $x+$ & 51 & 5904.08 & 5904.106 & 0.026035 & 4.409657 \\
\hline$y-52$ & $y^{-}$ & 52 & 5979.094 & 5979.121 & 0.026879 & 4.495503 \\
\hline$x+56$ & $x+$ & 56 & 6405.317 & 6405.339 & 0.02224 & 3.472111 \\
\hline$x+59$ & $x+$ & 59 & 6748.509 & 6748.532 & 0.022454 & 3.327248 \\
\hline$x+62$ & $x+$ & 62 & 7059.658 & 7059.694 & 0.036453 & 5.16356 \\
\hline y64 & $y$ & 64 & 7248.745 & 7248.77 & 0.025144 & 3.468738 \\
\hline
\end{tabular}


Table S4B. Summary of UVPD mass spectrum for G12S•GDPnP complex: fragment ions incorporating mass shift of +543.9760 Da (GDPnp)

\begin{tabular}{|c|c|c|c|c|c|c|}
\hline & $\underline{\text { Ion }}$ & & Theoretical & Experimental & & $\mathrm{ppm}$ \\
\hline Ion & Type & Residue & Mass & Mass & $\Delta$ mass & error \\
\hline c134 & c & 134 & 15672.43 & 15672.54 & 0.105665 & 6.742092 \\
\hline$a+135$ & $a+$ & 135 & 15699.46 & 15699.49 & 0.036327 & 2.313899 \\
\hline$a+136$ & $a+$ & 136 & 15855.56 & 15855.62 & 0.061926 & 3.905631 \\
\hline a137 & a & 137 & 15941.58 & 15941.61 & 0.024235 & 1.520238 \\
\hline$a+138$ & $a+$ & 138 & 16105.66 & 16105.72 & 0.063001 & 3.91173 \\
\hline a139 & a & 139 & 16161.67 & 16161.8 & 0.135792 & 8.402103 \\
\hline$a+140$ & $a+$ & 140 & 16275.76 & 16275.81 & 0.051476 & 3.162738 \\
\hline$a+142$ & $a+$ & 142 & 16519.88 & 16519.9 & 0.017245 & 1.043892 \\
\hline a144 & $\mathrm{a}$ & 144 & 16761 & 16760.97 & -0.03032 & -1.80914 \\
\hline c144 & c & 144 & 16806.02 & 16806.04 & 0.019561 & 1.163928 \\
\hline$a+154$ & $a+$ & 154 & 17805.54 & 17805.53 & -0.01235 & -0.69338 \\
\hline c161 & c & 161 & 18658.95 & 18659.01 & 0.05597 & 2.999632 \\
\hline b161 & $b$ & 161 & 18641.93 & 18642.04 & 0.108638 & 5.827616 \\
\hline$a+162$ & $a+$ & 162 & 18771.04 & 18771.04 & -0.00441 & -0.23472 \\
\hline$a+164$ & $a+$ & 164 & 19013.17 & 19013.32 & 0.148241 & 7.796753 \\
\hline$a+165$ & $a+$ & 165 & 19169.27 & 19169.46 & 0.190245 & 9.924477 \\
\hline b166 & $b$ & 166 & 19324.35 & 19324.41 & 0.05992 & 3.100751 \\
\hline$x 9$ & $x$ & 9 & 1792.681 & 1792.679 & -0.00195 & -1.09055 \\
\hline y46 & $y$ & 46 & 5852.772 & 5852.805 & 0.033208 & 5.673893 \\
\hline y47 & $y$ & 47 & 6008.873 & 6008.888 & 0.015105 & 2.513783 \\
\hline$x 47$ & $x$ & 47 & 6034.852 & 6034.906 & 0.054395 & 9.013477 \\
\hline$y-67$ & $y-$ & 67 & 8133.904 & 8133.983 & 0.0795 & 9.773909 \\
\hline y68 & $y$ & 68 & 8291.013 & 8291.084 & 0.071687 & 8.64635 \\
\hline y73 & y & 73 & 8945.394 & 8945.406 & 0.012517 & 1.399268 \\
\hline$x 78$ & $x$ & 78 & 9636.665 & 9636.671 & 0.006137 & 0.636839 \\
\hline$y-80$ & $y-$ & 80 & 9885.789 & 9885.852 & 0.062778 & 6.350331 \\
\hline$x+82$ & $x+$ & 82 & 10128.91 & 10128.91 & 0.001869 & 0.184518 \\
\hline$x+84$ & $x+$ & 84 & 10344 & 10344.01 & 0.005863 & 0.566799 \\
\hline$x 84$ & $x$ & 84 & 10342.99 & 10343.04 & 0.043497 & 4.205455 \\
\hline$y-86$ & $y-$ & 86 & 10543.13 & 10543.15 & 0.016732 & 1.587007 \\
\hline$x 86$ & $x$ & 86 & 10570.12 & 10570.15 & 0.02598 & 2.457872 \\
\hline$y-87$ & $y-$ & 87 & 10614.17 & 10614.17 & -0.00299 & -0.28217 \\
\hline$x+88$ & $x+$ & 88 & 10789.23 & 10789.24 & 0.0093 & 0.861967 \\
\hline$y-88$ & $y-$ & 88 & 10761.24 & 10761.25 & 0.013359 & 1.241403 \\
\hline$y-89$ & $y^{-}$ & 89 & 10860.31 & 10860.32 & 0.011762 & 1.083029 \\
\hline$x+89$ & $x+$ & 89 & 10888.3 & 10888.32 & 0.012993 & 1.193296 \\
\hline$y-90$ & $y^{-}$ & 90 & 10963.32 & 10963.33 & 0.009027 & 0.823385 \\
\hline x90 & $x$ & 90 & 10990.3 & 10990.32 & 0.017689 & 1.60951 \\
\hline
\end{tabular}




\begin{tabular}{|c|c|c|c|c|c|c|}
\hline$y-91$ & $y-$ & 91 & 11076.4 & 11076.4 & 0.000138 & 0.012462 \\
\hline x91 & $x$ & 91 & 11103.39 & 11103.39 & 0.002711 & 0.24416 \\
\hline$y-95$ & $y-$ & 95 & 11466.55 & 11466.56 & 0.004863 & 0.424106 \\
\hline$x+97$ & $x+$ & 97 & 11751.7 & 11751.68 & -0.02258 & -1.92168 \\
\hline$y-101$ & $y-$ & 101 & 12260.89 & 12260.9 & 0.011046 & 0.900916 \\
\hline$x+101$ & $x+$ & 101 & 12288.89 & 12288.9 & 0.015252 & 1.241119 \\
\hline z108 & $z$ & 108 & 13112.24 & 13112.22 & -0.02193 & -1.67264 \\
\hline y109 & y & 109 & 13256.32 & 13256.3 & -0.01421 & -1.07232 \\
\hline$y-110$ & $y-$ & 110 & 13312.33 & 13312.31 & -0.02502 & -1.87953 \\
\hline$x+110$ & $x+$ & 110 & 13340.33 & 13340.34 & 0.017661 & 1.323878 \\
\hline$y-113$ & $y-$ & 113 & 13599.44 & 13599.32 & -0.12057 & -8.86565 \\
\hline$x+113$ & $x+$ & 113 & 13627.44 & 13627.37 & -0.06929 & -5.08474 \\
\hline$x+116$ & $x+$ & 116 & 13968.63 & 13968.6 & -0.03515 & -2.5165 \\
\hline z116 & $z$ & 116 & 13925.63 & 13925.6 & -0.02786 & -2.00077 \\
\hline$x+118$ & $x+$ & 118 & 14194.8 & 14194.8 & 0.000389 & 0.027402 \\
\hline x119 & $x$ & 119 & 14296.8 & 14296.83 & 0.025391 & 1.775991 \\
\hline$x+120$ & $x+$ & 120 & 14398.86 & 14398.87 & 0.00846 & 0.587544 \\
\hline x121 & $x$ & 121 & 14526.89 & 14526.93 & 0.038881 & 2.676484 \\
\hline$x+122$ & $x+$ & 122 & 14584.92 & 14584.96 & 0.03694 & 2.53275 \\
\hline$x+123$ & $x+$ & 123 & 14699.95 & 14700.01 & 0.058828 & 4.001916 \\
\hline$y-124$ & $y^{-}$ & 124 & 14785.04 & 14785.08 & 0.042925 & 2.903275 \\
\hline x125 & $x$ & 125 & 14911.09 & 14911.11 & 0.017698 & 1.186901 \\
\hline$y-125$ & $y-$ & 125 & 14884.11 & 14884.18 & 0.069974 & 4.701258 \\
\hline x126 & $x$ & 126 & 15010.16 & 15010.23 & 0.069162 & 4.607678 \\
\hline$y-127$ & $y^{-}$ & 127 & 15111.23 & 15111.23 & -0.00745 & -0.49334 \\
\hline$x+127$ & $x+$ & 127 & 15139.23 & 15139.29 & 0.061594 & 4.068501 \\
\hline$y-128$ & $y-$ & 128 & 15239.33 & 15239.38 & 0.048219 & 3.164118 \\
\hline x128 & $x$ & 128 & 15266.32 & 15266.38 & 0.063082 & 4.132103 \\
\hline y129 & y & 129 & 15396.44 & 15396.51 & 0.074586 & 4.844367 \\
\hline x130 & $x$ & 130 & 15585.48 & 15585.51 & 0.033164 & 2.127878 \\
\hline x131 & $x$ & 131 & 15672.51 & 15672.54 & 0.027465 & 1.752431 \\
\hline y132 & $y$ & 132 & 15761.56 & 15761.57 & 0.013029 & 0.826631 \\
\hline x132 & $x$ & 132 & 15787.54 & 15787.63 & 0.08615 & 5.456835 \\
\hline y133 & $y$ & 133 & 15890.6 & 15890.61 & 0.008525 & 0.536481 \\
\hline x133 & $x$ & 133 & 15916.58 & 15916.64 & 0.054651 & 3.433589 \\
\hline x134 & $x$ & 134 & 16029.67 & 16029.57 & -0.09362 & -5.8403 \\
\hline$y-137$ & $y^{-}$ & 137 & 16315.81 & 16315.91 & 0.0984 & 6.030963 \\
\hline y138 & $y$ & 138 & 16479.88 & 16479.88 & -0.00058 & -0.03532 \\
\hline$x 138$ & $x$ & 138 & 16505.86 & 16505.91 & 0.056484 & 3.422058 \\
\hline$y-139$ & $y-$ & 139 & 16607.91 & 16607.96 & 0.04352 & 2.62044 \\
\hline x140 & $x$ & 140 & 16749.93 & 16750.01 & 0.079491 & 4.745752 \\
\hline$x+140$ & $x+$ & 140 & 16750.93 & 16751.02 & 0.084165 & 5.024494 \\
\hline x141 & $x$ & 141 & 16849 & 16849.02 & 0.026021 & 1.544365 \\
\hline
\end{tabular}




\begin{tabular}{|c|c|c|c|c|c|c|}
\hline$y-142$ & $y-$ & 142 & 16969.08 & 16969.08 & 0.00769 & 0.453179 \\
\hline$x+142$ & $x+$ & 142 & 16997.07 & 16997.13 & 0.058673 & 3.451946 \\
\hline$x+144$ & $x+$ & 144 & 17248.17 & 17248.23 & 0.055954 & 3.244052 \\
\hline x145 & $x$ & 145 & 17375.22 & 17375.25 & 0.029125 & 1.676237 \\
\hline z145 & $z$ & 145 & 17333.23 & 17333.3 & 0.07403 & 4.270988 \\
\hline$x+146$ & $x+$ & 146 & 17489.32 & 17489.41 & 0.091049 & 5.205976 \\
\hline$y-148$ & $y^{-}$ & 148 & 17702.46 & 17702.5 & 0.037074 & 2.094287 \\
\hline x148 & $x$ & 148 & 17729.45 & 17729.5 & 0.050764 & 2.863258 \\
\hline y149 & y & 149 & 17816.56 & 17816.58 & 0.02722 & 1.527793 \\
\hline x149 & $x$ & 149 & 17842.53 & 17842.58 & 0.041655 & 2.33459 \\
\hline$y-151$ & $y-$ & 151 & 18029.68 & 18029.72 & 0.044466 & 2.466269 \\
\hline y153 & $y$ & 153 & 18188.76 & 18188.8 & 0.046066 & 2.532664 \\
\hline$y-154$ & $y^{-}$ & 154 & 18315.84 & 18315.86 & 0.013911 & 0.759508 \\
\hline$x+154$ & $x+$ & 154 & 18343.84 & 18343.86 & 0.020705 & 1.128715 \\
\hline x155 & $x$ & 155 & 18399.85 & 18399.85 & -0.00147 & -0.08016 \\
\hline$y-155$ & $y-$ & 155 & 18372.87 & 18372.91 & 0.043476 & 2.366317 \\
\hline x156 & $x$ & 156 & 18498.92 & 18498.99 & 0.070007 & 3.784383 \\
\hline y158 & y & 158 & 18616.99 & 18617.01 & 0.016534 & 0.888113 \\
\hline$x+159$ & $x+$ & 159 & 18715.02 & 18715.02 & 0.000879 & 0.046966 \\
\hline$y-159$ & $y-$ & 159 & 18687.02 & 18687.03 & 0.00263 & 0.140741 \\
\hline x160 & $x$ & 160 & 18771.03 & 18771.04 & 0.004334 & 0.230888 \\
\hline z163 & z & 163 & 19026.24 & 19026.25 & 0.011207 & 0.589029 \\
\hline$x+163$ & $x+$ & 163 & 19069.25 & 19069.35 & 0.108174 & 5.672693 \\
\hline z166 & $z$ & 166 & 19430.48 & 19430.6 & 0.115867 & 5.963156 \\
\hline x166 & $x$ & 166 & 19472.48 & 19472.63 & 0.147134 & 7.555997 \\
\hline$y-168$ & $y-$ & 168 & 19675.58 & 19675.75 & 0.163094 & 8.289159 \\
\hline x168 & $x$ & 168 & 19702.57 & 19702.75 & 0.177957 & 9.032172 \\
\hline
\end{tabular}


Table S4C. Summary of UVPD mass spectrum for G12S•GDPnP complex: fragment ions incorporating mass shift of +541.9604 Da (GDPnp)

\begin{tabular}{|c|c|c|c|c|c|c|}
\hline & Ion & & Theoretical & Experimental & & ppm \\
\hline$\underline{\text { Ion }}$ & Type & $\underline{\text { Residue }}$ & $\underline{\text { Mass }}$ & $\underline{\text { Mass }}$ & $\underline{\Delta \text { mass }}$ & error \\
\hline c7 & c & 7 & 1381.381 & 1381.373 & -0.00818 & -5.91871 \\
\hline a7 & a & 7 & 1336.36 & 1336.367 & 0.007424 & 5.555389 \\
\hline b74 & $b$ & 74 & 8838.003 & 8838 & -0.0032 & -0.36207 \\
\hline b115 & $b$ & 115 & 13583.38 & 13583.43 & 0.051051 & 3.758344 \\
\hline$a+124$ & $a+$ & 124 & 14526.85 & 14526.93 & 0.077721 & 5.350158 \\
\hline b125 & $b$ & 125 & 14654.89 & 14654.99 & 0.103254 & 7.045703 \\
\hline$a+134$ & $a+$ & 134 & 15626.41 & 15626.51 & 0.102624 & 6.567343 \\
\hline c134 & c & 134 & 15670.42 & 15670.56 & 0.136438 & 8.706723 \\
\hline c135 & c & 135 & 15741.46 & 15741.55 & 0.095422 & 6.061828 \\
\hline$a+141$ & $a+$ & 141 & 16370.8 & 16370.85 & 0.055819 & 3.409667 \\
\hline c142 & c & 142 & 16561.88 & 16561.92 & 0.036146 & 2.182482 \\
\hline$a+145$ & $a+$ & 145 & 16861.04 & 16861.02 & -0.02091 & -1.24026 \\
\hline$a+149$ & $a+$ & 149 & 17248.25 & 17248.23 & -0.02292 & -1.32889 \\
\hline$a+151$ & $a+$ & 151 & 17532.41 & 17532.36 & -0.05024 & -2.86549 \\
\hline b155 & $b$ & 155 & 17945.54 & 17945.68 & 0.138209 & 7.701578 \\
\hline c158 & c & 158 & 18343.74 & 18343.86 & 0.12133 & 6.614246 \\
\hline a158 & $a$ & 158 & 18298.72 & 18298.87 & 0.15524 & 8.483655 \\
\hline a159 & a & 159 & 18399.76 & 18399.85 & 0.08632 & 4.691364 \\
\hline a160 & a & 160 & 18512.85 & 18513 & 0.147279 & 7.955502 \\
\hline b163 & b & 163 & 18925.06 & 18925.2 & 0.145577 & 7.692289 \\
\hline c164 & c & 164 & 19055.17 & 19055.26 & 0.092953 & 4.8781 \\
\hline$a+165$ & $a+$ & 165 & 19167.25 & 19167.39 & 0.141344 & 7.374242 \\
\hline$a+166$ & $a+$ & 166 & 19295.35 & 19295.44 & 0.09033 & 4.681437 \\
\hline$a+167$ & $a+$ & 167 & 19432.41 & 19432.51 & 0.107347 & 5.524121 \\
\hline y6 & y & 6 & 1366.458 & 1366.452 & -0.00638 & -4.66974 \\
\hline$x+72$ & $x+$ & 72 & 8814.264 & 8814.236 & -0.02823 & -3.20299 \\
\hline$y-73$ & $y-$ & 73 & 8942.37 & 8942.385 & 0.014471 & 1.618255 \\
\hline z73 & $z$ & 73 & 8927.359 & 8927.393 & 0.033346 & 3.735259 \\
\hline$y-75$ & $y^{-}$ & 75 & 9242.493 & 9242.525 & 0.032002 & 3.462489 \\
\hline$x+77$ & $x+$ & 77 & 9520.631 & 9520.68 & 0.04957 & 5.206585 \\
\hline y85 & y & 85 & 10429.04 & 10429.04 & -0.00093 & -0.08946 \\
\hline z87 & $z$ & 87 & 10597.14 & 10597.22 & 0.07384 & 6.967915 \\
\hline z91 & $z$ & 91 & 11059.37 & 11059.4 & 0.020813 & 1.881933 \\
\hline$x+95$ & $x+$ & 95 & 11492.53 & 11492.54 & 0.010817 & 0.941217 \\
\hline$x+100$ & $x+$ & 100 & 12171.85 & 12171.87 & 0.024952 & 2.049974 \\
\hline z101 & $z$ & 101 & 12243.87 & 12243.92 & 0.048789 & 3.984771 \\
\hline$x+117$ & $x+$ & 117 & 14079.7 & 14079.72 & 0.021924 & 1.557133 \\
\hline z118 & $z$ & 118 & 14149.78 & 14149.79 & 0.013299 & 0.939873 \\
\hline
\end{tabular}




\begin{tabular}{|c|c|c|c|c|c|c|}
\hline y119 & y & 119 & 14268.81 & 14268.83 & 0.018547 & 1.299828 \\
\hline z120 & z & 120 & 14353.84 & 14353.88 & 0.040902 & 2.849552 \\
\hline z122 & $z$ & 122 & 14539.9 & 14539.95 & 0.052291 & 3.596379 \\
\hline y123 & y & 123 & 14670.95 & 14670.93 & -0.01929 & -1.31505 \\
\hline z123 & $z$ & 123 & 14654.93 & 14654.99 & 0.064414 & 4.395381 \\
\hline$x+124$ & $x+$ & 124 & 14811.02 & 14811.01 & -0.0111 & -0.74924 \\
\hline z126 & $z$ & 126 & 14966.15 & 14966.19 & 0.042264 & 2.823973 \\
\hline$y-126$ & $y-$ & 126 & 14981.16 & 14981.22 & 0.061842 & 4.127987 \\
\hline$x+129$ & $x+$ & 129 & 15421.41 & 15421.42 & 0.005902 & 0.382713 \\
\hline z130 & $z$ & 130 & 15541.47 & 15541.49 & 0.025937 & 1.66889 \\
\hline x131 & $x$ & 131 & 15670.5 & 15670.56 & 0.058238 & 3.71641 \\
\hline y134 & y & 134 & 16001.67 & 16001.69 & 0.016245 & 1.015206 \\
\hline z134 & $z$ & 134 & 15985.65 & 15985.72 & 0.071135 & 4.449928 \\
\hline$x+135$ & $x+$ & 135 & 16129.71 & 16129.8 & 0.088511 & 5.487451 \\
\hline y136 & $y$ & 136 & 16199.77 & 16199.82 & 0.051753 & 3.194675 \\
\hline z136 & z & 136 & 16183.75 & 16183.85 & 0.092569 & 5.719872 \\
\hline x137 & $x$ & 137 & 16340.78 & 16340.83 & 0.051974 & 3.180632 \\
\hline y138 & y & 138 & 16477.86 & 16477.98 & 0.115369 & 7.001454 \\
\hline$x+139$ & $x+$ & 139 & 16633.89 & 16633.93 & 0.036881 & 2.217218 \\
\hline z139 & $z$ & 139 & 16590.89 & 16590.95 & 0.060294 & 3.634164 \\
\hline$y-140$ & $y^{-}$ & 140 & 16720.92 & 16721.01 & 0.084426 & 5.049125 \\
\hline z140 & $z$ & 140 & 16705.91 & 16706.01 & 0.099761 & 5.971598 \\
\hline y141 & y & 141 & 16821 & 16821.04 & 0.036511 & 2.170561 \\
\hline$x 143$ & $x$ & 143 & 17131.11 & 17131.04 & -0.06373 & -3.7199 \\
\hline y143 & y & 143 & 17105.13 & 17105.17 & 0.040539 & 2.369991 \\
\hline z144 & z & 144 & 17203.15 & 17203.13 & -0.02366 & -1.37562 \\
\hline y144 & y & 144 & 17219.17 & 17219.21 & 0.041554 & 2.413241 \\
\hline$y-145$ & $y-$ & 145 & 17346.22 & 17346.3 & 0.076249 & 4.395714 \\
\hline z147 & $z$ & 147 & 17557.38 & 17557.37 & -0.01277 & -0.72722 \\
\hline$x+147$ & $x+$ & 147 & 17600.38 & 17600.38 & -0.0052 & -0.29522 \\
\hline y147 & y & 147 & 17573.4 & 17573.49 & 0.094175 & 5.358952 \\
\hline z149 & $z$ & 149 & 17798.52 & 17798.56 & 0.035265 & 1.981344 \\
\hline y149 & y & 149 & 17814.54 & 17814.58 & 0.038229 & 2.145944 \\
\hline z150 & $z$ & 150 & 17899.57 & 17899.58 & 0.010779 & 0.602193 \\
\hline$x+150$ & $x+$ & 150 & 17942.57 & 17942.68 & 0.107219 & 5.975673 \\
\hline x151 & $x$ & 151 & 18054.65 & 18054.72 & 0.065163 & 3.609209 \\
\hline y152 & $y$ & 152 & 18099.71 & 18099.75 & 0.042475 & 2.346723 \\
\hline x152 & $x$ & 152 & 18125.69 & 18125.77 & 0.080788 & 4.4571 \\
\hline$x+153$ & $x+$ & 153 & 18213.73 & 18213.82 & 0.092935 & 5.102468 \\
\hline z154 & $z$ & 154 & 18298.82 & 18298.87 & 0.054855 & 2.997735 \\
\hline y156 & y & 156 & 18470.93 & 18470.98 & 0.051201 & 2.771978 \\
\hline x157 & $x$ & 157 & 18553.93 & 18553.94 & 0.014831 & 0.799346 \\
\hline$y-157$ & $y-$ & 157 & 18526.94 & 18527 & 0.056364 & 3.042274 \\
\hline
\end{tabular}




$\begin{array}{llrrrrr}x+158 & x+ & 158 & 18641.97 & 18642.04 & 0.069703 & 3.739035 \\ z 159 & z & 159 & 18670 & 18670.11 & 0.111934 & 5.995394 \\ y 160 & y & 160 & 18743.04 & 18743.05 & 0.011162 & 0.595528 \\ y-161 & y- & 161 & 18841.1 & 18841.14 & 0.039604 & 2.102002 \\ z 162 & z & 162 & 18925.16 & 18925.2 & 0.045207 & 2.388725 \\ y 164 & y & 164 & 19153.33 & 19153.38 & 0.055856 & 2.916256 \\ z 164 & z & 164 & 19137.31 & 19137.46 & 0.149132 & 7.792736 \\ z 165 & z & 165 & 19265.4 & 19265.4 & -0.00705 & -0.3662 \\ x 165 & x & 165 & 19307.4 & 19307.47 & 0.064694 & 3.350736 \\ z 169 & z & 169 & 19789.6 & 19789.76 & 0.164769 & 8.326041\end{array}$


Table S4D. Summary of UVPD mass spectrum for G12S•GDPnP complex: fragment ions incorporating mass shift of +542.9526 Da (GDPnp)

\begin{tabular}{|c|c|c|c|c|c|c|}
\hline & Ion & & Theoretical & Experimental & & $\mathrm{ppm}$ \\
\hline Ion & Type & $\underline{\text { Residue }}$ & $\underline{\text { Mass }}$ & $\underline{\text { Mass }}$ & $\underline{\Delta \text { mass }}$ & error \\
\hline c1 & c & 1 & 617.0004 & 617 & -0.00036 & -0.58347 \\
\hline b123 & b & 123 & 14398.73 & 14398.87 & 0.134355 & 9.331029 \\
\hline a124 & $a$ & 124 & 14526.84 & 14526.93 & 0.093346 & 6.425761 \\
\hline a134 & a & 134 & 15626.39 & 15626.51 & 0.118249 & 7.567263 \\
\hline b136 & $b$ & 136 & 15881.52 & 15881.59 & 0.067364 & 4.241659 \\
\hline$a+137$ & $a+$ & 137 & 15941.57 & 15941.61 & 0.03981 & 2.497243 \\
\hline c137 & c & 137 & 15985.58 & 15985.72 & 0.142445 & 8.910842 \\
\hline$a+139$ & $a+$ & 139 & 16161.65 & 16161.8 & 0.151367 & 9.36581 \\
\hline a141 & $a$ & 141 & 16370.78 & 16370.85 & 0.071444 & 4.364116 \\
\hline c143 & c & 143 & 16675.96 & 16676.01 & 0.055766 & 3.344096 \\
\hline$a+144$ & $a+$ & 144 & 16760.98 & 16760.97 & -0.01475 & -0.8799 \\
\hline a145 & $a$ & 145 & 16861.02 & 16861.02 & -0.00529 & -0.31356 \\
\hline a149 & $a$ & 149 & 17248.24 & 17248.23 & -0.0073 & -0.423 \\
\hline a151 & a & 151 & 17532.4 & 17532.36 & -0.03461 & -1.97429 \\
\hline b165 & b & 165 & 19195.23 & 19195.32 & 0.086864 & 4.52529 \\
\hline a165 & a & 165 & 19167.24 & 19167.39 & 0.156969 & 8.189443 \\
\hline a166 & $a$ & 166 & 19295.33 & 19295.44 & 0.105955 & 5.491224 \\
\hline a167 & a & 167 & 19432.39 & 19432.51 & 0.122972 & 6.328197 \\
\hline$y-6$ & $y-$ & 6 & 1366.443 & 1366.452 & 0.009244 & 6.765034 \\
\hline$x+9$ & $x+$ & 9 & 1792.666 & 1792.679 & 0.01362 & 7.597607 \\
\hline y20 & y & 20 & 2974.25 & 2974.226 & -0.02387 & -8.02454 \\
\hline$x 72$ & $x$ & 72 & 8814.249 & 8814.236 & -0.01261 & -1.4303 \\
\hline x77 & $x$ & 77 & 9520.615 & 9520.68 & 0.065195 & 6.847772 \\
\hline$x+78$ & $x+$ & 78 & 9636.65 & 9636.671 & 0.021712 & 2.253062 \\
\hline y80 & y & 80 & 9885.774 & 9885.852 & 0.078353 & 7.925834 \\
\hline$x+84$ & $x+$ & 84 & 10342.98 & 10343.04 & 0.059072 & 5.711311 \\
\hline$y-85$ & $y-$ & 85 & 10429.03 & 10429.04 & 0.014692 & 1.408764 \\
\hline z85 & $z$ & 85 & 10414.02 & 10414.05 & 0.030869 & 2.964178 \\
\hline y86 & y & 86 & 10543.12 & 10543.15 & 0.032307 & 3.064274 \\
\hline$x+86$ & $x+$ & 86 & 10570.11 & 10570.15 & 0.041555 & 3.931367 \\
\hline y87 & y & 87 & 10614.16 & 10614.17 & 0.01258 & 1.18521 \\
\hline y88 & y & 88 & 10761.22 & 10761.25 & 0.028934 & 2.688728 \\
\hline y89 & y & 89 & 10860.29 & 10860.32 & 0.027337 & 2.517151 \\
\hline y90 & y & 90 & 10963.3 & 10963.33 & 0.024602 & 2.244032 \\
\hline$x+90$ & $x+$ & 90 & 10990.29 & 10990.32 & 0.033264 & 3.026669 \\
\hline y91 & y & 91 & 11076.39 & 11076.4 & 0.015713 & 1.418604 \\
\hline$x+91$ & $x+$ & 91 & 11103.37 & 11103.39 & 0.018286 & 1.646884 \\
\hline y95 & y & 95 & 11466.54 & 11466.56 & 0.020438 & 1.782404 \\
\hline
\end{tabular}




\begin{tabular}{|c|c|c|c|c|c|c|}
\hline x95 & $x$ & 95 & 11492.52 & 11492.54 & 0.026442 & 2.300801 \\
\hline$z 96$ & $z$ & 96 & 11551.57 & 11551.58 & 0.01378 & 1.192912 \\
\hline$x 100$ & $x$ & 100 & 12171.83 & 12171.87 & 0.040577 & 3.333681 \\
\hline y101 & $y$ & 101 & 12260.88 & 12260.9 & 0.026621 & 2.171215 \\
\hline y110 & y & 110 & 13312.32 & 13312.31 & -0.00945 & -0.70957 \\
\hline z110 & $z$ & 110 & 13296.3 & 13296.31 & 0.013713 & 1.03134 \\
\hline y113 & $y$ & 113 & 13599.43 & 13599.32 & -0.10499 & -7.7204 \\
\hline z113 & $z$ & 113 & 13583.41 & 13583.43 & 0.017931 & 1.320066 \\
\hline$x 117$ & $x$ & 117 & 14079.69 & 14079.72 & 0.037549 & 2.666892 \\
\hline$y-119$ & $y-$ & 119 & 14268.79 & 14268.83 & 0.034172 & 2.394879 \\
\hline$x+119$ & $x+$ & 119 & 14296.79 & 14296.83 & 0.040966 & 2.865397 \\
\hline$x+121$ & $x+$ & 121 & 14526.88 & 14526.93 & 0.054456 & 3.748635 \\
\hline$y-123$ & $y-$ & 123 & 14670.93 & 14670.93 & -0.00367 & -0.25002 \\
\hline x124 & $x$ & 124 & 14811 & 14811.01 & 0.004528 & 0.305719 \\
\hline y124 & $y$ & 124 & 14785.02 & 14785.08 & 0.0585 & 3.956707 \\
\hline$x+125$ & $x+$ & 125 & 14911.08 & 14911.11 & 0.033273 & 2.231426 \\
\hline y125 & $y$ & 125 & 14884.09 & 14884.18 & 0.085549 & 5.74768 \\
\hline$x+126$ & $x+$ & 126 & 15010.15 & 15010.23 & 0.084737 & 5.645312 \\
\hline y127 & $y$ & 127 & 15111.22 & 15111.23 & 0.00812 & 0.537349 \\
\hline z127 & $z$ & 127 & 15095.2 & 15095.27 & 0.071074 & 4.708384 \\
\hline y128 & y & 128 & 15239.31 & 15239.38 & 0.063794 & 4.186146 \\
\hline$x+128$ & $x+$ & 128 & 15266.3 & 15266.38 & 0.078657 & 5.152327 \\
\hline x129 & $x$ & 129 & 15421.39 & 15421.42 & 0.021527 & 1.395918 \\
\hline z129 & z & 129 & 15379.4 & 15379.42 & 0.022953 & 1.492451 \\
\hline$x+130$ & $x+$ & 130 & 15585.47 & 15585.51 & 0.048739 & 3.127206 \\
\hline$x+131$ & $x+$ & 131 & 15672.5 & 15672.54 & 0.04304 & 2.74621 \\
\hline$x+132$ & $x+$ & 132 & 15787.52 & 15787.63 & 0.101725 & 6.443377 \\
\hline$x+133$ & $x+$ & 133 & 15916.57 & 15916.64 & 0.070226 & 4.41213 \\
\hline$x+134$ & $x+$ & 134 & 16029.65 & 16029.57 & -0.07804 & -4.86867 \\
\hline$y-134$ & $y-$ & 134 & 16001.66 & 16001.69 & 0.03187 & 1.991671 \\
\hline x135 & $x$ & 135 & 16129.69 & 16129.8 & 0.104136 & 6.456168 \\
\hline$y-136$ & $y^{-}$ & 136 & 16199.76 & 16199.82 & 0.067378 & 4.1592 \\
\hline z137 & $z$ & 137 & 16299.77 & 16299.69 & -0.08503 & -5.21694 \\
\hline y137 & $y$ & 137 & 16315.79 & 16315.91 & 0.113975 & 6.985564 \\
\hline$x+138$ & $x+$ & 138 & 16505.84 & 16505.91 & 0.072059 & 4.365665 \\
\hline$y-138$ & $y^{-}$ & 138 & 16477.85 & 16477.98 & 0.130994 & 7.949706 \\
\hline x139 & $x$ & 139 & 16633.88 & 16633.93 & 0.052506 & 3.15657 \\
\hline y139 & $y$ & 139 & 16607.9 & 16607.96 & 0.059095 & 3.558247 \\
\hline$x+140$ & $x+$ & 140 & 16749.91 & 16750.01 & 0.095066 & 5.67561 \\
\hline$x+141$ & $x+$ & 141 & 16848.98 & 16849.02 & 0.041596 & 2.468753 \\
\hline$y-141$ & $y^{-}$ & 141 & 16820.98 & 16821.04 & 0.052136 & 3.099464 \\
\hline z141 & $z$ & 141 & 16805.97 & 16806.04 & 0.067471 & 4.014703 \\
\hline y142 & y & 142 & 16969.06 & 16969.08 & 0.023265 & 1.371025 \\
\hline
\end{tabular}




\begin{tabular}{|c|c|c|c|c|c|c|}
\hline$y-143$ & $y^{-}$ & 143 & 17105.11 & 17105.17 & 0.056164 & 3.283465 \\
\hline$y-144$ & $y^{-}$ & 144 & 17219.16 & 17219.21 & 0.057179 & 3.320664 \\
\hline$x+145$ & $x+$ & 145 & 17375.21 & 17375.25 & 0.0447 & 2.572629 \\
\hline x147 & $x$ & 147 & 17600.37 & 17600.38 & 0.010429 & 0.592544 \\
\hline$y-147$ & $y-$ & 147 & 17573.38 & 17573.49 & 0.1098 & 6.248088 \\
\hline y148 & $y$ & 148 & 17702.45 & 17702.5 & 0.052649 & 2.974108 \\
\hline$x+148$ & $x+$ & 148 & 17729.44 & 17729.5 & 0.066339 & 3.741742 \\
\hline$y-149$ & $y-$ & 149 & 17814.52 & 17814.58 & 0.053854 & 3.023041 \\
\hline$x+149$ & $x+$ & 149 & 17842.52 & 17842.58 & 0.05723 & 3.207505 \\
\hline x150 & $x$ & 150 & 17942.56 & 17942.68 & 0.122844 & 6.846515 \\
\hline y151 & $y$ & 151 & 18029.66 & 18029.72 & 0.060041 & 3.330123 \\
\hline$y-152$ & $y-$ & 152 & 18099.69 & 18099.75 & 0.0581 & 3.210001 \\
\hline z153 & $z$ & 153 & 18171.71 & 18171.8 & 0.084373 & 4.643095 \\
\hline x153 & $x$ & 153 & 18213.71 & 18213.82 & 0.10856 & 5.960344 \\
\hline y154 & $y$ & 154 & 18315.83 & 18315.86 & 0.029486 & 1.609864 \\
\hline$x+155$ & $x+$ & 155 & 18399.84 & 18399.85 & 0.0141 & 0.766309 \\
\hline y155 & $y$ & 155 & 18372.85 & 18372.91 & 0.059051 & 3.214036 \\
\hline$y-156$ & $y-$ & 156 & 18470.91 & 18470.98 & 0.066826 & 3.617907 \\
\hline$x+156$ & $x+$ & 156 & 18498.9 & 18498.99 & 0.085582 & 4.626326 \\
\hline z157 & $z$ & 157 & 18512.92 & 18513 & 0.075104 & 4.056842 \\
\hline x158 & $x$ & 158 & 18641.95 & 18642.04 & 0.085328 & 4.577203 \\
\hline z158 & $z$ & 158 & 18599.95 & 18600.08 & 0.126082 & 6.778619 \\
\hline y159 & y & 159 & 18687.01 & 18687.03 & 0.018205 & 0.974206 \\
\hline$x+160$ & $x+$ & 160 & 18771.02 & 18771.04 & 0.019909 & 1.060623 \\
\hline$y-160$ & $y-$ & 160 & 18743.02 & 18743.05 & 0.026787 & 1.429174 \\
\hline z161 & $z$ & 161 & 18827.08 & 18827.12 & 0.039385 & 2.091934 \\
\hline$y-164$ & $y-$ & 164 & 19153.31 & 19153.38 & 0.071481 & 3.732046 \\
\hline$x+166$ & $x+$ & 166 & 19472.46 & 19472.63 & 0.162709 & 8.355849 \\
\hline y168 & $y$ & 168 & 19675.57 & 19675.75 & 0.178669 & 9.080754 \\
\hline$x+168$ & $x+$ & 168 & 19702.55 & 19702.75 & 0.193532 & 9.822684 \\
\hline
\end{tabular}


Table S5A. Summary of UVPD mass spectrum for G12V•GDP complex: apo fragment ions

Column "Ions" is the fragment ion identity $(a, b, c, x, y, z)$ along with the number of residues in the fragment ion. A plus or minus sign indicates the fragment ion has one additional hydrogen atom or one less hydrogen atom than a conventional fragment ion. Column "Ion Type" is the type of fragment ion $(a, b, c, x, y, z)$. A plus sign indicates a fragment ion $(a, x)$ with an additional hydrogen atom; a minus sign indicates a fragment ion ( $y$ ) that is missing one hydrogen atom (the addition of one hydrogen atom for $a / x$ ions is not uncommon in UVPD mass spectra of intact proteins); Column "Residue" is the number of residues in the fragment ion. Column "Theoretical Mass" is the theoretical mass of the fragment ion; Column "Experimental Mass" is the experimentally measured mass of the fragment ion. Column "Delta mass" is the mass difference between the theoretical and experimental masses; and column "ppm error" is the mass error in ppm. The "apo" table (Table S5A) provides the fragment ions that do not retain the ligand. Tables S5B, S5C, and S5D provide the fragment ions that incorporate the ligand based on the mass shift corresponding to the retained ligand (with or without an additional hydrogen atom).

\begin{tabular}{|c|c|c|c|c|c|c|}
\hline Ion & $\frac{\text { Ion }}{\text { Tyne }}$ & Recidue & Theoretical & Experimental & Amass & $\frac{\mathrm{ppm}}{\text { error }}$ \\
\hline$\overline{a+4}$ & $\overline{a+}$ & $\overline{4}$ & 391.1651 & 391.167 & $\overline{0.001879}$ & $\overline{4.803516}$ \\
\hline a4 & $\mathrm{a}$ & 4 & 390.1573 & 390.1592 & 0.001892 & 4.849326 \\
\hline b4 & $b$ & 4 & 418.1522 & 418.1542 & 0.001972 & 4.715986 \\
\hline c4 & c & 4 & 435.1785 & 435.1808 & 0.002253 & 5.177186 \\
\hline a5 & a & 5 & 553.2206 & 553.2233 & 0.002618 & 4.732289 \\
\hline b5 & $b$ & 5 & 581.2156 & 581.2183 & 0.002759 & 4.746948 \\
\hline$a+5$ & $a+$ & 5 & 554.2285 & 554.2314 & 0.002911 & 5.252289 \\
\hline c5 & c & 5 & 598.2419 & 598.245 & 0.003132 & 5.235341 \\
\hline$a+6$ & $a+$ & 6 & 682.3234 & 682.3267 & 0.003288 & 4.818782 \\
\hline b7 & $b$ & 7 & 822.3946 & 822.3985 & 0.003976 & 4.834662 \\
\hline$a+7$ & $a+$ & 7 & 795.4075 & 795.4116 & 0.004128 & 5.189752 \\
\hline a8 & $\mathrm{a}$ & 8 & 893.4681 & 893.4723 & 0.004212 & 4.714214 \\
\hline b8 & $b$ & 8 & 921.463 & 921.4674 & 0.004414 & 4.790209 \\
\hline c8 & c & 8 & 938.4893 & 938.4941 & 0.004786 & 5.099685 \\
\hline$a+8$ & $a+$ & 8 & 894.4759 & 894.4807 & 0.004809 & 5.376297 \\
\hline a9 & a & 9 & 992.5365 & 992.5411 & 0.004588 & 4.6225 \\
\hline b9 & $b$ & 9 & 1020.531 & 1020.536 & 0.00479 & 4.693633 \\
\hline$a+9$ & $a+$ & 9 & 993.5443 & 993.5491 & 0.00482 & 4.851286 \\
\hline c9 & c & 9 & 1037.558 & 1037.563 & 0.005346 & 5.152485 \\
\hline a10 & $a$ & 10 & 1091.605 & 1091.595 & -0.01035 & -9.48603 \\
\hline$a+10$ & $a+$ & 10 & 1092.613 & 1092.617 & 0.004281 & 3.918102 \\
\hline c10 & $\mathrm{c}$ & 10 & 1136.626 & 1136.631 & 0.004685 & 4.121848 \\
\hline b10 & $b$ & 10 & 1119.6 & 1119.606 & 0.00596 & 5.323331 \\
\hline$a+11$ & $a+$ & 11 & 1149.634 & 1149.639 & 0.004672 & 4.063874 \\
\hline b11 & $b$ & 11 & 1176.621 & 1176.627 & 0.005252 & 4.463628 \\
\hline c11 & c & 11 & 1193.648 & 1193.654 & 0.006662 & 5.581212 \\
\hline$a+12$ & $a+$ & 12 & 1220.671 & 1220.676 & 0.004793 & 3.926502 \\
\hline
\end{tabular}




\begin{tabular}{|c|c|c|c|c|c|c|}
\hline a12 & $a$ & 12 & 1219.663 & 1219.669 & 0.005172 & 4.240514 \\
\hline c12 & c & 12 & 1264.685 & 1264.69 & 0.005319 & 4.205791 \\
\hline b12 & $b$ & 12 & 1247.658 & 1247.664 & 0.005496 & 4.405052 \\
\hline c13 & c & 13 & 1363.753 & 1363.757 & 0.004292 & 3.147197 \\
\hline a13 & a & 13 & 1318.732 & 1318.737 & 0.004877 & 3.69825 \\
\hline$a+13$ & $a+$ & 13 & 1319.74 & 1319.745 & 0.004986 & 3.777993 \\
\hline b13 & $b$ & 13 & 1346.727 & 1346.732 & 0.005201 & 3.861956 \\
\hline a14 & $a$ & 14 & 1375.753 & 1375.758 & 0.004291 & 3.119018 \\
\hline$a+14$ & $a+$ & 14 & 1376.761 & 1376.766 & 0.005255 & 3.816906 \\
\hline c14 & c & 14 & 1420.775 & 1420.78 & 0.005903 & 4.154776 \\
\hline b14 & $b$ & 14 & 1403.748 & 1403.755 & 0.00669 & 4.765812 \\
\hline b15 & $b$ & 15 & 1502.817 & 1502.821 & 0.004443 & 2.956448 \\
\hline$a+15$ & $a+$ & 15 & 1475.83 & 1475.834 & 0.004838 & 3.278135 \\
\hline c15 & c & 15 & 1519.843 & 1519.849 & 0.006463 & 4.252413 \\
\hline a15 & $\mathrm{a}$ & 15 & 1474.822 & 1474.829 & 0.006804 & 4.613439 \\
\hline$a+16$ & $a+$ & 16 & 1532.851 & 1532.854 & 0.003276 & 2.137173 \\
\hline b16 & $b$ & 16 & 1559.838 & 1559.844 & 0.005688 & 3.646532 \\
\hline c16 & c & 16 & 1576.864 & 1576.87 & 0.005877 & 3.727017 \\
\hline c17 & c & 17 & 1704.959 & 1704.965 & 0.005888 & 3.453455 \\
\hline$a+17$ & $a+$ & 17 & 1660.946 & 1660.953 & 0.006582 & 3.962783 \\
\hline a17 & $\mathrm{a}$ & 17 & 1659.938 & 1659.945 & 0.006595 & 3.97304 \\
\hline b17 & $b$ & 17 & 1687.933 & 1687.943 & 0.009605 & 5.690392 \\
\hline c18 & c & 18 & 1791.991 & 1791.996 & 0.004863 & 2.713741 \\
\hline$a+18$ & $a+$ & 18 & 1747.978 & 1747.983 & 0.00507 & 2.900476 \\
\hline a18 & a & 18 & 1746.97 & 1746.978 & 0.007815 & 4.473459 \\
\hline$a+19$ & $a+$ & 19 & 1819.015 & 1819.021 & 0.006046 & 3.323759 \\
\hline a19 & $\mathrm{a}$ & 19 & 1818.007 & 1818.015 & 0.008059 & 4.432876 \\
\hline$a+20$ & $a+$ & 20 & 1932.099 & 1932.106 & 0.006783 & 3.510673 \\
\hline c20 & c & 20 & 1976.113 & 1976.121 & 0.008178 & 4.138428 \\
\hline a20 & a & 20 & 1931.091 & 1931.1 & 0.00896 & 4.639863 \\
\hline b20 & b & 20 & 1959.086 & 1959.098 & 0.012214 & 6.234539 \\
\hline b21 & $b$ & 21 & 2060.134 & 2060.121 & -0.01325 & -6.43114 \\
\hline a21 & $a$ & 21 & 2032.139 & 2032.127 & -0.01174 & -5.77864 \\
\hline$a+21$ & $a+$ & 21 & 2033.147 & 2033.156 & 0.009363 & 4.605161 \\
\hline$a+22$ & $a+$ & 22 & 2146.231 & 2146.236 & 0.004967 & 2.314275 \\
\hline c22 & c & 22 & 2190.244 & 2190.253 & 0.008911 & 4.068496 \\
\hline a22 & $a$ & 22 & 2145.223 & 2145.232 & 0.009178 & 4.278343 \\
\hline$a+23$ & $a+$ & 23 & 2274.29 & 2274.294 & 0.004981 & 2.19012 \\
\hline c23 & c & 23 & 2318.303 & 2318.312 & 0.009217 & 3.975753 \\
\hline$a+24$ & $a+$ & 24 & 2387.374 & 2387.381 & 0.00715 & 2.99491 \\
\hline c24 & c & 24 & 2431.387 & 2431.397 & 0.00963 & 3.960702 \\
\hline b24 & $b$ & 24 & 2414.361 & 2414.37 & 0.009806 & 4.061531 \\
\hline$a+25$ & $a+$ & 25 & 2500.458 & 2500.464 & 0.006831 & 2.731887 \\
\hline
\end{tabular}




\begin{tabular}{|c|c|c|c|c|c|c|}
\hline a25 & a & 25 & 2499.45 & 2499.46 & 0.010261 & 4.105304 \\
\hline b25 & $b$ & 25 & 2527.445 & 2527.455 & 0.010463 & 4.139754 \\
\hline$c 25$ & c & 25 & 2544.471 & 2544.482 & 0.01053 & 4.138385 \\
\hline$c 26$ & c & 26 & 2672.53 & 2672.539 & 0.009079 & 3.397156 \\
\hline a26 & $a$ & 26 & 2627.508 & 2627.518 & 0.010031 & 3.817685 \\
\hline$a+27$ & $a+$ & 27 & 2742.559 & 2742.567 & 0.007616 & 2.776957 \\
\hline$c 27$ & c & 27 & 2786.573 & 2786.583 & 0.010583 & 3.797856 \\
\hline a27 & a & 27 & 2741.551 & 2741.562 & 0.010802 & 3.940105 \\
\hline$a+28$ & $a+$ & 28 & 2879.618 & 2879.627 & 0.009252 & 3.212915 \\
\hline c28 & c & 28 & 2923.631 & 2923.642 & 0.010267 & 3.511729 \\
\hline a28 & a & 28 & 2878.61 & 2878.622 & 0.011876 & 4.125602 \\
\hline b28 & $b$ & 28 & 2906.605 & 2906.621 & 0.015815 & 5.441056 \\
\hline a29 & $a$ & 29 & 3025.679 & 3025.688 & 0.009384 & 3.101453 \\
\hline$a+29$ & $a+$ & 29 & 3026.686 & 3026.696 & 0.009446 & 3.120894 \\
\hline c29 & $c$ & 29 & 3070.7 & 3070.712 & 0.011681 & 3.804019 \\
\hline c30 & c & 30 & 3169.768 & 3169.779 & 0.011142 & 3.515083 \\
\hline a30 & a & 30 & 3124.747 & 3124.759 & 0.012165 & 3.893115 \\
\hline$a+31$ & $a+$ & 31 & 3240.782 & 3240.761 & -0.02125 & -6.5583 \\
\hline c31 & c & 31 & 3284.795 & 3284.777 & -0.01804 & -5.49288 \\
\hline b31 & $b$ & 31 & 3267.769 & 3267.785 & 0.016117 & 4.932111 \\
\hline$a+32$ & $a+$ & 32 & 3369.824 & 3369.835 & 0.010422 & 3.092733 \\
\hline c33 & $c$ & 33 & 3576.901 & 3576.913 & 0.011583 & 3.238278 \\
\hline a33 & a & 33 & 3531.88 & 3531.897 & 0.016629 & 4.708257 \\
\hline b33 & $b$ & 33 & 3559.875 & 3559.904 & 0.029356 & 8.246357 \\
\hline a34 & a & 34 & 3646.907 & 3646.897 & -0.00935 & -2.56464 \\
\hline$a+34$ & $a+$ & 34 & 3647.915 & 3647.927 & 0.012287 & 3.368217 \\
\hline c35 & c & 35 & 3788.981 & 3788.946 & -0.03523 & -9.29881 \\
\hline$a+35$ & $a+$ & 35 & 3744.967 & 3744.978 & 0.010458 & 2.792539 \\
\hline c36 & $c$ & 36 & 3890.028 & 3889.991 & -0.03763 & -9.67217 \\
\hline a36 & a & 36 & 3845.007 & 3845.02 & 0.012596 & 3.275937 \\
\hline a37 & a & 37 & 3958.091 & 3958.108 & 0.017159 & 4.33517 \\
\hline a38 & a & 38 & 4087.134 & 4087.147 & 0.012899 & 3.156001 \\
\hline c38 & c & 38 & 4132.155 & 4132.173 & 0.017807 & 4.309374 \\
\hline a39 & a & 39 & 4202.161 & 4202.172 & 0.011593 & 2.758819 \\
\hline b39 & $b$ & 39 & 4230.156 & 4230.189 & 0.032792 & 7.75196 \\
\hline$a+40$ & $a+$ & 40 & 4290.201 & 4290.164 & -0.03694 & -8.60986 \\
\hline c40 & $c$ & 40 & 4334.214 & 4334.179 & -0.03465 & -7.99361 \\
\hline a40 & a & 40 & 4289.193 & 4289.211 & 0.018138 & 4.228768 \\
\hline a41 & a & 41 & 4452.256 & 4452.269 & 0.012938 & 2.905942 \\
\hline c42 & c & 42 & 4653.379 & 4653.395 & 0.016002 & 3.438792 \\
\hline a42 & a & 42 & 4608.357 & 4608.374 & 0.017075 & 3.705225 \\
\hline c43 & c & 43 & 4781.474 & 4781.436 & -0.03733 & -7.80764 \\
\hline$a+43$ & $a+$ & 43 & 4737.46 & 4737.475 & 0.015365 & 3.243292 \\
\hline
\end{tabular}




\begin{tabular}{|c|c|c|c|c|c|c|}
\hline c44 & c & 44 & 4909.532 & 4909.494 & -0.03781 & -7.70073 \\
\hline a44 & a & 44 & 4864.511 & 4864.527 & 0.016001 & 3.289334 \\
\hline a45 & $a$ & 45 & 4963.579 & 4963.598 & 0.018636 & 3.754549 \\
\hline a46 & a & 46 & 5062.648 & 5062.667 & 0.01944 & 3.839888 \\
\hline a47 & a & 47 & 5175.732 & 5175.753 & 0.020951 & 4.04793 \\
\hline b47 & b & 47 & 5203.727 & 5203.754 & 0.027623 & 5.308311 \\
\hline a48 & $a$ & 48 & 5290.759 & 5290.776 & 0.016809 & 3.177049 \\
\hline$a+49$ & $a+$ & 49 & 5348.788 & 5348.802 & 0.014043 & 2.625449 \\
\hline c50 & c & 50 & 5521.844 & 5521.818 & -0.02591 & -4.69173 \\
\hline$a+50$ & $a+$ & 50 & 5477.831 & 5477.843 & 0.012834 & 2.342892 \\
\hline a50 & $\mathrm{a}$ & 50 & 5476.823 & 5476.844 & 0.021055 & 3.844382 \\
\hline c51 & c & 51 & 5622.892 & 5622.864 & -0.02788 & -4.95901 \\
\hline$a+51$ & $a+$ & 51 & 5578.878 & 5578.895 & 0.016302 & 2.922087 \\
\hline a53 & $a$ & 53 & 5793.964 & 5793.974 & 0.010589 & 1.827592 \\
\hline a54 & $a$ & 54 & 5907.048 & 5907.045 & -0.00255 & -0.43135 \\
\hline a55 & a & 55 & 6022.075 & 6022.075 & 0.000297 & 0.049319 \\
\hline a56 & a & 56 & 6135.159 & 6135.182 & 0.023635 & 3.852386 \\
\hline a57 & a & 57 & 6248.243 & 6248.253 & 0.010277 & 1.644782 \\
\hline$c 58$ & c & 58 & 6408.291 & 6408.26 & -0.03051 & -4.7604 \\
\hline$a+58$ & $a+$ & 58 & 6364.278 & 6364.302 & 0.024829 & 3.901302 \\
\hline c59 & $c$ & 59 & 6509.339 & 6509.31 & -0.02887 & -4.43501 \\
\hline a59 & a & 59 & 6464.317 & 6464.336 & 0.018421 & 2.849643 \\
\hline$a+60$ & $a+$ & 60 & 6536.362 & 6536.372 & 0.009375 & 1.434279 \\
\hline$a+61$ & $a+$ & 61 & 6593.384 & 6593.395 & 0.011352 & 1.721721 \\
\hline$a+62$ & $a+$ & 62 & 6721.442 & 6721.461 & 0.018934 & 2.81695 \\
\hline a63 & $a$ & 63 & 6849.477 & 6849.498 & 0.021118 & 3.083155 \\
\hline$a+63$ & $a+$ & 63 & 6850.485 & 6850.511 & 0.026281 & 3.836366 \\
\hline a64 & a & 64 & 6978.52 & 6978.546 & 0.025829 & 3.701215 \\
\hline$a+66$ & $a+$ & 66 & 7229.623 & 7229.561 & -0.06171 & -8.53503 \\
\hline a69 & a & 69 & 7586.794 & 7586.813 & 0.019364 & 2.55233 \\
\hline a70 & $a$ & 70 & 7701.821 & 7701.836 & 0.015373 & 1.996022 \\
\hline$a+71$ & $a+$ & 71 & 7830.887 & 7830.904 & 0.016351 & 2.08801 \\
\hline c72 & c & 72 & 8037.964 & 8037.945 & -0.01886 & -2.34699 \\
\hline a72 & a & 72 & 7992.943 & 7992.963 & 0.019928 & 2.493199 \\
\hline a73 & $a$ & 73 & 8123.983 & 8123.994 & 0.011176 & 1.37568 \\
\hline$a+74$ & $a+$ & 74 & 8281.092 & 8281.1 & 0.00771 & 0.931033 \\
\hline a75 & $a$ & 75 & 8381.132 & 8381.168 & 0.036506 & 4.355736 \\
\hline a77 & a & 77 & 8567.196 & 8567.225 & 0.028974 & 3.38197 \\
\hline a79 & a & 79 & 8771.286 & 8771.327 & 0.041277 & 4.705923 \\
\hline a80 & a & 80 & 8884.37 & 8884.383 & 0.013248 & 1.491158 \\
\hline a82 & a & 82 & 9086.448 & 9086.488 & 0.04075 & 4.484701 \\
\hline a83 & $a$ & 83 & 9233.516 & 9233.54 & 0.023732 & 2.570202 \\
\hline$a+84$ & $a+$ & 84 & 9305.561 & 9305.578 & 0.016761 & 1.801178 \\
\hline
\end{tabular}




\begin{tabular}{|c|c|c|c|c|c|c|}
\hline a85 & $a$ & 85 & 9417.637 & 9417.665 & 0.027928 & 2.9655 \\
\hline c86 & c & 86 & 9576.701 & 9576.69 & -0.01095 & -1.1433 \\
\hline a86 & $a$ & 86 & 9531.68 & 9531.718 & 0.037732 & 3.958589 \\
\hline c87 & c & 87 & 9690.744 & 9690.747 & 0.003128 & 0.322782 \\
\hline a87 & a & 87 & 9645.723 & 9645.756 & 0.033499 & 3.472938 \\
\hline a88 & $a$ & 88 & 9746.771 & 9746.789 & 0.017923 & 1.838865 \\
\hline$a+89$ & $a+$ & 89 & 9875.873 & 9875.884 & 0.010597 & 1.073016 \\
\hline$a+90$ & $a+$ & 90 & 9962.905 & 9962.912 & 0.006765 & 0.679016 \\
\hline a91 & $\mathrm{a}$ & 91 & 10108.97 & 10108.97 & 0.008192 & 0.81037 \\
\hline a92 & $a$ & 92 & 10238.01 & 10238 & -0.00425 & -0.41483 \\
\hline a93 & $\mathrm{a}$ & 93 & 10353.04 & 10353.05 & 0.016421 & 1.586105 \\
\hline$a+94$ & $a+$ & 94 & 10467.13 & 10467.16 & 0.031721 & 3.030532 \\
\hline y3 & $y$ & 3 & 403.2431 & 403.245 & 0.001876 & 4.652281 \\
\hline$x 3$ & $x$ & 3 & 429.2223 & 429.2243 & 0.001981 & 4.615324 \\
\hline y4 & y & 4 & 540.302 & 540.3046 & 0.002567 & 4.751047 \\
\hline$x 4$ & $x$ & 4 & 566.2813 & 566.2839 & 0.002672 & 4.718503 \\
\hline z4 & $z$ & 4 & 524.2832 & 524.2861 & 0.002838 & 5.413105 \\
\hline y5 & y & 5 & 668.3969 & 668.4002 & 0.003249 & 4.860884 \\
\hline$x+6$ & $x+$ & 6 & 851.4851 & 851.4845 & -0.0006 & -0.70469 \\
\hline y6 & y & 6 & 824.4981 & 824.502 & 0.003946 & 4.785942 \\
\hline$x 7$ & $x$ & 7 & 963.5614 & 963.5658 & 0.004402 & 4.568469 \\
\hline y7 & y & 7 & 937.5821 & 937.5865 & 0.004419 & 4.713187 \\
\hline z7 & $z$ & 7 & 921.5634 & 921.5682 & 0.004874 & 5.288839 \\
\hline y8 & y & 8 & 1066.625 & 1066.63 & 0.005225 & 4.89863 \\
\hline z8 & $z$ & 8 & 1050.606 & 1050.612 & 0.005924 & 5.638651 \\
\hline$y-9$ & $y-$ & 9 & 1221.718 & 1221.721 & 0.003493 & 2.859115 \\
\hline y9 & $y$ & 9 & 1222.726 & 1222.731 & 0.004945 & 4.044243 \\
\hline x9 & $x$ & 9 & 1248.705 & 1248.71 & 0.005295 & 4.240393 \\
\hline y10 & $y$ & 10 & 1321.794 & 1321.8 & 0.005871 & 4.44169 \\
\hline x10 & $x$ & 10 & 1347.773 & 1347.78 & 0.006099 & 4.525241 \\
\hline$y-11$ & $y-$ & 11 & 1433.87 & 1433.873 & 0.002268 & 1.581755 \\
\hline x11 & $x$ & 11 & 1460.858 & 1460.863 & 0.005657 & 3.872383 \\
\hline y11 & $y$ & 11 & 1434.878 & 1434.885 & 0.006528 & 4.549515 \\
\hline$x+12$ & $x+$ & 12 & 1562.913 & 1562.916 & 0.003422 & 2.189481 \\
\hline y12 & $y$ & 12 & 1535.926 & 1535.932 & 0.005601 & 3.64666 \\
\hline z13 & $z$ & 13 & 1682.971 & 1682.977 & 0.006128 & 3.641181 \\
\hline y13 & y & 13 & 1698.989 & 1698.997 & 0.007701 & 4.532695 \\
\hline$x 13$ & $x$ & 13 & 1724.969 & 1724.977 & 0.008586 & 4.977482 \\
\hline$x+14$ & $x+$ & 14 & 1873.045 & 1873.049 & 0.004128 & 2.203881 \\
\hline z14 & $z$ & 14 & 1830.039 & 1830.045 & 0.006321 & 3.454025 \\
\hline$x 14$ & $x$ & 14 & 1872.037 & 1872.046 & 0.009024 & 4.820418 \\
\hline y14 & y & 14 & 1846.058 & 1846.067 & 0.009651 & 5.227897 \\
\hline$x+15$ & $x+$ & 15 & 1944.082 & 1944.087 & 0.005226 & 2.688142 \\
\hline
\end{tabular}




\begin{tabular}{|c|c|c|c|c|c|c|}
\hline z15 & z & 15 & 1901.076 & 1901.082 & 0.005954 & 3.13191 \\
\hline y15 & y & 15 & 1917.095 & 1917.104 & 0.009528 & 4.97002 \\
\hline y16 & $y$ & 16 & 2032.122 & 2032.127 & 0.005537 & 2.724738 \\
\hline$x+17$ & $x+$ & 17 & 2174.136 & 2174.141 & 0.005668 & 2.606998 \\
\hline y17 & $y$ & 17 & 2147.149 & 2147.159 & 0.010092 & 4.700187 \\
\hline y19 & y & 19 & 2303.239 & 2303.247 & 0.0086 & 3.733873 \\
\hline$y-20$ & $y-$ & 20 & 2430.289 & 2430.297 & 0.00765 & 3.147787 \\
\hline y22 & $y$ & 22 & 2688.446 & 2688.458 & 0.012412 & 4.616794 \\
\hline y23 & $y$ & 23 & 2816.541 & 2816.552 & 0.011202 & 3.977219 \\
\hline y24 & y & 24 & 2887.578 & 2887.589 & 0.011201 & 3.879029 \\
\hline$x 25$ & $x$ & 25 & 3000.589 & 3000.598 & 0.009184 & 3.060732 \\
\hline y25 & y & 25 & 2974.61 & 2974.619 & 0.009397 & 3.15907 \\
\hline y26 & $y$ & 26 & 3075.658 & 3075.662 & 0.004489 & 1.459525 \\
\hline$x+26$ & $x+$ & 26 & 3102.645 & 3102.656 & 0.011296 & 3.640754 \\
\hline$x+27$ & $x+$ & 27 & 3231.687 & 3231.696 & 0.009095 & 2.814309 \\
\hline y27 & $y$ & 27 & 3204.7 & 3204.719 & 0.01854 & 5.785252 \\
\hline$z 27$ & $z$ & 27 & 3188.682 & 3188.701 & 0.019775 & 6.201623 \\
\hline$y-28$ & $y-$ & 28 & 3316.777 & 3316.785 & 0.008711 & 2.626355 \\
\hline y28 & $y$ & 28 & 3317.784 & 3317.796 & 0.011432 & 3.445673 \\
\hline$x+28$ & $x+$ & 28 & 3344.771 & 3344.785 & 0.013555 & 4.052584 \\
\hline$x+29$ & $x+$ & 29 & 3491.84 & 3491.849 & 0.009457 & 2.708305 \\
\hline z29 & $z$ & 29 & 3448.834 & 3448.845 & 0.01147 & 3.325762 \\
\hline$x 29$ & $x$ & 29 & 3490.832 & 3490.847 & 0.014905 & 4.269756 \\
\hline$y-30$ & $y-$ & 30 & 3560.898 & 3560.909 & 0.01088 & 3.055418 \\
\hline$x+30$ & $x+$ & 30 & 3588.893 & 3588.91 & 0.017871 & 4.979521 \\
\hline$y-31$ & $y-$ & 31 & 3673.982 & 3673.992 & 0.01056 & 2.874274 \\
\hline y31 & $y$ & 31 & 3674.99 & 3675.001 & 0.010914 & 2.969804 \\
\hline$x+31$ & $x+$ & 31 & 3701.977 & 3701.99 & 0.013675 & 3.693964 \\
\hline x31 & $x$ & 31 & 3700.969 & 3700.987 & 0.018052 & 4.877642 \\
\hline$x+32$ & $x+$ & 32 & 3758.998 & 3759.009 & 0.010473 & 2.786106 \\
\hline$y-32$ & $y-$ & 32 & 3731.003 & 3731.024 & 0.020303 & 5.441709 \\
\hline y33 & $y$ & 33 & 3895.074 & 3895.084 & 0.009742 & 2.501108 \\
\hline$x+33$ & $x+$ & 33 & 3922.061 & 3922.074 & 0.012032 & 3.067766 \\
\hline$y-34$ & $y-$ & 34 & 3981.099 & 3981.092 & -0.00653 & -1.63974 \\
\hline x34 & $x$ & 34 & 4008.086 & 4008.094 & 0.008213 & 2.049108 \\
\hline$x+35$ & $x+$ & 35 & 4165.195 & 4165.206 & 0.01146 & 2.751364 \\
\hline y35 & $y$ & 35 & 4138.208 & 4138.219 & 0.011734 & 2.835527 \\
\hline$y-35$ & $y-$ & 35 & 4137.2 & 4137.215 & 0.015775 & 3.812973 \\
\hline z35 & $z$ & 35 & 4122.189 & 4122.205 & 0.016461 & 3.993267 \\
\hline$y-36$ & $y^{-}$ & 36 & 4208.237 & 4208.246 & 0.008938 & 2.123938 \\
\hline z37 & $z$ & 37 & 4306.31 & 4306.322 & 0.012247 & 2.843966 \\
\hline$x+38$ & $x+$ & 38 & 4464.343 & 4464.357 & 0.014107 & 3.15992 \\
\hline y38 & $y$ & 38 & 4437.356 & 4437.372 & 0.016212 & 3.653527 \\
\hline
\end{tabular}




\begin{tabular}{|c|c|c|c|c|c|c|}
\hline y39 & y & 39 & 4565.414 & 4565.371 & -0.04348 & -9.52466 \\
\hline$y-39$ & $y^{-}$ & 39 & 4564.406 & 4564.369 & -0.03777 & -8.27577 \\
\hline z39 & $z$ & 39 & 4549.395 & 4549.413 & 0.017535 & 3.854358 \\
\hline y40 & $y$ & 40 & 4636.451 & 4636.461 & 0.009267 & 1.998727 \\
\hline$x+40$ & $x+$ & 40 & 4663.438 & 4663.452 & 0.013388 & 2.870836 \\
\hline$x+41$ & $x+$ & 41 & 4791.497 & 4791.523 & 0.026097 & 5.446517 \\
\hline$x+42$ & $x+$ & 42 & 4919.592 & 4919.627 & 0.035019 & 7.118267 \\
\hline$y-43$ & $y-$ & 43 & 4992.645 & 4992.666 & 0.02156 & 4.318359 \\
\hline$x+43$ & $x+$ & 43 & 5020.64 & 5020.666 & 0.026401 & 5.258487 \\
\hline$x+44$ & $x+$ & 44 & 5135.667 & 5135.679 & 0.012035 & 2.343409 \\
\hline$y-45$ & $y-$ & 45 & 5206.74 & 5206.751 & 0.011097 & 2.131282 \\
\hline$x 45$ & $x$ & 45 & 5233.727 & 5233.752 & 0.025302 & 4.834413 \\
\hline$x+47$ & $x+$ & 47 & 5491.884 & 5491.907 & 0.02342 & 4.264469 \\
\hline$x+48$ & $x+$ & 48 & 5578.916 & 5578.895 & -0.02125 & -3.80953 \\
\hline y50 & $y$ & 50 & 5762.066 & 5762.086 & 0.020105 & 3.4892 \\
\hline$x+50$ & $x+$ & 50 & 5789.053 & 5789.078 & 0.025682 & 4.436299 \\
\hline$y-50$ & $y-$ & 50 & 5761.058 & 5761.096 & 0.038577 & 6.696172 \\
\hline$y-52$ & $y-$ & 52 & 5979.094 & 5979.117 & 0.022991 & 3.845237 \\
\hline$x+53$ & $x+$ & 53 & 6135.184 & 6135.182 & -0.00135 & -0.21923 \\
\hline$y-53$ & $y-$ & 53 & 6107.189 & 6107.2 & 0.011423 & 1.870424 \\
\hline$y-54$ & $y-$ & 54 & 6221.232 & 6221.261 & 0.02974 & 4.780409 \\
\hline x54 & $x$ & 54 & 6248.219 & 6248.253 & 0.034252 & 5.481882 \\
\hline z56 & $z$ & 56 & 6362.311 & 6362.257 & -0.05363 & -8.42996 \\
\hline y59 & $y$ & 59 & 6721.522 & 6721.461 & -0.06106 & -9.0844 \\
\hline y61 & y & 61 & 6917.644 & 6917.658 & 0.01435 & 2.074406 \\
\hline$y-168$ & $y-$ & 168 & 19143.64 & 19143.48 & -0.16682 & -8.71396 \\
\hline
\end{tabular}


Table S5B. Summary of UVPD mass spectrum for G12V•GDP complex: fragment ions incorporating mass shift of +464.9937 Da (GDP)

\begin{tabular}{|c|c|c|c|c|c|c|}
\hline & $\underline{\text { Ion }}$ & & Theoretical & Experimental & & $\mathrm{ppm}$ \\
\hline Ion & Type & Residue & $\underline{\text { Mass }}$ & Mass & $\Delta$ mass & error \\
\hline b19 & $\mathrm{b}$ & 19 & 2310.996 & 2311 & 0.00409 & 1.7698 \\
\hline a25 & a & 25 & 2964.443 & 2964.429 & -0.014 & -4.72399 \\
\hline$a+26$ & $a+$ & 26 & 3093.51 & 3093.506 & -0.00375 & -1.2119 \\
\hline 28 & $b$ & 28 & 3371.599 & 3371.612 & 0.01279 & 3.793452 \\
\hline b49 & $b$ & 49 & 5840.769 & 5840.805 & 0.036501 & 6.249349 \\
\hline a111 & a & 111 & 13048.19 & 13048.24 & 0.052443 & 4.019178 \\
\hline b111 & $b$ & 111 & 13076.18 & 13076.25 & 0.064364 & 4.922231 \\
\hline$a+112$ & $a+$ & 112 & 13180.24 & 13180.32 & 0.0803 & 6.092452 \\
\hline$a+114$ & $a+$ & 114 & 13392.39 & 13392.37 & -0.02159 & -1.61181 \\
\hline c116 & c & 116 & 13592.49 & 13592.42 & -0.07387 & -5.43447 \\
\hline a121 & $\mathrm{a}$ & 121 & 14120.73 & 14120.7 & -0.02727 & -1.93149 \\
\hline a124 & a & 124 & 14460.92 & 14460.97 & 0.055173 & 3.815318 \\
\hline a126 & a & 126 & 14661.03 & 14660.98 & -0.05606 & -3.82354 \\
\hline a127 & $\mathrm{a}$ & 127 & 14776.06 & 14776.04 & -0.02194 & -1.48477 \\
\hline c127 & c & 127 & 14821.08 & 14821.09 & 0.007006 & 0.472705 \\
\hline a129 & a & 129 & 15005.2 & 15005.22 & 0.012667 & 0.844174 \\
\hline b132 & $b$ & 132 & 15360.35 & 15360.42 & 0.064898 & 4.225033 \\
\hline b133 & $b$ & 133 & 15475.38 & 15475.48 & 0.102655 & 6.63344 \\
\hline$a+134$ & $a+$ & 134 & 15561.48 & 15561.48 & 0.00263 & 0.169005 \\
\hline c135 & C & 135 & 15676.53 & 15676.52 & -0.00453 & -0.28929 \\
\hline a135 & a & 135 & 15631.5 & 15631.52 & 0.016439 & 1.051658 \\
\hline$a+140$ & $a+$ & 140 & 16208.81 & 16208.79 & -0.02673 & -1.64892 \\
\hline b140 & $b$ & 140 & 16235.8 & 16235.83 & 0.029837 & 1.837729 \\
\hline a142 & $a$ & 142 & 16451.93 & 16451.87 & -0.05382 & -3.27153 \\
\hline c143 & c & 143 & 16610.03 & 16609.98 & -0.05394 & -3.2475 \\
\hline c146 & c & 146 & 16927.16 & 16927.06 & -0.0917 & -5.41733 \\
\hline$a+150$ & $a+$ & 150 & 17339.42 & 17339.3 & -0.12145 & -7.00398 \\
\hline c151 & $c$ & 151 & 17511.49 & 17511.37 & -0.12293 & -7.02025 \\
\hline$a+156$ & $a+$ & 156 & 17924.66 & 17924.68 & 0.02051 & 1.144232 \\
\hline c159 & $c$ & 159 & 18379.86 & 18379.9 & 0.048033 & 2.61335 \\
\hline b161 & $b$ & 161 & 18574.98 & 18574.98 & 0.002556 & 0.137604 \\
\hline$a+161$ & $a+$ & 161 & 18547.99 & 18548.01 & 0.018613 & 1.003503 \\
\hline c165 & c & 165 & 19146.34 & 19146.43 & 0.095872 & 5.007329 \\
\hline a166 & a & 166 & 19229.41 & 19229.41 & -0.003 & -0.15606 \\
\hline$y-19$ & $y-$ & 19 & 2767.224 & 2767.216 & -0.00833 & -3.0095 \\
\hline y45 & $y$ & 45 & 5672.742 & 5672.73 & -0.01201 & -2.11679 \\
\hline z52 & z & 52 & 6429.077 & 6429.095 & 0.01871 & 2.910216 \\
\hline
\end{tabular}




\begin{tabular}{|c|c|c|c|c|c|c|}
\hline y54 & y & 54 & 6687.233 & 6687.173 & -0.06031 & -9.01838 \\
\hline$y-59$ & $y-$ & 59 & 7185.508 & 7185.57 & 0.061266 & 8.526332 \\
\hline$x+65$ & $x+$ & 65 & 7855.753 & 7855.783 & 0.030512 & 3.884028 \\
\hline z69 & $z$ & 69 & 8324.106 & 8324.092 & -0.01439 & -1.72931 \\
\hline$x+71$ & $x+$ & 71 & 8608.255 & 8608.19 & -0.06499 & -7.54973 \\
\hline$y-71$ & $y-$ & 71 & 8580.26 & 8580.235 & -0.02481 & -2.89187 \\
\hline$x+78$ & $x+$ & 78 & 9558.691 & 9558.703 & 0.011865 & 1.241275 \\
\hline y78 & y & 78 & 9531.704 & 9531.718 & 0.014082 & 1.477385 \\
\hline y84 & y & 84 & 10238.03 & 10238 & -0.0279 & -2.72484 \\
\hline$x 85$ & $x$ & 85 & 10378.05 & 10378.03 & -0.02292 & -2.20899 \\
\hline$x 86$ & $x$ & 86 & 10491.14 & 10491.14 & -0.00092 & -0.08807 \\
\hline y87 & $y$ & 87 & 10536.2 & 10536.17 & -0.03071 & -2.91519 \\
\hline$x+89$ & $x+$ & 89 & 10809.32 & 10809.29 & -0.02953 & -2.7319 \\
\hline x90 & $x$ & 90 & 10911.32 & 10911.33 & 0.00734 & 0.672696 \\
\hline x91 & $x$ & 91 & 11024.41 & 11024.38 & -0.0296 & -2.68504 \\
\hline$x+94$ & $x+$ & 94 & 11358.55 & 11358.52 & -0.03038 & -2.67446 \\
\hline$x+95$ & $x+$ & 95 & 11415.57 & 11415.54 & -0.02517 & -2.20471 \\
\hline$y-108$ & $y-$ & 108 & 13048.27 & 13048.24 & -0.02755 & -2.11154 \\
\hline$x+108$ & $x+$ & 108 & 13076.26 & 13076.25 & -0.01563 & -1.19537 \\
\hline$x+109$ & $x+$ & 109 & 13204.32 & 13204.34 & 0.016609 & 1.257843 \\
\hline x110 & $x$ & 110 & 13260.34 & 13260.32 & -0.01265 & -0.95405 \\
\hline$y-112$ & $y-$ & 112 & 13405.43 & 13405.39 & -0.04674 & -3.48687 \\
\hline$y-113$ & $y-$ & 113 & 13520.46 & 13520.45 & -0.01667 & -1.23294 \\
\hline x114 & $x$ & 114 & 13660.53 & 13660.54 & 0.002497 & 0.182789 \\
\hline$x+117$ & $x+$ & 117 & 14002.74 & 14002.68 & -0.05707 & -4.07563 \\
\hline x118 & $x$ & 118 & 14114.81 & 14114.8 & -0.01071 & -0.75856 \\
\hline$x+120$ & $x+$ & 120 & 14319.88 & 14319.82 & -0.05831 & -4.0721 \\
\hline$y-121$ & $y-$ & 121 & 14420.92 & 14421.03 & 0.107392 & 7.446959 \\
\hline x123 & $x$ & 123 & 14619.96 & 14619.99 & 0.02576 & 1.761975 \\
\hline z124 & $z$ & 124 & 14691.05 & 14691.03 & -0.01845 & -1.25621 \\
\hline y124 & y & 124 & 14707.06 & 14707.08 & 0.01447 & 0.983881 \\
\hline z125 & $z$ & 125 & 14790.11 & 14790.09 & -0.02614 & -1.76746 \\
\hline x126 & $x$ & 126 & 14931.18 & 14931.2 & 0.023141 & 1.549844 \\
\hline$x+127$ & $x+$ & 127 & 15060.25 & 15060.25 & 0.000926 & 0.061484 \\
\hline$y-128$ & $y-$ & 128 & 15160.35 & 15160.35 & -0.00024 & -0.01596 \\
\hline x134 & $x$ & 134 & 15950.68 & 15950.68 & -0.0017 & -0.10652 \\
\hline x135 & $x$ & 135 & 16051.73 & 16051.8 & 0.068588 & 4.272935 \\
\hline$x+136$ & $x+$ & 136 & 16149.79 & 16149.78 & -0.01154 & -0.71469 \\
\hline$x+137$ & $x+$ & 137 & 16264.82 & 16264.77 & -0.04567 & -2.8079 \\
\hline z139 & z & 139 & 16513.92 & 16513.92 & -0.00345 & -0.20922 \\
\hline x139 & $x$ & 139 & 16555.92 & 16555.98 & 0.066074 & 3.99096 \\
\hline x141 & $x$ & 141 & 16770.01 & 16770.03 & 0.012228 & 0.729159 \\
\hline y144 & y & 144 & 17142.2 & 17142.21 & 0.005813 & 0.339105 \\
\hline
\end{tabular}




$\begin{array}{llrrrrr}x+145 & x+ & 145 & 17297.25 & 17297.22 & -0.03156 & -1.82434 \\ x 146 & x & 146 & 17409.33 & 17409.34 & 0.013216 & 0.759133 \\ z 147 & z & 147 & 17480.41 & 17480.35 & -0.06316 & -3.61307 \\ z 149 & z & 149 & 17721.55 & 17721.53 & -0.02196 & -1.23917 \\ \text { z150 } & z & 150 & 17822.6 & 17822.56 & -0.03961 & -2.22251 \\ \text { z152 } & z & 152 & 18006.72 & 18006.69 & -0.03837 & -2.13065 \\ x 154 & x & 154 & 18263.85 & 18263.85 & -0.00211 & -0.11553 \\ x+156 & x+ & 156 & 18420.95 & 18420.95 & 0.007129 & 0.387003 \\ y-157 & y- & 157 & 18449.97 & 18449.95 & -0.01907 & -1.03388 \\ x+157 & x+ & 157 & 18477.97 & 18477.96 & -0.01176 & -0.63616 \\ z 158 & z & 158 & 18534.03 & 18534.05 & 0.0237 & 1.278729 \\ y 159 & y & 159 & 18621.09 & 18621.03 & -0.05341 & -2.86852 \\ y 165 & y & 165 & 19216.49 & 19216.52 & 0.024517 & 1.275831 \\ x+165 & x+ & 165 & 19243.48 & 19243.57 & 0.093871 & 4.878066 \\ y-165 & y- & 165 & 19215.48 & 19215.58 & 0.100505 & 5.230419 \\ z 166 & z & 166 & 19363.54 & 19363.61 & 0.071552 & 3.695193\end{array}$


Table S5C. Summary of UVPD mass spectrum for G12V•GDP complex: fragment ions incorporating mass shift of +462.9781 Da (GDP)

\begin{tabular}{|c|c|c|c|c|c|c|}
\hline & $\underline{\text { Ion }}$ & & Theoretical & Experimental & & $\mathrm{ppm}$ \\
\hline Ion & Type & Residue & Mass & Mass & $\Delta$ mass & error \\
\hline a1 & a & 1 & 492.0046 & 492 & -0.00464 & -9.44097 \\
\hline b12 & $b$ & 12 & 1710.636 & 1710.647 & 0.010428 & 6.095977 \\
\hline 14 & b & 14 & 1866.726 & 1866.718 & -0.00864 & -4.62896 \\
\hline$a+24$ & $a+$ & 24 & 2850.352 & 2850.363 & 0.011669 & 4.09387 \\
\hline a28 & a & 28 & 3341.588 & 3341.589 & 0.000356 & 0.106536 \\
\hline b30 & $b$ & 30 & 3615.72 & 3615.739 & 0.019337 & 5.348036 \\
\hline a48 & $a$ & 48 & 5753.737 & 5753.746 & 0.008745 & 1.519882 \\
\hline$a+106$ & $a+$ & 106 & 12519.96 & 12520.05 & 0.094471 & 7.545629 \\
\hline a108 & $a$ & 108 & 12735.03 & 12735 & -0.02622 & -2.05928 \\
\hline$a+117$ & $a+$ & 117 & 13660.51 & 13660.54 & 0.027427 & 2.007756 \\
\hline$a+122$ & $a+$ & 122 & 14216.78 & 14216.8 & 0.028607 & 2.012198 \\
\hline b129 & $b$ & 129 & 15031.18 & 15031.22 & 0.03799 & 2.527413 \\
\hline a131 & a & 131 & 15202.28 & 15202.36 & 0.076131 & 5.007866 \\
\hline$a+133$ & $a+$ & 133 & 15446.38 & 15446.42 & 0.047054 & 3.046279 \\
\hline c134 & c & 134 & 15603.47 & 15603.5 & 0.021811 & 1.39783 \\
\hline b139 & $b$ & 139 & 16120.7 & 16120.82 & 0.120461 & 7.472441 \\
\hline a139 & a & 139 & 16092.71 & 16092.86 & 0.148629 & 9.235798 \\
\hline c140 & c & 140 & 16250.81 & 16250.79 & -0.02481 & -1.52663 \\
\hline a141 & $a$ & 141 & 16302.84 & 16302.8 & -0.04755 & -2.91667 \\
\hline$a+144$ & $a+$ & 144 & 16693.05 & 16692.98 & -0.06949 & -4.16305 \\
\hline c148 & c & 148 & 17124.27 & 17124.17 & -0.10472 & -6.11541 \\
\hline b149 & $b$ & 149 & 17208.29 & 17208.21 & -0.08362 & -4.8594 \\
\hline a152 & $a$ & 152 & 17521.48 & 17521.45 & -0.03153 & -1.79939 \\
\hline a159 & $a$ & 159 & 18332.82 & 18332.84 & 0.025353 & 1.38293 \\
\hline a162 & $\mathrm{a}$ & 162 & 18701.07 & 18701.03 & -0.03848 & -2.05758 \\
\hline c164 & c & 164 & 18988.22 & 18988.26 & 0.04358 & 2.295107 \\
\hline a164 & $a$ & 164 & 18943.2 & 18943.34 & 0.139803 & 7.380116 \\
\hline b164 & $b$ & 164 & 18971.19 & 18971.36 & 0.167105 & 8.808355 \\
\hline c166 & c & 166 & 19272.42 & 19272.55 & 0.137165 & 7.117167 \\
\hline c167 & c & 167 & 19409.47 & 19409.64 & 0.170052 & 8.761288 \\
\hline z48 & $z$ & 48 & 5998.888 & 5998.901 & 0.013412 & 2.235748 \\
\hline$y-48$ & $y-$ & 48 & 6013.899 & 6013.916 & 0.017121 & 2.84691 \\
\hline$y-51$ & $y-$ & 51 & 6339.063 & 6339.046 & -0.01695 & -2.67358 \\
\hline z56 & $z$ & 56 & 6825.289 & 6825.263 & -0.02597 & -3.80497 \\
\hline z67 & $z$ & 67 & 8037.895 & 8037.945 & 0.050255 & 6.252259 \\
\hline$x+83$ & $x+$ & 83 & 10148.96 & 10148.96 & 0.001795 & 0.176862 \\
\hline$x+88$ & $x+$ & 88 & 10708.24 & 10708.28 & 0.044864 & 4.189669 \\
\hline z91 & $z$ & 91 & 10980.39 & 10980.38 & -0.01175 & -1.07 \\
\hline
\end{tabular}




\begin{tabular}{|c|c|c|c|c|c|c|}
\hline x92 & $x$ & 92 & 11169.46 & 11169.44 & -0.02054 & -1.83894 \\
\hline z95 & z & 95 & 11370.55 & 11370.51 & -0.03337 & -2.93486 \\
\hline$x+102$ & $x+$ & 102 & 12363.99 & 12364.02 & 0.026663 & 2.156502 \\
\hline y103 & $y$ & 103 & 12468.04 & 12468.06 & 0.020138 & 1.615169 \\
\hline$x+106$ & $x+$ & 106 & 12816.16 & 12816.15 & -0.01837 & -1.43311 \\
\hline z110 & $z$ & 110 & 13216.32 & 13216.31 & -0.01172 & -0.88648 \\
\hline$y-114$ & $y-$ & 114 & 13631.53 & 13631.5 & -0.02777 & -2.03733 \\
\hline z114 & $z$ & 114 & 13616.52 & 13616.5 & -0.01456 & -1.06929 \\
\hline$x+119$ & $x+$ & 119 & 14216.81 & 14216.8 & -0.00895 & -0.6294 \\
\hline$y-122$ & $y^{-}$ & 122 & 14475.93 & 14475.91 & -0.0242 & -1.67174 \\
\hline z122 & $z$ & 122 & 14460.92 & 14460.97 & 0.052658 & 3.641401 \\
\hline$x 124$ & $x$ & 124 & 14731.03 & 14731.02 & -0.00632 & -0.4293 \\
\hline$x+125$ & $x+$ & 125 & 14831.1 & 14831.12 & 0.011436 & 0.77108 \\
\hline y127 & y & 127 & 15031.24 & 15031.22 & -0.0247 & -1.64358 \\
\hline z127 & z & 127 & 15015.23 & 15015.27 & 0.041912 & 2.7913 \\
\hline$x+128$ & $x+$ & 128 & 15186.33 & 15186.37 & 0.043392 & 2.857305 \\
\hline z129 & z & 129 & 15299.42 & 15299.4 & -0.0233 & -1.52287 \\
\hline$y-129$ & $y^{-}$ & 129 & 15314.43 & 15314.49 & 0.053652 & 3.503364 \\
\hline$x+130$ & $x+$ & 130 & 15505.49 & 15505.49 & -0.00362 & -0.23327 \\
\hline x132 & $x$ & 132 & 15706.54 & 15706.56 & 0.018237 & 1.161109 \\
\hline$x+133$ & $x+$ & 133 & 15836.59 & 15836.59 & -0.00654 & -0.41322 \\
\hline$x+135$ & $x+$ & 135 & 16050.72 & 16050.72 & -0.0001 & -0.00623 \\
\hline y136 & $y$ & 136 & 16120.79 & 16120.82 & 0.032616 & 2.023226 \\
\hline y137 & y & 137 & 16235.82 & 16235.83 & 0.014712 & 0.906145 \\
\hline z137 & z & 137 & 16219.8 & 16219.85 & 0.055253 & 3.406516 \\
\hline x138 & $x$ & 138 & 16424.86 & 16424.88 & 0.020748 & 1.263207 \\
\hline z140 & $z$ & 140 & 16626.93 & 16626.93 & -0.00339 & -0.20383 \\
\hline$x+140$ & $x+$ & 140 & 16669.94 & 16670 & 0.061557 & 3.692694 \\
\hline y141 & $y$ & 141 & 16742.02 & 16742.03 & 0.016858 & 1.006928 \\
\hline z142 & $z$ & 142 & 16873.07 & 16873.04 & -0.02742 & -1.62484 \\
\hline$x 142$ & $x$ & 142 & 16915.07 & 16915.05 & -0.01666 & -0.98468 \\
\hline z143 & $z$ & 143 & 17010.13 & 17010.12 & -0.00185 & -0.10894 \\
\hline$x 143$ & $x$ & 143 & 17052.12 & 17052.13 & 0.001582 & 0.092774 \\
\hline z144 & z & 144 & 17124.17 & 17124.17 & -0.0023 & -0.13443 \\
\hline x144 & $x$ & 144 & 17166.17 & 17166.2 & 0.036289 & 2.113984 \\
\hline$y-147$ & $y-$ & 147 & 17493.41 & 17493.41 & -0.00186 & -0.10621 \\
\hline$x+147$ & $x+$ & 147 & 17521.4 & 17521.45 & 0.045952 & 2.622619 \\
\hline z148 & $z$ & 148 & 17606.45 & 17606.45 & -0.00096 & -0.05458 \\
\hline$x+148$ & $x+$ & 148 & 17649.46 & 17649.49 & 0.024481 & 1.387066 \\
\hline$x+151$ & $x+$ & 151 & 17976.68 & 17976.67 & -0.00626 & -0.34823 \\
\hline$x+153$ & $x+$ & 153 & 18134.75 & 18134.75 & 0.007364 & 0.40607 \\
\hline$y-155$ & $y-$ & 155 & 18291.87 & 18291.85 & -0.01474 & -0.80582 \\
\hline$x+158$ & $x+$ & 158 & 18575.02 & 18574.98 & -0.03638 & -1.95849 \\
\hline
\end{tabular}




$\begin{array}{lllllll}\text { y158 } & y & 158 & 18548.03 & 18548.01 & -0.02032 & -1.09564 \\ \text { x159 } & x & 159 & 18645.05 & 18645.02 & -0.02978 & -1.59699 \\ \text { z163 } & z & 163 & 18957.28 & 18957.27 & -0.00819 & -0.43213 \\ \text { x163 } & x & 163 & 18999.28 & 18999.37 & 0.089482 & 4.709758 \\ \text { y-164 } & y- & 164 & 19085.37 & 19085.46 & 0.082159 & 4.304817 \\ \text { y168 } & y & 168 & 19607.63 & 19607.72 & 0.093352 & 4.761004 \\ \text { x+168 } & x+ & 168 & 19634.62 & 19634.72 & 0.106262 & 5.411971\end{array}$


Table S5D. Summary of UVPD mass spectrum for G12V•GDP complex: fragment ions incorporating mass shift of +463.9859 Da (GDP)

\begin{tabular}{|c|c|c|c|c|c|c|}
\hline & $\frac{\text { Ion }}{\text { Tyne }}$ & Residue & $\frac{\text { Theoretical }}{\text { Mass }}$ & Experimental & Amass & $\frac{\mathrm{ppm}}{\text { error }}$ \\
\hline a24 & $\bar{a}$ & 24 & 2850.352 & 2850.363 & 0.011694 & 4.102652 \\
\hline$a+25$ & $a+$ & 25 & 2964.444 & 2964.429 & -0.01403 & -4.73243 \\
\hline b26 & $b$ & 26 & 3119.489 & 3119.513 & 0.023797 & 7.628493 \\
\hline c28 & c & 28 & 3387.617 & 3387.62 & 0.002835 & 0.836871 \\
\hline b29 & $b$ & 29 & 3517.659 & 3517.675 & 0.015656 & 4.450687 \\
\hline c49 & c & 49 & 5856.787 & 5856.811 & 0.02386 & 4.073906 \\
\hline b56 & $b$ & 56 & 6627.14 & 6627.163 & 0.023631 & 3.565792 \\
\hline c58 & c & 58 & 6872.277 & 6872.293 & 0.016079 & 2.33969 \\
\hline a106 & $a$ & 106 & 12519.96 & 12520.05 & 0.094496 & 7.547628 \\
\hline$a+111$ & $a+$ & 111 & 13048.19 & 13048.24 & 0.052418 & 4.01726 \\
\hline a117 & $a$ & 117 & 13660.51 & 13660.54 & 0.027452 & 2.009589 \\
\hline$a+121$ & $a+$ & 121 & 14120.73 & 14120.7 & -0.0273 & -1.93326 \\
\hline a122 & $a$ & 122 & 14216.78 & 14216.8 & 0.028632 & 2.013959 \\
\hline b123 & $b$ & 123 & 14331.8 & 14331.87 & 0.067002 & 4.675057 \\
\hline$a+124$ & $a+$ & 124 & 14460.92 & 14460.97 & 0.055148 & 3.813587 \\
\hline$a+126$ & $a+$ & 126 & 14661.03 & 14660.98 & -0.05608 & -3.82524 \\
\hline$a+127$ & $a+$ & 127 & 14776.06 & 14776.04 & -0.02196 & -1.48646 \\
\hline c128 & $c$ & 128 & 14921.12 & 14921.16 & 0.041108 & 2.755021 \\
\hline$a+129$ & $a+$ & 129 & 15005.2 & 15005.22 & 0.012642 & 0.842506 \\
\hline b130 & $b$ & 130 & 15160.25 & 15160.35 & 0.098808 & 6.517571 \\
\hline a133 & $a$ & 133 & 15446.38 & 15446.42 & 0.047079 & 3.047899 \\
\hline$a+135$ & $a+$ & 135 & 15631.5 & 15631.52 & 0.016414 & 1.050057 \\
\hline b137 & $b$ & 137 & 15901.63 & 15901.63 & 0.007268 & 0.45706 \\
\hline$a+142$ & $a+$ & 142 & 16451.93 & 16451.87 & -0.05385 & -3.27305 \\
\hline b143 & $b$ & 143 & 16592 & 16591.97 & -0.02863 & -1.72553 \\
\hline a144 & $a$ & 144 & 16693.05 & 16692.98 & -0.06947 & -4.16155 \\
\hline b151 & $b$ & 151 & 17493.46 & 17493.41 & -0.05551 & -3.17307 \\
\hline c152 & c & 152 & 17567.51 & 17567.42 & -0.08544 & -4.86381 \\
\hline b153 & $b$ & 153 & 17649.55 & 17649.49 & -0.06554 & -3.71364 \\
\hline c156 & c & 156 & 17967.67 & 17967.66 & -0.00479 & -0.26665 \\
\hline$a+166$ & $a+$ & 166 & 19229.41 & 19229.41 & -0.00303 & -0.15736 \\
\hline b168 & $b$ & 168 & 19521.55 & 19521.7 & 0.150232 & 7.6957 \\
\hline y19 & y & 19 & 2767.224 & 2767.216 & -0.00835 & -3.01855 \\
\hline z49 & $z$ & 49 & 6096.949 & 6096.929 & -0.0198 & -3.24818 \\
\hline z58 & z & 58 & 7038.449 & 7038.482 & 0.032495 & 4.616784 \\
\hline y59 & y & 59 & 7185.508 & 7185.57 & 0.061241 & 8.522849 \\
\hline y71 & $y$ & 71 & 8580.26 & 8580.235 & -0.02484 & -2.89478 \\
\hline z82 & $z$ & 82 & 10005.92 & 10005.93 & 0.015304 & 1.529495 \\
\hline
\end{tabular}




\begin{tabular}{|c|c|c|c|c|c|c|}
\hline x83 & $x$ & 83 & 10148.96 & 10148.96 & 0.00182 & 0.179329 \\
\hline$x+85$ & $x+$ & 85 & 10378.05 & 10378.03 & -0.02295 & -2.2114 \\
\hline$x+86$ & $x+$ & 86 & 10491.14 & 10491.14 & -0.00095 & -0.09046 \\
\hline x88 & $x$ & 88 & 10708.24 & 10708.28 & 0.044889 & 4.192007 \\
\hline$x+90$ & $x+$ & 90 & 10911.32 & 10911.33 & 0.007315 & 0.670402 \\
\hline$x+91$ & $x+$ & 91 & 11024.41 & 11024.38 & -0.02963 & -2.68731 \\
\hline z96 & $z$ & 96 & 11472.6 & 11472.57 & -0.02991 & -2.60699 \\
\hline x102 & $x$ & 102 & 12363.99 & 12364.02 & 0.026688 & 2.158526 \\
\hline$y-103$ & $y-$ & 103 & 12468.04 & 12468.06 & 0.020163 & 1.617177 \\
\hline x106 & $x$ & 106 & 12816.16 & 12816.15 & -0.01834 & -1.43116 \\
\hline y108 & y & 108 & 13048.27 & 13048.24 & -0.02758 & -2.11346 \\
\hline$x+110$ & $x+$ & 110 & 13260.34 & 13260.32 & -0.01268 & -0.95594 \\
\hline y112 & $y$ & 112 & 13405.43 & 13405.39 & -0.04677 & -3.48873 \\
\hline y113 & $y$ & 113 & 13520.46 & 13520.45 & -0.01669 & -1.2348 \\
\hline$x+114$ & $x+$ & 114 & 13660.53 & 13660.54 & 0.002472 & 0.180957 \\
\hline$x+118$ & $x+$ & 118 & 14114.81 & 14114.8 & -0.01073 & -0.76034 \\
\hline z119 & $z$ & 119 & 14174.82 & 14174.79 & -0.02749 & -1.93964 \\
\hline x119 & $x$ & 119 & 14216.81 & 14216.8 & -0.00892 & -0.62764 \\
\hline y121 & $y$ & 121 & 14420.92 & 14421.03 & 0.107367 & 7.445223 \\
\hline$x+123$ & $x+$ & 123 & 14619.96 & 14619.99 & 0.025735 & 1.760263 \\
\hline x125 & $x$ & 125 & 14831.1 & 14831.12 & 0.011461 & 0.772768 \\
\hline$x+126$ & $x+$ & 126 & 14931.18 & 14931.2 & 0.023116 & 1.548167 \\
\hline$y-127$ & $y^{-}$ & 127 & 15031.24 & 15031.22 & -0.02468 & -1.64191 \\
\hline y128 & y & 128 & 15160.35 & 15160.35 & -0.00027 & -0.01761 \\
\hline$x 128$ & $x$ & 128 & 15186.33 & 15186.37 & 0.043417 & 2.858953 \\
\hline x130 & $x$ & 130 & 15505.49 & 15505.49 & -0.00359 & -0.23166 \\
\hline z131 & $z$ & 131 & 15550.52 & 15550.5 & -0.02905 & -1.86791 \\
\hline z132 & z & 132 & 15665.55 & 15665.55 & -0.00228 & -0.14529 \\
\hline x133 & $x$ & 133 & 15836.59 & 15836.59 & -0.00652 & -0.41164 \\
\hline$x+134$ & $x+$ & 134 & 15950.68 & 15950.68 & -0.00172 & -0.10809 \\
\hline x135 & $x$ & 135 & 16050.72 & 16050.72 & $\begin{array}{r}-7.50 \mathrm{E}- \\
05\end{array}$ & -0.00467 \\
\hline$x+135$ & $x+$ & 135 & 16051.73 & 16051.8 & 0.068563 & 4.271375 \\
\hline$y-136$ & $y-$ & 136 & 16120.79 & 16120.82 & 0.032641 & 2.024779 \\
\hline z137 & $z$ & 137 & 16220.81 & 16220.82 & 0.01469 & 0.905627 \\
\hline$y-137$ & $y-$ & 137 & 16235.82 & 16235.83 & 0.014737 & 0.907687 \\
\hline$x+139$ & $x+$ & 139 & 16555.92 & 16555.98 & 0.066049 & 3.989448 \\
\hline x140 & $x$ & 140 & 16669.94 & 16670 & 0.061582 & 3.694195 \\
\hline$x+141$ & $x+$ & 141 & 16770.01 & 16770.03 & 0.012203 & 0.727666 \\
\hline$y-141$ & $y^{-}$ & 141 & 16742.02 & 16742.03 & 0.016883 & 1.008423 \\
\hline$x+146$ & $x+$ & 146 & 17409.33 & 17409.34 & 0.013191 & 0.757696 \\
\hline x147 & $x$ & 147 & 17521.4 & 17521.45 & 0.045977 & 2.624048 \\
\hline x148 & $x$ & 148 & 17649.46 & 17649.49 & 0.024506 & 1.388484 \\
\hline
\end{tabular}




$\begin{array}{llllrrr}z 151 & z & 151 & 17934.68 & 17934.61 & -0.06973 & -3.88789 \\ x 151 & x & 151 & 17976.68 & 17976.67 & -0.00623 & -0.34684 \\ \text { x153 } & x & 153 & 18134.75 & 18134.75 & 0.007389 & 0.40745 \\ \text { x+154 } & x+ & 154 & 18263.85 & 18263.85 & -0.00214 & -0.1169 \\ \text { y157 } & y & 157 & 18449.97 & 18449.95 & -0.0191 & -1.03523 \\ \text { x158 } & x & 158 & 18575.02 & 18574.98 & -0.03635 & -1.95714 \\ \text { y-158 } & y- & 158 & 18548.03 & 18548.01 & -0.0203 & -1.09429 \\ \text { z164 } & z & 164 & 19071.37 & 19071.32 & -0.05269 & -2.76268 \\ \text { y165 } & y & 165 & 19215.48 & 19215.58 & 0.10048 & 5.229116 \\ \text { z167 } & z & 167 & 19491.57 & 19491.66 & 0.09145 & 4.691772 \\ y-168 & y- & 168 & 19607.63 & 19607.72 & 0.093377 & 4.76228 \\ \text { x168 } & x & 168 & 19634.62 & 19634.72 & 0.106287 & 5.413245\end{array}$


Table S6A. Summary of UVPD mass spectrum for G12V•GDPnP complex: apo fragment ions

Column "Ions" is the fragment ion identity $(a, b, c, x, y, z)$ along with the number of residues in the fragment ion. A plus or minus sign indicates the fragment ion has one additional hydrogen atom or one less hydrogen atom than a conventional fragment ion. Column "Ion Type" is the type of fragment ion $(a, b, c, x, y, z)$. A plus sign indicates a fragment ion $(a, x)$ with an additional hydrogen atom; a minus sign indicates a fragment ion $(y)$ that is missing one hydrogen atom (the addition of one hydrogen atom for $a / x$ ions is not uncommon in UVPD mass spectra of intact proteins); Column "Residue" is the number of residues in the fragment ion. Column "Theoretical Mass" is the theoretical mass of the fragment ion; Column "Experimental Mass" is the experimentally measured mass of the fragment ion. Column "Delta mass" is the mass difference between the theoretical and experimental masses; and column "ppm error" is the mass error in ppm. The "apo" table (Table S6A) provides the fragment ions that do not retain the ligand. Tables S6B, S6C, and S6D provide the fragment ions that incorporate the ligand based on the mass shift corresponding to the retained ligand (with or without an additional hydrogen atom).

\begin{tabular}{|c|c|c|c|c|c|c|}
\hline & $\underline{\text { Ion }}$ & & Theoretical & Experimental & & $\mathrm{ppm}$ \\
\hline Ion & Type & $\underline{\text { Residue }}$ & Mass & Mass & $\Delta$ mass & error \\
\hline$a+4$ & $a+$ & 4 & 391.1651 & 391.1662 & 0.001025 & 2.620295 \\
\hline a4 & a & 4 & 390.1573 & 390.1583 & 0.001037 & 2.657902 \\
\hline b4 & $b$ & 4 & 418.1522 & 418.1533 & 0.001117 & 2.671276 \\
\hline$c 4$ & c & 4 & 435.1785 & 435.1799 & 0.001368 & 3.143538 \\
\hline a5 & a & 5 & 553.2206 & 553.2222 & 0.001581 & 2.857811 \\
\hline b5 & $b$ & 5 & 581.2156 & 581.2172 & 0.001661 & 2.857804 \\
\hline$a+5$ & $a+$ & 5 & 554.2285 & 554.2304 & 0.001934 & 3.489478 \\
\hline c5 & c & 5 & 598.2419 & 598.2438 & 0.001972 & 3.296326 \\
\hline$a+6$ & $a+$ & 6 & 682.3234 & 682.3254 & 0.002006 & 2.939908 \\
\hline b7 & $b$ & 7 & 822.3946 & 822.3971 & 0.002572 & 3.127453 \\
\hline$a+7$ & $a+$ & 7 & 795.4075 & 795.4101 & 0.002663 & 3.347929 \\
\hline a8 & a & 8 & 893.4681 & 893.4707 & 0.002625 & 2.93799 \\
\hline b8 & $b$ & 8 & 921.463 & 921.4658 & 0.002827 & 3.067947 \\
\hline$a+8$ & $a+$ & 8 & 894.4759 & 894.4791 & 0.003161 & 3.533877 \\
\hline c8 & c & 8 & 938.4893 & 938.4925 & 0.003199 & 3.40867 \\
\hline b9 & $b$ & 9 & 1020.531 & 1020.534 & 0.002959 & 2.89947 \\
\hline$a+9$ & $a+$ & 9 & 993.5443 & 993.5473 & 0.002989 & 3.008389 \\
\hline c9 & c & 9 & 1037.558 & 1037.561 & 0.003393 & 3.27018 \\
\hline c10 & c & 10 & 1136.626 & 1136.629 & 0.002976 & 2.618275 \\
\hline$a+10$ & $a+$ & 10 & 1092.613 & 1092.616 & 0.003549 & 3.248148 \\
\hline b10 & $b$ & 10 & 1119.6 & 1119.604 & 0.004374 & 3.906753 \\
\hline$a+11$ & $a+$ & 11 & 1149.634 & 1149.637 & 0.002718 & 2.364202 \\
\hline b11 & $b$ & 11 & 1176.621 & 1176.625 & 0.003299 & 2.803791 \\
\hline c11 & c & 11 & 1193.648 & 1193.652 & 0.004831 & 4.047258 \\
\hline$a+12$ & $a+$ & 12 & 1220.671 & 1220.674 & 0.002596 & 2.126672 \\
\hline a12 & a & 12 & 1219.663 & 1219.666 & 0.002974 & 2.438378 \\
\hline
\end{tabular}




\begin{tabular}{|c|c|c|c|c|c|c|}
\hline c12 & c & 12 & 1264.685 & 1264.688 & 0.003122 & 2.4686 \\
\hline b12 & $b$ & 12 & 1247.658 & 1247.662 & 0.003177 & 2.54637 \\
\hline c13 & c & 13 & 1363.753 & 1363.755 & 0.001484 & 1.088174 \\
\hline$a+13$ & $a+$ & 13 & 1319.74 & 1319.742 & 0.002545 & 1.928386 \\
\hline b13 & $b$ & 13 & 1346.727 & 1346.73 & 0.003126 & 2.321184 \\
\hline$a+14$ & $a+$ & 14 & 1376.761 & 1376.764 & 0.002692 & 1.95529 \\
\hline c14 & c & 14 & 1420.775 & 1420.778 & 0.003584 & 2.522568 \\
\hline b14 & $b$ & 14 & 1403.748 & 1403.753 & 0.004615 & 3.287627 \\
\hline b15 & $b$ & 15 & 1502.817 & 1502.819 & 0.002611 & 1.737404 \\
\hline$a+15$ & $a+$ & 15 & 1475.83 & 1475.833 & 0.003007 & 2.037476 \\
\hline c15 & c & 15 & 1519.843 & 1519.847 & 0.004143 & 2.72594 \\
\hline$a+16$ & $a+$ & 16 & 1532.851 & 1532.853 & 0.002299 & 1.499799 \\
\hline c16 & c & 16 & 1576.864 & 1576.868 & 0.003313 & 2.101005 \\
\hline b16 & $b$ & 16 & 1559.838 & 1559.842 & 0.003734 & 2.393838 \\
\hline c17 & c & 17 & 1704.959 & 1704.962 & 0.002836 & 1.663383 \\
\hline$a+17$ & $a+$ & 17 & 1660.946 & 1660.95 & 0.003653 & 2.19933 \\
\hline a17 & $a$ & 17 & 1659.938 & 1659.942 & 0.003787 & 2.28141 \\
\hline b17 & $b$ & 17 & 1687.933 & 1687.939 & 0.005576 & 3.303449 \\
\hline c18 & c & 18 & 1791.991 & 1791.991 & -0.00075 & -0.41964 \\
\hline$a+18$ & $a+$ & 18 & 1747.978 & 1747.981 & 0.002606 & 1.490847 \\
\hline$a+19$ & $a+$ & 19 & 1819.015 & 1819.018 & 0.003116 & 1.712997 \\
\hline a19 & $a$ & 19 & 1818.007 & 1818.012 & 0.004885 & 2.687008 \\
\hline b20 & $b$ & 20 & 1959.086 & 1959.089 & 0.002631 & 1.342973 \\
\hline$a+20$ & $a+$ & 20 & 1932.099 & 1932.103 & 0.003642 & 1.88498 \\
\hline c20 & c & 20 & 1976.113 & 1976.117 & 0.004721 & 2.389034 \\
\hline a20 & $a$ & 20 & 1931.091 & 1931.097 & 0.005664 & 2.933056 \\
\hline$a+21$ & $a+$ & 21 & 2033.147 & 2033.132 & -0.01461 & -7.18592 \\
\hline a21 & $a$ & 21 & 2032.139 & 2032.128 & -0.01057 & -5.20092 \\
\hline$a+22$ & $a+$ & 22 & 2146.231 & 2146.234 & 0.002721 & 1.267789 \\
\hline b22 & $b$ & 22 & 2173.218 & 2173.223 & 0.004986 & 2.294294 \\
\hline a22 & a & 22 & 2145.223 & 2145.229 & 0.00576 & 2.685035 \\
\hline$a+23$ & $a+$ & 23 & 2274.29 & 2274.292 & 0.002831 & 1.24477 \\
\hline$a+24$ & $a+$ & 24 & 2387.374 & 2387.377 & 0.003488 & 1.461006 \\
\hline b24 & $b$ & 24 & 2414.361 & 2414.366 & 0.005656 & 2.342649 \\
\hline$a+25$ & $a+$ & 25 & 2500.458 & 2500.46 & 0.002192 & 0.876627 \\
\hline a25 & a & 25 & 2499.45 & 2499.455 & 0.00489 & 1.956431 \\
\hline b25 & $b$ & 25 & 2527.445 & 2527.451 & 0.005825 & 2.304699 \\
\hline$c 25$ & c & 25 & 2544.471 & 2544.477 & 0.00638 & 2.507397 \\
\hline$c 26$ & c & 26 & 2672.53 & 2672.535 & 0.005417 & 2.026919 \\
\hline$a+27$ & $a+$ & 27 & 2742.559 & 2742.562 & 0.002733 & 0.996503 \\
\hline a27 & $\mathrm{a}$ & 27 & 2741.551 & 2741.557 & 0.005675 & 2.069996 \\
\hline$c 27$ & c & 27 & 2786.573 & 2786.579 & 0.006188 & 2.220649 \\
\hline b27 & $b$ & 27 & 2769.546 & 2769.557 & 0.010516 & 3.79701 \\
\hline
\end{tabular}




\begin{tabular}{|c|c|c|c|c|c|c|}
\hline$a+28$ & $a+$ & 28 & 2879.618 & 2879.623 & 0.005346 & 1.856485 \\
\hline c28 & c & 28 & 2923.631 & 2923.638 & 0.00636 & 2.175377 \\
\hline a28 & $a$ & 28 & 2878.61 & 2878.619 & 0.008946 & 3.10775 \\
\hline c29 & c & 29 & 3070.7 & 3070.704 & 0.003868 & 1.259648 \\
\hline$a+29$ & $a+$ & 29 & 3026.686 & 3026.692 & 0.005051 & 1.668811 \\
\hline a29 & $a$ & 29 & 3025.679 & 3025.684 & 0.005722 & 1.891146 \\
\hline$a+30$ & $a+$ & 30 & 3125.755 & 3125.76 & 0.004757 & 1.521862 \\
\hline c30 & c & 30 & 3169.768 & 3169.775 & 0.007236 & 2.282817 \\
\hline a30 & $a$ & 30 & 3124.747 & 3124.757 & 0.010188 & 3.260424 \\
\hline$a+31$ & $a+$ & 31 & 3240.782 & 3240.788 & 0.006137 & 1.893669 \\
\hline b31 & $b$ & 31 & 3267.769 & 3267.779 & 0.010258 & 3.139145 \\
\hline c32 & c & 32 & 3413.838 & 3413.847 & 0.009626 & 2.8197 \\
\hline$a+32$ & $a+$ & 32 & 3369.824 & 3369.838 & 0.013548 & 4.020378 \\
\hline c33 & c & 33 & 3576.901 & 3576.909 & 0.007677 & 2.146271 \\
\hline a33 & $a$ & 33 & 3531.88 & 3531.89 & 0.010485 & 2.968674 \\
\hline b33 & $b$ & 33 & 3559.875 & 3559.899 & 0.024503 & 6.883107 \\
\hline$a+34$ & $a+$ & 34 & 3647.915 & 3647.921 & 0.006822 & 1.870101 \\
\hline$a+35$ & $a+$ & 35 & 3744.967 & 3744.972 & 0.004599 & 1.22804 \\
\hline c35 & $c$ & 35 & 3788.981 & 3788.992 & 0.01074 & 2.834535 \\
\hline a36 & a & 36 & 3845.007 & 3845.014 & 0.006492 & 1.688423 \\
\hline a37 & a & 37 & 3958.091 & 3958.104 & 0.012276 & 3.101495 \\
\hline a38 & a & 38 & 4087.134 & 4087.139 & 0.005574 & 1.363792 \\
\hline a39 & a & 39 & 4202.161 & 4202.152 & -0.0085 & -2.02301 \\
\hline c39 & c & 39 & 4247.182 & 4247.192 & 0.009421 & 2.218177 \\
\hline b39 & $b$ & 39 & 4230.156 & 4230.175 & 0.019608 & 4.63529 \\
\hline$c 40$ & c & 40 & 4334.214 & 4334.174 & -0.04014 & -9.26096 \\
\hline c41 & c & 41 & 4497.277 & 4497.233 & -0.04402 & -9.78837 \\
\hline a41 & a & 41 & 4452.256 & 4452.266 & 0.009787 & 2.198211 \\
\hline a42 & $a$ & 42 & 4608.357 & 4608.369 & 0.011582 & 2.51326 \\
\hline$c 42$ & c & 42 & 4653.379 & 4653.405 & 0.026622 & 5.721005 \\
\hline$a+43$ & $a+$ & 43 & 4737.46 & 4737.469 & 0.009139 & 1.929086 \\
\hline a44 & a & 44 & 4864.511 & 4864.521 & 0.010508 & 2.160135 \\
\hline a45 & a & 45 & 4963.579 & 4963.59 & 0.010946 & 2.205263 \\
\hline a46 & a & 46 & 5062.648 & 5062.658 & 0.010285 & 2.031546 \\
\hline c47 & c & 47 & 5220.753 & 5220.714 & -0.03896 & -7.46253 \\
\hline a47 & a & 47 & 5175.732 & 5175.745 & 0.013261 & 2.56215 \\
\hline b47 & b & 47 & 5203.727 & 5203.747 & 0.020299 & 3.900858 \\
\hline a48 & a & 48 & 5290.759 & 5290.767 & 0.007808 & 1.475781 \\
\hline$a+49$ & $a+$ & 49 & 5348.788 & 5348.794 & 0.006352 & 1.187553 \\
\hline$a+50$ & $a+$ & 50 & 5477.831 & 5477.838 & 0.007341 & 1.340123 \\
\hline c51 & c & 51 & 5622.892 & 5622.854 & -0.03749 & -6.66792 \\
\hline$a+51$ & $a+$ & 51 & 5578.878 & 5578.886 & 0.007513 & 1.346681 \\
\hline b51 & $b$ & 51 & 5605.865 & 5605.889 & 0.02396 & 4.27409 \\
\hline
\end{tabular}




\begin{tabular}{|c|c|c|c|c|c|c|}
\hline a53 & a & 53 & 5793.964 & 5793.966 & 0.002166 & 0.373837 \\
\hline a54 & a & 54 & 5907.048 & 5907.057 & 0.00917 & 1.552383 \\
\hline c55 & c & 55 & 6067.096 & 6067.059 & -0.03711 & -6.11594 \\
\hline a55 & a & 55 & 6022.075 & 6022.077 & 0.002396 & 0.39787 \\
\hline b55 & $b$ & 55 & 6050.07 & 6050.078 & 0.008674 & 1.433703 \\
\hline a56 & $\mathrm{a}$ & 56 & 6135.159 & 6135.173 & 0.01426 & 2.324308 \\
\hline a57 & a & 57 & 6248.243 & 6248.241 & -0.00144 & -0.23062 \\
\hline c58 & c & 58 & 6408.291 & 6408.255 & -0.03636 & -5.67468 \\
\hline a58 & a & 58 & 6363.27 & 6363.259 & -0.01076 & -1.69033 \\
\hline$a+58$ & $a+$ & 58 & 6364.278 & 6364.303 & 0.025561 & 4.016319 \\
\hline c59 & c & 59 & 6509.339 & 6509.302 & -0.03668 & -5.63529 \\
\hline$a+60$ & $a+$ & 60 & 6536.362 & 6536.358 & -0.00454 & -0.69473 \\
\hline a61 & a & 61 & 6592.376 & 6592.391 & 0.014901 & 2.260338 \\
\hline$a 62$ & a & 62 & 6720.435 & 6720.447 & 0.01223 & 1.819823 \\
\hline$a+63$ & $a+$ & 63 & 6850.485 & 6850.491 & 0.005703 & 0.832491 \\
\hline a63 & $a$ & 63 & 6849.477 & 6849.488 & 0.01067 & 1.557783 \\
\hline$a+64$ & $a+$ & 64 & 6979.528 & 6979.538 & 0.010396 & 1.489495 \\
\hline a69 & a & 69 & 7586.794 & 7586.793 & -0.00114 & -0.15079 \\
\hline a70 & a & 70 & 7701.821 & 7701.825 & 0.004142 & 0.537795 \\
\hline$a+71$ & $a+$ & 71 & 7830.887 & 7830.899 & 0.011956 & 1.526771 \\
\hline$c 72$ & c & 72 & 8037.964 & 8037.934 & -0.02999 & -3.73154 \\
\hline a72 & a & 72 & 7992.943 & 7992.949 & 0.006256 & 0.78269 \\
\hline c73 & c & 73 & 8169.004 & 8168.975 & -0.02908 & -3.56004 \\
\hline a73 & a & 73 & 8123.983 & 8123.968 & -0.0147 & -1.80983 \\
\hline$a+74$ & $a+$ & 74 & 8281.092 & 8281.105 & 0.012592 & 1.520569 \\
\hline a75 & a & 75 & 8381.132 & 8381.134 & 0.001716 & 0.204746 \\
\hline a77 & a & 77 & 8567.196 & 8567.215 & 0.019209 & 2.242157 \\
\hline a79 & a & 79 & 8771.286 & 8771.309 & 0.022967 & 2.61843 \\
\hline a80 & a & 80 & 8884.37 & 8884.378 & 0.007754 & 0.872769 \\
\hline a82 & a & 82 & 9086.448 & 9086.474 & 0.026712 & 2.939763 \\
\hline c83 & c & 83 & 9278.537 & 9278.514 & -0.02354 & -2.53671 \\
\hline a83 & a & 83 & 9233.516 & 9233.525 & 0.009083 & 0.983699 \\
\hline$a+84$ & $a+$ & 84 & 9305.561 & 9305.563 & 0.002112 & 0.226958 \\
\hline a85 & $a$ & 85 & 9417.637 & 9417.649 & 0.012059 & 1.28047 \\
\hline a86 & a & 86 & 9531.68 & 9531.674 & -0.0056 & -0.58783 \\
\hline a87 & a & 87 & 9645.723 & 9645.745 & 0.021902 & 2.270644 \\
\hline a88 & $a$ & 88 & 9746.771 & 9746.779 & 0.008157 & 0.836893 \\
\hline a89 & a & 89 & 9874.866 & 9874.884 & 0.018056 & 1.828481 \\
\hline a90 & a & 90 & 9961.898 & 9961.906 & 0.008731 & 0.876439 \\
\hline a91 & a & 91 & 10108.97 & 10108.97 & 0.007582 & 0.750027 \\
\hline a92 & a & 92 & 10238.01 & 10237.99 & -0.01767 & -1.72631 \\
\hline a94 & a & 94 & 10466.12 & 10466.13 & 0.014951 & 1.428514 \\
\hline a96 & a & 96 & 10740.24 & 10740.24 & 0.003261 & 0.303625 \\
\hline
\end{tabular}




\begin{tabular}{|c|c|c|c|c|c|c|}
\hline b169 & $b$ & 169 & 19186.61 & 19186.43 & -0.17403 & -9.07065 \\
\hline a169 & a & 169 & 19158.61 & 19158.52 & -0.09318 & -4.86377 \\
\hline$y-3$ & $y^{-}$ & 3 & 402.2352 & 402.2363 & 0.001004 & 2.496131 \\
\hline y3 & $y$ & 3 & 403.2431 & 403.2441 & 0.001052 & 2.608848 \\
\hline$x 3$ & $x$ & 3 & 429.2223 & 429.2234 & 0.001096 & 2.553455 \\
\hline y4 & y & 4 & 540.302 & 540.3035 & 0.001529 & 2.829899 \\
\hline$x 4$ & $x$ & 4 & 566.2813 & 566.2828 & 0.001573 & 2.777772 \\
\hline $\mathrm{z4}$ & $z$ & 4 & 524.2832 & 524.2851 & 0.001923 & 3.667865 \\
\hline y5 & $y$ & 5 & 668.3969 & 668.399 & 0.002028 & 3.034125 \\
\hline$x+6$ & $x+$ & 6 & 851.4851 & 851.4827 & -0.00249 & -2.92669 \\
\hline$x 6$ & $x$ & 6 & 850.4773 & 850.4798 & 0.002525 & 2.968921 \\
\hline y6 & $y$ & 6 & 824.4981 & 824.5006 & 0.002542 & 3.083088 \\
\hline$x 7$ & $x$ & 7 & 963.5614 & 963.5641 & 0.002693 & 2.79484 \\
\hline y7 & $y$ & 7 & 937.5821 & 937.5849 & 0.002771 & 2.955474 \\
\hline z7 & z & 7 & 921.5634 & 921.5666 & 0.003226 & 3.500573 \\
\hline z8 & $z$ & 8 & 1050.606 & 1050.609 & 0.003361 & 3.199106 \\
\hline y8 & y & 8 & 1066.625 & 1066.628 & 0.003516 & 3.29638 \\
\hline$y-9$ & $y-$ & 9 & 1221.718 & 1221.719 & 0.001174 & 0.960968 \\
\hline y9 & y & 9 & 1222.726 & 1222.728 & 0.002626 & 2.147661 \\
\hline z9 & z & 9 & 1206.707 & 1206.71 & 0.002836 & 2.350198 \\
\hline$x 9$ & $x$ & 9 & 1248.705 & 1248.708 & 0.002975 & 2.382468 \\
\hline$x+10$ & $x+$ & 10 & 1348.781 & 1348.781 & -0.00026 & -0.19427 \\
\hline y10 & y & 10 & 1321.794 & 1321.798 & 0.003552 & 2.687256 \\
\hline$x+11$ & $x+$ & 11 & 1461.865 & 1461.866 & 0.000395 & 0.270181 \\
\hline$y-11$ & $y-$ & 11 & 1433.87 & 1433.872 & 0.00117 & 0.815996 \\
\hline y11 & $y$ & 11 & 1434.878 & 1434.883 & 0.004331 & 3.018374 \\
\hline z12 & $z$ & 12 & 1519.907 & 1519.907 & 7.40E-05 & 0.048687 \\
\hline y12 & y & 12 & 1535.926 & 1535.929 & 0.003526 & 2.295684 \\
\hline$x 12$ & $x$ & 12 & 1561.905 & 1561.91 & 0.005021 & 3.214664 \\
\hline$y-13$ & $y^{-}$ & 13 & 1697.981 & 1697.982 & 0.000755 & 0.444664 \\
\hline z13 & $z$ & 13 & 1682.971 & 1682.973 & 0.002199 & 1.306618 \\
\hline y13 & y & 13 & 1698.989 & 1698.994 & 0.004893 & 2.879948 \\
\hline$x 13$ & $x$ & 13 & 1724.969 & 1724.974 & 0.0059 & 3.420352 \\
\hline z14 & $z$ & 14 & 1830.039 & 1830.043 & 0.003636 & 1.986843 \\
\hline$x 14$ & $x$ & 14 & 1872.037 & 1872.042 & 0.005362 & 2.86426 \\
\hline y14 & $y$ & 14 & 1846.058 & 1846.064 & 0.006721 & 3.640731 \\
\hline$x+15$ & $x+$ & 15 & 1944.082 & 1944.081 & -0.00044 & -0.2248 \\
\hline z15 & $z$ & 15 & 1901.076 & 1901.079 & 0.003025 & 1.591204 \\
\hline y15 & y & 15 & 1917.095 & 1917.101 & 0.005988 & 3.123476 \\
\hline z16 & $z$ & 16 & 2016.103 & 2016.106 & 0.003306 & 1.639797 \\
\hline y16 & y & 16 & 2032.122 & 2032.128 & 0.006711 & 3.30246 \\
\hline$x+17$ & $x+$ & 17 & 2174.136 & 2174.138 & 0.001761 & 0.809962 \\
\hline y18 & $y$ & 18 & 2246.217 & 2246.223 & 0.005891 & 2.622632 \\
\hline
\end{tabular}




\begin{tabular}{|c|c|c|c|c|c|c|}
\hline$y-20$ & $y^{-}$ & 20 & 2430.289 & 2430.294 & 0.004232 & 1.74137 \\
\hline z21 & $z$ & 21 & 2571.379 & 2571.384 & 0.004835 & 1.880314 \\
\hline y22 & $y$ & 22 & 2688.446 & 2688.451 & 0.00504 & 1.874689 \\
\hline$x+23$ & $x+$ & 23 & 2843.528 & 2843.526 & -0.00226 & -0.79304 \\
\hline y23 & $y$ & 23 & 2816.541 & 2816.549 & 0.007784 & 2.763674 \\
\hline y24 & $y$ & 24 & 2887.578 & 2887.586 & 0.008272 & 2.864685 \\
\hline$y-24$ & $y-$ & 24 & 2886.57 & 2886.582 & 0.011458 & 3.969428 \\
\hline$x+25$ & $x+$ & 25 & 3001.597 & 3001.599 & 0.001712 & 0.570352 \\
\hline$x 25$ & $x$ & 25 & 3000.589 & 3000.596 & 0.006498 & 2.165575 \\
\hline$x+26$ & $x+$ & 26 & 3102.645 & 3102.652 & 0.007193 & 2.318334 \\
\hline y26 & y & 26 & 3075.658 & 3075.666 & 0.008467 & 2.752907 \\
\hline$x+27$ & $x+$ & 27 & 3231.687 & 3231.694 & 0.006351 & 1.965217 \\
\hline y27 & $y$ & 27 & 3204.7 & 3204.708 & 0.00731 & 2.281025 \\
\hline z27 & $z$ & 27 & 3188.682 & 3188.69 & 0.008422 & 2.641217 \\
\hline$x+28$ & $x+$ & 28 & 3344.771 & 3344.776 & 0.004567 & 1.365405 \\
\hline$y-28$ & $y-$ & 28 & 3316.777 & 3316.781 & 0.004853 & 1.463177 \\
\hline y28 & $y$ & 28 & 3317.784 & 3317.792 & 0.007404 & 2.23161 \\
\hline z28 & $z$ & 28 & 3301.766 & 3301.774 & 0.008177 & 2.476554 \\
\hline$x+29$ & $x+$ & 29 & 3491.84 & 3491.843 & 0.003492 & 1.000037 \\
\hline$y-29$ & $y-$ & 29 & 3463.845 & 3463.852 & 0.006755 & 1.950154 \\
\hline$y-30$ & $y-$ & 30 & 3560.898 & 3560.903 & 0.005021 & 1.410047 \\
\hline$x+30$ & $x+$ & 30 & 3588.893 & 3588.898 & 0.005419 & 1.509928 \\
\hline$x+31$ & $x+$ & 31 & 3701.977 & 3701.977 & 2.00E-05 & 0.005394 \\
\hline$y-31$ & $y-$ & 31 & 3673.982 & 3673.987 & 0.005433 & 1.478786 \\
\hline z31 & $z$ & 31 & 3658.971 & 3659 & 0.02917 & 7.972187 \\
\hline$x+32$ & $x+$ & 32 & 3758.998 & 3759.002 & 0.004195 & 1.11598 \\
\hline y32 & $y$ & 32 & 3732.011 & 3732.017 & 0.006374 & 1.707926 \\
\hline$x+33$ & $x+$ & 33 & 3922.061 & 3922.066 & 0.004708 & 1.200381 \\
\hline$y-33$ & $y^{-}$ & 33 & 3894.067 & 3894.073 & 0.006093 & 1.564696 \\
\hline$y-34$ & $y-$ & 34 & 3981.099 & 3981.085 & -0.01349 & -3.3875 \\
\hline$x+34$ & $x+$ & 34 & 4009.094 & 4009.095 & 0.001704 & 0.425026 \\
\hline$x+35$ & $x+$ & 35 & 4165.195 & 4165.199 & 0.004502 & 1.080854 \\
\hline$y-35$ & $y-$ & 35 & 4137.2 & 4137.204 & 0.004788 & 1.157312 \\
\hline z35 & $z$ & 35 & 4122.189 & 4122.195 & 0.006573 & 1.594541 \\
\hline y35 & y & 35 & 4138.208 & 4138.214 & 0.006607 & 1.596585 \\
\hline z36 & $z$ & 36 & 4193.226 & 4193.222 & -0.00356 & -0.84875 \\
\hline$y-36$ & $y-$ & 36 & 4208.237 & 4208.239 & 0.00198 & 0.470513 \\
\hline y37 & $y$ & 37 & 4322.329 & 4322.323 & -0.00568 & -1.31318 \\
\hline z37 & $z$ & 37 & 4306.31 & 4306.325 & 0.014798 & 3.436353 \\
\hline y38 & y & 38 & 4437.356 & 4437.366 & 0.010352 & 2.332921 \\
\hline x38 & $x$ & 38 & 4463.335 & 4463.353 & 0.018436 & 4.130544 \\
\hline$y-39$ & $y^{-}$ & 39 & 4564.406 & 4564.363 & -0.04338 & -9.50397 \\
\hline y39 & $y$ & 39 & 4565.414 & 4565.422 & 0.007314 & 1.602045 \\
\hline
\end{tabular}




$\begin{array}{llrrrrr}x+40 & x+ & 40 & 4663.438 & 4663.442 & 0.003866 & 0.828995 \\ x+41 & x+ & 41 & 4791.497 & 4791.514 & 0.016697 & 3.484708 \\ x+42 & x+ & 42 & 4919.592 & 4919.603 & 0.011337 & 2.304453 \\ y 43 & y & 43 & 4993.653 & 4993.663 & 0.010928 & 2.188378 \\ x+43 & x+ & 43 & 5020.64 & 5020.662 & 0.022739 & 4.529098 \\ x+44 & x+ & 44 & 5135.667 & 5135.683 & 0.016429 & 3.198994 \\ z 45 & z & 45 & 5191.729 & 5191.736 & 0.007201 & 1.387014 \\ x 45 & x & 45 & 5233.727 & 5233.748 & 0.020908 & 3.994859 \\ y-47 & y- & 47 & 5463.889 & 5463.904 & 0.015161 & 2.774769 \\ x+47 & x+ & 47 & 5491.884 & 5491.899 & 0.015625 & 2.845102 \\ x+48 & x+ & 48 & 5578.916 & 5578.886 & -0.03004 & -5.38492 \\ y-49 & y- & 49 & 5647.974 & 5647.988 & 0.014452 & 2.558799 \\ x+50 & x+ & 50 & 5789.053 & 5789.072 & 0.01981 & 3.421971 \\ y-50 & y- & 50 & 5761.058 & 5761.081 & 0.023074 & 4.005173 \\ y 51 & y & 51 & 5877.092 & 5877.116 & 0.023438 & 3.988026 \\ y-52 & y- & 52 & 5979.094 & 5979.109 & 0.015585 & 2.606588 \\ x+52 & x+ & 52 & 6007.089 & 6007.108 & 0.019345 & 3.220357 \\ x+53 & x+ & 53 & 6135.184 & 6135.173 & -0.01072 & -1.7473 \\ x 54 & x & 54 & 6248.219 & 6248.241 & 0.022534 & 3.606468 \\ y 61 & y & 61 & 6917.644 & 6917.642 & -0.00127 & -0.18431\end{array}$


Table S6B. Summary of UVPD mass spectrum for G12V•GDPnP complex: fragment ions incorporating mass shift of +543.9760 Da (GDPnp)

\begin{tabular}{|c|c|c|c|c|c|c|}
\hline & $\underline{\text { Ion }}$ & & Theoretical & Experimental & & $\mathrm{ppm}$ \\
\hline Ion & Type & $\underline{\text { Residue }}$ & $\underline{\text { Mass }}$ & $\underline{\text { Mass }}$ & $\Delta$ mass & error \\
\hline a1 & $\mathrm{a}$ & 1 & 573.0025 & 573 & -0.00255 & -4.44152 \\
\hline b1 & $b$ & 1 & 600.9975 & 601 & 0.00254 & 4.226307 \\
\hline b9 & $b$ & 9 & 1564.507 & 1564.494 & -0.0132 & -8.43844 \\
\hline$a+25$ & $a+$ & 25 & 3044.434 & 3044.46 & 0.026144 & 8.587465 \\
\hline c25 & c & 25 & 3088.447 & 3088.477 & 0.0296 & 9.584105 \\
\hline$a+26$ & $a+$ & 26 & 3172.492 & 3172.51 & 0.018101 & 5.705599 \\
\hline a27 & a & 27 & 3285.527 & 3285.545 & 0.017664 & 5.376306 \\
\hline b48 & b & 48 & 5862.73 & 5862.788 & 0.058083 & 9.90716 \\
\hline c85 & c & 85 & 10006.63 & 10006.72 & 0.088964 & 8.890502 \\
\hline$a+91$ & $a+$ & 91 & 10653.95 & 10654 & 0.05013 & 4.705294 \\
\hline c116 & c & 116 & 13671.48 & 13671.39 & -0.09109 & -6.66256 \\
\hline$a+116$ & $a+$ & 116 & 13627.46 & 13627.41 & -0.05743 & -4.21399 \\
\hline c124 & c & 124 & 14584.92 & 14584.94 & 0.016501 & 1.131374 \\
\hline a125 & a & 125 & 14640.95 & 14640.94 & -0.00777 & -0.53043 \\
\hline b132 & b & 132 & 15439.33 & 15439.41 & 0.0741 & 4.79943 \\
\hline$a+134$ & $a+$ & 134 & 15640.46 & 15640.46 & $\begin{array}{r}-7.60 \mathrm{E}- \\
05\end{array}$ & -0.00486 \\
\hline$a+135$ & $a+$ & 135 & 15711.49 & 15711.47 & -0.02655 & -1.68953 \\
\hline a142 & $\mathrm{a}$ & 142 & 16530.91 & 16530.86 & -0.05304 & -3.20829 \\
\hline b147 & b & 147 & 17060.15 & 17060.13 & -0.01994 & -1.16863 \\
\hline a150 & $a$ & 150 & 17417.4 & 17417.25 & -0.14865 & -8.5348 \\
\hline$a+153$ & $a+$ & 153 & 17702.55 & 17702.41 & -0.14673 & -8.28841 \\
\hline c158 & $c$ & 158 & 18357.79 & 18357.83 & 0.043715 & 2.381278 \\
\hline b160 & b & 160 & 18554.9 & 18554.96 & 0.061078 & 3.291746 \\
\hline a160 & $a$ & 160 & 18526.9 & 18526.96 & 0.064538 & 3.483475 \\
\hline a162 & $a$ & 162 & 18782.07 & 18781.96 & -0.11084 & -5.90148 \\
\hline c162 & c & 162 & 18827.09 & 18827.06 & -0.02963 & -1.5738 \\
\hline b162 & $b$ & 162 & 18810.06 & 18810.05 & -0.01596 & -0.84859 \\
\hline$a+165$ & $a+$ & 165 & 19181.31 & 19181.28 & -0.02261 & -1.17881 \\
\hline c165 & c & 165 & 19225.32 & 19225.33 & 0.008836 & 0.459602 \\
\hline a165 & $a$ & 165 & 19180.3 & 19180.43 & 0.129594 & 6.756621 \\
\hline x4 & $x$ & 4 & 1110.257 & 1110.259 & 0.001465 & 1.319514 \\
\hline$x+9$ & $x+$ & 9 & 1793.689 & 1793.678 & -0.01064 & -5.92914 \\
\hline$y-16$ & $y-$ & 16 & 2575.09 & 2575.088 & -0.0015 & -0.58404 \\
\hline z32 & $z$ & 32 & 4259.968 & 4260 & 0.03171 & 7.443717 \\
\hline$y-45$ & $y^{-}$ & 45 & 5750.716 & 5750.726 & 0.010099 & 1.756135 \\
\hline$x 49$ & $x$ & 49 & 6218.937 & 6218.978 & 0.041382 & 6.654192 \\
\hline$y-52$ & $y-$ & 52 & 6523.07 & 6523.1 & 0.029865 & 4.578371 \\
\hline$x 53$ & $x$ & 53 & 6678.152 & 6678.137 & -0.01456 & -2.18024 \\
\hline
\end{tabular}




\begin{tabular}{|c|c|c|c|c|c|c|}
\hline$y-60$ & $y-$ & 60 & 7361.543 & 7361.616 & 0.072593 & 9.861116 \\
\hline$x+67$ & $x+$ & 67 & 8161.899 & 8161.957 & 0.058748 & 7.197831 \\
\hline z69 & $z$ & 69 & 8403.089 & 8403.169 & 0.080453 & 9.574218 \\
\hline z71 & $z$ & 71 & 8644.231 & 8644.213 & -0.01877 & -2.17174 \\
\hline y74 & y & 74 & 9108.457 & 9108.448 & -0.00905 & -0.99369 \\
\hline$x+75$ & $x+$ & 75 & 9272.503 & 9272.484 & -0.01941 & -2.09297 \\
\hline$x+78$ & $x+$ & 78 & 9637.673 & 9637.721 & 0.04811 & 4.991866 \\
\hline$y-79$ & $y-$ & 79 & 9738.721 & 9738.743 & 0.022152 & 2.274635 \\
\hline$x 86$ & $x$ & 86 & 10570.12 & 10570.21 & 0.088531 & 8.37559 \\
\hline$x+87$ & $x+$ & 87 & 10642.17 & 10642.15 & -0.01573 & -1.47809 \\
\hline y88 & $y$ & 88 & 10762.25 & 10762.23 & -0.01464 & -1.36022 \\
\hline$x+101$ & $x+$ & 101 & 12288.89 & 12288.86 & -0.02674 & -2.17595 \\
\hline$x+110$ & $x+$ & 110 & 13340.33 & 13340.3 & -0.02328 & -1.74539 \\
\hline$x+113$ & $x+$ & 113 & 13627.44 & 13627.41 & -0.03345 & -2.45468 \\
\hline x116 & $x$ & 116 & 13967.63 & 13967.56 & -0.06932 & -4.96283 \\
\hline$x+118$ & $x+$ & 118 & 14194.8 & 14194.77 & -0.03135 & -2.20849 \\
\hline$y-121$ & $y-$ & 121 & 14499.91 & 14499.86 & -0.04922 & -3.39478 \\
\hline$x+122$ & $x+$ & 122 & 14584.92 & 14584.94 & 0.013746 & 0.942478 \\
\hline$x+123$ & $x+$ & 123 & 14699.95 & 14699.97 & 0.016103 & 1.095444 \\
\hline y124 & $y$ & 124 & 14786.05 & 14785.97 & -0.07989 & -5.40307 \\
\hline y125 & y & 125 & 14885.12 & 14885.08 & -0.03697 & -2.48382 \\
\hline$x 125$ & $x$ & 125 & 14911.09 & 14911.08 & -0.0177 & -1.18717 \\
\hline$x 126$ & $x$ & 126 & 15010.16 & 15010.14 & -0.02239 & -1.49172 \\
\hline$x+127$ & $x+$ & 127 & 15139.23 & 15139.25 & 0.017649 & 1.165777 \\
\hline x128 & $x$ & 128 & 15266.32 & 15266.3 & -0.01504 & -0.98537 \\
\hline$y-129$ & $y-$ & 129 & 15395.43 & 15395.4 & -0.03482 & -2.26197 \\
\hline y132 & y & 132 & 15761.56 & 15761.49 & -0.06754 & -4.28492 \\
\hline y133 & y & 133 & 15890.6 & 15890.54 & -0.06716 & -4.22627 \\
\hline$y-137$ & $y^{-}$ & 137 & 16315.81 & 16315.76 & -0.04947 & -3.03184 \\
\hline$x+138$ & $x+$ & 138 & 16506.87 & 16506.86 & -0.00207 & -0.12522 \\
\hline z141 & $z$ & 141 & 16807 & 16806.95 & -0.04626 & -2.75248 \\
\hline$y-143$ & $y-$ & 143 & 17106.14 & 17106.08 & -0.05249 & -3.06878 \\
\hline$y-144$ & $y-$ & 144 & 17220.18 & 17220.2 & 0.022549 & 1.309454 \\
\hline z145 & $z$ & 145 & 17333.23 & 17333.15 & -0.07392 & -4.26458 \\
\hline x145 & $x$ & 145 & 17375.22 & 17375.22 & -0.00603 & -0.3471 \\
\hline$y-148$ & $y-$ & 148 & 17702.46 & 17702.41 & -0.05668 & -3.20159 \\
\hline$x+148$ & $x+$ & 148 & 17730.46 & 17730.44 & -0.02181 & -1.22986 \\
\hline$x+149$ & $x+$ & 149 & 17843.54 & 17843.49 & -0.05186 & -2.90654 \\
\hline$x+154$ & $x+$ & 154 & 18343.84 & 18343.79 & -0.04936 & -2.69104 \\
\hline$y-154$ & $y-$ & 154 & 18315.84 & 18315.81 & -0.03394 & -1.85309 \\
\hline z155 & $z$ & 155 & 18357.85 & 18357.83 & -0.02053 & -1.11859 \\
\hline$y-158$ & $y-$ & 158 & 18628.02 & 18627.95 & -0.07794 & -4.18396 \\
\hline$y-161$ & $y-$ & 161 & 18855.15 & 18855.08 & -0.07235 & -3.83715 \\
\hline
\end{tabular}




$\begin{array}{lllllll}x+161 & x+ & 161 & 18883.15 & 18883.08 & -0.06385 & -3.38117 \\ x 165 & x & 165 & 19321.45 & 19321.52 & 0.063146 & 3.268181 \\ z 165 & z & 165 & 19279.46 & 19279.55 & 0.092036 & 4.773786 \\ y-167 & y- & 167 & 19586.57 & 19586.68 & 0.107988 & 5.513371 \\ y 168 & y & 168 & 19688.63 & 19688.74 & 0.111077 & 5.641683 \\ x+168 & x+ & 168 & 19715.61 & 19715.75 & 0.133752 & 6.784063\end{array}$


Table S6C. Summary of UVPD mass spectrum for G12V•GDPnP complex: fragment ions incorporating mass shift of +541.9604 Da (GDPnp)

\begin{tabular}{|c|c|c|c|c|c|c|}
\hline & $\underline{\text { Ion }}$ & & Theoretical & Experimental & & $\mathrm{ppm}$ \\
\hline Ion & Type & $\underline{\text { Residue }}$ & Mass & $\underline{\text { Mass }}$ & $\Delta$ mass & error \\
\hline$a+1$ & $a+$ & 1 & 571.9948 & 572 & 0.00523 & 9.143384 \\
\hline b11 & $b$ & 11 & 1718.582 & 1718.58 & -0.00129 & -0.75062 \\
\hline+21 & $a+$ & 21 & 2575.107 & 2575.088 & -0.01883 & -7.31388 \\
\hline$a+47$ & $a+$ & 47 & 5718.7 & 5718.713 & 0.013494 & 2.359622 \\
\hline$a+49$ & $a+$ & 49 & 5890.748 & 5890.8 & 0.052008 & 8.828754 \\
\hline$a+56$ & $a+$ & 56 & 6678.127 & 6678.137 & 0.01037 & 1.552826 \\
\hline b106 & $b$ & 106 & 12625.93 & 12626 & 0.07118 & 5.637605 \\
\hline b112 & $b$ & 112 & 13284.19 & 13284.28 & 0.088424 & 6.656333 \\
\hline$a+122$ & $a+$ & 122 & 14295.76 & 14295.89 & 0.127118 & 8.892006 \\
\hline b126 & $b$ & 126 & 14765.99 & 14765.97 & -0.02619 & -1.77394 \\
\hline$a+128$ & $a+$ & 128 & 14955.08 & 14955.1 & 0.017135 & 1.145762 \\
\hline b129 & $b$ & 129 & 15110.16 & 15110.2 & 0.038845 & 2.570786 \\
\hline b131 & $b$ & 131 & 15309.26 & 15309.33 & 0.065469 & 4.276431 \\
\hline a137 & a & 137 & 15951.6 & 15951.54 & -0.06235 & -3.90882 \\
\hline c138 & c & 138 & 16159.69 & 16159.65 & -0.03806 & -2.35543 \\
\hline$a+138$ & $a+$ & 138 & 16115.68 & 16115.67 & -0.00539 & -0.33421 \\
\hline b139 & $b$ & 139 & 16199.68 & 16199.72 & 0.037895 & 2.339243 \\
\hline a139 & a & 139 & 16171.69 & 16171.77 & 0.080392 & 4.971157 \\
\hline$a+140$ & $a+$ & 140 & 16285.78 & 16285.83 & 0.045636 & 2.802197 \\
\hline a144 & a & 144 & 16771.02 & 16770.92 & -0.10018 & -5.97316 \\
\hline a152 & $a$ & 152 & 17600.46 & 17600.39 & -0.07393 & -4.20069 \\
\hline$a+156$ & $a+$ & 156 & 18001.63 & 18001.6 & -0.02466 & -1.36971 \\
\hline$a+166$ & $a+$ & 166 & 19307.38 & 19307.37 & -0.01511 & -0.78234 \\
\hline$a+167$ & $a+$ & 167 & 19444.44 & 19444.42 & -0.02338 & -1.20225 \\
\hline c167 & c & 167 & 19488.46 & 19488.64 & 0.177986 & 9.132893 \\
\hline$x 7$ & $x$ & 7 & 1505.522 & 1505.515 & -0.00684 & -4.54328 \\
\hline z16 & $z$ & 16 & 2558.063 & 2558.074 & 0.010632 & 4.156269 \\
\hline$x+48$ & $x+$ & 48 & 6120.876 & 6120.928 & 0.051625 & 8.434245 \\
\hline$x+50$ & $x+$ & 50 & 6331.013 & 6331.016 & 0.002695 & 0.425677 \\
\hline z72 & $z$ & 72 & 8771.258 & 8771.309 & 0.050472 & 5.754248 \\
\hline$x+119$ & $x+$ & 119 & 14295.8 & 14295.89 & 0.089563 & 6.264987 \\
\hline x120 & $x$ & 120 & 14395.84 & 14395.94 & 0.104639 & 7.268699 \\
\hline$x 121$ & $x$ & 121 & 14524.88 & 14524.84 & -0.0378 & -2.60271 \\
\hline y123 & y & 123 & 14670.95 & 14670.93 & -0.01542 & -1.05106 \\
\hline$x+124$ & $x+$ & 124 & 14811.02 & 14811.08 & 0.062145 & 4.195861 \\
\hline z126 & z & 126 & 14966.15 & 14966.09 & -0.05661 & -3.78274 \\
\hline$y-126$ & $y-$ & 126 & 14981.16 & 14981.12 & -0.03703 & -2.4721 \\
\hline y127 & $y$ & 127 & 15110.23 & 15110.2 & -0.02385 & -1.5784 \\
\hline
\end{tabular}




\begin{tabular}{|c|c|c|c|c|c|c|}
\hline x129 & $x$ & 129 & 15420.4 & 15420.43 & 0.028304 & 1.83549 \\
\hline$x+131$ & $x+$ & 131 & 15671.51 & 15671.47 & -0.03772 & -2.40705 \\
\hline$x+133$ & $x+$ & 133 & 15915.57 & 15915.63 & 0.055346 & 3.477472 \\
\hline y134 & $y$ & 134 & 16001.67 & 16001.62 & -0.04854 & -3.03368 \\
\hline$x+135$ & $x+$ & 135 & 16129.71 & 16129.68 & -0.02244 & -1.39116 \\
\hline x135 & $x$ & 135 & 16128.7 & 16128.86 & 0.159994 & 9.919833 \\
\hline y136 & $y$ & 136 & 16199.77 & 16199.72 & -0.04995 & -3.08338 \\
\hline z139 & $z$ & 139 & 16590.89 & 16590.84 & -0.04957 & -2.98772 \\
\hline$x+139$ & $x+$ & 139 & 16633.89 & 16633.96 & 0.065182 & 3.918624 \\
\hline$x+140$ & $x+$ & 140 & 16748.92 & 16748.96 & 0.037265 & 2.224918 \\
\hline z140 & $z$ & 140 & 16705.91 & 16705.96 & 0.044097 & 2.639604 \\
\hline y141 & $y$ & 141 & 16821 & 16820.98 & -0.02208 & -1.31276 \\
\hline$y-143$ & $y-$ & 143 & 17104.12 & 17104 & -0.11989 & -7.00942 \\
\hline x143 & $x$ & 143 & 17131.11 & 17131.03 & -0.07984 & -4.66047 \\
\hline x144 & $x$ & 144 & 17245.15 & 17245.12 & -0.03048 & -1.76769 \\
\hline$x+147$ & $x+$ & 147 & 17600.38 & 17600.39 & 0.003546 & 0.201471 \\
\hline$x+148$ & $x+$ & 148 & 17728.44 & 17728.47 & 0.02973 & 1.676964 \\
\hline$y-149$ & $y-$ & 149 & 17813.53 & 17813.51 & -0.02548 & -1.43032 \\
\hline z149 & $z$ & 149 & 17798.52 & 17798.5 & -0.01942 & -1.09121 \\
\hline z152 & $z$ & 152 & 18083.69 & 18083.64 & -0.0491 & -2.71543 \\
\hline$x 152$ & $x$ & 152 & 18125.69 & 18125.66 & -0.02715 & -1.49804 \\
\hline$x+153$ & $x+$ & 153 & 18213.73 & 18213.73 & 0.005777 & 0.317177 \\
\hline y156 & $y$ & 156 & 18470.93 & 18470.89 & -0.03596 & -1.94674 \\
\hline$x+157$ & $x+$ & 157 & 18554.93 & 18554.96 & 0.022143 & 1.193374 \\
\hline$y-157$ & $y-$ & 157 & 18526.94 & 18526.96 & 0.025603 & 1.381935 \\
\hline y159 & $y$ & 159 & 18698.05 & 18697.99 & -0.06455 & -3.45218 \\
\hline$x+160$ & $x+$ & 160 & 18782.06 & 18781.96 & -0.10215 & -5.43881 \\
\hline z160 & $z$ & 160 & 18739.06 & 18739.1 & 0.040666 & 2.17012 \\
\hline z161 & $z$ & 161 & 18838.12 & 18837.98 & -0.14078 & -7.4732 \\
\hline$y-164$ & $y-$ & 164 & 19164.36 & 19164.26 & -0.10014 & -5.22532 \\
\hline$x+164$ & $x+$ & 164 & 19192.35 & 19192.42 & 0.072848 & 3.795677 \\
\hline y165 & y & 165 & 19293.46 & 19293.41 & -0.05156 & -2.6723 \\
\hline z168 & z & 168 & 19670.59 & 19670.73 & 0.133723 & 6.798117 \\
\hline
\end{tabular}


Table S6D. Summary of UVPD mass spectrum for G12V•GDPnP complex: fragment ions incorporating mass shift of +542.9526 Da (GDPnp)

\begin{tabular}{|c|c|c|c|c|c|c|}
\hline & Ion & & Theoretical & Experimental & & \\
\hline Ion & Type & Residue & $\underline{\text { Mass }}$ & Mass & $\Delta$ mass & ppm error \\
\hline c1 & c & 1 & 617.0004 & 617 & -0.00036 & -0.58347 \\
\hline a21 & a & 21 & 2575.092 & 2575.088 & -0.00321 & -1.24617 \\
\hline $\mathrm{a} 47$ & a & 47 & 5718.684 & 5718.713 & 0.029119 & 5.091905 \\
\hline a56 & a & 56 & 6678.111 & 6678.137 & 0.025995 & 3.892568 \\
\hline a122 & a & 122 & 14295.74 & 14295.89 & 0.142743 & 9.985001 \\
\hline$a+125$ & $a+$ & 125 & 14640.93 & 14640.94 & 0.007809 & 0.533366 \\
\hline a128 & $a$ & 128 & 14955.07 & 14955.1 & 0.03276 & 2.190562 \\
\hline b137 & $b$ & 137 & 15980.59 & 15980.58 & -0.01046 & -0.65436 \\
\hline a138 & a & 138 & 16115.66 & 16115.67 & 0.010239 & 0.635345 \\
\hline a140 & a & 140 & 16285.77 & 16285.83 & 0.061261 & 3.761628 \\
\hline$a+142$ & $a+$ & 142 & 16530.89 & 16530.86 & -0.03746 & -2.26612 \\
\hline$a+150$ & $a+$ & 150 & 17417.38 & 17417.25 & -0.13308 & -7.64059 \\
\hline b153 & $b$ & 153 & 17728.52 & 17728.47 & -0.04469 & -2.52108 \\
\hline b154 & $b$ & 154 & 17843.54 & 17843.49 & -0.05357 & -3.00209 \\
\hline a156 & a & 156 & 18001.61 & 18001.6 & -0.00903 & -0.50173 \\
\hline$a+160$ & $a+$ & 160 & 18526.88 & 18526.96 & 0.080113 & 4.324147 \\
\hline c161 & c & 161 & 18669.97 & 18670 & 0.030051 & 1.60959 \\
\hline$a+162$ & $a+$ & 162 & 18782.05 & 18781.96 & -0.09527 & -5.07224 \\
\hline$a+165$ & $a+$ & 165 & 19180.28 & 19180.43 & 0.145169 & 7.568657 \\
\hline a166 & $a$ & 166 & 19307.37 & 19307.37 & 0.00052 & 0.026933 \\
\hline b166 & $b$ & 166 & 19335.36 & 19335.37 & 0.003107 & 0.16069 \\
\hline a167 & $a$ & 167 & 19444.43 & 19444.42 & -0.00775 & -0.39867 \\
\hline y16 & $y$ & 16 & 2575.074 & 2575.088 & 0.014071 & 5.464308 \\
\hline z19 & z & 19 & 2830.172 & 2830.172 & 1.10E-05 & 0.003887 \\
\hline y45 & y & 45 & 5750.7 & 5750.726 & 0.025674 & 4.4645 \\
\hline$x+49$ & $x+$ & 49 & 6218.921 & 6218.978 & 0.056957 & 9.158657 \\
\hline x50 & $x$ & 50 & 6330.997 & 6331.016 & 0.01832 & 2.893699 \\
\hline y52 & $y$ & 52 & 6523.054 & 6523.1 & 0.04544 & 6.966062 \\
\hline$x+53$ & $x+$ & 53 & 6678.136 & 6678.137 & 0.001015 & 0.151984 \\
\hline z55 & $z$ & 55 & 6806.195 & 6806.214 & 0.019601 & 2.879876 \\
\hline y79 & y & 79 & 9738.705 & 9738.743 & 0.037727 & 3.873924 \\
\hline z82 & $z$ & 82 & 10084.88 & 10084.96 & 0.076314 & 7.567169 \\
\hline$x+86$ & $x+$ & 86 & 10570.11 & 10570.21 & 0.104106 & 9.849095 \\
\hline z110 & $z$ & 110 & 13296.3 & 13296.29 & -0.01063 & -0.79947 \\
\hline$x+116$ & $x+$ & 116 & 13967.61 & 13967.56 & -0.05374 & -3.84776 \\
\hline x119 & $x$ & 119 & 14295.78 & 14295.89 & 0.105188 & 7.357976 \\
\hline y121 & y & 121 & 14499.89 & 14499.86 & -0.03365 & -2.32064 \\
\hline$y-123$ & $y-$ & 123 & 14670.93 & 14670.93 & 0.000205 & 0.013975 \\
\hline
\end{tabular}




\begin{tabular}{|c|c|c|c|c|c|c|}
\hline$x 124$ & $x$ & 124 & 14811 & 14811.08 & 0.07777 & 5.250826 \\
\hline$x+125$ & $x+$ & 125 & 14911.08 & 14911.08 & -0.00213 & -0.14265 \\
\hline$x+126$ & $x+$ & 126 & 15010.15 & 15010.14 & -0.00682 & -0.45409 \\
\hline$y-127$ & $y-$ & 127 & 15110.21 & 15110.2 & -0.00822 & -0.54433 \\
\hline z127 & $z$ & 127 & 15095.2 & 15095.24 & 0.042998 & 2.848455 \\
\hline$x+128$ & $x+$ & 128 & 15266.3 & 15266.3 & 0.000532 & 0.034846 \\
\hline y129 & $y$ & 129 & 15395.41 & 15395.4 & -0.01925 & -1.25031 \\
\hline$x 131$ & $x$ & 131 & 15671.49 & 15671.47 & -0.0221 & -1.41001 \\
\hline z131 & $z$ & 131 & 15629.49 & 15629.49 & 0.002056 & 0.131546 \\
\hline$x 133$ & $x$ & 133 & 15915.56 & 15915.63 & 0.070971 & 4.459221 \\
\hline$y-134$ & $y-$ & 134 & 16001.66 & 16001.62 & -0.03292 & -2.05722 \\
\hline x135 & $x$ & 135 & 16129.69 & 16129.68 & -0.00681 & -0.42245 \\
\hline$y-136$ & $y-$ & 136 & 16199.76 & 16199.72 & -0.03432 & -2.11886 \\
\hline y137 & $y$ & 137 & 16315.79 & 16315.76 & -0.03389 & -2.07725 \\
\hline x139 & $x$ & 139 & 16633.88 & 16633.96 & 0.080807 & 4.857978 \\
\hline$x 140$ & $x$ & 140 & 16748.9 & 16748.96 & 0.05289 & 3.157819 \\
\hline$y-141$ & $y-$ & 141 & 16820.98 & 16820.98 & -0.00646 & -0.38386 \\
\hline y143 & $y$ & 143 & 17106.12 & 17106.08 & -0.03692 & -2.15829 \\
\hline y144 & y & 144 & 17220.16 & 17220.2 & 0.038124 & 2.213916 \\
\hline$x+145$ & $x+$ & 145 & 17375.21 & 17375.22 & 0.009544 & 0.549287 \\
\hline x147 & $x$ & 147 & 17600.37 & 17600.39 & 0.019171 & 1.089239 \\
\hline z148 & z & 148 & 17686.43 & 17686.35 & -0.07795 & -4.4075 \\
\hline y148 & y & 148 & 17702.45 & 17702.41 & -0.0411 & -2.32177 \\
\hline$x 148$ & $x$ & 148 & 17728.43 & 17728.47 & 0.045355 & 2.558321 \\
\hline x153 & $x$ & 153 & 18213.71 & 18213.73 & 0.021402 & 1.175049 \\
\hline z153 & z & 153 & 18171.71 & 18171.75 & 0.036521 & 2.009772 \\
\hline y154 & y & 154 & 18315.83 & 18315.81 & -0.01837 & -1.00274 \\
\hline$y-156$ & $y-$ & 156 & 18470.91 & 18470.89 & -0.02033 & -1.10081 \\
\hline z157 & $z$ & 157 & 18512.92 & 18512.92 & $\begin{array}{r}-9.10 \mathrm{E}- \\
05\end{array}$ & -0.00492 \\
\hline x157 & $x$ & 157 & 18554.92 & 18554.96 & 0.037768 & 2.035471 \\
\hline y158 & $y$ & 158 & 18628.01 & 18627.95 & -0.06236 & -3.34786 \\
\hline$y-159$ & $y-$ & 159 & 18698.04 & 18697.99 & -0.04892 & -2.61653 \\
\hline x160 & $x$ & 160 & 18782.05 & 18781.96 & -0.08653 & -4.6069 \\
\hline y161 & y & 161 & 18855.13 & 18855.08 & -0.05677 & -3.01112 \\
\hline z164 & $z$ & 164 & 19150.34 & 19150.41 & 0.076655 & 4.002802 \\
\hline x164 & $x$ & 164 & 19192.33 & 19192.42 & 0.088473 & 4.609809 \\
\hline$y-165$ & $y-$ & 165 & 19293.44 & 19293.41 & -0.03593 & -1.86244 \\
\hline$x+165$ & $x+$ & 165 & 19321.44 & 19321.52 & 0.078721 & 4.074281 \\
\hline z166 & $z$ & 166 & 19441.5 & 19441.6 & 0.10484 & 5.392589 \\
\hline y167 & y & 167 & 19586.56 & 19586.68 & 0.123563 & 6.308562 \\
\hline
\end{tabular}




\section{Table S7A. Summary of UVPD mass spectrum for WT•GDP complex: apo fragment ions}

Column "Ions" is the fragment ion identity $(a, b, c, x, y, z)$ along with the number of residues in the fragment ion. A plus or minus sign indicates the fragment ion has one additional hydrogen atom or one less hydrogen atom than a conventional fragment ion. Column "Ion Type" is the type of fragment ion $(a, b, c, x, y, z)$. A plus sign indicates a fragment ion $(a, x)$ with an additional hydrogen atom; a minus sign indicates a fragment ion $(y)$ that is missing one hydrogen atom (the addition of one hydrogen atom for $a / x$ ions is not uncommon in UVPD mass spectra of intact proteins); Column "Residue" is the number of residues in the fragment ion. Column "Theoretical Mass" is the theoretical mass of the fragment ion; Column "Experimental Mass" is the experimentally measured mass of the fragment ion. Column "Delta mass" is the mass difference between the theoretical and experimental masses; and column "ppm error" is the mass error in ppm. The "apo" table (Table S7A) provides the fragment ions that do not retain the ligand. Tables S7B, S7C, and S7D provide the fragment ions that incorporate the ligand based on the mass shift corresponding to the retained ligand (with or without an additional hydrogen atom).

\begin{tabular}{|c|c|c|c|c|c|c|}
\hline & $\underline{\text { Ion }}$ & & Theoretical & Experimental & & ppm \\
\hline Ion & Type & Residue & Mass & Mass & $\Delta$ mass & error \\
\hline b3 & $\mathrm{b}$ & 3 & 289.1096 & 289.1122 & 0.0026 & 8.993128 \\
\hline$a+4$ & $a+$ & 4 & 391.1651 & 391.1686 & 0.003497 & 8.939876 \\
\hline a4 & $a$ & 4 & 390.1573 & 390.1608 & 0.003509 & 8.993808 \\
\hline b4 & b & 4 & 418.1522 & 418.156 & 0.003742 & 8.948894 \\
\hline c4 & c & 4 & 435.1785 & 435.1826 & 0.004114 & 9.453592 \\
\hline$a+5$ & $a+$ & 5 & 554.2285 & 554.2292 & 0.000714 & 1.28822 \\
\hline a5 & a & 5 & 553.2206 & 553.2258 & 0.005182 & 9.366968 \\
\hline b5 & $b$ & 5 & 581.2156 & 581.2211 & 0.005506 & 9.47325 \\
\hline$a+6$ & $a+$ & 6 & 682.3234 & 682.3301 & 0.006706 & 9.828137 \\
\hline b6 & $b$ & 6 & 709.3105 & 709.3175 & 0.006981 & 9.841952 \\
\hline$a+7$ & $a+$ & 7 & 795.4075 & 795.4156 & 0.008156 & 10.25382 \\
\hline b7 & $b$ & 7 & 822.3946 & 822.4028 & 0.008188 & 9.956291 \\
\hline a8 & a & 8 & 893.4681 & 893.4769 & 0.008789 & 9.836949 \\
\hline b8 & $b$ & 8 & 921.463 & 921.4722 & 0.009175 & 9.956993 \\
\hline$a+8$ & $a+$ & 8 & 894.4759 & 894.4851 & 0.009204 & 10.28979 \\
\hline$a+9$ & $a+$ & 9 & 993.5443 & 993.5539 & 0.009581 & 9.643222 \\
\hline b9 & $b$ & 9 & 1020.531 & 1020.541 & 0.009612 & 9.418623 \\
\hline c9 & C & 9 & 1037.558 & 1037.567 & 0.00974 & 9.38743 \\
\hline a9 & a & 9 & 992.5365 & 992.5463 & 0.009776 & 9.849512 \\
\hline a10 & a & 10 & 1091.605 & 1091.616 & 0.010641 & 9.748033 \\
\hline$a+11$ & $a+$ & 11 & 1149.634 & 1149.645 & 0.010897 & 9.47864 \\
\hline b11 & $b$ & 11 & 1176.621 & 1176.633 & 0.011348 & 9.644565 \\
\hline b12 & $b$ & 12 & 1247.658 & 1247.67 & 0.011722 & 9.3952 \\
\hline$a+12$ & $a+$ & 12 & 1220.671 & 1220.683 & 0.011751 & 9.626644 \\
\hline$a+13$ & $a+$ & 13 & 1277.693 & 1277.705 & 0.012264 & 9.598527 \\
\hline c13 & C & 13 & 1321.706 & 1321.719 & 0.012912 & 9.769191 \\
\hline
\end{tabular}




\begin{tabular}{|c|c|c|c|c|c|c|}
\hline a14 & a & 14 & 1333.706 & 1333.719 & 0.012667 & 9.497593 \\
\hline$a+14$ & $a+$ & 14 & 1334.714 & 1334.727 & 0.01302 & 9.754873 \\
\hline c14 & c & 14 & 1378.728 & 1378.741 & 0.01318 & 9.559539 \\
\hline$a+15$ & $a+$ & 15 & 1433.783 & 1433.795 & 0.012482 & 8.705621 \\
\hline b15 & $b$ & 15 & 1460.77 & 1460.783 & 0.013184 & 9.025379 \\
\hline a15 & a & 15 & 1432.775 & 1432.789 & 0.014325 & 9.998082 \\
\hline c15 & c & 15 & 1477.796 & 1477.81 & 0.014472 & 9.792962 \\
\hline b16 & $b$ & 16 & 1517.791 & 1517.803 & 0.011988 & 7.89832 \\
\hline a16 & a & 16 & 1489.796 & 1489.811 & 0.014838 & 9.959751 \\
\hline c16 & c & 16 & 1534.817 & 1534.832 & 0.015033 & 9.79465 \\
\hline a17 & $a$ & 17 & 1617.891 & 1617.906 & 0.01436 & 8.875751 \\
\hline$a+17$ & $a+$ & 17 & 1618.899 & 1618.914 & 0.014674 & 9.064165 \\
\hline c17 & c & 17 & 1662.912 & 1662.928 & 0.01541 & 9.266874 \\
\hline$a+18$ & $a+$ & 18 & 1705.931 & 1705.947 & 0.015568 & 9.12579 \\
\hline$a+19$ & $a+$ & 19 & 1776.968 & 1776.984 & 0.015812 & 8.898284 \\
\hline a19 & $\mathrm{a}$ & 19 & 1775.96 & 1775.978 & 0.017289 & 9.735015 \\
\hline b19 & $b$ & 19 & 1803.955 & 1803.973 & 0.017613 & 9.763546 \\
\hline$a+20$ & $a+$ & 20 & 1890.052 & 1890.068 & 0.015689 & 8.300812 \\
\hline a20 & $\mathrm{a}$ & 20 & 1889.044 & 1889.06 & 0.015701 & 8.31161 \\
\hline$a+21$ & $a+$ & 21 & 1991.1 & 1991.116 & 0.016518 & 8.295901 \\
\hline c21 & c & 21 & 2035.113 & 2035.131 & 0.01741 & 8.554806 \\
\hline$a+22$ & $a+$ & 22 & 2104.184 & 2104.202 & 0.018274 & 8.684587 \\
\hline c22 & c & 22 & 2148.197 & 2148.217 & 0.019776 & 9.205858 \\
\hline$a+23$ & $a+$ & 23 & 2232.243 & 2232.256 & 0.013845 & 6.202269 \\
\hline a23 & $a$ & 23 & 2231.235 & 2231.255 & 0.020009 & 8.96768 \\
\hline b23 & $b$ & 23 & 2259.23 & 2259.25 & 0.020211 & 8.94597 \\
\hline$a+24$ & $a+$ & 24 & 2345.327 & 2345.342 & 0.015723 & 6.703957 \\
\hline$c 24$ & $c$ & 24 & 2389.34 & 2389.361 & 0.021423 & 8.966074 \\
\hline b25 & $b$ & 25 & 2485.398 & 2485.421 & 0.022989 & 9.249626 \\
\hline a25 & a & 25 & 2457.403 & 2457.426 & 0.023276 & 9.471788 \\
\hline b26 & $b$ & 26 & 2613.456 & 2613.479 & 0.023003 & 8.801754 \\
\hline$a+27$ & $a+$ & 27 & 2700.512 & 2700.535 & 0.022339 & 8.272123 \\
\hline b27 & $b$ & 27 & 2727.499 & 2727.524 & 0.024751 & 9.074613 \\
\hline a27 & a & 27 & 2699.504 & 2699.53 & 0.025281 & 9.365052 \\
\hline$c 27$ & c & 27 & 2744.526 & 2744.551 & 0.025551 & 9.309806 \\
\hline$a+28$ & $a+$ & 28 & 2837.571 & 2837.595 & 0.023976 & 8.449469 \\
\hline b28 & $b$ & 28 & 2864.558 & 2864.584 & 0.02613 & 9.121826 \\
\hline a28 & a & 28 & 2836.563 & 2836.59 & 0.02643 & 9.317613 \\
\hline c28 & c & 28 & 2881.584 & 2881.611 & 0.026455 & 9.180713 \\
\hline c29 & c & 29 & 3028.653 & 3028.667 & 0.013879 & 4.582565 \\
\hline$a+29$ & $a+$ & 29 & 2984.64 & 2984.666 & 0.026292 & 8.809093 \\
\hline b29 & $b$ & 29 & 3011.627 & 3011.654 & 0.027667 & 9.18673 \\
\hline$a+30$ & $a+$ & 30 & 3083.708 & 3083.687 & -0.0211 & -6.8408 \\
\hline
\end{tabular}




\begin{tabular}{|c|c|c|c|c|c|c|}
\hline c30 & c & 30 & 3127.721 & 3127.721 & -0.00079 & -0.25354 \\
\hline a30 & a & 30 & 3082.7 & 3082.729 & 0.029306 & 9.506601 \\
\hline b30 & $b$ & 30 & 3110.695 & 3110.725 & 0.029738 & 9.559921 \\
\hline$a+31$ & $a+$ & 31 & 3198.735 & 3198.761 & 0.026231 & 8.20042 \\
\hline c31 & $c$ & 31 & 3242.748 & 3242.776 & 0.028222 & 8.703112 \\
\hline b31 & $b$ & 31 & 3225.722 & 3225.754 & 0.031865 & 9.878409 \\
\hline$a+32$ & $a+$ & 32 & 3327.777 & 3327.805 & 0.027399 & 8.233414 \\
\hline b32 & $b$ & 32 & 3354.765 & 3354.794 & 0.02951 & 8.796445 \\
\hline a32 & a & 32 & 3326.77 & 3326.802 & 0.032041 & 9.631265 \\
\hline c32 & c & 32 & 3371.791 & 3371.823 & 0.032553 & 9.654513 \\
\hline$a+33$ & $a+$ & 33 & 3490.841 & 3490.867 & 0.026024 & 7.454928 \\
\hline c33 & $c$ & 33 & 3534.854 & 3534.884 & 0.029968 & 8.47786 \\
\hline c34 & C & 34 & 3649.881 & 3649.858 & -0.0228 & -6.2476 \\
\hline$a+34$ & $a+$ & 34 & 3605.868 & 3605.896 & 0.027893 & 7.735439 \\
\hline$a+35$ & $a+$ & 35 & 3702.92 & 3702.95 & 0.029088 & 7.855413 \\
\hline c35 & c & 35 & 3746.934 & 3746.967 & 0.033324 & 8.893672 \\
\hline$a+36$ & $a+$ & 36 & 3803.968 & 3803.995 & 0.027306 & 7.178285 \\
\hline a36 & $a$ & 36 & 3802.96 & 3802.989 & 0.028343 & 7.452878 \\
\hline c37 & c & 37 & 3961.066 & 3961.096 & 0.030198 & 7.623706 \\
\hline a37 & a & 37 & 3916.044 & 3916.078 & 0.033736 & 8.614816 \\
\hline a38 & a & 38 & 4045.087 & 4045.121 & 0.033545 & 8.292776 \\
\hline c38 & c & 38 & 4090.108 & 4090.148 & 0.039854 & 9.743997 \\
\hline b38 & $b$ & 38 & 4073.082 & 4073.122 & 0.04052 & 9.948241 \\
\hline c39 & c & 39 & 4205.135 & 4205.124 & -0.01096 & -2.60729 \\
\hline a39 & a & 39 & 4160.114 & 4160.152 & 0.038036 & 9.143019 \\
\hline b39 & $b$ & 39 & 4188.109 & 4188.148 & 0.038726 & 9.246656 \\
\hline$c 40$ & c & 40 & 4292.167 & 4292.147 & -0.02029 & -4.72698 \\
\hline$a+40$ & $a+$ & 40 & 4248.154 & 4248.191 & 0.036802 & 8.66305 \\
\hline a41 & $\mathrm{a}$ & 41 & 4410.209 & 4410.248 & 0.038736 & 8.783257 \\
\hline a42 & a & 42 & 4566.31 & 4566.35 & 0.039855 & 8.728053 \\
\hline$c 42$ & c & 42 & 4611.332 & 4611.377 & 0.04574 & 9.919044 \\
\hline c43 & $\mathrm{C}$ & 43 & 4739.427 & 4739.464 & 0.037084 & 7.824575 \\
\hline$a+43$ & $a+$ & 43 & 4695.413 & 4695.452 & 0.038511 & 8.201827 \\
\hline$a+44$ & $a+$ & 44 & 4823.472 & 4823.51 & 0.038403 & 7.961686 \\
\hline a45 & $a$ & 45 & 4921.532 & 4921.574 & 0.042149 & 8.564203 \\
\hline a46 & $a$ & 46 & 5020.601 & 5020.64 & 0.038924 & 7.752857 \\
\hline$a+47$ & $a+$ & 47 & 5134.693 & 5134.733 & 0.040789 & 7.943799 \\
\hline$a+49$ & $a+$ & 49 & 5306.741 & 5306.781 & 0.040119 & 7.560001 \\
\hline$a+50$ & $a+$ & 50 & 5435.784 & 5435.828 & 0.044404 & 8.168826 \\
\hline c51 & $c$ & 51 & 5580.845 & 5580.867 & 0.021908 & 3.925571 \\
\hline$a+51$ & $a+$ & 51 & 5536.831 & 5536.873 & 0.042012 & 7.587728 \\
\hline a52 & $a$ & 52 & 5638.833 & 5638.876 & 0.042844 & 7.598026 \\
\hline a53 & a & 53 & 5751.917 & 5751.956 & 0.039595 & 6.883792 \\
\hline
\end{tabular}




\begin{tabular}{|c|c|c|c|c|c|c|}
\hline a54 & a & 54 & 5865.001 & 5865.048 & 0.047698 & 8.13265 \\
\hline a55 & a & 55 & 5980.028 & 5980.057 & 0.028936 & 4.838774 \\
\hline$a+56$ & $a+$ & 56 & 6094.12 & 6094.151 & 0.031534 & 5.174491 \\
\hline b56 & $b$ & 56 & 6121.107 & 6121.162 & 0.055476 & 9.063067 \\
\hline a57 & a & 57 & 6206.196 & 6206.241 & 0.044776 & 7.214726 \\
\hline$c 58$ & c & 58 & 6366.244 & 6366.251 & 0.006679 & 1.049127 \\
\hline$a+58$ & $a+$ & 58 & 6322.231 & 6322.272 & 0.041017 & 6.487737 \\
\hline a58 & $\mathrm{a}$ & 58 & 6321.223 & 6321.267 & 0.044007 & 6.961786 \\
\hline c59 & c & 59 & 6467.292 & 6467.295 & 0.00376 & 0.581387 \\
\hline$a+59$ & $a+$ & 59 & 6423.278 & 6423.328 & 0.049245 & 7.666641 \\
\hline a59 & a & 59 & 6422.27 & 6422.329 & 0.058687 & 9.138046 \\
\hline a60 & $a$ & 60 & 6493.308 & 6493.341 & 0.033632 & 5.179487 \\
\hline$a+61$ & $a+$ & 61 & 6551.337 & 6551.37 & 0.0334 & 5.098191 \\
\hline c61 & c & 61 & 6595.35 & 6595.407 & 0.056613 & 8.583775 \\
\hline a62 & $a$ & 62 & 6678.388 & 6678.445 & 0.056992 & 8.533796 \\
\hline a63 & a & 63 & 6807.43 & 6807.488 & 0.058128 & 8.538905 \\
\hline a64 & a & 64 & 6936.473 & 6936.534 & 0.061013 & 8.795969 \\
\hline a65 & a & 65 & 7099.536 & 7099.595 & 0.058474 & 8.236313 \\
\hline$a+66$ & $a+$ & 66 & 7187.576 & 7187.567 & -0.0089 & -1.23756 \\
\hline a69 & $\mathrm{a}$ & 69 & 7544.747 & 7544.806 & 0.058989 & 7.818553 \\
\hline b70 & $b$ & 70 & 7687.769 & 7687.828 & 0.059668 & 7.76142 \\
\hline a70 & $a$ & 70 & 7659.774 & 7659.85 & 0.075995 & 9.921311 \\
\hline$a+71$ & $a+$ & 71 & 7788.84 & 7788.897 & 0.056465 & 7.249471 \\
\hline c72 & $c$ & 72 & 7995.917 & 7995.944 & 0.027108 & 3.39023 \\
\hline a72 & a & 72 & 7950.896 & 7950.961 & 0.064925 & 8.165747 \\
\hline a73 & $a$ & 73 & 8081.936 & 8081.996 & 0.060079 & 7.433738 \\
\hline$a+74$ & $a+$ & 74 & 8239.045 & 8239.113 & 0.068257 & 8.284573 \\
\hline c75 & $c$ & 75 & 8384.106 & 8384.152 & 0.045639 & 5.443514 \\
\hline a75 & a & 75 & 8339.085 & 8339.14 & 0.055101 & 6.607559 \\
\hline$a+76$ & $a+$ & 76 & 8397.114 & 8397.169 & 0.054537 & 6.494727 \\
\hline a77 & a & 77 & 8525.149 & 8525.216 & 0.066763 & 7.8313 \\
\hline$a+78$ & $a+$ & 78 & 8583.178 & 8583.241 & 0.062264 & 7.254186 \\
\hline a79 & $\mathrm{a}$ & 79 & 8729.239 & 8729.314 & 0.075474 & 8.646115 \\
\hline a80 & a & 80 & 8842.323 & 8842.392 & 0.069244 & 7.830974 \\
\hline c82 & c & 82 & 9089.422 & 9089.481 & 0.059649 & 6.562464 \\
\hline$a+82$ & $a+$ & 82 & 9045.408 & 9045.475 & 0.066691 & 7.372908 \\
\hline b82 & $b$ & 82 & 9072.395 & 9072.464 & 0.068127 & 7.509263 \\
\hline b83 & $b$ & 83 & 9219.464 & 9219.545 & 0.080868 & 8.771443 \\
\hline a83 & a & 83 & 9191.469 & 9191.556 & 0.087039 & 9.469542 \\
\hline a84 & a & 84 & 9262.506 & 9262.555 & 0.048464 & 5.232277 \\
\hline b84 & $b$ & 84 & 9290.501 & 9290.577 & 0.075497 & 8.126257 \\
\hline a85 & a & 85 & 9375.59 & 9375.651 & 0.060718 & 6.476179 \\
\hline a87 & $a$ & 87 & 9603.676 & 9603.754 & 0.077885 & 8.109915 \\
\hline
\end{tabular}




\begin{tabular}{|c|c|c|c|c|c|c|}
\hline a89 & a & 89 & 9832.819 & 9832.896 & 0.077701 & 7.90221 \\
\hline$a+90$ & $a+$ & 90 & 9920.859 & 9920.927 & 0.068852 & 6.940122 \\
\hline a91 & $a$ & 91 & 10066.92 & 10067 & 0.081875 & 8.133074 \\
\hline a92 & a & 92 & 10195.96 & 10196.04 & 0.078592 & 7.70815 \\
\hline$a+93$ & $a+$ & 93 & 10312 & 10312.05 & 0.058231 & 5.646915 \\
\hline a94 & $\mathrm{a}$ & 94 & 10424.07 & 10424.16 & 0.082653 & 7.929051 \\
\hline a95 & a & 95 & 10561.13 & 10561.21 & 0.079651 & 7.5419 \\
\hline a96 & a & 96 & 10698.19 & 10698.27 & 0.075185 & 7.027824 \\
\hline a97 & a & 97 & 10861.25 & 10861.33 & 0.076186 & 7.014476 \\
\hline b129 & $b$ & 129 & 14526.16 & 14526.05 & -0.10664 & -7.34103 \\
\hline b130 & $b$ & 130 & 14654.22 & 14654.12 & -0.09393 & -6.40962 \\
\hline b134 & $b$ & 134 & 15081.42 & 15081.33 & -0.08992 & -5.96263 \\
\hline a148 & $a$ & 148 & 16574.23 & 16574.1 & -0.1234 & -7.44523 \\
\hline$a+164$ & $a+$ & 164 & 18439.18 & 18439.06 & -0.12325 & -6.68392 \\
\hline c167 & c & 167 & 18904.45 & 18904.3 & -0.15454 & -8.17464 \\
\hline$a+169$ & $a+$ & 169 & 19117.57 & 19117.58 & 0.005042 & 0.263735 \\
\hline z3 & $z$ & 3 & 387.2243 & 387.2278 & 0.003521 & 9.092921 \\
\hline$y-3$ & $y-$ & 3 & 402.2352 & 402.2388 & 0.003567 & 8.868025 \\
\hline y3 & y & 3 & 403.2431 & 403.2467 & 0.003585 & 8.890419 \\
\hline$x 3$ & $x$ & 3 & 429.2223 & 429.2262 & 0.003812 & 8.881178 \\
\hline $\mathrm{z} 4$ & $z$ & 4 & 524.2832 & 524.2881 & 0.004852 & 9.25454 \\
\hline y4 & $y$ & 4 & 540.302 & 540.307 & 0.005008 & 9.268891 \\
\hline z8 & $z$ & 8 & 1050.606 & 1050.616 & 0.010319 & 9.821951 \\
\hline z9 & $z$ & 9 & 1206.707 & 1206.718 & 0.011137 & 9.229249 \\
\hline$x 9$ & $x$ & 9 & 1248.705 & 1248.716 & 0.011276 & 9.030155 \\
\hline y9 & $y$ & 9 & 1222.726 & 1222.738 & 0.012026 & 9.835402 \\
\hline$x 11$ & $x$ & 11 & 1460.858 & 1460.871 & 0.013874 & 9.497161 \\
\hline y11 & $y$ & 11 & 1434.878 & 1434.892 & 0.013974 & 9.738805 \\
\hline$x 12$ & $x$ & 12 & 1561.905 & 1561.92 & 0.014373 & 9.202223 \\
\hline y12 & $y$ & 12 & 1535.926 & 1535.941 & 0.014699 & 9.570123 \\
\hline z13 & $z$ & 13 & 1682.971 & 1682.986 & 0.015405 & 9.153458 \\
\hline$x+14$ & $x+$ & 14 & 1873.045 & 1873.058 & 0.01365 & 7.287582 \\
\hline z14 & $z$ & 14 & 1830.039 & 1830.056 & 0.017308 & 9.457722 \\
\hline$x+15$ & $x+$ & 15 & 1944.082 & 1944.096 & 0.01426 & 7.335065 \\
\hline z15 & $z$ & 15 & 1901.076 & 1901.093 & 0.017063 & 8.975443 \\
\hline y16 & y & 16 & 2032.122 & 2032.141 & 0.019576 & 9.633281 \\
\hline y17 & $y$ & 17 & 2147.149 & 2147.169 & 0.019857 & 9.248079 \\
\hline$x+18$ & $x+$ & 18 & 2273.204 & 2273.222 & 0.018068 & 7.948238 \\
\hline y21 & $y$ & 21 & 2587.398 & 2587.419 & 0.020697 & 7.999155 \\
\hline$x+21$ & $x+$ & 21 & 2614.385 & 2614.407 & 0.021774 & 8.328523 \\
\hline$y-21$ & $y^{-}$ & 21 & 2586.39 & 2586.413 & 0.022799 & 8.815 \\
\hline z21 & $z$ & 21 & 2571.379 & 2571.403 & 0.023088 & 8.978838 \\
\hline z22 & $z$ & 22 & 2672.427 & 2672.449 & 0.021642 & 8.098256 \\
\hline
\end{tabular}




\begin{tabular}{|c|c|c|c|c|c|c|}
\hline$x+22$ & $x+$ & 22 & 2715.433 & 2715.457 & 0.024324 & 8.957676 \\
\hline y22 & $y$ & 22 & 2688.446 & 2688.472 & 0.026084 & 9.702259 \\
\hline$z 23$ & $z$ & 23 & 2800.522 & 2800.518 & -0.00402 & -1.43402 \\
\hline y23 & y & 23 & 2816.541 & 2816.566 & 0.025118 & 8.918031 \\
\hline$y-23$ & $y-$ & 23 & 2815.533 & 2815.558 & 0.025131 & 8.925852 \\
\hline$y-24$ & $y-$ & 24 & 2886.57 & 2886.565 & -0.0049 & -1.69716 \\
\hline y24 & $y$ & 24 & 2887.578 & 2887.603 & 0.025043 & 8.672666 \\
\hline z24 & $z$ & 24 & 2871.559 & 2871.586 & 0.026474 & 9.219381 \\
\hline y25 & y & 25 & 2974.61 & 2974.63 & 0.019895 & 6.688272 \\
\hline$x 25$ & $x$ & 25 & 3000.589 & 3000.618 & 0.028274 & 9.422816 \\
\hline$x+26$ & $x+$ & 26 & 3102.645 & 3102.671 & 0.026236 & 8.456001 \\
\hline y26 & $y$ & 26 & 3075.658 & 3075.684 & 0.026462 & 8.603688 \\
\hline$x+27$ & $x+$ & 27 & 3231.687 & 3231.714 & 0.026235 & 8.11804 \\
\hline y27 & $y$ & 27 & 3204.7 & 3204.727 & 0.026597 & 8.299372 \\
\hline$y-27$ & $y-$ & 27 & 3203.692 & 3203.721 & 0.028244 & 8.816087 \\
\hline z28 & $z$ & 28 & 3301.766 & 3301.788 & 0.022385 & 6.779706 \\
\hline y28 & $y$ & 28 & 3317.784 & 3317.811 & 0.026765 & 8.067131 \\
\hline$x+28$ & $x+$ & 28 & 3344.771 & 3344.799 & 0.027638 & 8.263036 \\
\hline$y-29$ & $y^{-}$ & 29 & 3463.845 & 3463.867 & 0.021844 & 6.306296 \\
\hline y29 & $y$ & 29 & 3464.853 & 3464.882 & 0.029082 & 8.39343 \\
\hline$x+29$ & $x+$ & 29 & 3491.84 & 3491.869 & 0.029174 & 8.354899 \\
\hline z29 & $z$ & 29 & 3448.834 & 3448.866 & 0.031977 & 9.271829 \\
\hline$y-30$ & $y-$ & 30 & 3560.898 & 3560.927 & 0.028824 & 8.094597 \\
\hline$y-31$ & $y-$ & 31 & 3673.982 & 3674.01 & 0.028579 & 7.778763 \\
\hline y31 & $y$ & 31 & 3674.99 & 3675.021 & 0.031422 & 8.550228 \\
\hline$x+32$ & $x+$ & 32 & 3758.998 & 3759.024 & 0.025536 & 6.793291 \\
\hline z32 & $z$ & 32 & 3715.992 & 3716.022 & 0.029337 & 7.894796 \\
\hline y32 & y & 32 & 3732.011 & 3732.042 & 0.031202 & 8.36064 \\
\hline y33 & y & 33 & 3895.074 & 3895.11 & 0.035573 & 9.132817 \\
\hline$y-33$ & $y-$ & 33 & 3894.067 & 3894.102 & 0.035756 & 9.182183 \\
\hline$y-34$ & $y-$ & 34 & 3981.099 & 3981.128 & 0.028994 & 7.282922 \\
\hline$x+34$ & $x+$ & 34 & 4009.094 & 4009.13 & 0.036765 & 9.170394 \\
\hline$y-35$ & $y-$ & 35 & 4137.2 & 4137.222 & 0.02217 & 5.358705 \\
\hline$x+35$ & $x+$ & 35 & 4165.195 & 4165.22 & 0.025743 & 6.180496 \\
\hline y35 & $y$ & 35 & 4138.208 & 4138.241 & 0.033628 & 8.126224 \\
\hline z35 & $z$ & 35 & 4122.189 & 4122.224 & 0.035138 & 8.524112 \\
\hline z36 & $z$ & 36 & 4193.226 & 4193.255 & 0.028667 & 6.836503 \\
\hline$x+36$ & $x+$ & 36 & 4236.232 & 4236.266 & 0.03409 & 8.047239 \\
\hline$y-36$ & $y-$ & 36 & 4208.237 & 4208.275 & 0.037705 & 8.959817 \\
\hline$y-37$ & $y-$ & 37 & 4321.321 & 4321.359 & 0.038013 & 8.796623 \\
\hline$x+37$ & $x+$ & 37 & 4349.316 & 4349.354 & 0.038362 & 8.820231 \\
\hline z37 & $z$ & 37 & 4306.31 & 4306.349 & 0.038601 & 8.963823 \\
\hline y37 & y & 37 & 4322.329 & 4322.368 & 0.039367 & 9.107822 \\
\hline
\end{tabular}




\begin{tabular}{|c|c|c|c|c|c|c|}
\hline z38 & $z$ & 38 & 4421.337 & 4421.372 & 0.035587 & 8.048923 \\
\hline$x+38$ & $x+$ & 38 & 4464.343 & 4464.38 & 0.036812 & 8.245776 \\
\hline y38 & $y$ & 38 & 4437.356 & 4437.396 & 0.040382 & 9.100465 \\
\hline$x+39$ & $x+$ & 39 & 4592.401 & 4592.434 & 0.033042 & 7.194922 \\
\hline$y-40$ & $y-$ & 40 & 4635.443 & 4635.475 & 0.031985 & 6.900102 \\
\hline$x+40$ & $x+$ & 40 & 4663.438 & 4663.477 & 0.038656 & 8.289156 \\
\hline y40 & $y$ & 40 & 4636.451 & 4636.494 & 0.042836 & 9.238963 \\
\hline$y-41$ & $y-$ & 41 & 4763.502 & 4763.538 & 0.035708 & 7.496172 \\
\hline z41 & $z$ & 41 & 4748.491 & 4748.534 & 0.04245 & 8.939682 \\
\hline$x+41$ & $x+$ & 41 & 4791.497 & 4791.543 & 0.045872 & 9.573619 \\
\hline$x+42$ & $x+$ & 42 & 4919.592 & 4919.636 & 0.044466 & 9.038548 \\
\hline$x+43$ & $x+$ & 43 & 5020.64 & 5020.64 & 3.40E-05 & 0.006766 \\
\hline y43 & $y$ & 43 & 4993.653 & 4993.689 & 0.036563 & 7.321895 \\
\hline$y-44$ & $y-$ & 44 & 5107.672 & 5107.721 & 0.049308 & 9.65372 \\
\hline$y-45$ & $y-$ & 45 & 5206.74 & 5206.774 & 0.033999 & 6.529812 \\
\hline$y-47$ & $y-$ & 47 & 5463.889 & 5463.935 & 0.046389 & 8.490113 \\
\hline$x+47$ & $x+$ & 47 & 5491.884 & 5491.931 & 0.047079 & 8.572463 \\
\hline$y-48$ & $y-$ & 48 & 5550.921 & 5550.965 & 0.043656 & 7.864647 \\
\hline$x+48$ & $x+$ & 48 & 5578.916 & 5578.962 & 0.046299 & 8.298919 \\
\hline$y-49$ & $y-$ & 49 & 5647.974 & 5648.026 & 0.051983 & 9.203838 \\
\hline$x+50$ & $x+$ & 50 & 5789.053 & 5789.097 & 0.044733 & 7.727166 \\
\hline y50 & $y$ & 50 & 5762.066 & 5762.119 & 0.053796 & 9.336235 \\
\hline$x+51$ & $x+$ & 51 & 5904.08 & 5904.129 & 0.049043 & 8.306624 \\
\hline y51 & $y$ & 51 & 5877.092 & 5877.145 & 0.052922 & 9.004793 \\
\hline$y-51$ & $y-$ & 51 & 5876.085 & 5876.141 & 0.056839 & 9.672943 \\
\hline y52 & $y$ & 52 & 5980.102 & 5980.057 & -0.045 & -7.52562 \\
\hline$x+52$ & $x+$ & 52 & 6007.089 & 6007.064 & -0.0244 & -4.06237 \\
\hline$y-52$ & $y-$ & 52 & 5979.094 & 5979.151 & 0.057557 & 9.62638 \\
\hline$y-53$ & $y-$ & 53 & 6107.189 & 6107.228 & 0.038929 & 6.374296 \\
\hline z54 & $z$ & 54 & 6206.221 & 6206.241 & 0.019831 & 3.195342 \\
\hline$x 54$ & $x$ & 54 & 6248.219 & 6248.26 & 0.041233 & 6.599161 \\
\hline$y-55$ & $y^{-}$ & 55 & 6278.253 & 6278.28 & 0.026982 & 4.297697 \\
\hline$x+55$ & $x+$ & 55 & 6306.248 & 6306.302 & 0.053843 & 8.538035 \\
\hline x56 & $x$ & 56 & 6404.309 & 6404.37 & 0.061315 & 9.574023 \\
\hline$x 58$ & $x$ & 58 & 6616.461 & 6616.52 & 0.059099 & 8.932116 \\
\hline$x+59$ & $x+$ & 59 & 6748.509 & 6748.553 & 0.04345 & 6.438454 \\
\hline z59 & $z$ & 59 & 6705.504 & 6705.565 & 0.061071 & 9.107593 \\
\hline y61 & y & 61 & 6917.644 & 6917.696 & 0.052436 & 7.580038 \\
\hline$x+62$ & $x+$ & 62 & 7059.658 & 7059.667 & 0.009598 & 1.359551 \\
\hline$x+64$ & $x+$ & 64 & 7275.732 & 7275.784 & 0.052284 & 7.186076 \\
\hline y140 & $y$ & 140 & 16179.97 & 16179.85 & -0.12377 & -7.64989 \\
\hline$y-142$ & $y-$ & 142 & 16425.1 & 16425 & -0.09883 & -6.01701 \\
\hline$y-143$ & $y-$ & 143 & 16562.16 & 16562.08 & -0.08206 & -4.9544 \\
\hline
\end{tabular}




$\begin{array}{llrrrrr}x+144 & x+ & 144 & 16704.2 & 16704.08 & -0.11916 & -7.13354 \\ z 169 & z & 169 & 19217.63 & 19217.67 & 0.041989 & 2.184921 \\ x 169 & x & 169 & 19259.62 & 19259.7 & 0.071303 & 3.702201\end{array}$


Table S7B. Summary of UVPD mass spectrum for WT •GDP complex: fragment ions incorporating mass shift of +464.9937 Da (GDP)

\begin{tabular}{|c|c|c|c|c|c|c|}
\hline & $\frac{\text { Ion }}{\text { Tyne }}$ & Residue & Theoretical & Experimental & Amass & $\frac{\mathrm{ppm}}{\text { error }}$ \\
\hline c13 & $\bar{c}$ & 13 & 1786.7 & 1786.687 & -0.01314 & -7.35266 \\
\hline$a+25$ & $a+$ & 25 & 2923.404 & 2923.421 & 0.017064 & 5.837019 \\
\hline c28 & $c$ & 28 & 3346.578 & 3346.608 & 0.030266 & 9.043865 \\
\hline$a+135$ & $a+$ & 135 & 15590.47 & 15590.51 & 0.048484 & 3.109847 \\
\hline a140 & $a$ & 140 & 16165.76 & 16165.9 & 0.135643 & 8.390759 \\
\hline a144 & $\mathrm{a}$ & 144 & 16652.01 & 16652.1 & 0.096146 & 5.773838 \\
\hline a145 & a & 145 & 16753.06 & 16753.1 & 0.041454 & 2.474414 \\
\hline c146 & c & 146 & 16885.11 & 16885.18 & 0.06651 & 3.938974 \\
\hline b161 & $b$ & 161 & 18532.93 & 18533.04 & 0.104193 & 5.622045 \\
\hline a161 & a & 161 & 18504.94 & 18505.09 & 0.152087 & 8.218724 \\
\hline$a+162$ & $a+$ & 162 & 18662.05 & 18662.17 & 0.117615 & 6.302361 \\
\hline$a+164$ & $a+$ & 164 & 18904.18 & 18904.3 & 0.120133 & 6.354838 \\
\hline c165 & c & 165 & 19104.29 & 19104.4 & 0.114557 & 5.996402 \\
\hline$a+166$ & $a+$ & 166 & 19188.37 & 19188.47 & 0.100821 & 5.254274 \\
\hline y45 & y & 45 & 5672.742 & 5672.751 & 0.008988 & 1.584419 \\
\hline$y-69$ & $y-$ & 69 & 8339.117 & 8339.14 & 0.022726 & 2.725232 \\
\hline$x 70$ & $x$ & 70 & 8479.189 & 8479.216 & 0.027824 & 3.281446 \\
\hline$x+71$ & $x+$ & 71 & 8608.255 & 8608.234 & -0.02102 & -2.44173 \\
\hline$y-73$ & $y-$ & 73 & 8865.404 & 8865.403 & -0.00064 & -0.07219 \\
\hline$x+78$ & $x+$ & 78 & 9558.691 & 9558.731 & 0.039932 & 4.177556 \\
\hline$y-82$ & $y^{-}$ & 82 & 10021.93 & 10021.98 & 0.043927 & 4.383089 \\
\hline$x+84$ & $x+$ & 84 & 10265.02 & 10265.04 & 0.019925 & 1.941055 \\
\hline z85 & $z$ & 85 & 10336.06 & 10336.07 & 0.010682 & 1.03347 \\
\hline$x 118$ & $x$ & 118 & 14114.81 & 14114.86 & 0.05277 & 3.738626 \\
\hline z121 & $z$ & 121 & 14405.91 & 14405.99 & 0.072057 & 5.001904 \\
\hline$x+123$ & $x+$ & 123 & 14620.97 & 14621.07 & 0.098257 & 6.720278 \\
\hline$x+127$ & $x+$ & 127 & 15060.25 & 15060.34 & 0.096141 & 6.383758 \\
\hline$y-128$ & $y-$ & 128 & 15160.35 & 15160.38 & 0.033937 & 2.238539 \\
\hline$x+132$ & $x+$ & 132 & 15709.57 & 15709.66 & 0.091735 & 5.839434 \\
\hline$y-133$ & $y-$ & 133 & 15810.61 & 15810.66 & 0.05114 & 3.234538 \\
\hline z133 & z & 133 & 15795.6 & 15795.67 & 0.071846 & 4.548481 \\
\hline y135 & $y$ & 135 & 16025.75 & 16025.76 & 0.006545 & 0.408405 \\
\hline$y-136$ & $y-$ & 136 & 16121.8 & 16121.89 & 0.091297 & 5.662956 \\
\hline z139 & $z$ & 139 & 16513.92 & 16514 & 0.084716 & 5.129975 \\
\hline$x+141$ & $x+$ & 141 & 16771.02 & 16771.11 & 0.088387 & 5.27022 \\
\hline y143 & $y$ & 143 & 17028.16 & 17028.25 & 0.092688 & 5.443218 \\
\hline x149 & $x$ & 149 & 17763.55 & 17763.65 & 0.094512 & 5.320557 \\
\hline
\end{tabular}




$\begin{array}{llrrrrr}z 150 & z & 150 & 17822.6 & 17822.65 & 0.047547 & 2.667792 \\ y 155 & y & 155 & 18294.89 & 18294.92 & 0.02674 & 1.46161 \\ x+157 & x+ & 157 & 18477.97 & 18478.11 & 0.142651 & 7.720057 \\ x 160 & x & 160 & 18662.04 & 18662.17 & 0.126355 & 6.770696 \\ z 164 & z & 164 & 19030.33 & 19030.45 & 0.121227 & 6.370199 \\ y 165 & y & 165 & 19174.44 & 19174.51 & 0.064631 & 3.370684\end{array}$


Table S7C. Summary of UVPD mass spectrum for WT•GDP complex: fragment ions incorporating mass shift of +462.9781 Da (GDP)

\begin{tabular}{|c|c|c|c|c|c|c|}
\hline & $\underline{\text { Ion }}$ & & Theoretical & Experimental & & $\mathrm{ppm}$ \\
\hline Ion & Type & Residue & Mass & Mass & $\Delta$ mass & error \\
\hline a12 & a & 12 & 1682.642 & 1682.632 & -0.00955 & -5.67501 \\
\hline c126 & c & 126 & 14661.99 & 14662.07 & 0.080494 & 5.489977 \\
\hline a131 & a & 131 & 15160.24 & 15160.38 & 0.145542 & 9.600247 \\
\hline c139 & c & 139 & 16095.68 & 16095.83 & 0.151367 & 9.4042 \\
\hline a155 & $a$ & 155 & 17808.55 & 17808.67 & 0.111882 & 6.282486 \\
\hline$a+157$ & $a+$ & 157 & 18027.67 & 18027.8 & 0.127788 & 7.088436 \\
\hline a158 & a & 158 & 18189.72 & 18189.9 & 0.175307 & 9.637694 \\
\hline c160 & c & 160 & 18448.88 & 18449.02 & 0.144813 & 7.849421 \\
\hline c162 & c & 162 & 18704.05 & 18704.16 & 0.110622 & 5.914335 \\
\hline c164 & c & 164 & 18946.17 & 18946.3 & 0.131895 & 6.961564 \\
\hline y15 & y & 15 & 2380.073 & 2380.062 & -0.01086 & -4.5612 \\
\hline$x+20$ & $x+$ & 20 & 2921.262 & 2921.259 & -0.00301 & -1.03039 \\
\hline$y-47$ & $y^{-}$ & 47 & 5926.867 & 5926.894 & 0.027178 & 4.585565 \\
\hline$y-72$ & $y-$ & 72 & 8707.287 & 8707.32 & 0.032574 & 3.741008 \\
\hline$x+75$ & $x+$ & 75 & 9191.505 & 9191.556 & 0.050759 & 5.522378 \\
\hline y75 & $y$ & 75 & 9164.518 & 9164.587 & 0.068855 & 7.513215 \\
\hline y76 & y & 76 & 9301.577 & 9301.636 & 0.058895 & 6.331722 \\
\hline $\mathrm{z} 81$ & $z$ & 81 & 9876.812 & 9876.902 & 0.089743 & 9.086231 \\
\hline z82 & z & 82 & 10004.91 & 10004.91 & 0.006868 & 0.686463 \\
\hline$x+87$ & $x+$ & 87 & 10561.17 & 10561.21 & 0.043371 & 4.106645 \\
\hline$y-90$ & $y^{-}$ & 90 & 10882.32 & 10882.28 & -0.0348 & -3.19757 \\
\hline z122 & $z$ & 122 & 14460.92 & 14461.06 & 0.136886 & 9.465927 \\
\hline$y-122$ & $y-$ & 122 & 14475.93 & 14476.07 & 0.143036 & 9.880956 \\
\hline x124 & $x$ & 124 & 14731.03 & 14731.1 & 0.076684 & 5.205611 \\
\hline z127 & $z$ & 127 & 15015.23 & 15015.31 & 0.088299 & 5.880631 \\
\hline x133 & $x$ & 133 & 15835.58 & 15835.7 & 0.111389 & 7.034095 \\
\hline y136 & $y$ & 136 & 16120.79 & 16120.85 & 0.061183 & 3.795285 \\
\hline$x+136$ & $x+$ & 136 & 16147.78 & 16147.88 & 0.105343 & 6.523682 \\
\hline x136 & $x$ & 136 & 16146.77 & 16146.93 & 0.159846 & 9.899566 \\
\hline$x 138$ & $x$ & 138 & 16424.86 & 16425 & 0.14233 & 8.665523 \\
\hline y141 & $y$ & 141 & 16742.02 & 16742.16 & 0.136976 & 8.18157 \\
\hline x142 & $x$ & 142 & 16915.07 & 16915.17 & 0.104926 & 6.203109 \\
\hline z143 & $z$ & 143 & 17010.13 & 17010.22 & 0.088425 & 5.198374 \\
\hline$x 143$ & $x$ & 143 & 17052.12 & 17052.25 & 0.124629 & 7.308708 \\
\hline z144 & z & 144 & 17124.17 & 17124.29 & 0.11635 & 6.79449 \\
\hline y148 & $y$ & 148 & 17622.47 & 17622.59 & 0.116992 & 6.638796 \\
\hline x148 & $x$ & 148 & 17648.45 & 17648.62 & 0.165851 & 9.397481 \\
\hline$x+151$ & $x+$ & 151 & 17976.68 & 17976.78 & 0.103115 & 5.736042 \\
\hline
\end{tabular}




$\begin{array}{llrrrrr}x+155 & x+ & 155 & 18319.86 & 18320 & 0.137925 & 7.528712 \\ z 156 & z & 156 & 18375.92 & 18376.01 & 0.082295 & 4.478414 \\ x 156 & x & 156 & 18417.92 & 18418.1 & 0.181677 & 9.864142 \\ y 157 & y & 157 & 18448.96 & 18449.02 & 0.056718 & 3.074319 \\ x+158 & x+ & 158 & 18532.97 & 18533.04 & 0.065258 & 3.521182 \\ y-158 & y- & 158 & 18504.98 & 18505.09 & 0.113152 & 6.114681 \\ y-161 & y- & 161 & 18732.11 & 18732.27 & 0.164883 & 8.802162 \\ z 163 & z & 163 & 18915.23 & 18915.32 & 0.091737 & 4.849901 \\ x 163 & x & 163 & 18957.23 & 18957.35 & 0.12276 & 6.47563\end{array}$


Table S7D. Summary of UVPD mass spectrum for WT•GDP complex: fragment ions incorporating mass shift of +463.9859 Da (GDP)

\begin{tabular}{|c|c|c|c|c|c|c|}
\hline & $\underline{\text { Ion }}$ & & Theoretical & Experimental & & $\mathrm{ppm}$ \\
\hline Ion & Type & Residue & Mass & Mass & $\Delta$ mass & error \\
\hline c11 & c & 11 & 1657.633 & 1657.629 & -0.00475 & -2.86553 \\
\hline b12 & b & 12 & 1711.644 & 1711.649 & 0.00507 & 2.962064 \\
\hline 47 & c & 47 & 5642.692 & 5642.74 & 0.04841 & 8.579239 \\
\hline c137 & c & 137 & 15876.6 & 15876.66 & 0.057376 & 3.613871 \\
\hline$a+140$ & $a+$ & 140 & 16165.76 & 16165.9 & 0.135618 & 8.389211 \\
\hline$a+144$ & $a+$ & 144 & 16652.01 & 16652.1 & 0.096121 & 5.772335 \\
\hline$a+145$ & $a+$ & 145 & 16753.06 & 16753.1 & 0.041429 & 2.47292 \\
\hline a157 & a & 157 & 18027.67 & 18027.8 & 0.127813 & 7.089824 \\
\hline b157 & $b$ & 157 & 18055.66 & 18055.8 & 0.138814 & 7.688114 \\
\hline$a+161$ & $a+$ & 161 & 18504.94 & 18505.09 & 0.152062 & 8.217372 \\
\hline$y-15$ & $y-$ & 15 & 2380.073 & 2380.062 & -0.01083 & -4.55069 \\
\hline$x 20$ & $x$ & 20 & 2921.262 & 2921.259 & -0.00299 & -1.02182 \\
\hline $\mathrm{z} 47$ & $z$ & 47 & 5912.864 & 5912.887 & 0.022994 & 3.888809 \\
\hline y69 & $y$ & 69 & 8339.117 & 8339.14 & 0.022701 & 2.722231 \\
\hline$x+70$ & $x+$ & 70 & 8479.189 & 8479.216 & 0.027799 & 3.278494 \\
\hline y73 & $y$ & 73 & 8865.404 & 8865.403 & -0.00066 & -0.07501 \\
\hline$x 75$ & $x$ & 75 & 9191.505 & 9191.556 & 0.050784 & 5.525102 \\
\hline$y-75$ & $y-$ & 75 & 9164.518 & 9164.587 & 0.06888 & 7.515947 \\
\hline z76 & z & 76 & 9286.566 & 9286.598 & 0.031408 & 3.38209 \\
\hline$y-76$ & $y-$ & 76 & 9301.577 & 9301.636 & 0.05892 & 6.334413 \\
\hline$z 77$ & z & 77 & 9399.65 & 9399.655 & 0.004599 & 0.489274 \\
\hline y82 & $y$ & 82 & 10021.93 & 10021.98 & 0.043902 & 4.380592 \\
\hline z83 & $z$ & 83 & 10106.96 & 10106.98 & 0.016818 & 1.664001 \\
\hline$x 87$ & $x$ & 87 & 10561.17 & 10561.21 & 0.043396 & 4.109015 \\
\hline$x+118$ & $x+$ & 118 & 14114.81 & 14114.86 & 0.052745 & 3.736852 \\
\hline y128 & y & 128 & 15160.35 & 15160.38 & 0.033912 & 2.236888 \\
\hline y133 & $y$ & 133 & 15810.61 & 15810.66 & 0.051115 & 3.232955 \\
\hline$y-136$ & $y-$ & 136 & 16120.79 & 16120.85 & 0.061208 & 3.796838 \\
\hline y136 & y & 136 & 16121.8 & 16121.89 & 0.091272 & 5.661403 \\
\hline x136 & $x$ & 136 & 16147.78 & 16147.88 & 0.105368 & 6.525233 \\
\hline$y-141$ & $y-$ & 141 & 16742.02 & 16742.16 & 0.137001 & 8.183066 \\
\hline$y-148$ & $y-$ & 148 & 17622.47 & 17622.59 & 0.117017 & 6.640216 \\
\hline$x+149$ & $x+$ & 149 & 17763.55 & 17763.65 & 0.094487 & 5.319148 \\
\hline x151 & $x$ & 151 & 17976.68 & 17976.78 & 0.10314 & 5.737434 \\
\hline x155 & $x$ & 155 & 18319.86 & 18320 & 0.13795 & 7.530079 \\
\hline$y-157$ & $y-$ & 157 & 18448.96 & 18449.02 & 0.056743 & 3.075676 \\
\hline x158 & $x$ & 158 & 18532.97 & 18533.04 & 0.065283 & 3.522532 \\
\hline$x+160$ & $x+$ & 160 & 18662.04 & 18662.17 & 0.12633 & 6.769355 \\
\hline
\end{tabular}


Table S8A. Summary of UVPD mass spectrum for WT•GDPnP complex: apo fragment ions

Column "Ions" is the fragment ion identity $(a, b, c, x, y, z)$ along with the number of residues in the fragment ion. A plus or minus sign indicates the fragment ion has one additional hydrogen atom or one less hydrogen atom than a conventional fragment ion. Column "Ion Type" is the type of fragment ion $(a, b, c, x, y, z)$. A plus sign indicates a fragment ion $(a, x)$ with an additional hydrogen atom; a minus sign indicates a fragment ion $(y)$ that is missing one hydrogen atom (the addition of one hydrogen atom for $a / x$ ions is not uncommon in UVPD mass spectra of intact proteins); Column "Residue" is the number of residues in the fragment ion. Column "Theoretical Mass" is the theoretical mass of the fragment ion; Column "Experimental Mass" is the experimentally measured mass of the fragment ion. Column "Delta mass" is the mass difference between the theoretical and experimental masses; and column "ppm error" is the mass error in ppm.. The "apo" table (Table S8A) provides the fragment ions that do not retain the ligand. Tables S8B, S8C, and S8D provide the fragment ions that incorporate the ligand based on the mass shift corresponding to the retained ligand (with or without an additional hydrogen atom).

\begin{tabular}{|c|c|c|c|c|c|c|}
\hline Ion & $\frac{\text { Ion }}{\text { Type }}$ & Residue & $\begin{array}{l}\text { Theoretical } \\
\text { Mass }\end{array}$ & $\begin{array}{l}\text { Experimental } \\
\text { Mass }\end{array}$ & $\Delta$ mass & $\begin{array}{l}\text { ppm } \\
\text { error }\end{array}$ \\
\hline$\overline{b 3}$ & $\bar{b}$ & 3 & 289.1096 & 289.1111 & $\overline{0.001441}$ & 4.984268 \\
\hline$a+4$ & $a+$ & 4 & 391.1651 & 391.1671 & 0.001971 & 5.038711 \\
\hline a4 & a & 4 & 390.1573 & 390.1593 & 0.001983 & 5.082565 \\
\hline b4 & $b$ & 4 & 418.1522 & 418.1543 & 0.002124 & 5.07949 \\
\hline c4 & c & 4 & 435.1785 & 435.181 & 0.002466 & 5.66664 \\
\hline a5 & $a$ & 5 & 553.2206 & 553.2237 & 0.003106 & 5.614397 \\
\hline$a+5$ & $a+$ & 5 & 554.2285 & 554.2317 & 0.003216 & 5.802603 \\
\hline b5 & $b$ & 5 & 581.2156 & 581.2188 & 0.003248 & 5.588288 \\
\hline c5 & c & 5 & 598.2419 & 598.2455 & 0.00362 & 6.051064 \\
\hline$a+6$ & $a+$ & 6 & 682.3234 & 682.3274 & 0.003959 & 5.802187 \\
\hline b6 & $b$ & 6 & 709.3105 & 709.3147 & 0.004235 & 5.970587 \\
\hline b7 & $b$ & 7 & 822.3946 & 822.3995 & 0.004892 & 5.948483 \\
\hline$a+7$ & $a+$ & 7 & 795.4075 & 795.4125 & 0.004982 & 6.263416 \\
\hline b8 & $b$ & 8 & 921.463 & 921.4685 & 0.005512 & 5.981792 \\
\hline$a+8$ & $a+$ & 8 & 894.4759 & 894.4815 & 0.005603 & 6.263968 \\
\hline c8 & c & 8 & 938.4893 & 938.495 & 0.005702 & 6.075722 \\
\hline b9 & $b$ & 9 & 1020.531 & 1020.537 & 0.005584 & 5.471659 \\
\hline c9 & c & 9 & 1037.558 & 1037.563 & 0.00559 & 5.387652 \\
\hline$a+9$ & $a+$ & 9 & 993.5443 & 993.5502 & 0.005857 & 5.895024 \\
\hline a9 & $\mathrm{a}$ & 9 & 992.5365 & 992.5423 & 0.00587 & 5.91414 \\
\hline a10 & a & 10 & 1091.605 & 1091.611 & 0.006369 & 5.834529 \\
\hline b10 & $b$ & 10 & 1119.6 & 1119.608 & 0.008036 & 7.177565 \\
\hline$a+11$ & $a+$ & 11 & 1149.634 & 1149.64 & 0.006014 & 5.231201 \\
\hline b11 & $b$ & 11 & 1176.621 & 1176.628 & 0.00673 & 5.719767 \\
\hline c11 & c & 11 & 1193.648 & 1193.655 & 0.007761 & 6.501919 \\
\hline b12 & $b$ & 12 & 1247.658 & 1247.665 & 0.006717 & 5.383685 \\
\hline
\end{tabular}




\begin{tabular}{|c|c|c|c|c|c|c|}
\hline$a+12$ & $a+$ & 12 & 1220.671 & 1220.678 & 0.00699 & 5.726331 \\
\hline c12 & c & 12 & 1264.685 & 1264.692 & 0.007394 & 5.846517 \\
\hline$a+13$ & $a+$ & 13 & 1277.693 & 1277.7 & 0.007137 & 5.585825 \\
\hline c13 & c & 13 & 1321.706 & 1321.714 & 0.007785 & 5.890114 \\
\hline b14 & $b$ & 14 & 1361.701 & 1361.709 & 0.007254 & 5.32716 \\
\hline a14 & a & 14 & 1333.706 & 1333.714 & 0.00754 & 5.653418 \\
\hline$a+14$ & $a+$ & 14 & 1334.714 & 1334.722 & 0.007649 & 5.730791 \\
\hline c14 & c & 14 & 1378.728 & 1378.735 & 0.007687 & 5.575431 \\
\hline$a+15$ & $a+$ & 15 & 1433.783 & 1433.79 & 0.006988 & 4.873799 \\
\hline b15 & $b$ & 15 & 1460.77 & 1460.777 & 0.007447 & 5.097997 \\
\hline c15 & c & 15 & 1477.796 & 1477.804 & 0.008003 & 5.415497 \\
\hline a15 & a & 15 & 1432.775 & 1432.783 & 0.008635 & 6.026767 \\
\hline b16 & $b$ & 16 & 1517.791 & 1517.797 & 0.006007 & 3.957725 \\
\hline c16 & c & 16 & 1534.817 & 1534.827 & 0.009173 & 5.976606 \\
\hline a16 & $a$ & 16 & 1489.796 & 1489.806 & 0.009345 & 6.27267 \\
\hline b17 & $b$ & 17 & 1645.886 & 1645.885 & -0.00118 & -0.71998 \\
\hline a17 & $a$ & 17 & 1617.891 & 1617.899 & 0.008257 & 5.103557 \\
\hline$a+17$ & $a+$ & 17 & 1618.899 & 1618.908 & 0.008518 & 5.261581 \\
\hline c17 & c & 17 & 1662.912 & 1662.921 & 0.00894 & 5.37611 \\
\hline$a+18$ & $a+$ & 18 & 1705.931 & 1705.94 & 0.008732 & 5.118594 \\
\hline c18 & c & 18 & 1749.944 & 1749.954 & 0.009747 & 5.569891 \\
\hline$a+19$ & $a+$ & 19 & 1776.968 & 1776.977 & 0.008854 & 4.982626 \\
\hline a19 & $\mathrm{a}$ & 19 & 1775.96 & 1775.971 & 0.010209 & 5.748439 \\
\hline b19 & $b$ & 19 & 1803.955 & 1803.966 & 0.010289 & 5.703578 \\
\hline c19 & c & 19 & 1820.982 & 1820.992 & 0.010479 & 5.754589 \\
\hline$a+20$ & $a+$ & 20 & 1890.052 & 1890.06 & 0.007876 & 4.167064 \\
\hline c20 & c & 20 & 1934.066 & 1934.077 & 0.011135 & 5.757302 \\
\hline b20 & $b$ & 20 & 1917.039 & 1917.053 & 0.013998 & 7.301885 \\
\hline a21 & $a$ & 21 & 1990.092 & 1990.101 & 0.008426 & 4.233975 \\
\hline$a+21$ & $a+$ & 21 & 1991.1 & 1991.109 & 0.008706 & 4.372442 \\
\hline c21 & $c$ & 21 & 2035.113 & 2035.124 & 0.010574 & 5.19578 \\
\hline b21 & $b$ & 21 & 2018.087 & 2018.099 & 0.011606 & 5.750991 \\
\hline$a+22$ & $a+$ & 22 & 2104.184 & 2104.194 & 0.010217 & 4.855549 \\
\hline c22 & c & 22 & 2148.197 & 2148.209 & 0.011475 & 5.341688 \\
\hline$a+23$ & $a+$ & 23 & 2232.243 & 2232.248 & 0.005789 & 2.593342 \\
\hline b23 & $b$ & 23 & 2259.23 & 2259.242 & 0.01191 & 5.271708 \\
\hline$a+24$ & $a+$ & 24 & 2345.327 & 2345.333 & 0.00669 & 2.852467 \\
\hline b24 & $b$ & 24 & 2372.314 & 2372.327 & 0.012811 & 5.400213 \\
\hline b25 & $b$ & 25 & 2485.398 & 2485.411 & 0.01298 & 5.222504 \\
\hline a25 & $a$ & 25 & 2457.403 & 2457.416 & 0.01351 & 5.497674 \\
\hline $\mathrm{c} 25$ & c & 25 & 2502.424 & 2502.438 & 0.014023 & 5.603766 \\
\hline$a+26$ & $a+$ & 26 & 2586.469 & 2586.48 & 0.010581 & 4.090893 \\
\hline b26 & b & 26 & 2613.456 & 2613.468 & 0.011529 & 4.4114 \\
\hline
\end{tabular}




\begin{tabular}{|c|c|c|c|c|c|c|}
\hline$c 26$ & c & 26 & 2630.483 & 2630.496 & 0.013549 & 5.150766 \\
\hline$a+27$ & $a+$ & 27 & 2700.512 & 2700.524 & 0.011353 & 4.204005 \\
\hline$a+28$ & $a+$ & 28 & 2837.571 & 2837.583 & 0.011917 & 4.199707 \\
\hline b28 & $b$ & 28 & 2864.558 & 2864.573 & 0.014621 & 5.104103 \\
\hline a28 & a & 28 & 2836.563 & 2836.578 & 0.015199 & 5.358245 \\
\hline c28 & c & 28 & 2881.584 & 2881.6 & 0.015469 & 5.368227 \\
\hline$a+29$ & $a+$ & 29 & 2984.64 & 2984.652 & 0.012695 & 4.253434 \\
\hline c29 & c & 29 & 3028.653 & 3028.668 & 0.015418 & 5.090712 \\
\hline b29 & $b$ & 29 & 3011.627 & 3011.644 & 0.017548 & 5.826752 \\
\hline b30 & $b$ & 30 & 3110.695 & 3110.702 & 0.00744 & 2.391748 \\
\hline$a+30$ & $a+$ & 30 & 3083.708 & 3083.721 & 0.012644 & 4.100248 \\
\hline a30 & $\mathrm{a}$ & 30 & 3082.7 & 3082.717 & 0.016488 & 5.348558 \\
\hline$a+31$ & $a+$ & 31 & 3198.735 & 3198.745 & 0.010071 & 3.148422 \\
\hline c31 & c & 31 & 3242.748 & 3242.765 & 0.016504 & 5.08951 \\
\hline a31 & $a$ & 31 & 3197.727 & 3197.745 & 0.018235 & 5.702488 \\
\hline b31 & $b$ & 31 & 3225.722 & 3225.742 & 0.01978 & 6.131961 \\
\hline b32 & $b$ & 32 & 3354.765 & 3354.781 & 0.016327 & 4.86681 \\
\hline c32 & c & 32 & 3371.791 & 3371.81 & 0.019615 & 5.817383 \\
\hline$a+33$ & $a+$ & 33 & 3490.841 & 3490.853 & 0.011999 & 3.437272 \\
\hline b33 & $b$ & 33 & 3517.828 & 3517.85 & 0.02238 & 6.36188 \\
\hline c34 & $c$ & 34 & 3649.881 & 3649.851 & -0.03049 & -8.35452 \\
\hline$a+34$ & $a+$ & 34 & 3605.868 & 3605.882 & 0.013909 & 3.857315 \\
\hline$a+35$ & $a+$ & 35 & 3702.92 & 3702.937 & 0.01644 & 4.43973 \\
\hline c35 & c & 35 & 3746.934 & 3746.951 & 0.017407 & 4.645665 \\
\hline a36 & $a$ & 36 & 3802.96 & 3802.977 & 0.017065 & 4.487294 \\
\hline$a+37$ & $a+$ & 37 & 3917.052 & 3917.064 & 0.012094 & 3.087518 \\
\hline c37 & $c$ & 37 & 3961.066 & 3961.081 & 0.01555 & 3.925711 \\
\hline a37 & a & 37 & 3916.044 & 3916.066 & 0.021798 & 5.566331 \\
\hline a38 & a & 38 & 4045.087 & 4045.105 & 0.018066 & 4.466159 \\
\hline c38 & c & 38 & 4090.108 & 4090.128 & 0.019835 & 4.849505 \\
\hline$a+39$ & $a+$ & 39 & 4161.122 & 4161.135 & 0.013121 & 3.153229 \\
\hline c39 & $c$ & 39 & 4205.135 & 4205.151 & 0.016332 & 3.883823 \\
\hline b39 & b & 39 & 4188.109 & 4188.132 & 0.023589 & 5.632375 \\
\hline$c 40$ & c & 40 & 4292.167 & 4292.136 & -0.03164 & -7.3718 \\
\hline$a+40$ & $a+$ & 40 & 4248.154 & 4248.168 & 0.013806 & 3.249875 \\
\hline c41 & $c$ & 41 & 4455.23 & 4455.195 & -0.03516 & -7.89117 \\
\hline a41 & $a$ & 41 & 4410.209 & 4410.23 & 0.021207 & 4.808615 \\
\hline$a+42$ & $a+$ & 42 & 4567.318 & 4567.338 & 0.019701 & 4.313465 \\
\hline c42 & c & 42 & 4611.332 & 4611.356 & 0.024866 & 5.392369 \\
\hline a42 & a & 42 & 4566.31 & 4566.336 & 0.025255 & 5.530723 \\
\hline$a+43$ & $a+$ & 43 & 4695.413 & 4695.431 & 0.018173 & 3.870366 \\
\hline b43 & $b$ & 43 & 4722.4 & 4722.424 & 0.023693 & 5.017152 \\
\hline$c 43$ & c & 43 & 4739.427 & 4739.453 & 0.026097 & 5.506362 \\
\hline
\end{tabular}




\begin{tabular}{|c|c|c|c|c|c|c|}
\hline$a+44$ & $a+$ & 44 & 4823.472 & 4823.492 & 0.020092 & 4.165458 \\
\hline c44 & $c$ & 44 & 4867.485 & 4867.508 & 0.022693 & 4.662161 \\
\hline$a+45$ & $a+$ & 45 & 4922.54 & 4922.561 & 0.02053 & 4.170604 \\
\hline c45 & c & 45 & 4966.554 & 4966.581 & 0.027892 & 5.615967 \\
\hline a46 & a & 46 & 5020.601 & 5020.619 & 0.018416 & 3.668087 \\
\hline c46 & c & 46 & 5065.622 & 5065.647 & 0.025198 & 4.974315 \\
\hline$a+47$ & $a+$ & 47 & 5134.693 & 5134.707 & 0.014592 & 2.841839 \\
\hline c47 & c & 47 & 5178.706 & 5178.744 & 0.038263 & 7.388525 \\
\hline$a+48$ & $a+$ & 48 & 5249.72 & 5249.743 & 0.023349 & 4.44766 \\
\hline b48 & b & 48 & 5276.707 & 5276.744 & 0.037671 & 7.139112 \\
\hline$a+49$ & $a+$ & 49 & 5306.741 & 5306.758 & 0.017414 & 3.281481 \\
\hline$a+50$ & $a+$ & 50 & 5435.784 & 5435.805 & 0.020966 & 3.857028 \\
\hline c51 & c & 51 & 5580.845 & 5580.864 & 0.019711 & 3.531903 \\
\hline$a+51$ & $a+$ & 51 & 5536.831 & 5536.852 & 0.020405 & 3.685315 \\
\hline b51 & b & 51 & 5563.818 & 5563.854 & 0.035513 & 6.382847 \\
\hline a52 & $a$ & 52 & 5638.833 & 5638.852 & 0.019406 & 3.441492 \\
\hline b52 & $b$ & 52 & 5666.828 & 5666.86 & 0.03267 & 5.76513 \\
\hline a53 & $a$ & 53 & 5751.917 & 5751.938 & 0.021284 & 3.700332 \\
\hline a54 & a & 54 & 5865.001 & 5865.028 & 0.026824 & 4.573571 \\
\hline$a+55$ & $a+$ & 55 & 5981.036 & 5981.059 & 0.023431 & 3.917544 \\
\hline$a+56$ & $a+$ & 56 & 6094.12 & 6094.131 & 0.011392 & 1.869338 \\
\hline b56 & $b$ & 56 & 6121.107 & 6121.138 & 0.030937 & 5.054151 \\
\hline$c 57$ & c & 57 & 6251.217 & 6251.196 & -0.02156 & -3.44845 \\
\hline a57 & $a$ & 57 & 6206.196 & 6206.216 & 0.020606 & 3.32023 \\
\hline$a+58$ & $a+$ & 58 & 6322.231 & 6322.244 & 0.013185 & 2.085493 \\
\hline c58 & c & 58 & 6366.244 & 6366.271 & 0.027139 & 4.262953 \\
\hline c59 & c & 59 & 6467.292 & 6467.286 & -0.00598 & -0.92465 \\
\hline$a+59$ & $a+$ & 59 & 6423.278 & 6423.295 & 0.016286 & 2.53546 \\
\hline a60 & a & 60 & 6493.308 & 6493.309 & 0.001406 & 0.216531 \\
\hline b60 & $b$ & 60 & 6521.302 & 6521.333 & 0.030416 & 4.664099 \\
\hline$a+61$ & $a+$ & 61 & 6551.337 & 6551.353 & 0.015822 & 2.415075 \\
\hline c61 & $\mathrm{C}$ & 61 & 6595.35 & 6595.376 & 0.025607 & 3.882584 \\
\hline$a+62$ & $a+$ & 62 & 6679.395 & 6679.423 & 0.027428 & 4.106355 \\
\hline a62 & a & 62 & 6678.388 & 6678.418 & 0.030537 & 4.572511 \\
\hline a63 & a & 63 & 6807.43 & 6807.46 & 0.030274 & 4.447199 \\
\hline a64 & a & 64 & 6936.473 & 6936.505 & 0.032437 & 4.676296 \\
\hline a65 & a & 65 & 7099.536 & 7099.572 & 0.035606 & 5.015257 \\
\hline$a+66$ & $a+$ & 66 & 7187.576 & 7187.542 & -0.0338 & -4.70215 \\
\hline a69 & $\mathrm{a}$ & 69 & 7544.747 & 7544.779 & 0.031646 & 4.194442 \\
\hline$a+70$ & $a+$ & 70 & 7660.782 & 7660.81 & 0.028131 & 3.672075 \\
\hline$a+71$ & $a+$ & 71 & 7788.84 & 7788.867 & 0.027168 & 3.488063 \\
\hline a72 & a & 72 & 7950.896 & 7950.926 & 0.030745 & 3.86686 \\
\hline a73 & a & 73 & 8081.936 & 8081.964 & 0.027853 & 3.446328 \\
\hline
\end{tabular}




\begin{tabular}{|c|c|c|c|c|c|c|}
\hline$a+74$ & $a+$ & 74 & 8239.045 & 8239.087 & 0.041402 & 5.025093 \\
\hline a75 & $a$ & 75 & 8339.085 & 8339.114 & 0.029012 & 3.479039 \\
\hline a76 & $a$ & 76 & 8396.106 & 8396.14 & 0.03392 & 4.039968 \\
\hline a77 & $a$ & 77 & 8525.149 & 8525.186 & 0.0371 & 4.35183 \\
\hline$a+78$ & $a+$ & 78 & 8583.178 & 8583.211 & 0.032357 & 3.769812 \\
\hline b78 & $b$ & 78 & 8610.165 & 8610.215 & 0.049662 & 5.767833 \\
\hline b79 & $b$ & 79 & 8757.234 & 8757.273 & 0.039078 & 4.462368 \\
\hline a79 & a & 79 & 8729.239 & 8729.28 & 0.041383 & 4.740734 \\
\hline a80 & $a$ & 80 & 8842.323 & 8842.359 & 0.036272 & 4.102089 \\
\hline c81 & c & 81 & 8990.353 & 8990.396 & 0.042366 & 4.712384 \\
\hline c82 & c & 82 & 9089.422 & 9089.448 & 0.02669 & 2.93638 \\
\hline a82 & a & 82 & 9044.401 & 9044.466 & 0.065605 & 7.253659 \\
\hline a83 & $a$ & 83 & 9191.469 & 9191.517 & 0.047977 & 5.219731 \\
\hline a84 & a & 84 & 9262.506 & 9262.52 & 0.014285 & 1.542239 \\
\hline a85 & $a$ & 85 & 9375.59 & 9375.622 & 0.031421 & 3.351362 \\
\hline a86 & a & 86 & 9489.633 & 9489.677 & 0.043928 & 4.629051 \\
\hline a87 & $a$ & 87 & 9603.676 & 9603.743 & 0.067378 & 7.015855 \\
\hline a88 & a & 88 & 9704.724 & 9704.799 & 0.075737 & 7.804138 \\
\hline$a+89$ & $a+$ & 89 & 9833.826 & 9833.853 & 0.026297 & 2.674134 \\
\hline$a+90$ & $a+$ & 90 & 9920.859 & 9920.895 & 0.036503 & 3.679416 \\
\hline a91 & $a$ & 91 & 10066.92 & 10066.96 & 0.040371 & 4.010264 \\
\hline a92 & a & 92 & 10195.96 & 10196.02 & 0.05784 & 5.672834 \\
\hline$a+93$ & $a+$ & 93 & 10312 & 10312.04 & 0.046024 & 4.463148 \\
\hline a94 & $\mathrm{a}$ & 94 & 10424.07 & 10424.12 & 0.049328 & 4.732124 \\
\hline a95 & $a$ & 95 & 10561.13 & 10561.17 & 0.040589 & 3.843243 \\
\hline$a+96$ & $a+$ & 96 & 10699.2 & 10699.24 & 0.042631 & 3.984501 \\
\hline a97 & $a$ & 97 & 10861.25 & 10861.29 & 0.037489 & 3.451627 \\
\hline a131 & a & 131 & 14697.26 & 14697.11 & -0.14418 & -9.80999 \\
\hline a169 & $a$ & 169 & 19116.57 & 19116.44 & -0.12436 & -6.50525 \\
\hline$y-3$ & $y-$ & 3 & 402.2352 & 402.2372 & 0.00198 & 4.922572 \\
\hline z3 & $z$ & 3 & 387.2243 & 387.2263 & 0.001995 & 5.152053 \\
\hline y3 & $y$ & 3 & 403.2431 & 403.2451 & 0.002029 & 5.031705 \\
\hline$x 3$ & $x$ & 3 & 429.2223 & 429.2245 & 0.002164 & 5.041676 \\
\hline y4 & $y$ & 4 & 540.302 & 540.305 & 0.002994 & 5.541346 \\
\hline$x 4$ & $x$ & 4 & 566.2813 & 566.2844 & 0.00316 & 5.580266 \\
\hline z5 & $z$ & 5 & 652.3782 & 652.3821 & 0.003887 & 5.958201 \\
\hline y5 & y & 5 & 668.3969 & 668.4009 & 0.00392 & 5.864778 \\
\hline$x 5$ & $x$ & 5 & 694.3762 & 694.3803 & 0.004087 & 5.885858 \\
\hline$x 6$ & $x$ & 6 & 850.4773 & 850.4823 & 0.004966 & 5.839074 \\
\hline y6 & y & 6 & 824.4981 & 824.503 & 0.004983 & 6.043677 \\
\hline z6 & $z$ & 6 & 808.4793 & 808.4844 & 0.005072 & 6.273506 \\
\hline y7 & $y$ & 7 & 937.5821 & 937.5877 & 0.005579 & 5.950412 \\
\hline$x 7$ & $x$ & 7 & 963.5614 & 963.567 & 0.005623 & 5.835643 \\
\hline
\end{tabular}




\begin{tabular}{|c|c|c|c|c|c|c|}
\hline z7 & z & 7 & 921.5634 & 921.5691 & 0.005728 & 6.215525 \\
\hline y8 & $y$ & 8 & 1066.625 & 1066.631 & 0.006568 & 6.157742 \\
\hline z9 & $z$ & 9 & 1206.707 & 1206.714 & 0.006743 & 5.587935 \\
\hline$x 9$ & $x$ & 9 & 1248.705 & 1248.712 & 0.007004 & 5.609011 \\
\hline y9 & y & 9 & 1222.726 & 1222.733 & 0.007021 & 5.742089 \\
\hline$x 10$ & $x$ & 10 & 1347.773 & 1347.781 & 0.007686 & 5.702739 \\
\hline y10 & y & 10 & 1321.794 & 1321.802 & 0.007825 & 5.919983 \\
\hline$x 11$ & $x$ & 11 & 1460.858 & 1460.866 & 0.008111 & 5.552218 \\
\hline y11 & y & 11 & 1434.878 & 1434.887 & 0.008481 & 5.910606 \\
\hline$x 12$ & $x$ & 12 & 1561.905 & 1561.913 & 0.008148 & 5.216706 \\
\hline y12 & $y$ & 12 & 1535.926 & 1535.935 & 0.008598 & 5.597926 \\
\hline$y-13$ & $y^{-}$ & 13 & 1697.981 & 1697.987 & 0.00593 & 3.492401 \\
\hline z13 & $z$ & 13 & 1682.971 & 1682.98 & 0.009057 & 5.381556 \\
\hline$x 13$ & $x$ & 13 & 1724.969 & 1724.979 & 0.010417 & 6.038951 \\
\hline y13 & y & 13 & 1698.989 & 1699 & 0.010678 & 6.284913 \\
\hline$y-14$ & $y^{-}$ & 14 & 1845.05 & 1845.056 & 0.006368 & 3.451415 \\
\hline z14 & $z$ & 14 & 1830.039 & 1830.049 & 0.009739 & 5.321745 \\
\hline$x 14$ & $x$ & 14 & 1872.037 & 1872.048 & 0.010855 & 5.798497 \\
\hline y14 & $y$ & 14 & 1846.058 & 1846.069 & 0.011482 & 6.219741 \\
\hline$x+15$ & $x+$ & 15 & 1944.082 & 1944.088 & 0.006081 & 3.127938 \\
\hline z15 & $z$ & 15 & 1901.076 & 1901.086 & 0.010105 & 5.315411 \\
\hline y15 & y & 15 & 1917.095 & 1917.106 & 0.011603 & 6.052387 \\
\hline$x+16$ & $x+$ & 16 & 2059.109 & 2059.116 & 0.006729 & 3.267903 \\
\hline y16 & $y$ & 16 & 2032.122 & 2032.131 & 0.009228 & 4.541066 \\
\hline y17 & $y$ & 17 & 2147.149 & 2147.16 & 0.011556 & 5.382021 \\
\hline y18 & y & 18 & 2246.217 & 2246.229 & 0.01175 & 5.231017 \\
\hline$x 18$ & $x$ & 18 & 2272.196 & 2272.209 & 0.012954 & 5.701092 \\
\hline$y-19$ & $y-$ & 19 & 2302.231 & 2302.241 & 0.010078 & 4.377507 \\
\hline y19 & $y$ & 19 & 2303.239 & 2303.249 & 0.010798 & 4.688181 \\
\hline y20 & y & 20 & 2431.297 & 2431.309 & 0.012276 & 5.049157 \\
\hline$x+21$ & $x+$ & 21 & 2614.385 & 2614.395 & 0.010061 & 3.848311 \\
\hline z21 & $z$ & 21 & 2571.379 & 2571.391 & 0.011583 & 4.504586 \\
\hline y21 & y & 21 & 2587.398 & 2587.412 & 0.013461 & 5.202523 \\
\hline y22 & y & 22 & 2688.446 & 2688.461 & 0.015342 & 5.706643 \\
\hline$x+23$ & $x+$ & 23 & 2843.528 & 2843.539 & 0.011098 & 3.902887 \\
\hline$y-23$ & $y-$ & 23 & 2815.533 & 2815.546 & 0.012924 & 4.590261 \\
\hline z23 & $z$ & 23 & 2800.522 & 2800.536 & 0.014085 & 5.029419 \\
\hline y23 & y & 23 & 2816.541 & 2816.555 & 0.014132 & 5.017502 \\
\hline y24 & y & 24 & 2887.578 & 2887.57 & -0.00764 & -2.64755 \\
\hline z24 & z & 24 & 2871.559 & 2871.574 & 0.014938 & 5.202052 \\
\hline$y-24$ & $y^{-}$ & 24 & 2886.57 & 2886.585 & 0.015168 & 5.25469 \\
\hline$x+24$ & $x+$ & 24 & 2914.565 & 2914.587 & 0.021867 & 7.502652 \\
\hline$x+25$ & $x+$ & 25 & 3001.597 & 3001.611 & 0.013797 & 4.596542 \\
\hline
\end{tabular}




\begin{tabular}{|c|c|c|c|c|c|c|}
\hline z25 & z & 25 & 2958.591 & 2958.605 & 0.013853 & 4.682296 \\
\hline y25 & y & 25 & 2974.61 & 2974.624 & 0.014253 & 4.791552 \\
\hline$x 25$ & $x$ & 25 & 3000.589 & 3000.607 & 0.017496 & 5.830855 \\
\hline$y-26$ & $y-$ & 26 & 3074.65 & 3074.663 & 0.013339 & 4.338391 \\
\hline y26 & $y$ & 26 & 3075.658 & 3075.672 & 0.014743 & 4.793446 \\
\hline$x 26$ & $x$ & 26 & 3101.637 & 3101.654 & 0.01746 & 5.629285 \\
\hline z27 & $z$ & 27 & 3188.682 & 3188.695 & 0.013925 & 4.367009 \\
\hline$x+27$ & $x+$ & 27 & 3231.687 & 3231.701 & 0.014098 & 4.362417 \\
\hline y27 & $y$ & 27 & 3204.7 & 3204.716 & 0.015898 & 4.960838 \\
\hline y28 & $y$ & 28 & 3317.784 & 3317.796 & 0.011337 & 3.417039 \\
\hline z28 & z & 28 & 3301.766 & 3301.777 & 0.011764 & 3.562942 \\
\hline y29 & y & 29 & 3464.853 & 3464.868 & 0.015532 & 4.48273 \\
\hline$x+29$ & $x+$ & 29 & 3491.84 & 3491.855 & 0.015624 & 4.474423 \\
\hline$y-29$ & $y-$ & 29 & 3463.845 & 3463.864 & 0.019159 & 5.531146 \\
\hline z29 & $z$ & 29 & 3448.834 & 3448.854 & 0.019893 & 5.768036 \\
\hline$x 29$ & $x$ & 29 & 3490.832 & 3490.853 & 0.020729 & 5.938126 \\
\hline$y-30$ & $y^{-}$ & 30 & 3560.898 & 3560.913 & 0.015275 & 4.289658 \\
\hline$x+30$ & $x+$ & 30 & 3588.893 & 3588.917 & 0.024144 & 6.727414 \\
\hline$y-31$ & $y^{-}$ & 31 & 3673.982 & 3673.992 & 0.010513 & 2.861482 \\
\hline$x+31$ & $x+$ & 31 & 3701.977 & 3701.992 & 0.015767 & 4.259067 \\
\hline y31 & y & 31 & 3674.99 & 3675.009 & 0.018984 & 5.165729 \\
\hline x31 & $x$ & 31 & 3700.969 & 3700.998 & 0.029282 & 7.911982 \\
\hline$x+32$ & $x+$ & 32 & 3758.998 & 3759.004 & 0.005368 & 1.428032 \\
\hline z32 & $z$ & 32 & 3715.992 & 3715.999 & 0.007052 & 1.897743 \\
\hline y32 & y & 32 & 3732.011 & 3732.028 & 0.016554 & 4.435678 \\
\hline y33 & y & 33 & 3895.074 & 3895.096 & 0.021413 & 5.497456 \\
\hline z34 & $z$ & 34 & 3966.088 & 3966.105 & 0.016863 & 4.251797 \\
\hline$x+34$ & $x+$ & 34 & 4009.094 & 4009.112 & 0.018454 & 4.603028 \\
\hline y34 & y & 34 & 3982.106 & 3982.127 & 0.020559 & 5.162845 \\
\hline$x+35$ & $x+$ & 35 & 4165.195 & 4165.205 & 0.010742 & 2.578983 \\
\hline y35 & y & 35 & 4138.208 & 4138.224 & 0.016298 & 3.93842 \\
\hline z35 & $z$ & 35 & 4122.189 & 4122.208 & 0.019024 & 4.615024 \\
\hline$y-35$ & $y^{-}$ & 35 & 4137.2 & 4137.221 & 0.020902 & 5.052217 \\
\hline z36 & $z$ & 36 & 4193.226 & 4193.239 & 0.013286 & 3.168444 \\
\hline$x+36$ & $x+$ & 36 & 4236.232 & 4236.25 & 0.018174 & 4.290126 \\
\hline$y-36$ & $y^{-}$ & 36 & 4208.237 & 4208.256 & 0.019596 & 4.65659 \\
\hline y36 & $y$ & 36 & 4209.245 & 4209.265 & 0.020204 & 4.799911 \\
\hline$x+37$ & $x+$ & 37 & 4349.316 & 4349.335 & 0.018952 & 4.35746 \\
\hline$y-37$ & $y-$ & 37 & 4321.321 & 4321.342 & 0.021456 & 4.965156 \\
\hline$x+38$ & $x+$ & 38 & 4464.343 & 4464.362 & 0.0196 & 4.390337 \\
\hline y38 & y & 38 & 4437.356 & 4437.378 & 0.022071 & 4.973908 \\
\hline z38 & z & 38 & 4421.337 & 4421.362 & 0.025129 & 5.683575 \\
\hline$x+39$ & $x+$ & 39 & 4592.401 & 4592.418 & 0.016196 & 3.526688 \\
\hline
\end{tabular}




\begin{tabular}{|c|c|c|c|c|c|c|}
\hline$y-39$ & $y-$ & 39 & 4564.406 & 4564.436 & 0.029835 & 6.536454 \\
\hline$y-40$ & $y-$ & 40 & 4635.443 & 4635.464 & 0.020334 & 4.386642 \\
\hline$x+40$ & $x+$ & 40 & 4663.438 & 4663.459 & 0.021078 & 4.519834 \\
\hline y40 & $y$ & 40 & 4636.451 & 4636.473 & 0.021522 & 4.641912 \\
\hline$x 40$ & $x$ & 40 & 4662.431 & 4662.456 & 0.025485 & 5.466033 \\
\hline z40 & $z$ & 40 & 4620.433 & 4620.459 & 0.026275 & 5.686697 \\
\hline$y-41$ & $y-$ & 41 & 4763.502 & 4763.519 & 0.017153 & 3.600929 \\
\hline z41 & $z$ & 41 & 4748.491 & 4748.515 & 0.023773 & 5.006432 \\
\hline$x+41$ & $x+$ & 41 & 4791.497 & 4791.522 & 0.025365 & 5.293746 \\
\hline x42 & $x$ & 42 & 4918.584 & 4918.609 & 0.024411 & 4.963014 \\
\hline y42 & y & 42 & 4892.605 & 4892.631 & 0.025696 & 5.252008 \\
\hline$x+42$ & $x+$ & 42 & 4919.592 & 4919.619 & 0.027376 & 5.564683 \\
\hline$y-42$ & $y-$ & 42 & 4891.597 & 4891.628 & 0.031364 & 6.411818 \\
\hline$x+43$ & $x+$ & 43 & 5020.64 & 5020.619 & -0.02047 & -4.07797 \\
\hline z43 & $z$ & 43 & 4977.634 & 4977.654 & 0.020415 & 4.101346 \\
\hline y43 & y & 43 & 4993.653 & 4993.678 & 0.025868 & 5.180176 \\
\hline$x 43$ & $x$ & 43 & 5019.632 & 5019.666 & 0.034094 & 6.792132 \\
\hline$y-44$ & $y^{-}$ & 44 & 5107.672 & 5107.69 & 0.018546 & 3.631015 \\
\hline$x 44$ & $x$ & 44 & 5134.659 & 5134.707 & 0.048472 & 9.44016 \\
\hline$y-45$ & $y-$ & 45 & 5206.74 & 5206.76 & 0.020374 & 3.913011 \\
\hline$x+45$ & $x+$ & 45 & 5234.735 & 5234.774 & 0.039206 & 7.48958 \\
\hline$y-47$ & $y-$ & 47 & 5463.889 & 5463.913 & 0.024416 & 4.468618 \\
\hline$x+48$ & $x+$ & 48 & 5578.916 & 5578.937 & 0.020909 & 3.747855 \\
\hline z48 & $z$ & 48 & 5535.91 & 5535.932 & 0.022369 & 4.040709 \\
\hline y48 & y & 48 & 5551.929 & 5551.953 & 0.0246 & 4.430893 \\
\hline y49 & $\mathrm{y}$ & 49 & 5648.981 & 5649.007 & 0.025461 & 4.507184 \\
\hline$x+50$ & $x+$ & 50 & 5789.053 & 5789.078 & 0.02569 & 4.437681 \\
\hline y50 & $y$ & 50 & 5762.066 & 5762.098 & 0.032312 & 5.607711 \\
\hline$x+51$ & $x+$ & 51 & 5904.08 & 5904.106 & 0.026094 & 4.41965 \\
\hline y51 & $y$ & 51 & 5877.092 & 5877.122 & 0.029484 & 5.016766 \\
\hline$y-51$ & $y^{-}$ & 51 & 5876.085 & 5876.119 & 0.034052 & 5.795021 \\
\hline$x+52$ & $x+$ & 52 & 6007.089 & 6007.052 & -0.03722 & -6.19618 \\
\hline$y-52$ & $y^{-}$ & 52 & 5979.094 & 5979.127 & 0.033283 & 5.566568 \\
\hline y53 & $y$ & 53 & 6108.197 & 6108.228 & 0.031104 & 5.092174 \\
\hline$x+53$ & $x+$ & 53 & 6135.184 & 6135.218 & 0.033879 & 5.522079 \\
\hline x54 & $x$ & 54 & 6248.219 & 6248.185 & -0.03357 & -5.37289 \\
\hline z54 & $z$ & 54 & 6206.221 & 6206.216 & -0.00434 & -0.69914 \\
\hline$y-54$ & $y-$ & 54 & 6221.232 & 6221.276 & 0.044339 & 7.12705 \\
\hline$y-55$ & $y^{-}$ & 55 & 6278.253 & 6278.261 & 0.007743 & 1.23331 \\
\hline x56 & $x$ & 56 & 6404.309 & 6404.343 & 0.034378 & 5.367949 \\
\hline$x+57$ & $x+$ & 57 & 6518.401 & 6518.434 & 0.033209 & 5.09465 \\
\hline x58 & $x$ & 58 & 6616.461 & 6616.497 & 0.035871 & 5.421478 \\
\hline$x+58$ & $x+$ & 58 & 6617.469 & 6617.507 & 0.037643 & 5.688424 \\
\hline
\end{tabular}




$\begin{array}{llrrrrr}x+59 & x+ & 59 & 6748.509 & 6748.529 & 0.019036 & 2.820766 \\ y-59 & y- & 59 & 6720.515 & 6720.544 & 0.029087 & 4.328096 \\ z 59 & z & 59 & 6705.504 & 6705.539 & 0.035054 & 5.227646 \\ z 61 & z & 61 & 6901.625 & 6901.65 & 0.025436 & 3.685509 \\ x+61 & x+ & 61 & 6944.631 & 6944.666 & 0.035561 & 5.120642 \\ y-61 & y- & 61 & 6916.636 & 6916.675 & 0.039765 & 5.749187 \\ y 62 & y & 62 & 7032.67 & 7032.699 & 0.0282 & 4.009857 \\ x+63 & x+ & 63 & 7188.7 & 7188.725 & 0.024625 & 3.425511 \\ x+64 & x+ & 64 & 7275.732 & 7275.759 & 0.026298 & 3.614477 \\ x+65 & x+ & 65 & 7390.759 & 7390.816 & 0.056475 & 7.641295 \\ y-68 & y- & 68 & 7746.029 & 7746.068 & 0.039094 & 5.046977 \\ x 133 & x & 133 & 15372.61 & 15372.53 & -0.07746 & -5.03857 \\ y-144 & y- & 144 & 16676.2 & 16676.08 & -0.11913 & -7.14353 \\ \text { z169 } & z & 169 & 19217.63 & 19217.48 & -0.14636 & -7.61572 \\ x+169 & x+ & 169 & 19260.63 & 19260.51 & -0.12156 & -6.31142 \\ y 169 & y & 169 & 19233.65 & 19233.54 & -0.10178 & -5.29156\end{array}$


Table S8B. Summary of UVPD mass spectrum for WT•GDPnP complex: fragment ions incorporating mass shift of 543.9760 Da (GDPnP)

\begin{tabular}{|c|c|c|c|c|c|c|}
\hline & $\underline{\text { Ion }}$ & & Theoretical & Experimental & & $\mathrm{ppm}$ \\
\hline Ion & Type & $\underline{\text { Residue }}$ & $\underline{\text { Mass }}$ & Mass & $\underline{\Delta \text { mass }}$ & error \\
\hline a1 & a & 1 & 573.0025 & 573 & -0.00255 & -4.44152 \\
\hline b1 & $b$ & 1 & 600.9975 & 601 & 0.00254 & 4.226307 \\
\hline a10 & a & 10 & 1635.581 & 1635.581 & $9.50 \mathrm{E}-05$ & 0.058083 \\
\hline a15 & a & 15 & 1976.751 & 1976.732 & -0.01856 & -9.38915 \\
\hline c136 & c & 136 & 15869.56 & 15869.61 & 0.047143 & 2.970655 \\
\hline$a+149$ & $a+$ & 149 & 17220.26 & 17220.21 & -0.04455 & -2.58719 \\
\hline a150 & $a$ & 150 & 17375.35 & 17375.34 & -0.01528 & -0.87935 \\
\hline b158 & $b$ & 158 & 18298.72 & 18298.85 & 0.134787 & 7.365926 \\
\hline$a+159$ & $a+$ & 159 & 18372.78 & 18372.91 & 0.134744 & 7.333892 \\
\hline a160 & a & 160 & 18484.85 & 18484.99 & 0.14054 & 7.602982 \\
\hline a162 & a & 162 & 18740.02 & 18740.08 & 0.052319 & 2.791832 \\
\hline b162 & $b$ & 162 & 18768.02 & 18768.08 & 0.065949 & 3.513903 \\
\hline c162 & c & 162 & 18785.04 & 18785.14 & 0.093604 & 4.9829 \\
\hline a163 & a & 163 & 18869.07 & 18869.15 & 0.084586 & 4.482787 \\
\hline b163 & b & 163 & 18897.06 & 18897.19 & 0.127723 & 6.758882 \\
\hline c164 & c & 164 & 19027.17 & 19027.27 & 0.097174 & 5.107118 \\
\hline z16 & $z$ & 16 & 2560.079 & 2560.086 & 0.006751 & 2.637028 \\
\hline z46 & $z$ & 46 & 5836.753 & 5836.793 & 0.039861 & 6.829311 \\
\hline y46 & y & 46 & 5852.772 & 5852.819 & 0.047856 & 8.176639 \\
\hline$x+73$ & $x+$ & 73 & 8972.381 & 8972.366 & -0.01517 & -1.69041 \\
\hline y77 & y & 77 & 9495.659 & 9495.68 & 0.021248 & 2.237654 \\
\hline$x 77$ & $x$ & 77 & 9521.638 & 9521.698 & 0.060122 & 6.31425 \\
\hline z79 & z & 79 & 9723.71 & 9723.79 & 0.080101 & 8.2377 \\
\hline z80 & $z$ & 80 & 9870.778 & 9870.862 & 0.083454 & 8.454653 \\
\hline$x+87$ & $x+$ & 87 & 10642.17 & 10642.25 & 0.082415 & 7.744191 \\
\hline y89 & y & 89 & 10861.32 & 10861.29 & -0.02418 & -2.22634 \\
\hline y119 & y & 119 & 14270.82 & 14270.84 & 0.016886 & 1.183253 \\
\hline$y-120$ & $y-$ & 120 & 14370.86 & 14370.9 & 0.036831 & 2.562896 \\
\hline y121 & $y$ & 121 & 14500.91 & 14501.02 & 0.109943 & 7.581798 \\
\hline$y-123$ & $y-$ & 123 & 14671.96 & 14672 & 0.044045 & 3.001988 \\
\hline y124 & y & 124 & 14786.05 & 14786.07 & 0.01994 & 1.348569 \\
\hline$x 126$ & $x$ & 126 & 15010.16 & 15010.23 & 0.065499 & 4.363643 \\
\hline y128 & $y$ & 128 & 15240.34 & 15240.29 & -0.04286 & -2.81214 \\
\hline$x 128$ & $x$ & 128 & 15266.32 & 15266.34 & 0.019137 & 1.253544 \\
\hline z131 & $z$ & 131 & 15630.51 & 15630.51 & -0.00376 & -0.24036 \\
\hline$y-132$ & $y-$ & 132 & 15760.55 & 15760.57 & 0.021238 & 1.347544 \\
\hline y133 & y & 133 & 15890.6 & 15890.63 & 0.024394 & 1.535121 \\
\hline
\end{tabular}




$\begin{array}{llrrrrr}\text { x+134 } & x+ & 134 & 16030.67 & 16030.7 & 0.028928 & 1.804538 \\ \text { x135 } & x & 135 & 16130.71 & 16130.78 & 0.063536 & 3.938821 \\ \text { y-136 } & y- & 136 & 16200.78 & 16200.79 & 0.013735 & 0.847801 \\ \text { y-137 } & y- & 137 & 16315.81 & 16315.89 & 0.081351 & 4.986026 \\ \text { x138 } & x & 138 & 16505.86 & 16505.93 & 0.068211 & 4.132533 \\ \text { y139 } & y & 139 & 16608.92 & 16608.88 & -0.03706 & -2.23127 \\ \text { z141 } & z & 141 & 16807 & 16807.02 & 0.027049 & 1.609389 \\ \text { x141 } & x & 141 & 16849 & 16849.04 & 0.04067 & 2.413794 \\ \text { y-142 } & y- & 142 & 16969.08 & 16969.07 & -0.01135 & -0.66904 \\ \text { y-144 } & y- & 144 & 17220.18 & 17220.21 & 0.034268 & 1.989993 \\ \text { x145 } & x & 145 & 17375.22 & 17375.34 & 0.111156 & 6.397385 \\ \text { y148 } & y & 148 & 17703.47 & 17703.47 & -0.00464 & -0.26204 \\ \text { x148 } & x & 148 & 17729.45 & 17729.5 & 0.052913 & 2.984469 \\ \text { z149 } & z & 149 & 17800.54 & 17800.54 & 0.005505 & 0.30926 \\ \text { y-149 } & y- & 149 & 17815.55 & 17815.57 & 0.018247 & 1.02422 \\ \text { x149 } & x & 149 & 17842.53 & 17842.58 & 0.043364 & 2.430372 \\ \text { z151 } & z & 151 & 18014.67 & 18014.68 & 0.012925 & 0.717471 \\ \text { y152 } & y & 152 & 18101.72 & 18101.75 & 0.022432 & 1.239219 \\ \text { y154 } & y & 154 & 18316.85 & 18316.89 & 0.043439 & 2.371532 \\ \text { z155 } & z & 155 & 18357.85 & 18357.85 & -0.00174 & -0.09456 \\ \text { y-155 } & y- & 155 & 18372.87 & 18372.91 & 0.046894 & 2.552353 \\ \text { x+155 } & x+ & 155 & 18400.86 & 18400.91 & 0.053688 & 2.917688 \\ \text { y-158 } & y- & 158 & 18585.98 & 18586.02 & 0.040789 & 2.194613 \\ \text { y-161 } & y- & 161 & 18813.1 & 18813.15 & 0.049795 & 2.646827 \\ \text { z162 } & z & 162 & 18897.16 & 18897.19 & 0.027353 & 1.447466 \\ \text { y164 } & y & 164 & 19125.33 & 19125.43 & 0.097993 & 5.123728 \\ \text { z165 } & z & 165 & 19237.41 & 19237.49 & 0.085715 & 4.455642 \\ \text { x165 } & x & 165 & 19279.41 & 19279.51 & 0.100511 & 5.213387 \\ \text { z167 } & z & 167 & 19529.51 & 19529.7 & 0.187362 & 9.593787 \\ \text { y-169 } & y- & 169 & 19776.61 & 19776.76 & 0.144949 & 7.329315\end{array}$


Table S8C. Summary of UVPD mass spectrum for WT•GDPnP complex: fragment ions incorporating mass shift of + 541.9604 Da (GDPnP)

\begin{tabular}{|c|c|c|c|c|c|c|}
\hline & $\underline{\text { Ion }}$ & & Theoretical & Experimental & & $\mathrm{ppm}$ \\
\hline Ion & Type & Residue & Mass & Mass & $\Delta$ mass & error \\
\hline$c 22$ & c & 22 & 2690.158 & 2690.165 & 0.007571 & 2.814333 \\
\hline$a+124$ & $a+$ & 124 & 14496.84 & 14496.97 & 0.122471 & 8.448112 \\
\hline c124 & c & 124 & 14540.86 & 14540.99 & 0.13618 & 9.365335 \\
\hline c127 & c & 127 & 14856 & 14856.04 & 0.040758 & 2.743538 \\
\hline a127 & $a$ & 127 & 14810.98 & 14811.06 & 0.079063 & 5.338134 \\
\hline$a+131$ & $a+$ & 131 & 15240.23 & 15240.29 & 0.068747 & 4.510889 \\
\hline c134 & c & 134 & 15640.41 & 15640.51 & 0.105579 & 6.750399 \\
\hline a134 & $\mathrm{a}$ & 134 & 15595.39 & 15595.53 & 0.143809 & 9.221252 \\
\hline c135 & c & 135 & 15711.45 & 15711.55 & 0.101109 & 6.435372 \\
\hline b138 & $b$ & 138 & 16100.62 & 16100.75 & 0.131378 & 8.159812 \\
\hline$a+139$ & $a+$ & 139 & 16130.65 & 16130.78 & 0.127526 & 7.905817 \\
\hline c141 & c & 141 & 16384.8 & 16384.81 & 0.008818 & 0.538182 \\
\hline a141 & a & 141 & 16339.78 & 16339.9 & 0.121892 & 7.459831 \\
\hline a145 & a & 145 & 16830.02 & 16830.03 & 0.009434 & 0.560546 \\
\hline$a+153$ & $a+$ & 153 & 17658.49 & 17658.46 & -0.027 & -1.52901 \\
\hline b154 & $b$ & 154 & 17800.51 & 17800.54 & 0.037095 & 2.08393 \\
\hline b155 & $b$ & 155 & 17915.53 & 17915.57 & 0.034522 & 1.926931 \\
\hline$a+164$ & $a+$ & 164 & 18981.14 & 18981.33 & 0.189059 & 9.960358 \\
\hline c165 & c & 165 & 19181.26 & 19181.39 & 0.133397 & 6.95455 \\
\hline$x+8$ & $x+$ & 8 & 1635.572 & 1635.581 & 0.008785 & 5.371189 \\
\hline$y-9$ & $y^{-}$ & 9 & 1763.678 & 1763.676 & -0.00207 & -1.17537 \\
\hline$x+20$ & $x+$ & 20 & 3000.245 & 3000.254 & 0.009075 & 3.024743 \\
\hline$y-20$ & $y^{-}$ & 20 & 2972.25 & 2972.267 & 0.017173 & 5.777789 \\
\hline$x+32$ & $x+$ & 32 & 4300.959 & 4301 & 0.041455 & 9.638542 \\
\hline$x 68$ & $x$ & 68 & 8314.976 & 8314.963 & -0.01317 & -1.58353 \\
\hline$x+71$ & $x+$ & 71 & 8685.222 & 8685.285 & 0.063037 & 7.257957 \\
\hline$y-75$ & $y^{-}$ & 75 & 9242.493 & 9242.547 & 0.053975 & 5.839878 \\
\hline$y-76$ & $y-$ & 76 & 9379.552 & 9379.636 & 0.084703 & 9.030606 \\
\hline$y-78$ & $y-$ & 78 & 9607.663 & 9607.732 & 0.069926 & 7.278152 \\
\hline y118 & $y$ & 118 & 14165.8 & 14165.92 & 0.123685 & 8.73124 \\
\hline$x+121$ & $x+$ & 121 & 14525.89 & 14525.99 & 0.106715 & 7.346538 \\
\hline y122 & $y$ & 122 & 14555.92 & 14555.97 & 0.050372 & 3.460585 \\
\hline$x 122$ & $x$ & 122 & 14581.9 & 14581.96 & 0.062318 & 4.273654 \\
\hline$x+124$ & $x+$ & 124 & 14811.02 & 14811.06 & 0.040173 & 2.71237 \\
\hline$y-126$ & $y^{-}$ & 126 & 14981.16 & 14981.23 & 0.065135 & 4.347796 \\
\hline$x 127$ & $x$ & 127 & 15136.21 & 15136.24 & 0.038144 & 2.52005 \\
\hline y127 & $y$ & 127 & 15110.23 & 15110.27 & 0.043288 & 2.864815 \\
\hline z127 & z & 127 & 15094.21 & 15094.26 & 0.054973 & 3.641993 \\
\hline
\end{tabular}




\begin{tabular}{|c|c|c|c|c|c|c|}
\hline x129 & $x$ & 129 & 15420.4 & 15420.44 & 0.035024 & 2.271277 \\
\hline$x+130$ & $x+$ & 130 & 15584.47 & 15584.42 & -0.05154 & -3.30733 \\
\hline x131 & $x$ & 131 & 15670.5 & 15670.53 & 0.036265 & 2.314221 \\
\hline$x+132$ & $x+$ & 132 & 15786.53 & 15786.59 & 0.05985 & 3.791204 \\
\hline z136 & $z$ & 136 & 16183.75 & 16183.79 & 0.036972 & 2.284513 \\
\hline y138 & y & 138 & 16477.86 & 16478.02 & 0.159315 & 9.668426 \\
\hline z139 & z & 139 & 16590.89 & 16590.95 & 0.05883 & 3.545923 \\
\hline z140 & $z$ & 140 & 16705.91 & 16706.04 & 0.129058 & 7.725289 \\
\hline x140 & $x$ & 140 & 16747.91 & 16748.06 & 0.145676 & 8.698159 \\
\hline$y-141$ & $y-$ & 141 & 16819.99 & 16820.09 & 0.097202 & 5.778958 \\
\hline z142 & $z$ & 142 & 16952.05 & 16952.18 & 0.127003 & 7.491896 \\
\hline x142 & $x$ & 142 & 16994.05 & 16994.19 & 0.143622 & 8.451312 \\
\hline y143 & $y$ & 143 & 17105.13 & 17105.16 & 0.03175 & 1.856169 \\
\hline y146 & y & 146 & 17460.31 & 17460.38 & 0.067299 & 3.854398 \\
\hline$x+147$ & $x+$ & 147 & 17600.38 & 17600.43 & 0.042656 & 2.423582 \\
\hline$y-149$ & $y-$ & 149 & 17813.53 & 17813.63 & 0.093125 & 5.227769 \\
\hline y150 & $y$ & 150 & 17915.59 & 17915.57 & -0.02097 & -1.17066 \\
\hline z150 & $z$ & 150 & 17899.57 & 17899.59 & 0.017615 & 0.984102 \\
\hline z154 & z & 154 & 18298.82 & 18298.85 & 0.034347 & 1.877007 \\
\hline x154 & $x$ & 154 & 18340.82 & 18340.89 & 0.072206 & 3.936902 \\
\hline$y-157$ & $y^{-}$ & 157 & 18526.94 & 18526.99 & 0.04611 & 2.48881 \\
\hline y159 & $y$ & 159 & 18656.01 & 18656.04 & 0.033671 & 1.804834 \\
\hline$y-160$ & $y-$ & 160 & 18712.02 & 18712.06 & 0.043961 & 2.349347 \\
\hline$x+160$ & $x+$ & 160 & 18740.01 & 18740.08 & 0.061009 & 3.255545 \\
\hline z161 & z & 161 & 18796.08 & 18796.08 & 0.001872 & 0.099595 \\
\hline x161 & $x$ & 161 & 18838.07 & 18838.14 & 0.067075 & 3.560608 \\
\hline y162 & $y$ & 162 & 18911.16 & 18911.19 & 0.022156 & 1.171583 \\
\hline y165 & y & 165 & 19251.41 & 19251.52 & 0.108477 & 5.634756 \\
\hline$x+167$ & $x+$ & 167 & 19570.5 & 19570.53 & 0.024792 & 1.266803 \\
\hline
\end{tabular}


Table S8D. Summary of UVPD mass spectrum for WT•GDPnP complex: fragment ions incorporating mass shift of +542.9526 Da (GDPnP)

\begin{tabular}{|c|c|c|c|c|c|c|}
\hline & Ion & & Theoretical & Experimental & & $\mathrm{ppm}$ \\
\hline Ion & Type & Residue & Mass & Mass & $\Delta$ mass & error \\
\hline c1 & c & 1 & 617.0004 & 617 & -0.00036 & -0.58347 \\
\hline$a+10$ & $a+$ & 10 & 1635.565 & 1635.581 & 0.01567 & 9.580766 \\
\hline$a+15$ & $a+$ & 15 & 1976.735 & 1976.732 & -0.00299 & -1.51008 \\
\hline a124 & $a$ & 124 & 14496.83 & 14496.97 & 0.138096 & 9.525945 \\
\hline c126 & c & 126 & 14741.97 & 14742.02 & 0.051239 & 3.475724 \\
\hline a131 & $\mathrm{a}$ & 131 & 15240.21 & 15240.29 & 0.084372 & 5.536144 \\
\hline a139 & $\mathrm{a}$ & 139 & 16130.63 & 16130.78 & 0.143151 & 8.87448 \\
\hline c143 & c & 143 & 16645.95 & 16646.02 & 0.078055 & 4.68913 \\
\hline b147 & $b$ & 147 & 17017.08 & 17017.1 & 0.026488 & 1.556554 \\
\hline$a+150$ & $a+$ & 150 & 17375.33 & 17375.34 & 0.000296 & 0.017034 \\
\hline c153 & c & 153 & 17703.5 & 17703.47 & -0.0299 & -1.68916 \\
\hline a153 & $a$ & 153 & 17658.48 & 17658.46 & -0.01137 & -0.64417 \\
\hline$a+160$ & $a+$ & 160 & 18484.84 & 18484.99 & 0.156115 & 8.445569 \\
\hline$a+162$ & $a+$ & 162 & 18740.01 & 18740.08 & 0.067894 & 3.622942 \\
\hline$a+163$ & $a+$ & 163 & 18869.05 & 18869.15 & 0.100161 & 5.308215 \\
\hline b166 & $b$ & 166 & 19293.32 & 19293.4 & 0.08542 & 4.42744 \\
\hline$x+7$ & $x+$ & 7 & 1507.522 & 1507.51 & -0.01163 & -7.71202 \\
\hline y9 & $y$ & 9 & 1765.678 & 1765.674 & -0.00405 & -2.2943 \\
\hline z9 & $z$ & 9 & 1749.66 & 1749.656 & -0.00372 & -2.12556 \\
\hline$x 20$ & $x$ & 20 & 3000.229 & 3000.254 & 0.0247 & 8.232705 \\
\hline$x 71$ & $x$ & 71 & 8685.206 & 8685.285 & 0.078662 & 9.05701 \\
\hline$z 72$ & $z$ & 72 & 8772.251 & 8772.269 & 0.018355 & 2.092393 \\
\hline z73 & z & 73 & 8928.352 & 8928.377 & 0.025033 & 2.803765 \\
\hline$x+77$ & $x+$ & 77 & 9521.623 & 9521.698 & 0.075697 & 7.950007 \\
\hline$y-118$ & $y-$ & 118 & 14165.78 & 14165.92 & 0.13931 & 9.834262 \\
\hline y120 & $y$ & 120 & 14370.85 & 14370.9 & 0.052406 & 3.646688 \\
\hline x121 & $x$ & 121 & 14525.87 & 14525.99 & 0.12234 & 8.422215 \\
\hline$y-122$ & $y-$ & 122 & 14555.9 & 14555.97 & 0.065997 & 4.534038 \\
\hline z122 & z & 122 & 14540.89 & 14540.99 & 0.10013 & 6.886097 \\
\hline y123 & $y$ & 123 & 14671.94 & 14672 & 0.05962 & 4.063539 \\
\hline x124 & $x$ & 124 & 14811 & 14811.06 & 0.055798 & 3.767334 \\
\hline$x+126$ & $x+$ & 126 & 15010.15 & 15010.23 & 0.081074 & 5.401277 \\
\hline$y-127$ & $y-$ & 127 & 15110.21 & 15110.27 & 0.058913 & 3.898889 \\
\hline$x+128$ & $x+$ & 128 & 15266.3 & 15266.34 & 0.034712 & 2.273764 \\
\hline x130 & $x$ & 130 & 15584.46 & 15584.42 & -0.03592 & -2.30473 \\
\hline y132 & $y$ & 132 & 15760.54 & 15760.57 & 0.036813 & 2.335771 \\
\hline x132 & $x$ & 132 & 15786.52 & 15786.59 & 0.075475 & 4.780979 \\
\hline$x+135$ & $x+$ & 135 & 16130.7 & 16130.78 & 0.079111 & 4.904373 \\
\hline
\end{tabular}




$\begin{array}{llrrrrr}\text { y136 } & y & 136 & 16200.76 & 16200.79 & 0.02931 & 1.809174 \\ \text { y137 } & y & 137 & 16315.79 & 16315.89 & 0.096926 & 5.940625 \\ \text { x+138 } & x+ & 138 & 16505.84 & 16505.93 & 0.083786 & 5.07614 \\ \text { x+141 } & x+ & 141 & 16848.98 & 16849.04 & 0.056245 & 3.338182 \\ \text { y142 } & y & 142 & 16969.06 & 16969.07 & 0.004222 & 0.248806 \\ \text { y-143 } & y- & 143 & 17105.11 & 17105.16 & 0.047375 & 2.769642 \\ \text { y144 } & y & 144 & 17220.16 & 17220.21 & 0.049843 & 2.894456 \\ \text { x+145 } & x+ & 145 & 17375.21 & 17375.34 & 0.126731 & 7.293781 \\ \text { y-146 } & y- & 146 & 17460.3 & 17460.38 & 0.082924 & 4.749291 \\ \text { x147 } & x & 147 & 17600.37 & 17600.43 & 0.058281 & 3.311351 \\ \text { x+148 } & x+ & 148 & 17729.44 & 17729.5 & 0.068488 & 3.862953 \\ \text { y149 } & y & 149 & 17815.53 & 17815.57 & 0.033822 & 1.898456 \\ \text { x+149 } & x+ & 149 & 17842.52 & 17842.58 & 0.058939 & 3.303287 \\ \text { y-150 } & y- & 150 & 17915.57 & 17915.57 & -0.00535 & -0.29851 \\ \text { y155 } & y & 155 & 18372.85 & 18372.91 & 0.062469 & 3.400071 \\ \text { y158 } & y & 158 & 18585.96 & 18586.02 & 0.056364 & 3.032612 \\ y-159 & y- & 159 & 18655.99 & 18656.04 & 0.049296 & 2.64237 \\ x 160 & x & 160 & 18740 & 18740.08 & 0.076634 & 4.089328 \\ y 161 & y & 161 & 18813.09 & 18813.15 & 0.06537 & 3.474709 \\ y-162 & y- & 162 & 18911.15 & 18911.19 & 0.037781 & 1.997818 \\ x+165 & x+ & 165 & 19279.39 & 19279.51 & 0.116086 & 6.021247 \\ y-165 & y- & 165 & 19251.4 & 19251.52 & 0.124102 & 6.446391 \\ x 167 & x & 167 & 19570.49 & 19570.53 & 0.040417 & 2.065201 \\ \text { y169 } & y & 169 & 19776.6 & 19776.76 & 0.160524 & 8.116866\end{array}$


Supplemental Table 9. Summary of residues for which UVPD is enhanced or suppressed

\begin{tabular}{|c|l|l|}
\hline $\begin{array}{c}\text { K-Ras } \\
\text { Construct }\end{array}$ & $\begin{array}{l}\text { Enhanced UVPD ( >+0.0001 TIC } \\
\text { Abundance Change) }\end{array}$ & $\begin{array}{l}\text { Suppressed UVPD (<-0.0001 TIC } \\
\text { Abundance Change) }\end{array}$ \\
\hline WT & $1,3,5,6,9,15,16,20,28,30-32$, & $\begin{array}{l}2,7,8,11,18,24,73,102,121,122,125,126, \\
138,139,147,155,157,159,160,164\end{array}$ \\
& $44,46,127,141,154$ & $\begin{array}{l}2,11,22,25,73,92,121,122,125,127,128,130, \\
132-136,138,140,141,144,145-148,155,158\end{array}$ \\
\hline G12C & $1,3,15,16,24,26,30-32,41-48,50,167$ & $1,8,12,19,23,28,29,31,33,34,37,41-46$, \\
& & $48,51,53,138$ \\
\hline G12V & $2,3,6,9,10,13,25,162,167$ & $2,16,28$ \\
& & \\
\hline G12S & $1,3,5,6,8-15,23-25,29-33,41-47$, & \\
& $50,140,143,144,148,157,164,167$ &
\end{tabular}

